\title{
Strategies for optimization of Aminoglycoside and Vancomycin therapies : a Pharmacokinetic approach
}

Citation for published version (APA):

van Maarseveen, E. M. (2014). Strategies for optimization of Aminoglycoside and Vancomycin therapies : a Pharmacokinetic approach. [Doctoral Thesis, Maastricht University]. Maastricht University. https://doi.org/10.26481/dis.20141128em

Document status and date:

Published: 01/01/2014

DOI:

10.26481/dis.20141128em

Document Version:

Publisher's PDF, also known as Version of record

\section{Please check the document version of this publication:}

- A submitted manuscript is the version of the article upon submission and before peer-review. There can be important differences between the submitted version and the official published version of record.

People interested in the research are advised to contact the author for the final version of the publication, or visit the DOI to the publisher's website.

- The final author version and the galley proof are versions of the publication after peer review.

- The final published version features the final layout of the paper including the volume, issue and page numbers.

Link to publication

\footnotetext{
General rights rights.

- You may freely distribute the URL identifying the publication in the public portal. please follow below link for the End User Agreement:

www.umlib.nl/taverne-license

Take down policy

If you believe that this document breaches copyright please contact us at:

repository@maastrichtuniversity.nl

providing details and we will investigate your claim.
}

Copyright and moral rights for the publications made accessible in the public portal are retained by the authors and/or other copyright owners and it is a condition of accessing publications that users recognise and abide by the legal requirements associated with these

- Users may download and print one copy of any publication from the public portal for the purpose of private study or research.

- You may not further distribute the material or use it for any profit-making activity or commercial gain

If the publication is distributed under the terms of Article $25 \mathrm{fa}$ of the Dutch Copyright Act, indicated by the "Taverne" license above, 


\section{Strategies for Optimization of Aminoglycoside and Vancomycin Therapies}
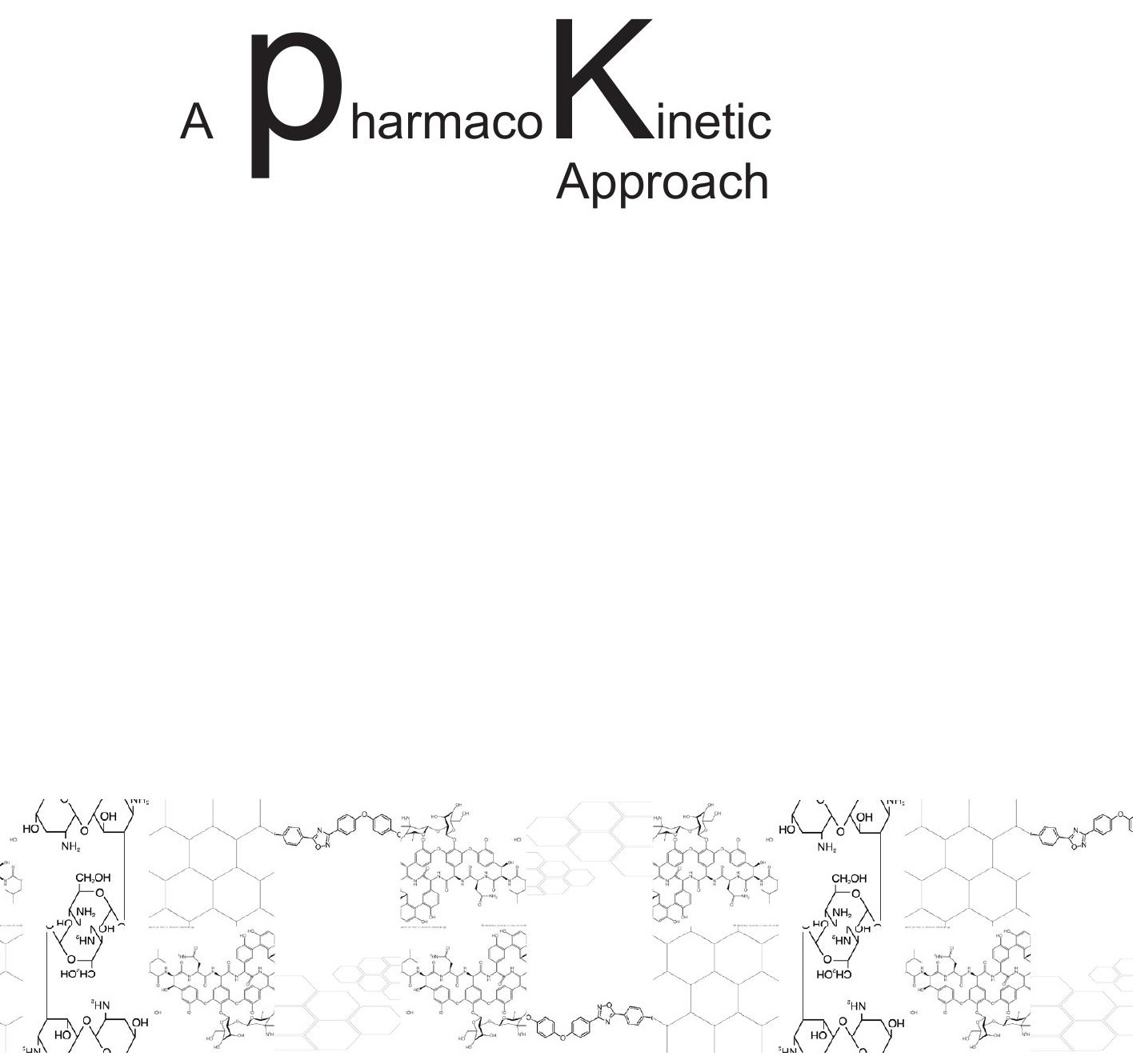
Strategies for Optimization of Aminoglycoside and Vancomycin Therapies

(c) 2014 E.M. van Maarseveen, Utrecht The Netherlands Lay-out and cover design by Marieke Velthuis

Printed by: Gildeprint - The Netherlands

Printed on FSC certified paper

ISBN/EAN 9789461087966 


\title{
Strategies for Optimization of Aminoglycoside and Vancomycin Therapies
}

A Pharmacokinetic Approach

\section{PROEFSCHRIFT}

\author{
ter verkrijging van \\ de graad van doctor aan de Universiteit Maastricht, \\ op gezag van de Rector Magnificus, Prof dr. L.L.G. Soete \\ volgens het besluit van het College van Decanen, \\ in het openbaar te verdedigen \\ op vrijdag 28 november 2014 om 14.00 uur
}

door

Erik Michiel van Maarseveen

Geboren op 17 mei 1979 te Tiel 


\section{Promotores}

Prof. dr. C. Neef

Prof. dr. D. Touw (Universitair Medisch Centrum Groningen)

\section{Copromotor}

Dr. A. van Zanten (Ziekenhuis Gelderse Vallei Ede)

\section{Beoordelingscommissie}

Prof. dr. P. Savelkoul (voorzitter)

Dr. R. Aarnoutse (Universitair Medisch Centrum Nijmegen)

Prof. dr. T. van Gelder (Erasmus Medisch Centrum Rotterdam)

Prof. dr. H. Struijker-Boudier 
Voor mijn vader 



\section{Table of Contents}

$\begin{array}{lll}\text { Chapter } 1 \quad \text { General introduction } & 11\end{array}$

$\begin{array}{lll}\text { Chapter } 1.1 & \text { Aminoglycosides } & 11\end{array}$

Chapter 1.2 Continous and intermittent infusion of vancomcycin are equally effective:

a review of literature

Chapter 2

Chapter 2.1. The effect of the circadian rhythm on the pharmacokinetics of once daily tobramycin in cystic fibrosis patients

Chapter 2.2 Chronopharmacokinetics of once daily dosed aminoglycosides in clinically infected patients

Chapter 2.3. Once daily dosed gentamicin is more nephrotoxic than once daily dosed tobramycin in clinically Infected patients

Chapter 2.4. Extended-interval dosing of gentamicin in neonates targeting trough concentrations under $0.5 \mathrm{mg} / \mathrm{L}$ : how low can we go?

Chapter 3

Chapter 3.1. Vancomycin exposure variability of intermittent versus continuous infusion: a systematic review

Chapter 3.2. Exposure variability and target attainment of vancomycin intermittent and continuous infusion in critically ill patients -ready, aim, target locked? 
Chapter 3.3. Design and prospective validation of a dosing instrument for continuous infusion of vancomycin:

a within-population approach

Chapter 4 Summary, clinical implications and future perspectives

Nederlandse samenvatting

Dankwoord

List of publications

Valorization Paragraph

Curriculum Vitae

203 


\section{Chapter 1}

\section{General introduction}

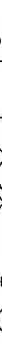





\subsection{Aminoglycosides}

Aminoglycosides are potent bactericidal antibiotics and used to treat severe infections primarily caused by Gram-negative bacteria, staphylococci as well as certain mycobacteria. Most common indications for the parenteral formulation are urinary tract, intra-abdominal and surgical infections, infective endocarditis, (neonatal) sepsis, and pulmonary exacerbation of cystic fibrosis (CF) $(1,2)$. Aminoglycosides are a cornerstone of the current antibiotic armamentarium because of their activity against multidrug-resistant Gram negative pathogens such as Pseudomonas aeruginosa and Acinetobacter spp $(2,3)$. Furthermore, to kill host pathogens combinations of aminoglycosides and beta-lactam antibiotics act synergistically and combining both antibiotic classes lowers the risk of bacterial resistance (4). Although very effective, aminoglycosides have a narrow therapeutic range and can induce both severe nephrotoxicity and ototoxicity $(3,4)$.

\section{Mechanism of action}

Aminoglycosides inhibit the protein synthesis of microorganisms and as a result alter the integrity of the bacterial cell membrane. Aminoglycosides bind to negatively charged residues in the outer membrane of Gram-negative bacteria (5). When the antibiotic diffuses through the outer membrane, low affinity binding of aminoglycosides to an energy-dependent transport system occurs. The transport across the cytosolic membrane is the rate-limiting step and can be blocked by calcium and magnesium ions, hyperosmolarity, low pH and anaerobic conditions. In the cytosol, aminoglycosides bind to $30 \mathrm{~S}$ subunit ribosomes and block the translation process resulting in the disruption of chain elongation of proteins. Aberrant proteins are then inserted into the cell membrane, resulting in altered permeability and further stimulation of transmembrane aminoglycoside transport (6). Finally, disruption of the structure of the cell membrane leads to a leakage of intracellular contents, which precedes cell death (7). 


\section{Efficacy}

The bactericidal activity of aminoglycosides is concentration-dependent (8). Importantly, aminoglycosides have demonstrated a post-antibiotic effect (PAE) against gram-negative bacteria (9). PAE refers to continued suppression of bacterial growth after antibiotic concentrations have dropped below the Minimal Inhibitory Concentration (MIC) (10). This allows a longer dose interval than predicted based on the MIC alone (11). The pharmacokinetic/pharmacodynamic predictors associated with efficacy are ratio of peak plasma drug concentration over the $\mathrm{MIC}$ of the causative pathogen $(\mathrm{Cmax} / \mathrm{MIC})$ and ratio of the area under the concentration-time curve (AUC) over MIC (AUC/MIC) (12). Target Cmax and AUC values of respectively $8-10 \mathrm{mg} / \mathrm{L}$ and $74-100 \mathrm{mg} . \mathrm{h} . \mathrm{L}-1$ are recommended $(1,2)$. For microorganisms with an MIC of $2 \mathrm{mg} / \mathrm{L}$, a Cmax of at least $20 \mathrm{mg} / \mathrm{L}$ is recommended (1).

\section{Toxicity}

Nephro- and ototoxicity are the most frequently observed side effects of aminoglycosides (13). Aminoglycosides induce nephrotoxicity in an estimated $0-35 \%$ of treated patients based on reports from literature (14). Albeit aminoglycoside-associated nephrotoxicity is generally defined by an increase in serum creatinine by more than $0.5 \mathrm{mg} / \mathrm{dL}$ (44 $\mathrm{nmol} / \mathrm{mL}$ ) or increase more than $50 \%$ in serum creatinine from baseline, clinical studies report divergent definitions of nephrotoxicity which may partially explain this broad range of reported incidences of nephrotoxicity. Without intervention aminoglycoside-induced nephrotoxicity eventually manifests as nonoliguric renal failure (15). It is characterized by a decrease in glomerular filtration rate, tubular necrosis and as a consequence a rise in serum creatinine $(14,15)$.

After glomerular filtration, approximately $15 \%$ of the amount of filtered aminoglycoside is reabsorbed into the renal tissue (16) and retained in the epithelial lining cells of the proximal tubules. Aminoglycosides enter the endosomal compartment through endocytosis via the megalin-cubilin complex (17). When the concentrations of aminoglycosides exceed an undetermined threshold, the endosomal membrane destabilizes and aminoglycosides are released into the cytosol $(17,18)$. Aminoglycosides bind to phospholipids membranes to cause phospholipidosis (17). Cytosolic aminoglycosides and phospholipidosis promote cell apoptosis 
$(15,17,18)$. Aminoglycoside-associated nephrotoxicity is directly correlated to accumulation of the drug in the renal proximal tubules. This accumulation depends on several factors such as the number of amino groups and the distribution of these groups within the molecule and thus can differ within the aminoglycoside drug class. Aminoglycosides with a high number of positively charged amino groups at physiological $\mathrm{pH}$ are more likely to cause nephrotoxicity, perhaps due to enhanced interactions with anionic membrane phospholipids and megalin $(15,17$, 18). Drug steric hindrance can also influence the affinity of the drug to the receptor binding domain. These factors are responsible for the cationic structure of the molecule and determine the potency of the binding affinity of aminoglycosides to the brush border's megalin receptor (15, $17,18)$. Figure 1 displays potential cellular mechanisms of toxicity of the aminoglycoside gentamicin.

Ototoxic side effects of aminoglycosides usually occur within days or weeks after initiation of systemic application (19). Aminoglycosides can induce irreversible damage both to the auditory and vestibular organs. Gentamicin and tobramycin are predominantly vestibulotoxic, while neomycin, kanamycin and amikacin are mainly cochleotoxic (20). The prevalence of vestibulotoxicity and cochleotoxicity are $15 \%$ and $2-25 \%$, respectively $(21,22)$. Symptoms of cochleotoxicity are hearing loss and/or tinnitus, while those of vestibulotoxicity are dizziness and light-headedness. Cochleotoxicity initially affects the high auditive frequencies and subsequently may extend towards the lower frequencies (19). Threshold monitoring by pure-tone audiograms in the conventional $<8 \mathrm{kHz}$ and high-frequency regions $8-16$ or $20 \mathrm{kHz}$ is recommended (23). Aminoglycoside ototoxicity is characterized by degeneration of hair cells located in the organ of Corti, particularly in the basal turn which is required to sense high-frequency sounds. In rare cases aminoglycosides can also cause damage to nervus VIII (nervus vestibulocochlearis) resulting in complete deafness (24). 
Figure 1. Potential cellular mechanism of gentacin toxicity (printed with permission and at the courtesy of Dr. Andrew Prayle, Nottingham, UK)

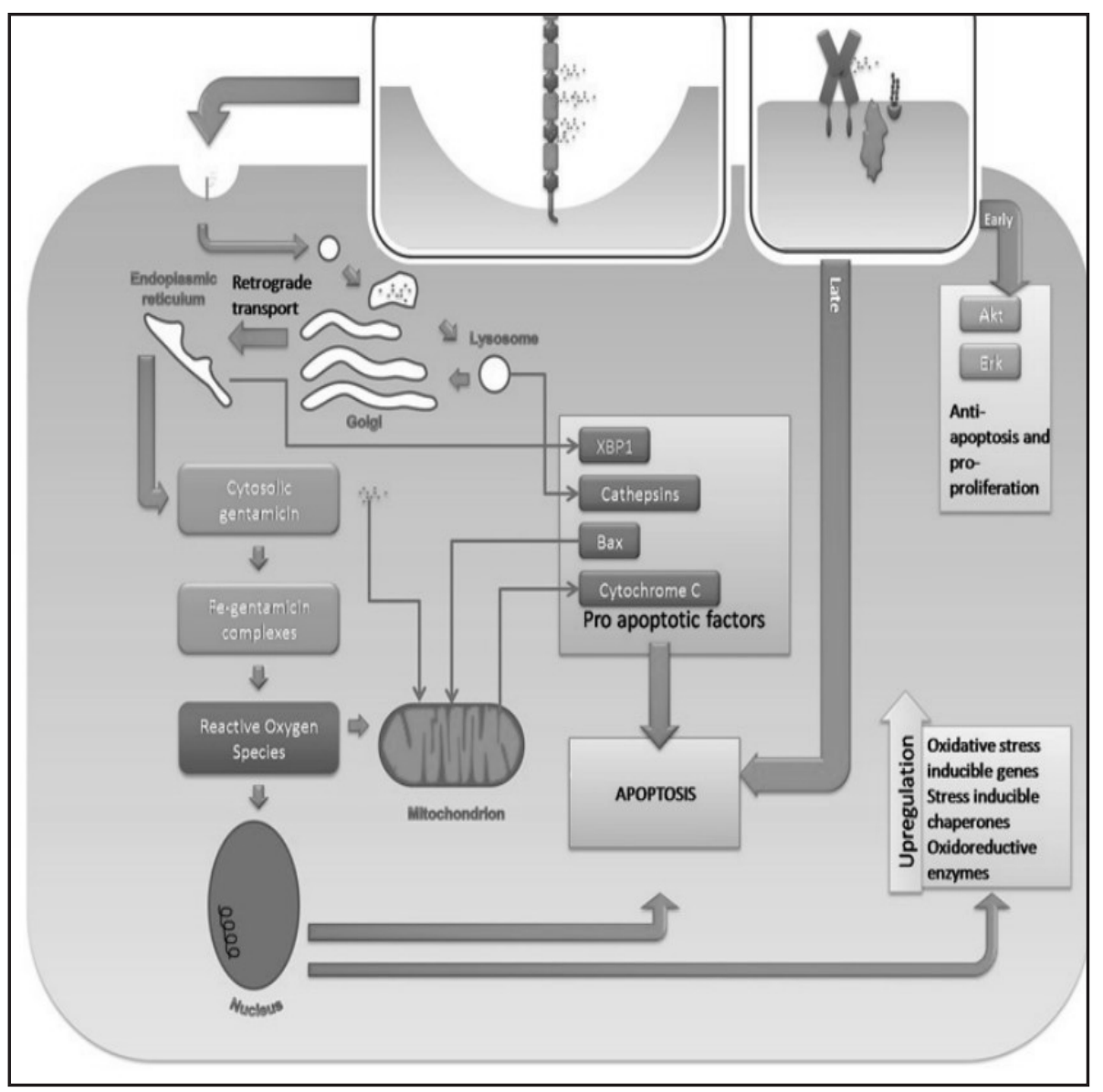

This diagram summarizes the major mechanisms of nephrotoxicity from the literature, mainly from in vitro research and as such has to be interpreted as a model. Gentamicin enters the tubular cell via the multiligand receptor megalin and subsequently uptake is via a clatharin-coated pit. Gentamicin retrogradely passes through the lysosomes, Golgi and endoplasmic reticulum into the cytoplasm where it forms complexes with iron which catalyse the formation of reactive oxygen species (ROS). In the lysosomes, gentamicin causes release of the pro-apoptotic cathepsins and, in the endoplasmic reticulum, gentamicin causes release of the pro-apoptotic stress protein von Hippel-Lindau binding protein 1 (VBP1). Cytosolic gentamicin either directly or indirectly (via ROS) causes the release of cytochrome $C$ from the mitochondria, a key step in apoptosis. ROS cause an increase in the gene expression of oxidative stress-inducible genes, stress-inducible chaperones and oxido-reductive enzymes. Gentamicin may have intracellular effects without entry to the cell. It can cause a rapid increase in intracellular $\mathrm{Ca} 2+$ and an early increase in the anti-apoptosis and proliferative signals Akt and Erk; this may be mediated by the membrane $\mathrm{Ca} 2+$ receptor $\mathrm{CaR}$. 


\section{Aminoglycoside pharmacology and monitoring in highly exposed populations}

Patients suffering from cystic fibrosis (CF), severe infections in the intensive care unit (ICU) or neonatal sepsis caused by Gram-negative bacilli are frequently treated with aminoglycosides. In the following paragraphs aminoglycoside therapy in these specific patient populations is addressed.

\section{Cystic fibrosis patients}

During childhood CF lungs are predominately colonized with Staphylococcus aureus and/or Haemophilus influenzae, and at adolescent and adult ages with Pseudomonas aeruginosa as the disease progresses (25). Intravenous aminoglycosides in combination with beta-lactam antibiotics are commonly used to treat moderate to severe acute pulmonary exacerbations of CF caused by the latter pathogen $(26,27)$. Aminoglycoside pharmacokinetics in CF patients show specific differences from other aminoglycoside treated populations. Malnourished CF patients due to the reduced adipose tissue mass, will have an increase in lean tissue per $\mathrm{kg}$ bodyweight $(28,29)$. As the volume of distribution is related to the lean body mass (LBM), a higher dose is required to attain predefined Cmax/MIC targets. Additionally, AUC/MIC ratios are affected by a relatively high renal clearance in CF patients compared to other clinically infected patient populations and is associated with their relatively young age (12). Once-daily aminoglycoside treatment confers equivalent efficacy to a multiple-daily dosing, however has shown to be less nephrotoxic in children with CF (30-32). The current standard duration for intravenous aminoglycoside therapy is 14 days or longer in case of severe exacerbations or incomplete recovery (27). The present recommended intravenous tobramycin dose for adult CF patients is 10 $\mathrm{mg} / \mathrm{kg} /$ day and $30-35 \mathrm{mg} / \mathrm{kg} /$ day for amikacin (33). Since CF patients usually are exposed to multiple courses of prolonged aminoglycoside exposure during their life, they are at high risk of developing aminoglycoside side effects such as nephrotoxicity (34). Not only monitoring of the renal function, but also therapeutic drug monitoring (TDM) is essential in these patients to optimize the efficacy and limit acute and cumulative toxicity. 
The Cmax targets of tobramycin and amikacin are 20-40 $\mathrm{mg} / \mathrm{L}$ and $80-120 \mathrm{mg} / \mathrm{L}$, respectively, with a Cmin target of $<1 \mathrm{mg} / \mathrm{L}$ for both aminoglycosides (33).

\section{Critically ill patients}

Aminoglycosides play an important role in the treatment of critically ill patients with severe sepsis, hospital-acquired pneumonia, ventilator-associated pneumonia, health care-associated pneumonia, urinary tract infections, endocarditis, bacterial meningitis and febrile neutropenia (35). In critically ill patients a large variability in aminoglycoside distribution and clearance due to pathophysiological changes and concomitant therapies compromises the attainment of predefined exposure targets. For instance, fluid resuscitation and total parenteral nutrition expand the extracellular fluid content and dilute serum and extracellular aminoglycoside concentrations. Meanwhile, patients with renal dysfunction and decreased cardiac output have a decreased clearance and therefore are prone to develop aminoglycoside-associated nephrotoxicity $(36,37)$. Achievement of target peak serum concentration is related to the volume of distribution. Thus, dose modification of aminoglycosides will be required more often in populations with high inter- and intra-patient variability in volume of distribution and/or clearance (35). Several nomograms recommend that the dosages of aminoglycosides should be higher compared to other adult non-CF populations; gentamicin or tobramycin $7 \mathrm{mg} / \mathrm{kg}$ and amikacin $15 \mathrm{mg} / \mathrm{kg}$.

Although these dosing nomograms produce good results in clinical practice, many ICU patients do not achieve the target peak or trough serum concentration independent of the selection of a particular 'a priori' dosing algorithm (38-40). Hence, TDM of aminoglycosides is essential in critically ill patients to guarantee efficacy and prevent toxicity in this vulnerable population.

\section{Neonates}

Neonatal early onset infections are usually of maternal-foetal (perinatal) origin and typically occur in the first 5 days of life caused by contaminated amniotic fluid or blood. The predominant pathogens in term neonates include group B streptococci, Escherichia coli, enterococci and Listeria monocytogenes. In preterm neonates, group B streptococci, Escherichia coli, Haemophilus influenzae and Gram-negative bacilli 
are predominant (41). Late-onset infections, on the other hand, occur in the first month of life, particularly from contaminated hands or venous access. Coagulase-negative staphylococci are the main pathogens, especially Staphylococcus epidermis. Pseudomonas spp, Klebsiella spp and Serratia spp are also common pathogens. Neonates demonstrate aberrant pharmacokinetics compared to adult patients. First, neonates have a relatively low fat tissue mass compared to adults. Water comprises about $60-70 \%$ of a neonate's body weight, which affects the volume of distribution of aminoglycosides (42). Therefore, a higher dose of aminoglycoside per kilogram of bodyweight is needed to achieve the target drug peak concentration (43). Furthermore, renal clearance of aminoglycosides is correlated to gestational age and accelerates directly postpartum. Because of these developmental influences, there is great inter- and intra-individual variability in the volume of distribution and clearance of aminoglycosides in neonates, (44). Currently, a minimum drug plasma concentration of $<1-2 \mathrm{mg} / \mathrm{L}$ is targeted to prevent accumulation of aminoglycosides, which may lead to nephrotoxicity and ototoxicity (45). Gentamicin is the most widely used aminoglycoside in neonates. Tobramycin and netilmicin are also frequently used. Numerous gentamicin dosing regimens have been suggested for neonates, based on the bodyweight, gestational age (GA) and postnatal age (PNA) (45).

For example, the widely practiced Christchurch protocol, which was designed using data obtained from a neonatal intensive care unit (NICU) population, consists of a complex dosing equation based on birth weight, with a relatively high $\mathrm{mg} / \mathrm{kg}$ dose and extended dosing intervals up to 60 hours for infants with a relatively low body weight. Prospective evaluation in over four hundred neonates showed that the vast majority $(84 \%)$ of the population reaches the predefined peak concentration target of $>10 \mathrm{mg} / \mathrm{L}$ and $77 \%$ reached target trough plasma concentrations $<2 \mathrm{mg} / \mathrm{L}$ (46). Another dosing regimen of gentamicin intended for preterm neonates with a GA $<35$ weeks recommends an initial dose of $5 \mathrm{mg} / \mathrm{kg}$ every 36 hours. Prospective evaluation showed that $56 \%$ of all peak blood concentrations and $79 \%$ of all trough concentrations were within the target values of $8-10 \mathrm{mg} / \mathrm{L}$ for peak concentration and $<1$ $\mathrm{mg} / \mathrm{L}$ for trough concentration $(47,48)$. A third proposed dosing scheme of tobramycin recommends $4 \mathrm{mg} / \mathrm{kg}$ every 48 hours in neonates with $\mathrm{GA}<32$ weeks, $4 \mathrm{mg} / \mathrm{kg}$ every 36 hours in neonates with GA between 
32 and 37 weeks and $4 \mathrm{mg} / \mathrm{kg}$ every 24 hours in neonates with $\mathrm{GA}>37$ weeks resulting in $>90 \%$ of neonates reaching target blood concentrations of $5-10 \mathrm{mg} / \mathrm{L}$ for peak concentrations and $<2 \mathrm{mg} / \mathrm{L}$ for trough concentrations $(49,50)$. These three examples illustrate that 'a priori' dosing nomograms of aminoglycosides in neonates are very diverse. This large divergence can be explained by a high inter-population variation in pharmacokinetics in neonates and the predefined target exposure.

A 2006 Cochrane Collaboration review of once-daily versus several times a day dosing of gentamicin in neonates found that both methods demonstrated adequate clearance of sepsis, but once-daily dosing was associated with fewer failures to achieve appropriate peak (aiming for $8-10 \mathrm{mg} / \mathrm{L}$ ) and trough (aiming for $<1$ or $2 \mathrm{mg} / \mathrm{L}$ ) concentrations (51). Ototoxicity and nephrotoxicity were not seen with either regimen, although probably they were not investigated in depth. Once-a-day and extended-interval dosing of gentamicin in neonates is now a widely accepted practice $(45,51)$. As the serum creatinine concentration is a poor surrogate for glomerular function in neonates, the assessment of kidney function remains challenging. Importantly, by measurement of sensitive biomarkers clinical studies have shown tubular damage can occur in more than $50 \%$ of neonates and glomerular damage in $10 \%$ of neonates. However, the clinical relevance of these findings is currently unknown. And albeit serious hearing loss has been associated with aminoglycoside-related toxicity (52), causality has not been proven to date in neonates (53).Irrespective of the selected dosing nomogram, monitoring of serum concentrations of aminoglycosides is widely recommended and adopted to optimize the antibiotic therapy in individual neonates (45).

\section{In conclusion}

Aminoglycosides are potent antimicrobial agents. Yet, their clinical use poses serious risks of side effects. Patient populations that show aberrant aminoglycoside pharmacokinetics and/or are exposed to high dosages are potentially susceptible to aminoglycosides-related toxicity. Moreover, therapeutic drug monitoring is recommended to optimize aminoglycoside therapy in individual patients. Finally, biomarker monitoring such as monitoring serum creatinine plasma levels may reflect 
the condition of the kidneys and may help identify patients at risk of developing aminoglycoside-associated nephrotoxicity. 


\title{
Chapter 1.2.
}

Continuous and intermittent infusion of vancomycin are equally effective: a review of the literature.

\author{
Erik van Maarseveen \\ Annemien Bouma \\ Daniel Touw \\ Cees Neef \\ Arthur van Zanten
}

Adapted from Nederlands Tijdschrift voor Geneeskunde. 155(42); A2667

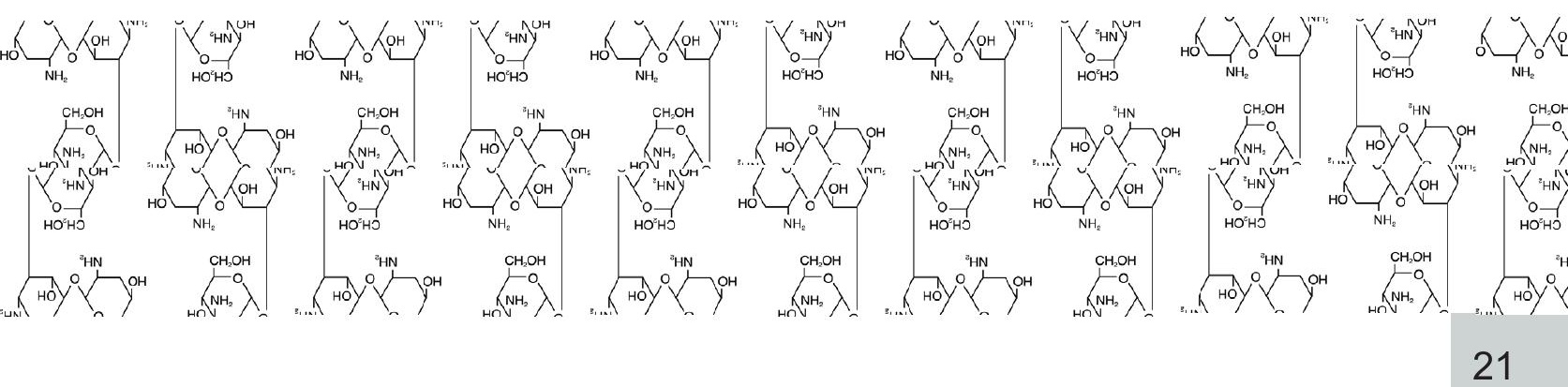




\subsection{Vancomycin}

Vancomycin is a glycopeptide antibiotic used in (suspected or proven) bacteremia, peritonitis or osteomyelitis with gram-positive micro-organisms. The compound was industrially produced by fermentation and given the generic name vancomycin, derived from the term "vanquish." Originally, vancomycin was indicated to treat serious infections caused by penicillin-resistant Gram-positive bacteria. Vancomycin acts by abrogating proper synthesis of the cell wall in Gram-positive bacteria. Due to the different mechanisms by which Gram-negative bacteria produce their cell walls and the various factors related to entering the outer membrane of these pathogens, vancomycin is not active against Gram-negative bacteria (54). Since vancomycin is poorly absorbed after oral intake, it is generally administered through intravenous infusion. The product label states a daily dose of $2000 \mathrm{mg}$, to be infused as 500 $\mathrm{mg}$ every 6 hours or as $1000 \mathrm{mg}$ every 12 hours (55). As there is no gastrointestinal uptake of vancomycin it is administered by intravenous infusion. The initial product label states a daily dose of $2000 \mathrm{mg}$, to be infused as $500 \mathrm{mg}$ every 6 hours or as $1000 \mathrm{mg}$ every 12 hours (52), which was not founded on pharmacodynamic data but was chosen arbitrarily (56). Vancomycin is recommended to be slowly administered in a dilute solution, over at least 60 minutes (maximum infusion rate of $10 \mathrm{mg} /$ minute for doses $>500 \mathrm{mg}$ ) because of the high incidence of pain during infusion, the emergence of thrombophlebitis and to avoid infusion reactions known as the red man syndrome. This syndrome is characterized by flushing or an erythematous rash of face, neck, and torso and usually appears within minutes or hours after the commencement or the completion of a vancomycin infusion.

Antimicrobial killing of vancomycin is time and/or AUC dependent and requires a minimal inhibitory concentration (MIC) of vancomycin at the site of infection $(57,58)$. Although resistance against vancomycin and other glycopeptides is emerging worldwide among methicillin-resistant Staphylococcus aureus and vancomycin-intermediate S. aureus (VISA) strains (59), vancomycin still is the antibiotic of choice for the therapy of methicillin-resistant $S$. aureus (MRSA) infections according to recent guidelines from the Infectious Diseases Society of America (IDSA) (60). To preserve the current therapeutic armamentarium for Gram-positive infections, it is of paramount importance that guidelines 
for vancomycin dosing are based on patient outcomes and on (in vitro) pharmacokinetic/ pharmacodynamic (PK/PD) data to attain maximum efficacy with minimal toxicity. Moreover, this strategy may also prevent the emerge of resistance. Although there is in vitro evidence showing that the time above the MIC and AUC/MIC-values are the most important parameters associated with efficacy, most human studies do not show a clear PK/PD relationship (58). To date, only Moise-Broder et al. found a significant in vivo PK/PD efficacy relation. They showed that for intermittent infusion of vancomycin the AUC_24h/MIC-ratio of $<350$ was associated with a lower eradication rate and higher mortality $(P=$ 0.005 ) in patients with methicillin-resistant $S$. aureus pneumonia (57). Data from recent American studies suggest a reduced susceptibility in case of (suspected) methicillin-resistant Staphylococcus aureus (MRSA) infections to vancomycin urging for higher dosages $(55,61)$. Susceptibility to vancomycin is predicted by determining the in vitro minimum inhibitory concentration. The MIC is the concentration of vancomycin that is needed to inhibit the growth of the strain of bacteria in vitro. The Clinical and Laboratory Standards Institution (CLSI) determines breakpoints for susceptibility and resistance to vancomycin. In 2006, the S. aureus MIC breakpoints for vancomycin were lowered (from $\leq 4 \mu \mathrm{g} / \mathrm{mL}$ to $\leq 2 \mu \mathrm{g} / \mathrm{mL}$ for "susceptible," from 8-16 $\mu \mathrm{g} / \mathrm{mL}$ to $4-8$ $\mu \mathrm{g} / \mathrm{mL}$ for "intermediate," and from $\leq 32 \mu \mathrm{g} / \mathrm{mL}$ to $\leq 16 \mu \mathrm{g} / \mathrm{mL}$ for "resistant") to increase detection of heterogeneously resistant isolates of $S$. aureus (Table 1) (62). The latter is a reflection of the increasing amount of data implying that isolates of $\mathrm{S}$. aureus are less likely to respond to vancomycin therapy. With these new breakpoints, $99 \%$ of MRSA remain within the susceptibility range and should respond to vancomycin therapy. However, three meta-analyses on vancomycin treatment of S. aureus infections observed MICs in the upper limit of the susceptibility range (>1-1.5 mg/L) to be associated with an increased rate of treatment failure and mortality (63-65), leaving room for vancomycin treatment optimization. To optimize the pharmacodynamics of vancomycin some authors suggest that the use of higher intermittent dosages could be helpful (66-68). Nevertheless, based on linear pharmacokinetics the attainment of concentrations above MIC remains a challenge with intermittent infusion (Inl) in patients with a rapid clearance. Hence, it seems rational to explore alternative dosing strategies of vancomycin, for example continuous infusion (Col). 
In the following two sections the currently available studies comparing efficacy and toxicity of continuously and intermittently dosed vancomycin will be reviewed and discussed.

Table 1. Susceptibility and resistance breakpoints for vancomycin against $\mathrm{S}$. aureus

\begin{tabular}{lll}
\hline & $\begin{array}{l}\text { Current breakpoints, MIC } \\
(\mathrm{mg} / \mathrm{L})\end{array}$ & $\begin{array}{l}\text { Breakpoints before 2006, MIC } \\
(\mathrm{mg} / \mathrm{L})\end{array}$ \\
\hline Susceptible S. aureus & $\leq 2$ & $\leq 4$ \\
VISA & $4-8$ & $8-16$ \\
VRSA & $\leq 16$ & $\leq 32$ \\
\hline
\end{tabular}

Abbreviations are MIC, minimum inhibitory concentration; VISA, vancomycinintermediate S. aureus; VRSA, vancomycin-resistant S. aureus

Vancomycin efficacy: a comparison of continuous and intermittent infusion

Vancomycin is thought to inhibit bacterial growth in a time-dependent manner and microbial killing activity therefore depends on the time that vancomycin serum concentrations exceed the MIC. However, this time dependency (time $>\mathrm{MIC}$ ) was not found to be associated with clinical outcomes (57). The PK/PD parameter that is assumed to be the best predictor of efficacy is the area under the concentration-time curve over the minimal inhibitory concentration AUC/MIC $(57,69)$. The consensus guidelines by the American Society of Health-System Pharmacists (ASHP), the Infectious Diseases Society of America (IDSA) and the Society of Infectious Diseases Pharmacists (SIDP) recommend an AUC/MIC ratio $\geq 400$ (61). This is supported by Moise-Broder et al., who found that an AUC/MIC ratio $\geq 350$ is associated with an increased rate of successful clinical outcomes in patients with lower respiratory tract infections caused by S. aureus (57). However, confirmative studies to support these findings have not been reported to date.

Since AUCs are not easily obtained in the clinical setting, trough serum concentrations are monitored as a surrogate marker (68). To obtain an AUC/MIC ratio of $\geq 400$ against bacteria with an MIC $\leq 1 \mathrm{mg} / \mathrm{L}$, it is 
a suggested that a trough serum concentration of at least $15 \mathrm{mg} / \mathrm{L}$ is required with vancomycin $\mathrm{Inl}(70)$. The current dosing advice is to administer 15-20 mg per kg bodyweight every 8-12 hours and to monitor of trough serum concentration, which should be $15-20 \mathrm{mg} / \mathrm{L}$ for severe MRSA infections $(68,71)$. Moreover, higher doses are needed to achieve an AUC/MIC of $\geq 400$ for pathogens with an MIC $>1 \mathrm{mg} / \mathrm{L}$ (72). The need to improve clinical outcomes in patients infected with pathogens that have an MIC $>1 \mathrm{mg} / \mathrm{L}$ for vancomycin, has contributed to the recent debate on the preferential mode of administration of vancomycin: Inl or Col. From a theoretical perspective, Col would be preferred based on the assumption that vancomycin inhibits bacterial growth in a time-dependent manner, since Col is assumed to yield more stable vancomycin serum concentrations and maintain serum concentrations that exceed the MIC. Currently, there is no international consensus on continuous dosing schedules of vancomycin. Some dosing nomograms are based on bodyweight $(73,74)$, where other authors propose dosing schedules based on renal function $(75,76)$. Nevertheless, most protocols recommend monitoring of plateau concentrations, that should reach minimum concentrations of at least $15-25 \mathrm{mg} / \mathrm{L}$ corresponding with an AUC will be between 350 and 600 mg.h.L-1. Consequently, an AUC/MIC of $\geq 400$ cannot be obtained with Col for organisms with MIC $\geq 2 \mathrm{mg} / \mathrm{L}$ without exceeding the target 'plateau' level recommendations. Results from clinical studies may indicate that Col is equivalent to $\mathrm{Inl}$ based on both mortality and treatment failure data. A recent meta-analysis comparing Inl to Col, showed that the relative risk (RR) for overall mortality did not differ between treatment groups (RR 1.03; Cl 0.7-1.6; $p=0.9)$ (77). Since the definition of treatment failure was inconsistent in the studies included in the meta-analysis, this outcome measure could not be pooled. Verrall et al. sought to explore treatment failure between InI and Col in a retrospective study including outpatients.

Treatment failure with Inl was found to be equivalent to Col (RR 0.701; Cl 0.432-1.136; $p=0.159$ ) (78). Vuagnat and co-workers conducted a prospective study in patients with osteomyelitis and found no difference in the percentage of patients cured while treated with either Inl or Col (77.8\% versus $94.4 \%$, respectively; $p=0.25)$. However, patients treated with Inl were more likely to terminate treatment due to adverse reactions than those with $\mathrm{Col}(42.9 \%$ versus $8.7 \%$; $p=0.03)(73)$. In a retrospective cohort study by Boffi El Amari et al., high dose (HD; 40 mg/ $\mathrm{kg} /$ day) was compared to standard dose (SD; $20 \mathrm{mg} / \mathrm{kg} /$ day) in patients with osteomyelitis. The HD regimen was either infused continuously or 
intermittently, whereas SD was only administered as Inl. SD-Inl resulted in a higher risk of treatment failure than $\mathrm{HD}$ (Col and Inl) treatment (OR 7.5; $\mathrm{Cl}$ 1.4-38.6). However, HD resulted in more adverse events compared to SD-Inl ( $25 \%$ versus $4.5 \%$; $p=0.007)$. This was mainly due to HD-Inl, since the rate of adverse events in HD-Col did not differ significantly from SD-InI $(p=0.06)$. Subsequently, patients in the HDCol group had the best outcomes (lowest number of treatment failures and adverse events) compared to the Inl regimens (log rank test $p=$ $0.02)(79)$. Currently, the only randomized clinical trial that compares Inl to Col found no difference in treatment failure (19\% versus $21 \%$, respectively). However, this study by Wysocki et al. was underpowered with respect to treatment failure outcome measures (74).

\section{Vancomycin toxicity: a comparison of continuous and intermittent infusion}

Although treatment failure warrants for higher dosing regimens of vancomycin Inl, the use of higher dosages - to acquire a trough level of $15-20 \mathrm{mg} / \mathrm{L}$ or higher - is limited by the risk of nephrotoxicity. Nephrotoxicity occurs in $5-43 \%$ of patients who are treated with vancomycin, and can partially be explained by determinants reported by clinical studies (80). For example, vancomycin is often administered to critically ill patients admitted to the ICU. In those patients the risk of developing nephrotoxicity is generally higher than for general ward patients as critically ill patients have various other risk factors for acute kidney injury (81). Observational cohort studies have shown that during vancomycin Inl treatment serum trough concentrations of $\geq 15 \mathrm{mg} / \mathrm{L}$ are associated with a higher incidence of nephrotoxicity $(66,82-84)$. Data from other studies suggest that a daily dosage of $>4000 \mathrm{mg}$ could increase the risk of nephrotoxicity (85). In a meta-analysis, Van Hal et al. found an increased incidence of nephrotoxicity with an odds ratio (OR) of 2.67 for high trough serum concentrations ( $\geq 15 \mathrm{mg} / \mathrm{L}$ ) compared to low trough serum concentrations $(<15 \mathrm{mg} / \mathrm{L})(p<0.01)(86)$. Lodise et al. confirmed in a retrospective study that nephrotoxicity is associated with higher trough levels $(\mathrm{Cmin}=14.6 \pm 8.3 \mathrm{mg} / \mathrm{L}$ versus $\mathrm{Cmin}=9.6 \pm 5.1$ $\mathrm{mg} / \mathrm{L}$ in patients with or without nephrotoxicity, respectively). No difference in AUC was found $(p=0.11)(87)$. Whether high trough levels are causative or a consequence of nephrotoxicity remains to be determined. Since vancomycin is mainly excreted through glomerular filtration, a decrease in the glomerular filtration rate (GFR) would result in higher vancomycin serum concentrations. 
Among other risk factors that appear to be associated with an increased rate of nephrotoxicity are longer duration of vancomycin therapy and use of concomitant nephrotoxic medications (22). Nephrotoxic drugs include non-steroidal anti-inflammatory drugs (NSAIDs), inhibitors of the renin-angiotensin-aldosterone system (RAAS), loop diuretics, aminoglycosides, amphotericin $B$, colistin, immunosuppressant agents and intravenous contrast dye. Although nephrotoxicity resolves in $44-75 \%$ of the cases $(86,88)$, it is associated with a longer hospital length of stay and a higher mortality rate $(89,90)$.

For Col, data on risk factors for toxicity are limited compared to vancomycin Inl. Ingram et al. conducted a retrospective cohort study in 102 outpatients and found a nephrotoxicity rate of $15.7 \%$. Risk factors as determined in a multivariate analysis were hypertension, nephrotoxic drugs (more specifically aminoglycosides and loop diuretics) and a plateau concentration (Css) $\geq 28 \mathrm{mg} / \mathrm{L}$ (91). Spapen et al. also found that higher plateau concentrations (Css $>30 \mathrm{mg} / \mathrm{L}$ ) as well as longer duration of treatment increased the incidence of nephrotoxicity in ICU patients treated with Col. In addition, patients who developed nephrotoxicity were more likely to have additional risk factors associated with impaired renal function, such as diabetes and hypotensive shock (90). The risk of nephrotoxicity may be decreased with Col when compared to Inl. A recent meta-analysis compared nephrotoxicity of vancomycin Col versus $\mathrm{Inl}$ and showed a relative risk of 0.6 (Cl 0.4-0.9) (77). In addition, time to onset of nephrotoxicity appeared to be longer with Col compared to InI $(p=0.036)$ in a study by Ingram et al. (92). Hutschala et al. found no difference in nephrotoxicity between InI and Col. However, a rise of $1 \mathrm{mg} / \mathrm{L}$ in vancomycin concentration induced a rise in serum creatinine of $0.04 \mathrm{mg} / \mathrm{dL}$ with $\mathrm{Inl}$ versus a rise of $0.006 \mathrm{mg} / \mathrm{dL}$ with Col $(p<0.001)$ (93). In a retrospective cohort study, Saugel et al. showed that patients with Inl of vancomycin were more likely to need renal replacement therapy ( $23 \%$ versus $7 \%$ in Col; $p=0.007)$. Of note, the targeted trough level for Inl in this study was $5-10 \mathrm{mg} / \mathrm{L}$, which is lower than is currently advised. The targeted plateau concentration for Col was $15-25 \mathrm{mg} / \mathrm{L}(94)$. 


\section{In conclusion}

The IDSA guidelines recommend higher dosages in order to overcome the reduced susceptibility of MRSA to vancomycin (68). With higher dosing the risk of nephrotoxicity is likely to increase. Vancomycin Col has been suggested as an alternative approach to optimize the PK/PD of vancomycin. Current IDSA guidelines discourage the use of Col, since no benefit in terms of efficacy may be expected (68). The available literature suggests that in terms of efficacy vancomycin $\mathrm{Col}$ is neither superior nor inferior to vancomycin Inl. Treatment failure and mortality were comparable in both treatment regimens $(73,74,78,95)$. Furthermore, current literature suggests the risk of developing nephrotoxicity tends to be lower in vancomycin Col compared to Inl (77). Finally, continuous dosing of vancomycin, along with many other intravenously administered antibiotics, has shown to save health care costs and is easier to apply in home-care settings compared to intermittent dosing $(74,96)$. 


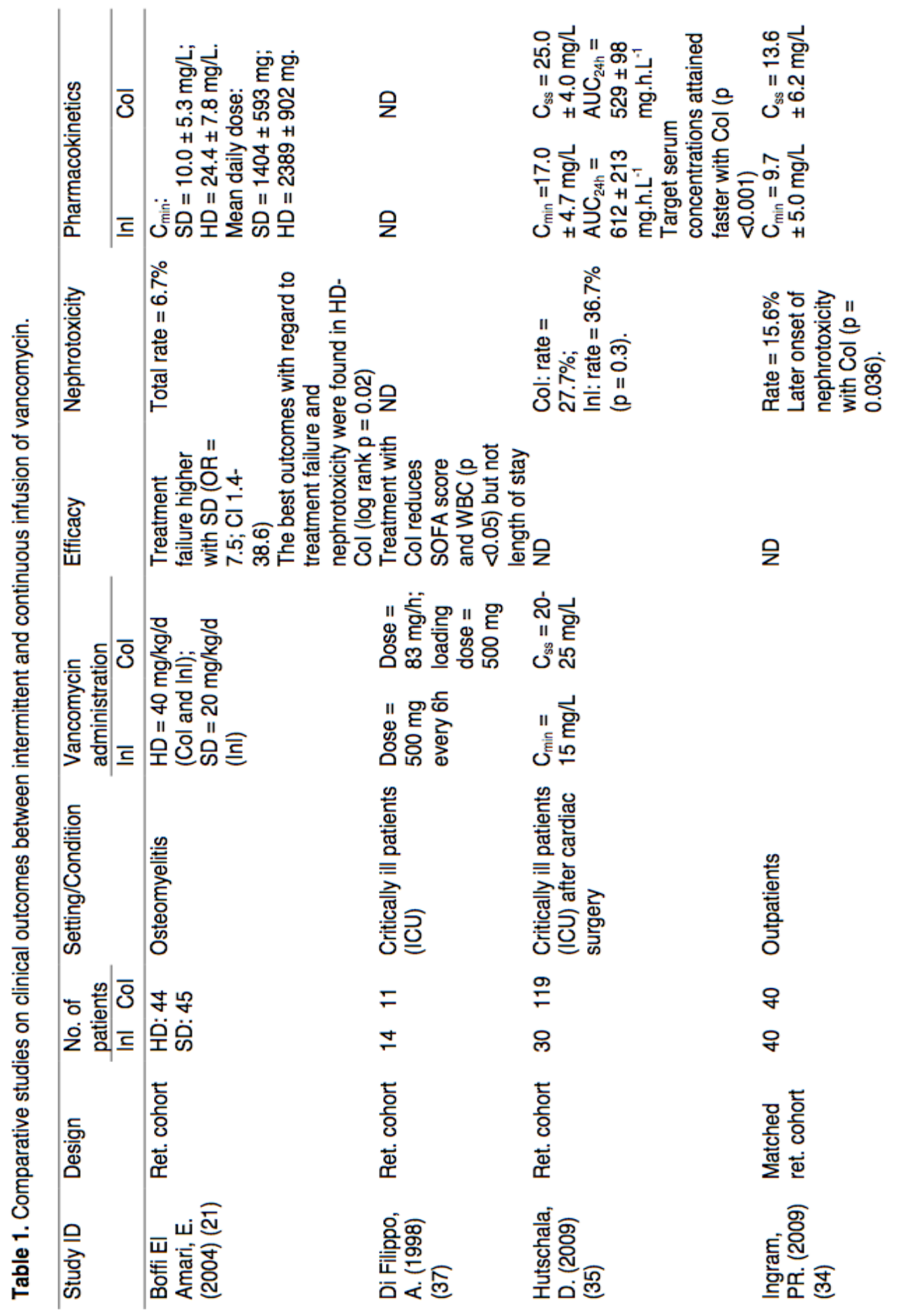




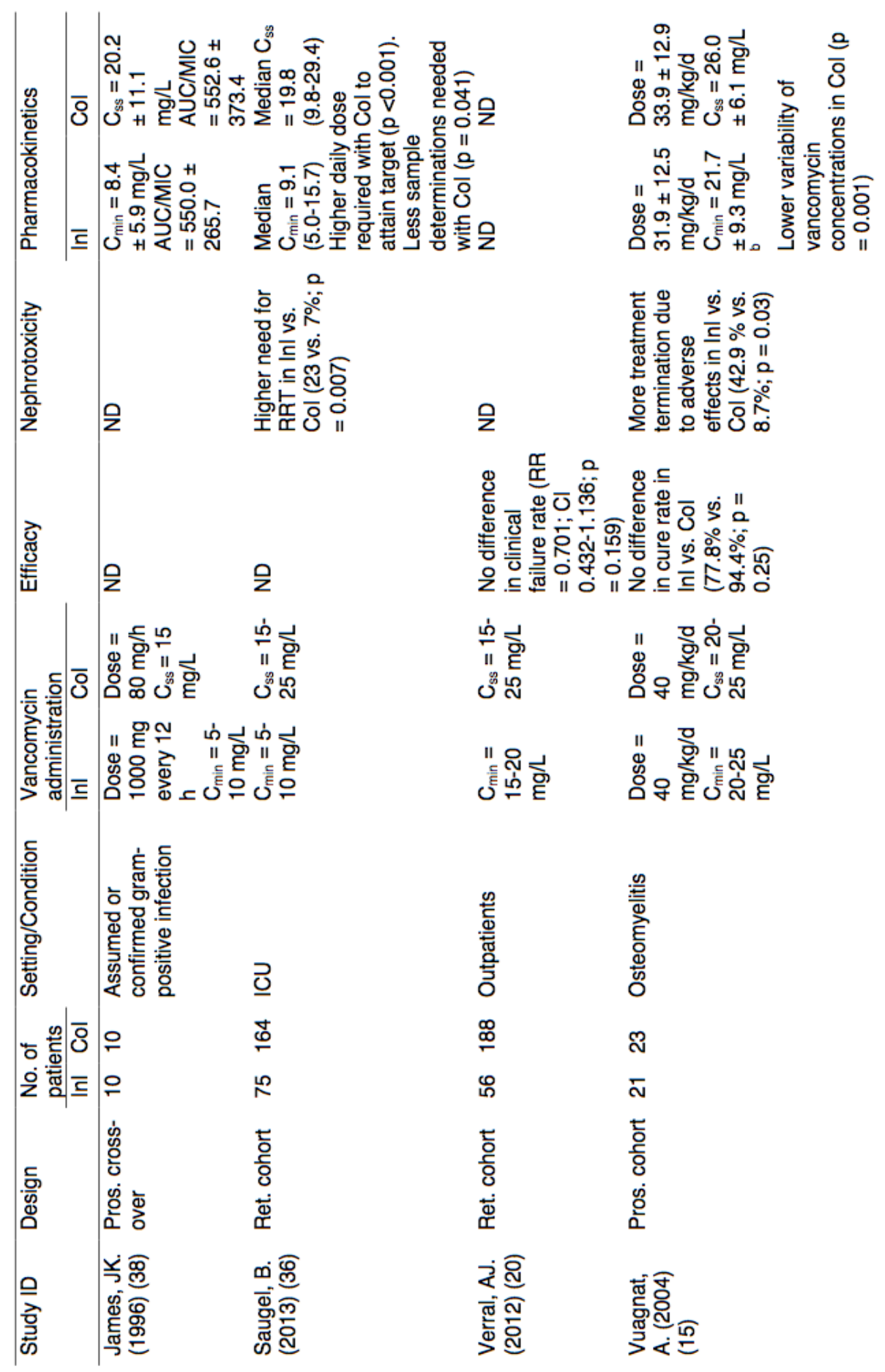




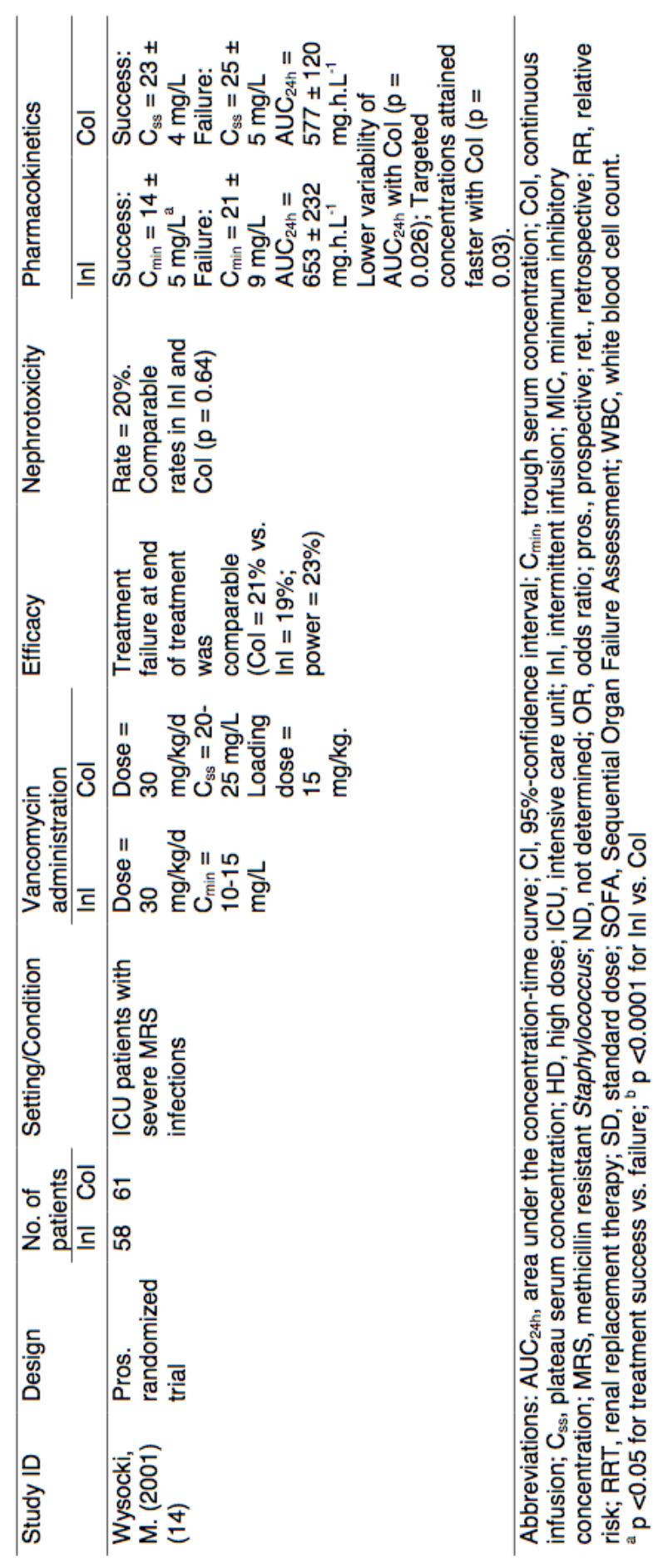


References

1. Avent ML, Rogers BA, Cheng AC, Paterson DL. Current use of aminoglycosides: I ndications, pharmacokinetics and monitoring for toxicity. Intern Med J 2011;41(6):441-9.

2. Pagkalis S, Mantadakis E, Mavros MN, Ammari C, Falagas ME. Pharmacological considerations for the proper clinical use of aminoglycosides. Drugs 2011;71(17):2277-94.

3. Drusano GL, Ambrose PG, Bhavnani SM, Bertino JS, Nafziger AN, Louie A. Back to the future: using aminoglycosides again and how to dose them optimally. Clin Infect Dis 2007;45(6):753-60.

4. Siegenthaler WE, Bonetti A, Luthy R. Aminoglycoside antibiotics in infectious diseases. An overview. Am J Med 1986;80(6B):2-14.

5. Hancock RE, Farmer SW, Li ZS, Poole K. Interaction of aminoglycosides with the outer membranes and purified lipopolysaccharide and OmpF porin of Escherichia coli. Antimicrob Agents Chemother 1991;35(7):1309-14.

6. Jana S, Deb JK. Molecular understanding of aminoglycoside action and resistance. Appl Microbiol Biotechnol 2006;70(2):140-50.

7. Bryan LE, Van den Elzen HM. Streptomycin accumulation in susceptible and resistant strains of Escherichia coli and Pseudomonas aeruginosa. Antimicrob Agents Chemother 1976;9(6):928-38.

8. Craig WA, Redington J, Ebert SC. Pharmacodynamics of amikacin in vitro and in mouse thigh and lung infections. J Antimicrob Chemother 1991;27 Suppl C:29-40.

9. Isaksson B, Nilsson L, Maller R, Soren L. Postantibiotic effect of aminoglycosides on gram-negative bacteria evaluated by a new method. J Antimicrob Chemother 1988;22(1):23-33.

10. Rescott DL, Nix DE, Holden P, Schentag JJ. Comparison of two methods for determining in vitro postantibiotic effects of three antibiotics on Escherichia coli. Antimicrob Agents Chemother 1988;32(4):450-3.

11. Begg EJ, Barclay ML. Aminoglycosides--50 years on. Br J Clin Pharmacol 1995;39(6):597-603.

12. Horrevorts AM, de Witte J, Degener JE, Dzoljic-Danilovic G, Hop WC, Driessen $\mathrm{O}$, et al. Tobramycin in patients with cystic fibrosis. Adjustment in dosing interval for effective treatment. Chest 1987;92(5):844-8.

13. Barclay ML, Begg EJ. Aminoglycoside toxicity and relation to dose regimen. Adverse Drug React Toxicol Rev 1994;13(4):207-34.

14. Martinez-Salgado C, Lopez-Hernandez FJ, Lopez-Novoa JM. Glomerular nephrotoxicity of aminoglycosides. Toxicol Appl Pharmacol 2007;223(1):86-98.

15. Mingeot-Leclercq MP, Tulkens PM. Aminoglycosides: nephrotoxicity. Antimicrob Agents Chemother 1999;43(5):1003-12.

16. Contrepois A, Brion N, Garaud JJ, Faurisson F, Delatour F, Levy JC, et al. Renal disposition of gentamicin, dibekacin, tobramycin, netilmicin, and amikacin in humans. Antimicrob Agents Chemother 1985;27(4):520-4.

17. Lopez-Novoa JM, Quiros Y, Vicente L, Morales Al, Lopez-Hernandez FJ. New insights into the mechanism of aminoglycoside nephrotoxicity: an integrative point of view. Kidney Int 2011;79(1):33-45.

18. Quiros Y, Vicente-Vicente L, Morales AI, Lopez-Novoa JM, Lopez-Hernandez FJ. An integrative overview on the mechanisms underlying the renal tubular cytotoxicity of gentamicin. Toxicol Sci 2011;119(2):245-56. 
19. Huth ME, Ricci AJ, Cheng AG. Mechanisms of aminoglycoside ototoxicity and targets of hair cell protection. Int J Otolaryngol 2011;2011:937861.

20. Matz GJ. Aminoglycoside cochlear ototoxicity. Otolaryngol Clin North Am 1993;26(5):705-12.

21. Fee WE, Jr. Aminoglycoside ototoxicity in the human. Laryngoscope 1980;90(10 Pt 2 Suppl 24):1-19.

22. Mulheran M, Degg C. Comparison of distortion product OAE generation between a patient group requiring frequent gentamicin therapy and control subjects. $\mathrm{Br} \mathrm{J}$ Audiol 1997;31(1):5-9.

23. Xie J, Talaska AE, Schacht J. New developments in aminoglycoside therapy and ototoxicity. Hear Res 2011;281(1-2):28-37.

24. Zheng Y, Schachern PA, Sone M, Papapella MM. Aminoglycoside ototoxicity. Otol Neurotol 2001;22(2):266-8.

25. Rubio TT. Infection in patients with cystic fibrosis. Am J Med 1986;81(1A):737.

26. Prayle A, Smyth AR. Aminoglycoside use in cystic fibrosis: therapeutic strategies and toxicity. Curr Opin Pulm Med 2010;16(6):604-10.

27. Doring G, Conway SP, Heijerman HG, Hodson ME, Hoiby N, Smyth A, et al. Antibiotic therapy against Pseudomonas aeruginosa in cystic fibrosis: a European consensus. Eur Respir J 2000;16(4):749-67.

28. Touw DJ. Clinical pharmacokinetics of antimicrobial drugs in cystic fibrosis. Pharm World Sci 1998;20(4):149-60.

29. Levy J, Smith AL, Koup JR, Williams-Warren J, Ramsey B. Disposition of tobramycin in patients with cystic fibrosis: a prospective controlled study. J Pediatr 1984;105(1):117-24.

30. Smyth AR, Bhatt J. Once-daily versus multiple-daily dosing with intravenous aminoglycosides for cystic fibrosis. Cochrane Database Syst Rev 2012;2:CD002009.

31. Smyth A, Tan KH, Hyman-Taylor P, Mulheran M, Lewis S, Stableforth D, et al. Once versus three-times daily regimens of tobramycin treatment for pulmonary exacerbations of cystic fibrosis--the TOPIC study: a randomised controlled trial. Lancet 2005;365(9459):573-8.

32. Prescott WA, Jr., Nagel JL. Extended-interval once-daily dosing of aminoglycosides in adult and pediatric patients with cystic fibrosis. Pharmacotherapy 2010;30(1):95-108.

33. Young DC, Zobell JT, Stockmann C, Waters CD, Ampofo K, Sherwin CM, et al. Optimization of anti-pseudomonal antibiotics for cystic fibrosis pulmonary exacerbations: V. Aminoglycosides. Pediatr Pulmonol 2013;48(11):1047-61.

34. Prayle A, Watson A, Fortnum H, Smyth A. Side effects of aminoglycosides on the kidney, ear and balance in cystic fibrosis. Thorax 2010;65(7):654-8.

35. Radigan EA, Gilchrist NA, Miller MA. Management of aminoglycosides in the intensive care unit. J Intensive Care Med 2010;25(6):327-42.

36. Boucher BA, Wood GC, Swanson JM. Pharmacokinetic changes in critical illness. Crit Care Clin 2006;22(2):255-71, vi.

37. Power BM, Forbes AM, van Heerden PV, llett KF. Pharmacokinetics of drugs used in critically ill adults. Clin Pharmacokinet 1998;34(1):25-56.

38. Buijk SE, Mouton JW, Gyssens IC, Verbrugh HA, Bruining HA. Experience with a once-daily dosing program of aminoglycosides in critically ill patients. Intensive Care Med 2002;28(7):936-42. 
39. Wallace AW, Jones M, Bertino JS, Jr. Evaluation of four once-daily aminoglycoside dosing nomograms. Pharmacotherapy 2002;22(9):1077-83.

40. Rea RS, Capitano B, Bies R, Bigos KL, Smith R, Lee H. Suboptimal aminoglycoside dosing in critically ill patients. Ther Drug Monit 2008;30(6):674-:1.

41. Stoll BJ, Gordon T, Korones SB, Shankaran S, Tyson JE, Bauer CR, et al. Early-onset sepsis in very low birth weight neonates: a report from the National Institute of Child Health and Human Development Neonatal Research Network. J Pediatr 1996;129(1):72-80.

42. Koren G. Therapeutic drug monitoring principles in the neonate. National Academy of CLinical Biochemistry. Clin Chem 1997;43(1):222-7.

43. Allegaert K, Anderson BJ. Interindividual variability of aminoglycoside pharmacokinetics in preterm neonates at birth. Eur J Clin Pharmacol 2006;62(12):1011-2.

44. Besunder JB, Reed MD, Blumer JL. Principles of drug biodisposition in the neonate. A critical evaluation of the pharmacokinetic-pharmacodynamic interface (Part I). Clin Pharmacokinet 1988;14(4):189-216.

45. Touw DJ, Westerman EM, Sprij AJ. Therapeutic drug monitoring of aminoglycosides in neonates. Clin Pharmacokinet 2009;48(2):71-88.

46. Begg EJ, Vella-Brincat JW, Robertshawe B, McMurtrie MJ, Kirkpatrick CM, Darlow B. Eight years' experience of an extended-interval dosing protocol for gentamicin in neonates. J Antimicrob Chemother 2009;63(5):1043-9.

47. Sum BL VS, Westerman EM, Sprij AJ, Touw DJ. An extended-interval gentamicin dosage regimen in newborns: a propsective study. European Journal of Hospital Pharmacy Science 2007;13(4):98-104.

48. Veltkamp SA, Westerman EM, Sprij AJ, Sum BL TD. Gentamicin in preterm neonates: an extended interval dosing schedule. European Journal of Hospital Pharmacy Science 2007;13(4):92-97.

49. de Hoog M, Mouton JW, Schoemaker RC, Verduin CM, van den Anker JN. Extended-interval dosing of tobramycin in neonates: implications for therapeutic drug monitoring. Clin Pharmacol Ther 2002;71(5):349-58.

50. de Hoog M, Schoemaker RC, Mouton JW, van den Anker JN. Tobramycin population pharmacokinetics in neonates. Clin Pharmacol Ther 1997;62(4):392-9.

51. Rao SC, Srinivasjois R, Hagan R, Ahmed M. One dose per day compared to multiple doses per day of gentamicin for treatment of suspected or proven sepsis in neonates. Cochrane Database Syst Rev 2011(11):CD005091.

52. Best EJ, Gazarian M, Cohn R, Wilkinson M, Palasanthiran P. Once-daily gentamicin in infants and children: a prospective cohort study evaluating safety and the role of therapeutic drug monitoring in minimizing toxicity. Pediatr Infect Dis J 2011;30(10):827-32.

53. Fanos V, Dall'Agnola A. Antibiotics in neonatal infections: a review. Drugs 1999;58(3):405-27.

54. Rybak MJ. The pharmacokinetic and pharmacodynamic properties of vancomycin. Clinical infectious diseases: an official publication of the Infectious Diseases Society of America 2006;42 Suppl 1:S35.

55. EuroCept BV. Vancocin. Summary of Product Characteristics. In.

56. Levine DP. Vancomycin: a history. Clinical infectious diseases: an official publication of the Infectious Diseases Society of America 2006;42 Suppl 1:S5.

57. Moise-Broder PA, Forrest A, Birmingham MC, Schentag JJ. Pharmacodynamics of vancomycin and other antimicrobials in patients with Staphylococcus aureu 
lower respiratory tract infections. Clin Pharmacokinet 2004;43(13):925-42.

58. Rybak MJ. The pharmacokinetic and pharmacodynamic properties of vancomycin. Clin Infect Dis 2006;42 Suppl 1:S35-9.

59. Tenover FC, Biddle JW, Lancaster MV. Increasing resistance to vancomycin and other glycopeptides in Staphylococcus aureus. Emerg Infect Dis 2001;7(2):327-32.

60. Liu C, Bayer A, Cosgrove SE, Daum RS, Fridkin SK, Gorwitz RJ, et al. Clinical practice guidelines by the infectious diseases society of america for the treatment of methicillin-resistant Staphylococcus aureus infections in adults and children: executive summary. Clin Infect Dis 2011;52(3):285-92.

61. Rybak M, Lomaestro B, Rotschafer JC, Moellering R, Jr., Craig W, Billeter M, et al. Therapeutic monitoring of vancomycin in adult patients: a consensus review of the American Society of Health-System Pharmacists, the Infectious Diseases Society of America, and the Society of Infectious Diseases Pharmacists. Am J Health Syst Pharm 2009;66(1):82-98.

62. Tenover FC, Moellering RC, Jr. The rationale for revising the Clinical and Laboratory Standards Institute vancomycin minimal inhibitory concentration interpretive criteria for Staphylococcus aureus. Clinical infectious diseases: an official publication of the Infectious Diseases Society of America 2007;44(9):1208.

63. Jacob JT, DiazGranados CA. High vancomycin minimum inhibitory concentration and clinical outcomes in adults with methicillin-resistant Staphylococcus aureus infections: a meta-analysis. International journal of infectious diseases: IJID: official publication of the International Society for Infectious Diseases;17(2):e93.

64. Mavros MN, Tansarli GS, Vardakas KZ, Rafailidis PI, Karageorgopoulos $\mathrm{DE}$, Falagas ME. Impact of vancomycin minimum inhibitory concentration on clinical outcomes of patients with vancomycin-susceptible Staphylococcus aureus infections: a meta-analysis and meta-regression. International journal of antimicrobial agents;40(6):496.

65. van Hal SJ, Lodise TP, Paterson DL. The clinical significance of vancomycin minimum inhibitory concentration in Staphylococcus aureus infections: a systematic review and meta-analysis. Clinical infectious diseases: an official publication of the Infectious Diseases Society of America;54(6):755.

66. Hidayat LK, Hsu DI, Quist R, Shriner KA, Wong-Beringer A. High-dose vancomycin therapy for methicillin-resistant Staphylococcus aureus infections: efficacy and toxicity. Arch Intern Med 2006;166(19):2138-44.

67. Pea F, Viale P. Pharmacodynamics of antibiotics to treat multidrug-resistant Gram-positive hospital infections. Expert Rev Anti Infect Ther 2007;5(2):255-70.

68. Rybak MJ, Lomaestro BM, Rotschafer JC, Moellering RC, Craig WA, Billeter $M$, et al. Vancomycin therapeutic guidelines: a summary of consensus recommendations from the infectious diseases Society of America, the American Society of Health-System Pharmacists, and the Society of Infectious Diseases Pharmacists. Clin Infect Dis 2009;49(3):325-7.

69. Vandecasteele SJ, De Vriese AS, Tacconelli E. The pharmacokinetics and pharmacodynamics of vancomycin in clinical practice: evidence and uncertainties. J Antimicrob Chemother;68(4):743-8.

70. Chua K, Howden BP. Treating Gram-positive infections: vancomycin update and the whys, wherefores and evidence base for continuous infusion of anti-Gram-positive antibiotics. Current opinion in infectious diseases 2009;22(6):525.

71. Rybak MJ, Lomaestro BM, Rotschafer JC, Moellering RC, Jr., Craig WA, 
Billeter M, et al. Therapeutic monitoring of vancomycin in adults summary of consensus recommendations from the American Society of Health-System Pharmacists, the Infectious Diseases Society of America, and the Society of Infectious Diseases Pharmacists. Pharmacotherapy 2009;29(11):1275-9.

72. del Mar Fernandez de Gatta Garcia M, Revilla N, Calvo MV, Dominguez-Gil A, Sanchez Navarro A. Pharmacokinetic/pharmacodynamic analysis of vancomycin in ICU patients. Intensive care medicine 2007;33(2):279.

73. Vuagnat A, Stern R, Lotthe A, Schuhmacher H, Duong M, Hoffmeyer P, et al. High dose vancomycin for osteomyelitis: continuous vs. intermittent infusion. J Clin Pharm Ther 2004;29(4):351-7.

74. Wysocki M, Delatour F, Faurisson F, Rauss A, Pean Y, Misset B, et al. Continuous versus intermittent infusion of vancomycin in severe Staphylococcal infections: prospective multicenter randomized study. Antimicrob Agents Chemother 2001;45(9):2460-7.

75. Ampe E, Delaere B, Hecq JD, Tulkens PM, Glupczynski Y. Implementation of a protocol for administration of vancomycin by continuous infusion: pharmacokinetic, pharmacodynamic and toxicological aspects. International journal of antimicrobial agents;41(5):439.

76. Pea F, Furlanut M, Negri C, Pavan F, Crapis M, Cristini F, et al. Prospectively validated dosing nomograms for maximizing the pharmacodynamics of vancomycin administered by continuous infusion in critically ill patients. Antimicrob Agents Chemother 2009;53(5):1863-7.

77. $\quad$ Cataldo M.A., Tacconelli E., Grilli E., Pea F., Petrosillo N. Continuous versus intermittent infusion of vancomycin for the treatment of Gram-positive infections: systematic review and meta-analysis. J Antimicrob Chemother. 2012;67(1):17-24.

78. Verrall AJ, Llorin R, Tam VH, Lye DC, Sulaiman Z, Zhong L, et al. Efficacy of continuous infusion of vancomycin for the outpatient treatment of methicillin-resistant Staphylococcus aureus infections. J Antimicrob Chemother 2012;67(12):2970-3.

79. Boffi El Amari E., Vuagnat A., Stern R., Assal M., Denormandie P., Hoffmeyer P., Bernard L. High versus standard dose vancomycin for osteomyelitis. Scand J Infect Dis 2004;36(10):712-7.

80. van Hal SJ, Paterson DL, Lodise TP. Systematic review and meta-analysis of vancomycin-induced nephrotoxicity associated with dosing schedules that maintain troughs between 15 and 20 milligrams per liter. Antimicrobial Agents and Chemotherapy:57(2):734.

81. Bentley ML. Acute kidney insufficiency in the critically ill. Journal of pharmacy practice 2011;24(1):61-69.

82. Bosso JA, Nappi J, Rudisill C, Wellein M, Bookstaver PB, Swindler J, et al. Relationship between vancomycin trough concentrations and nephrotoxicity: a prospective multicenter trial. Antimicrob Agents Chemother 2011;55(12):5475-9.

83. Cano EL, Haque NZ, Welch VL, Cely CM, Peyrani P, Scerpella EG, et al. Incidence of nephrotoxicity and association with vancomycin use in intensive care unit patients with pneumonia: retrospective analysis of the IMPACT-HAP Database. Clinical therapeutics 2011;34(1):149-157.

84. Jeffres MN, Isakow W, Doherty JA, Micek ST, Kollef MH. A retrospective analysis of possible renal toxicity associated with vancomycin in patients with health care-associated methicillin-resistant Staphylococcus aureus pneumonia. Clinical therapeutics 2007;29(6):1107. 
85. Lodise TP, Graves J, Evans A, Graffunder E, Helmecke M, Lomaestro BM, et al. Relationship between vancomycin MIC and failure among patients with methicillin-resistant Staphylococcus aureus bacteremia treated with vancomycin. Antimicrob Agents Chemother 2008;52(9):3315-20.

86. van Hal SJ, Paterson DL, Lodise TP. Systematic review and meta-analysis of vancomycin-induced nephrotoxicity associated with dosing schedules that maintain troughs between 15 and 20 milligrams per liter. Antimicrobial Agents and Chemotherapy 2013;57(2):734-744.

87. Lodise TP, Patel N, Lomaestro BM, Rodvold KA, Drusano GL. Relationship between initial vancomycin concentration-time profile and nephrotoxicity among hospitalized patients. Clin Infect Dis 2009;49(4):507-14.

88. Ramirez E, Jimenez C, Borobia AM, Tong HY, Medrano N, Krauel-Bidwell L, et al. Vancomycin-induced acute kidney injury detected by a prospective pharmacovigilance program from laboratory signals. Therapeutic drug monitoring 2013;35(3):360366.

89. Cianferoni S, Devigili A, Ocampos-Martinez E, Penaccini L, Scolletta S, Abdelhadii $A$, et al. Development of acute kidney injury during continuous infusion of vancomycin in septic patients. Infection 2013;41(4):811-820.

90. Spapen HD, Janssen van Doorn K, Diltoer M, Verbrugghe W, Jacobs R, Dobbeleir $\mathrm{N}$, et al. Retrospective evaluation of possible renal toxicity associated with continuous infusion of vancomycin in critically ill patients. Ann Intensive Care 2011;1(1):26. 91. Ingram PR, Lye DC, Fisher DA, Goh WP, Tam VH. Nephrotoxicity of continuous versus intermittent infusion of vancomycin in outpatient parenteral antimicrobial therapy. Int J Antimicrob Agents 2009;34(6):570-4.

92. Ingram PR, Lye DC, Tambyah PA, Goh WP, Tam VH, Fisher DA. Risk factors for nephrotoxicity associated with continuous vancomycin infusion in outpatient parenteral antibiotic therapy. The Journal of antimicrobial chemotherapy 2008;62(1):168.

93. Hutschala D, Kinstner C, Skhirdladze K, Thalhammer F, Muller M, Tschernko E. Influence of vancomycin on renal function in critically ill patients after cardiac surgery: continuous versus intermittent infusion. Anesthesiology 2009;111(2):356-65.

94. Saugel B, Nowack MC, Hapfelmeier A, Umgelter A, Schultheiss C, Thies P, et al. Continuous intravenous administration of vancomycin in medical intensive care unit patients. Journal of critical care 2013;28(1):9-13.

95. Boffi El Amari E, Vuagnat A, Stern R, Assal M, Denormandie P, Hoffmeyer $P$, et al. High versus standard dose vancomycin for osteomyelitis. Scand J Infect Dis 2004;36(10):712-7.

96. van Zanten AR, Engelfriet PM, van Dillen K, van Veen M, Nuijten MJ, Polderman $\mathrm{KH}$. Importance of nondrug costs of intravenous antibiotic therapy. Crit Care 2003;7(6):R184-90. 


\section{Aim of this thesis}

The aim of this thesis is to explore and establish new dosing strategies for intravenous aminoglycosides and vancomycin therapies with an optimal balance between efficacy and toxicity.

Outline of this thesis

\section{Chapter 2. Aminoglycosides}

Chapter 2.1 describes a randomized clinical trial investigating the effects of the time of administration on tobramycin pharmacokinetics and nephrotoxicity in adult cystic fibrosis patients.

Chapter 2.2 reports retrospective data on the chronopharmacology of aminoglycosides in general ward and critically ill patients.

Chapter 2.3 compares the nephrotoxicity of tobramycin and gentamicin in a population of clinically infected patients.

Chapter 2.4 provides information on a prospectively tested new gentamicin dosing algorithm in neonates admitted to a special care nursery. 


\section{Chapter 3. Vancomycin}

Chapter 3.1 reviews and compares vancomycin exposure variabilities of continuous and intermittent dosing regimens in adult hospitalized patients.

In Chapter 3.2 the exposure variability of vancomycin in a population of critically ill patients on continuous or intermittent infusion is studied.

Chapter 3.3 shows a method to design and validate a dosing regimen of continuously dosed vancomycin in a mixed general ward and intensive care unit population using historical within-population data.

Chapter 4 summarizes and discusses the results and conclusions of the conducted studies. Moreover, their impact on clinical practice is addressed and suggestions for future studies are provided with respect to aminoglycosides and vancomycin therapies in clinically infected patient populations. 


\section{Chapter 2}

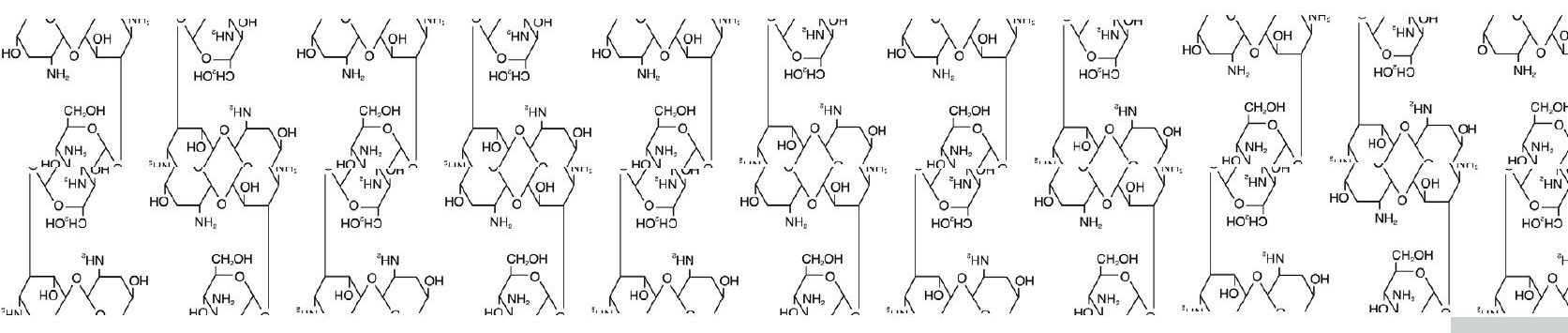





\title{
Chapter 2.1
}

\section{The Effect of the Circadian Rhythm on the Pharmacokinetics} of Once Daily Tobramycin in Cystic Fibrosis Patients

\author{
E.M. VAN MAARSEVEEN, \\ R. VAN DER MEER \\ C. NEEF \\ H.G.M. HEIJERMAN \\ D.J. TOUW
}

submitted
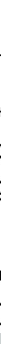
In the era of multiple daily dosing of systemic aminoglycosides a circadian rhythm in the clearance of these vital antibiotics has been demonstrated in animals and healthy volunteers. Over the past two decades, however, once daily dosing (ODD) regimens proved to be less nephrotoxic and therefore ODD protocols were adopted by centers worldwide for most indications requiring treatment with an aminoglycoside. In this study the effect of the time of administration on the pharmacokinetics of once-daily tobramycin in adult CF patients was investigated. In this open randomized study CF patients were administered intravenous tobramycin at 8:00 or at 22:00. Pharmacokinetic parameter values and biochemical parameters of renal function were compared between groups. Twenty-five patients were included. Mean weight-corrected clearances of tobramycin were 1.46 vs. $1.43 \mathrm{~mL} / \mathrm{min}^{*} \mathrm{~kg}(p=0.50)$ and mean volumes of distribution were $0.25 \mathrm{vs} .0 .27 \mathrm{~L} / \mathrm{kg}(p=0.54)$ for the 8:00 and 22:00 groups, respectively. Furthermore, no significant differences were detected in the changes in estimated creatinine clearance or tobramycin clearance from day 1 to day 8 in the 8:00 group or in the 22:00 group indicating there was no decline in clearance over time. At day 8 of therapy the rise in serum BUN in the 22:00 group was significant higher compared to the $8: 00$ group (1.8 vs. $0.2 \mathrm{mmol} / \mathrm{L}, \mathrm{p}=0.015$ ). In conclusion, the time of administration did not affect tobramycin pharmacokinetics the adult CF population studied. The rise in serum BUN in the 22:00 group deserves further exploration.

\section{Introduction}

Cystic fibrosis is an incurable, genetically inherited disease that causes changes in the ion transport mechanisms in different parts of the body (lungs, skin). Although the disease affects several body systems it is respiratory decline that leads to fatality (1). Patients with CF are prone to acquire pulmonary infections with Pseudomonas aeruginosa and other micro organisms which are associated with irreversible pulmonary damage leading to a reduced life expectancy (2). Intravenous aminoglycosides, in combination with beta-lactam antibiotics, are a cornerstone for the treatment of exacerbations of chronic pulmonary infections (3) caused by Pseudomonas aeruginosa.

However, frequent use of intravenous aminoglycosides is associated with nephro- and ototoxicity and in some cases with vestibulotoxicity 
$(4,5)$. Repeated or prolonged dosing may cause damage to the proximal tubuli, resulting in renal impairment, which affects up to $42 \%$ of adult CF patients (6). As the life expectancy of CF patients is extending as a consequence of improved treatment, cumulative toxicity caused by aminoglycosides is nowadays more relevant than in the past. The TOPIC study demonstrated that once-daily dosing (ODD) of tobramycin is at least as effective and may be less toxic compared to multiple daily dosing (MDD) in CF patients (7). Hence, ODD of tobramycin has been adopted by a great number of cystic fibrosis treatment centers worldwide. Since aminoglycosides are almost completely excreted into the urine by glomerular filtration, their kinetics may fluctuate with diurnal changes in renal function as previously demonstrated in animal experiments $(8,9)$, and in healthy volunteers $(8,10)$. Consequently, if an effect of the administration on the pharmacokinetics and toxicity of tobramycin exists in CF patients, then clinically it will be more relevant in ODD compared to MDD regimens. Human data on a circadian rhythm in pharmacokinetics are however conflicting and whether or not an association is found primarily seem depend on dosing regimen and the specific population investigated (11-13). A post-hoc analysis of the TOPIC study performed denoted a peculiar difference of $30 \%$ in renal elimination between the ODD group and the three times daily group (14). The authors relate this to the fact that the majority of patients in the ODD group (53 of 71) received their active dose in the evening. Therefore, most of the systemic drug exposure occurred during the resting period when renal function is relatively decreased. In this study a decreased renal elimination for the pediatric as well as the adult once daily group was observed. One could hypothesize this to be the result of a circadian rhythm in drug clearance caused by either active and resting periods or dietary protein intake or both. To date, the effect of diurnal changes on aminoglycoside pharmacokinetics in CF patients has not been studied prospectively. Ultimately, it is important that CF patients are exposed to aminoglycosides at the time period of highest clearance to prevent acute adverse events and events resulting from accumulated toxicity without compromising efficacy $(15,16)$. The present randomized clinical study was designed to evaluate diurnal changes in tobramycin pharmacokinetics and renal function. 


\section{Patients \& Methods}

In this open randomised clinical trial ((Dutch Trial Register number: NTR3309) patients with cystic fibrosis, who were treated with intravenous tobramycin for a pulmonary exacerbation at the Haga Teaching Hospital (The Hague, The Netherlands), were eligible for inclusion. The study was approved by the local Medical Ethics Committee. The study was powered to detect a $25 \%$ difference in tobramycin exposure quantified by the area-under-the-concentration-time-curve (AUC), since regulatory authorities consider $80-125 \%$ limits to be a clinically relevant cut-offs for bioequivalence. An informed consent was obtained from all participants. The inclusion criteria were: age older than 18 , a diagnosis of cystic fibrosis (i.e., sweat chloride $>60 \mathrm{mmol} / \mathrm{l}$ or a genotype known to cause the disease), chronic infection with Pseudomonas aeruginosa with the most recently isolated organism showing sensitivity to tobramycin and pulmonary exacerbation as defined by Fuchs and colleagues (17). Patients who were on other nephrotoxic drugs or were allergic to aminoglycosides, had granulocytopenia ( $<1.0 \times 109 / L)$, were pregnant, had estimated GFR $<40 \mathrm{ml} / \mathrm{min}$ or pre-existing hearing impairment, were excluded. After inclusion participants were randomly assigned to receive tobramycin at either 8:00 or at 22:00. Total daily doses equivalent to those previously received by patients during routine treatment were used. If a patient had not previously been treated with tobramycin, a dose of $10 \mathrm{mg} / \mathrm{kgTBW} /$ day was administered as a $30 \mathrm{~min}$ infusion (TBW is total body weight). Tobramycin concentrations were drawn one hour after start of the infusion (peak level) and between 6-8 $\mathrm{h}$ after start of the infusion (mid level). Dosage regimes were altered, if serum concentrations were outside target values (peak: $25-30 \mathrm{mg} / \mathrm{L}$; extrapolated trough $<0.5 \mathrm{mg} / \mathrm{L}$ ). Samples were analyzed using a fluorescence polarization immunoassay (AxSym, Abbott, Illinois, U.S.A.). An assay error of standard deviation $=0.25+0.15^{\star} \mathrm{C}$ (where $\mathrm{C}$ is the concentration measured) was used in the pharmacokinetic analysis. Tobramycin pharmacokinetic parameters at days 1 and 8 were calculated for each patient using a previously described one-compartment model with linear pharmacokinetics (18) using the MW/Pharm pharmacokinetic software package version 3.60 (19). 
Pharmacokinetic parameters that were determined included apparent volume of distribution $(\mathrm{Vd})$, tobramycin clearance (Cl_Tob) and area-under-the-concentration-time-curve (AUC).

Serum creatinine concentrations, $24 \mathrm{~h}$-urine collections and blood urine nitrogen (BUN) were taken in the morning on days 1 and 8 of tobramycin treatment. Serum and urinary creatinine were analyzed using a colorimetric method and BUN by a kinetic enzymatic assay (DxC, Beckman Coulter Inc., Brea, CA, USA). Subsequently, renal function was quantified at baseline and at day 8 using serum creatinine concentrations to estimate the creatinine clearance using the formula proposed by Jelliffe (20), which approximates the glomerular filtration rate (eGFR). Glomular filtration was also measured GFR (mGFR) using 24h-urine collections. The effect of the time period of administration of tobramycin on renal function was assessed by the change in serum BUN, eGFR and mGFR at day 8 compared to baseline.

\section{Statistical analysis}

A Kolmogorov-Smirnov test was used to identify normal distribution of data. Means and standard deviations of continuous variables are presented for normal distributions. Otherwise, medians and ranges were reported. A Student's T-test was used to determine statistical differences among normally distributed data. If data were not normally distributed, a Mann-Whitney-U test was performed. A Chi-square test was used to compare dichotomous variables between groups. $\mathrm{Vd}$, and $\mathrm{Cl}$ _Tob were analyzed after correction for TBW and AUCs were normalized to an administered dose of $500 \mathrm{mg}$ tobramycin (500mgTOB). Tests were two-tailed and a p-value of $<0.05$ was considered statistically significant. All Statistical analysis were performed using GraphPad Prism version 5.03 for Window (GraphPadSoftware, San Diego, CA, USA).

\section{Results}

\section{Study participants}

Twenty-five patients, who received at least one dose of tobramycin between July 2008 and May 2011, were enrolled in the study. No significant differences in baseline characteristics between the 8:00 and 22:00 groups were detected (Table 1). 
Table 1. Patient characteristics

\begin{tabular}{|cccc|}
\hline & $8: 00$ & $22: 00$ & p-value \\
\hline No. patients & 12 & 13 & \\
age $(\mathrm{yr})$ & $30(12)$ & $30(11)$ & $0.42^{\mathrm{a}}$ \\
male gender $(\%)$ & 63.6 & 72.7 & $0.87^{\mathrm{c}}$ \\
weight $(\mathrm{kg})$ & $60(21)$ & $61(22)$ & $1.0^{\mathrm{a}}$ \\
serum creatinine $(\mathrm{nmol} / \mathrm{mL})$ & $66(55-97)$ & $65(33-91)$ & $0.46^{\mathrm{b}}$ \\
eGFR $(\mathrm{mL} / \mathrm{min})$ & $118(67-132)$ & $112(82-175)$ & $0.60^{\mathrm{b}}$ \\
cGFR $(\mathrm{ml} / \mathrm{min})$ & $102(75-145)$ & $108(55-185)$ & $1.0^{\mathrm{b}}$ \\
BUN $(\mathrm{nmol} / \mathrm{mL})$ & $4.2(2.2-7.7)$ & $4.0(2.3-6.3)$ & $0.27^{\mathrm{b}}$ \\
& & & \\
\hline
\end{tabular}

Baseline characteristics of 8:00 and 22:00 groups are shown. Dichotomous variables were expressed as percentages and continuous variables as means or medians with standard deviations or ranges abetween parentheses. Abbreviations are eGFR, estimated glomerular filtration rate; BUN, blood urea nitrogen. a Student's T-test, b Mann-Whitney test, c Chi-square test

Treatment was either stopped or continued at home after discharge for 2 patients during the first week of tobramycin therapy in both study arms. Tobramycin dose was adjusted at day 1 in 3 patients in the 8:00 group and in 5 patients in the 22:00 group. The median cumulative doses over 7 days of tobramycin therapy were 3980 and $3840 \mathrm{mg}$ $(p=0.90)$ for the 8:00 and 22:00 groups respectively. Patients did not receive any other nephrotoxic medication from day 1 to day 8 .

\section{Pharmacokinetics}

No significant differences in pharmacokinetic parameters were detected between the 8:00 and 22:00 groups (Table 2 and Figure 1). Furthermore, no significant difference was detected in the change in Cl_Tob between day 1 and day 8 in the $8: 00$ group $(p=0.58)$ or in the 22:00 group $(p=0.67)$ indicating there was no decline in clearance over time. 


\begin{abstract}
Parameters of renal function
No significant difference in the change in eGFR from baseline was found between the $8: 00$ and 22:00 groups ( 3.8 vs. $10.0 \mathrm{~mL} / \mathrm{h}$ respectively, $p=1.0$ ) (Figure 2). The rise in serum BUN in the 22:00 group was statistically significant compared to the $8: 00$ group $(1.8 \mathrm{vs} .0 .2 \mathrm{mmol} / \mathrm{L}$ respectively, $p=0.015$ ) and serum BUN at day 8 was significantly higher compared to baseline in the 22:00 group ( $p=0.014)$, but not in the 8:00 group ( $p=0.79$ ). The change in mGFR could not be analyzed due to incomplete $24 \mathrm{~h}$-urine sampling in 7 of 25 participants at day 8 .
\end{abstract}


Table 2. Dosing and pharmacokinetic parameters

\begin{tabular}{ccccc}
\hline & \multicolumn{2}{c}{ time of administration } & & \\
\cline { 1 - 2 } Day 1 & $8: 00$ & $22: 00$ & Rate ratio & p-value \\
\hline Dose $(\mathrm{mg})$ & $525(420-600)$ & $500(400-600)$ & n.a. & $0.35^{\mathrm{b}}$ \\
Cmax (mg/L) & $35.0(3.9)$ & $30.4(4.1)$ & 1.15 & $0.53^{\mathrm{a}}$ \\
AUC (mg*h/L) & $87(26)$ & $97(29)$ & 0.90 & $0.21^{\mathrm{a}}$ \\
Vd $(\mathrm{L} / \mathrm{kg})$ & $0.25(0.08)$ & $0.27(0.09)$ & 0.93 & $0.54^{\mathrm{a}}$ \\
Cl_TOB $(\mathrm{mL} / \mathrm{min} / \mathrm{kg})$ & $1.46(0.44)$ & $1.43(0.52)$ & 1.02 & $0.50^{\mathrm{a}}$
\end{tabular}

\begin{tabular}{ccccc} 
Day 7 & $8: 00$ & $22: 00$ & & p-value \\
\hline Dose $(\mathrm{mg})$ & $500(500-720)$ & $550(400-640)$ & n.a. & $0.39^{\mathrm{b}}$ \\
Cmax $(\mathrm{mg} / \mathrm{L})$ & $32.3(3.6)$ & $33.4(3.2)$ & 0.97 & $0.68^{\mathrm{a}}$ \\
AUC $(\mathrm{mg} * \mathrm{~h} / \mathrm{L})^{\mathrm{c}}$ & $101(32)$ & $88(26)$ & 1.15 & $0.34^{\mathrm{a}}$ \\
Cl_TOB $(\mathrm{mL} / \mathrm{min} / \mathrm{kg})$ & $1.42(0.52)$ & $1.54(0.63)$ & 0.92 & $0.87^{\mathrm{a}}$ \\
Vd $(\mathrm{L} / \mathrm{kg})$ & $0.25(0.09)$ & $0.27(0.11)$ & 0.93 & $0.19^{\mathrm{a}}$
\end{tabular}

Dosing and pharmacokinetic parameters are displayed as means or medians with standard deviations or ranges between parentheses, respectively. Abbreviations are: $\mathrm{Cl} \_\mathrm{TOB}$, clearance of tobramycin; Vd, volume of distribution and AUC, area-under-thecurve.

a Student's T-test

b Mann-Whitney test

c Normalized to a tobramycin dose of $500 \mathrm{mg}$ 


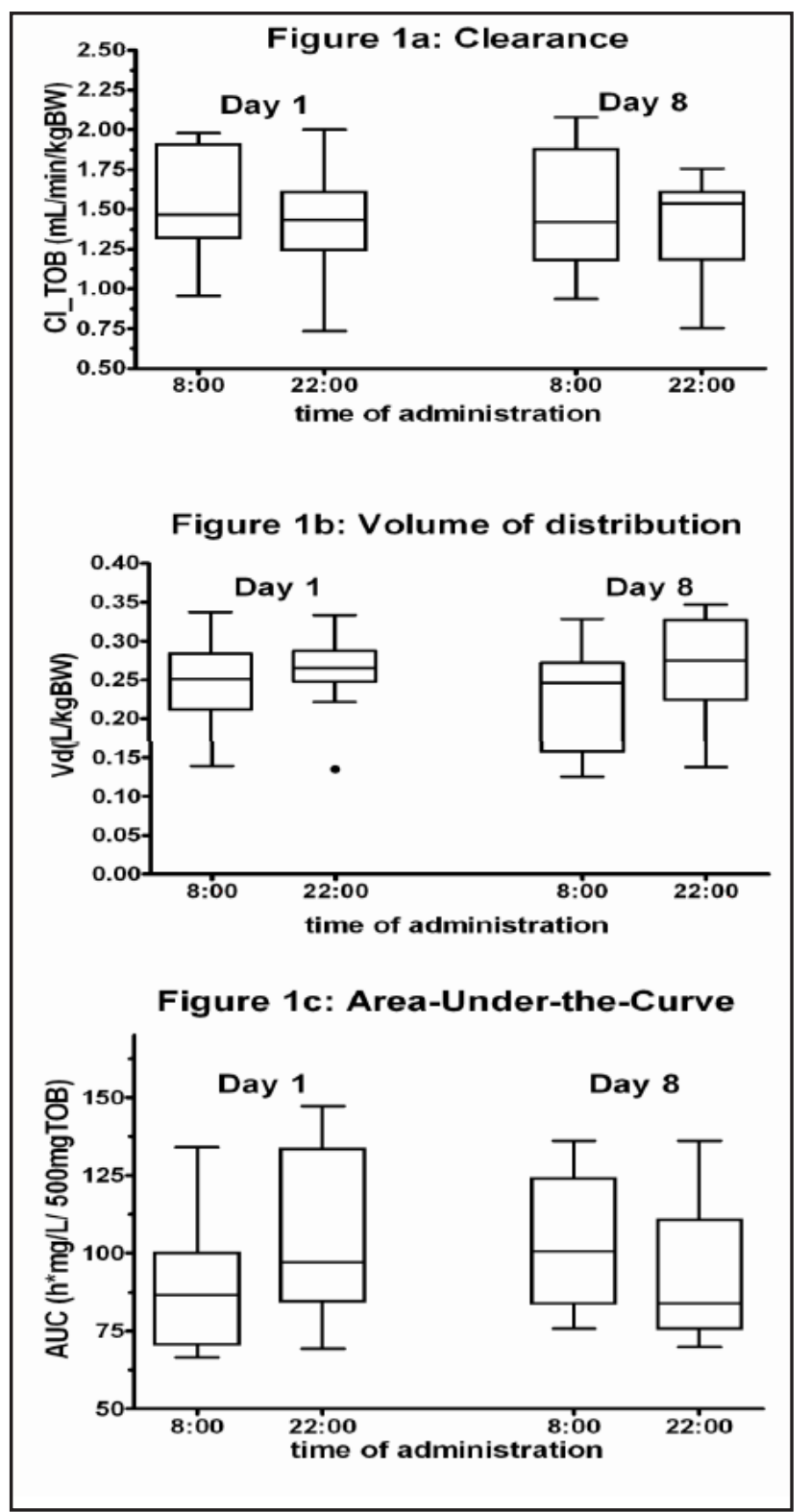

The graphs are box-and-whisker plots of clearance of tobramycin (Cl_TOB, fig 1A), volume of distribution (Vd, fig 1B) and area-under-the-curve (AUC, fig 1C). The line in the middle of each box represents the median; the box extends from the 25th to 75th percentile (interquartile range); and whiskers extend to 1.5 times the interquartile range, rolled back to where data are present. 


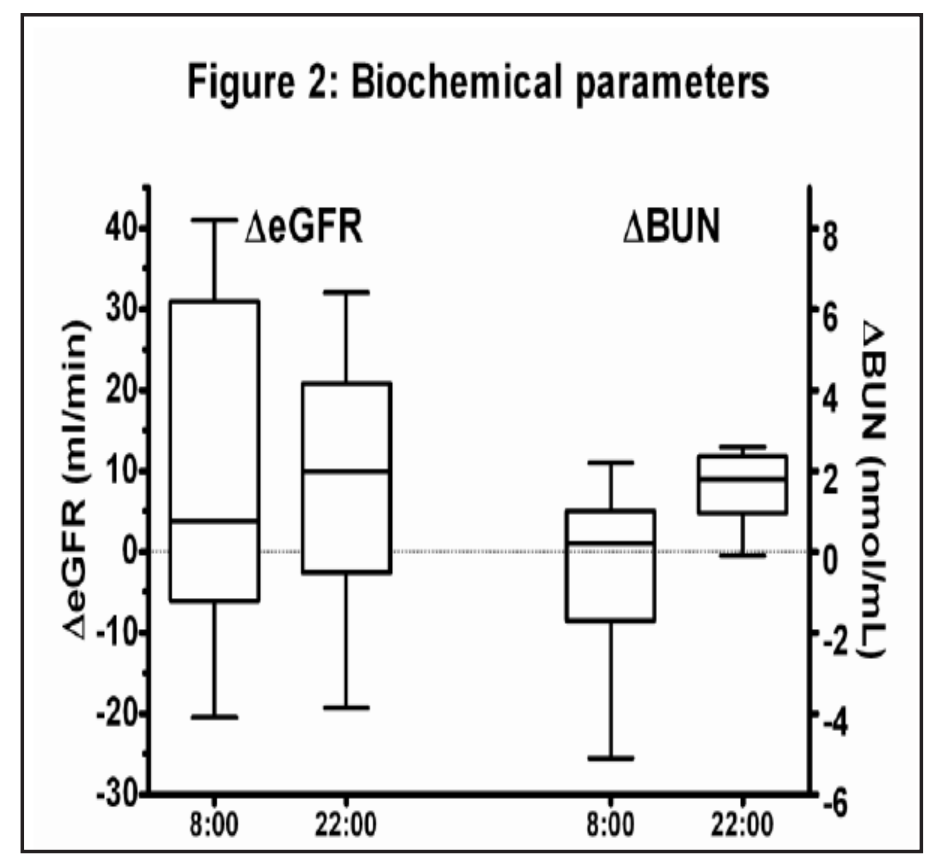

The graph is box-and-whisker plot of the glomerular filtration rate (eGFR) and serum blood urea nitrogen (BUN). The line in the middle of each box represents the median; the box extends from the 25th to 75 th percentile (interquartile range); and whiskers extend to 1.5 times the interquartile range, rolled back to where data are present.

\section{Discussion}

No influence of the time period of administration on tobramycin pharmacokinetics in a hospitalized adult CF population was found in this study. However, we found a rise in serum BUN after one week of therapy in the 22:00 group which is statistically significant compared to the 8:00 group and deserves further exploration.

Since at our clinic only patients in a clinically poor condition suffering from a pulmonary exacerbation are admitted to hospital, the majority is treated at home. Perhaps, the circadian rhythm in ambulatory treated CF populations is preserved during a pulmonary exacerbation. This may select patients who have a less pronounced circadian rhythm as a result of an altered status of the immune system (23). In fact, Vinks et al. found that early-morning serum concentrations of ceftazidime were significantly higher than the afternoon levels in CF patients who were 
treated at home with a continuous infusion (24). Unfortunately, the presence or absence of a normal circadian rhythm was not assessed by measuring melatonin levels for instance. Finally, the contribution of the effect of the circadian may be limited relative to the effect in non-CF patients, since renal clearance of tobramycin is already very rapid in CF patients compared to non-CF patients (18).

This is the first study to examine the effect of diurnal changes on the pharmacokinetics of aminoglycosides in adult CF patients. The pharmacokinetic results were not in line with the results from the posthoc analysis of the TOPIC study (16) and were unexpected as 3 out of 5 studies in hospitalized patients indicate there is an influence of the circadian rhythm on renal clearance of aminoglycosides (11-13). However, the previously mentioned three positive studies were performed in an era where aminoglycosides were multiple daily or even continuously dosed and investigated amikacin, kanamycin and gentamicin that are more likely to accumulate compared to tobramycin $(7,25)$. Furthermore, with an actual aminoglycoside blood concentration of $0.5 \mathrm{mg} / \mathrm{mL}$, most assays report a value somewhere between 0 and $1.0 \mathrm{mg} / \mathrm{mL}$. Use of an inaccurate value may have major consequences for the calculation of clearance. For optimal estimation of tobramycin pharmacokinetics a peak level and a second level at 1.44 half-lives after the end of the administration should be drawn (i.e. between 1.5-9 $\mathrm{h}$ for an adult $\mathrm{CF}$ patient) (26).

In spite of lack of an observed effect of the time of administration on the pharmacokinetics of tobramycin, we found a significant rise in serum BUN in the 22:00 group compared to the 8:00 group. Although a rise in serum BUN of $1.8 \mathrm{nmol} / \mathrm{mL}$ may not be considered clinically relevant, it is rather unexpected since eGFR and exposure in terms of AUC were similar in the 8:00 and 22:00 groups. Perhaps, the former is caused by a disturbance of the diurnal pattern of serum BUN by the nightly exposure to tobramycin, resulting serum BUN to peak in the morning instead of in the evening (27). Furthermore, polycationic aminoglycosides, like tobramycin, can alter ultrastructure and glomerular permeability, tubular reabsorption and intracellular digestion of proteins (27). Finally, a 'false' rise in serum BUN or any other biomarker for that matter caused by interference of tobramycin with the analytical assay of BUN should not be overlooked. Regarding the latter, no effect was detected of tobramycin on BUN concentrations (data not shown). As a result of the known circadian rhythm of biochemical parameters, ideally sampling of these parameters should be performed at several 
time points during the day or as a $24 \mathrm{~h}$-urine collection. Unfortunately, 24h-urine sampling failed in too many patients in our study to perform a statistical analysis of change in mGFR. Future studies addressing this issue should preferably investigate longer periods of exposure to tobramycin (28), and focus on obtaining highly sensitive as well as thoroughly characterized biomarkers for renal toxicity, such as cystatin $\mathrm{C}$, $\beta$-2-microglobulin and $\mathrm{N}$-acetyl- $\beta$-D-glucosaminidase $(29,30)$.

\section{Acknowledgements}

The authors are grateful to Prof $\mathrm{Dr}$ J.H. Proost from the department of Pharmacokinetics, Universitity of Groningen, The Netherlands for his help with the pharmacokinetic analysis. Furthermore, we would like to thank Prof Dr A. Smyth, Department of Child Health, School of Clinical Sciences \& Nottingham Respiratory BRU, University of Nottingham, United Kingdom for his comments on an earlier version of the manuscript.

\section{Declaration of interest statement}

The authors report no conflicts of interest. 


\section{References}

1. Tiddens HA. Detecting early structural lung damage in cystic fibrosis. Pediatr Pulmonol 2002;34(3):228-31.

2. Emerson J, Rosenfeld M, McNamara S, Ramsey B, Gibson RL. Pseudomonas aeruginosa and other predictors of mortality and morbidity in young children with cystic fibrosis. Pediatr Pulmonol 2002;34(2):91-100.

3. Tan KH-V, Hyman-Taylor P, Mulheran M, Knox A, Smyth A. Lack of concordance in the use and monitoring of intravenous aminoglycosides in UK cystic fibrosis centres. Pediatr Pulmonol 2002;32(2):165.

4. Munckhof WJ, Grayson ML, Turnidge JD. A meta-analysis of studies on the safety and efficacy of aminoglycosides given either once daily or as divided doses. $J$ Antimicrob Chemother 1996(37):4.

5. Scheenstra RJ RE, Tavy DL, Kingma H, Heijerman HG. Vestibulotoxicity as a consequence of systemically administered tobramycin in cystic fibrosis patients. Acta Otolaryngol. 2009;129(1):4-7.

6. Prayle A, Smyth AR. Aminoglycoside use in cystic fibrosis: therapeutic strategies and toxicity. Curr Opin Pulm Med. 2010;16(6):604-10.

7. Smyth A, Tan KH-V, Hyman-Taylor P, et al. Once versus three-times daily regimens of tobramycin treatment for pulmonary exacerbations in cystic fibrosis - the TOPIC study, a randomised controlled trial. Lancet 2005;365(9459):573-8.

8. Nakano S, Song J, Ogawa N. Chronopharmacokinetits of gentamicin: comparison between man and mice. Ann Rev Chronopharmacol. Ann Rev Chronopharmacol 1990;7:277-80.

9. Yoshiyama Y, Grenier L, Gourde P, Simard M, Lin L, Morin NJ. Temporal variation in nephrotoxicity of low doses of isepamicin in rats. Antimicrob Agents Chemother 1996;40:802-6.

10. Dickson CJ, Schwartzman MS, Bertino JS. Factors affecting aminoglycoside disposition: effects of circadian rhythm and dietary protein intake on gentamicin pharmacokinetics. Clin Pharmacol Ther 1986;39:325-8.

11. Lucht F, Tigaud S, Esposito G, et al. Chronokinetic study of netilmicin in man. Eur J Clin Pharmacol 1990;39:199-201.

12. Bleyzac N, Allard-Latour B, Laffont A, et al. Diurnal changes in the pharmacokinetic behavior of amikacin. Ther Drug Monit 2000;22:307-12.

13. Elting L, Bodey GP, Rosenbaum B, Fainstein V. Circadian variation in serum amikacin levels. J Clin Pharmacol 1990;30:798-801.

14. Touw DJ, Vinks AA, Heijerman HG, Hermans J, Bakker W. Suggestions for the optimization of the initial tobramycin dose in adolescent and adult patients with cystic fibrosis. Ther Drug Monit. 1994;16(2):125-31.

15. Beauchamp D, Labrecque G. Chronobiology and chronotoxicology of antibiotics and aminoglycosides. Adv Drug Deliv Rev. 2007;59(9-10):896-903.

16. Touw DJ, Knox AJ, Smyth A. Population pharmacokinetics of tobramycin administered thrice daily and once daily in children and adults with cystic fybrosis. Journal of Cystic Fibrosis 2007;6(5):327-33.

17. Fuchs HJ, Borowitz DS, Christiansen DH, et al. Effect of aerosolised recombinant human DNase on exacerbations of respiratory symptoms and on pulmonary function in patients with cystic fibros. N Engl J Med 1994;331:637-42.

18. Touw DJ, Vinks AA, Neef C. Pharmacokinetic modelling of intravenous tobramycin in adolescent and adult patients with cystic fibrosis using the nonparametric 
expectation maximization (NPEM) algorithm. Pharm World Sci. 1997;19(3):142-51.

19. Proost JH, Meijer DK. MW/Pharm, an integrated software package for drug dosage regimen calculation and therapeutic drug monitoring. Comput Biol Med. 1992;22(3):155-63.

20. Jelliffe RW. Estimation of creatinine clearance when urine cannot be collected. Lancet 1971;975-6 i.

21. Cano EL, Haque NZ, Welch VL, Cely CM, Peyrani P, Scerpella EG, et al. Incidence of nephrotoxicity and association with vancomycin use in intensive care unit patients with pneumonia: retrospective analysis of the IMPACT-HAP Database. Clinical therapeutics;34(1):149.

22. Bellomo R, Ronco C, Kellum JA, Mehta RL, Palevsky P, Acute Dialysis Quality Initiative w. Acute renal failure - definition, outcome measures, animal models, fluid therapy and information technology needs: the Second International Consensus Conference of the Acute Dialysis Quality Initiative (ADQI) Group. Critical Care (London, England) 2004;8(4):R204.

23. Mundigler G, Delle-Karth G, Koreny M, et al. Impaired circadian rhythm of melatonin secretion in sedated critically ill patients with severe sepsis. Crit Care Med 2002;30:536-540.

24. Vinks AA TD, Heijerman HG, Danhof M, de Leede GP, Bakker W. Pharmacokinetics of ceftazidime in adult cystic fibrosis patients during continuous infusion and ambulatory treatment at home. Ther Drug Monit. 1994;16(4):341-8.

25. De Broe ME GR, Verpooten GA. Choice of drug and dosage regimen. Two important risk factors for aminoglycoside nephrotoxicity. Am J Med. 1986;80(6b):115-8. 26. Jelliffe RW, Iglesias T, Hurst AK, et al. Individualising gentamicin dosage regimens: a comparative review of selected models, data fitting methods and monitoring strategies. Clin Pharmacokinet 1991;21:461-78.

27. Singh RK, Singh Y, Srivastava R. Circadian variation of serum urea concentration in north Indian subjects. Indian J Med Res. 1990;92:257-9.

28. Croes S KA, van Gils SA, Neef C. Efficacy, nephrotoxicity and ototoxicity of aminoglycosides, mathematically modelled for modelling-supported therapeutic drug monitoring. Eur J Pharm Sci. 2012;45(1-2):90-100.

29. Prayle A, Watson A, Fortnum H, Smyth A. Side effects of aminoglycosides on the kidney, ear and balance in cystic fibrosis. Thorax. 2010;65(7):654-8.

30. Inker LA, Schmid $\mathrm{CH}$, Tighiouart $\mathrm{H}$,et al. Estimating Glomerular Filtration Rate from Serum Creatinine and Cystatin C. N Engl J Med 2012;367:20-9. 


\title{
Chapter 2.2
}

\section{Chronopharmacokinetics of Once Daily Dosed Aminoglycosides in Clinically Infected Patients}

\author{
ERIK VAN MAARSEVEEN \\ WAI HONG MAN \\ JOHANNES PROOST \\ CEES NEEF \\ DANIËL TOUW
}

under revision
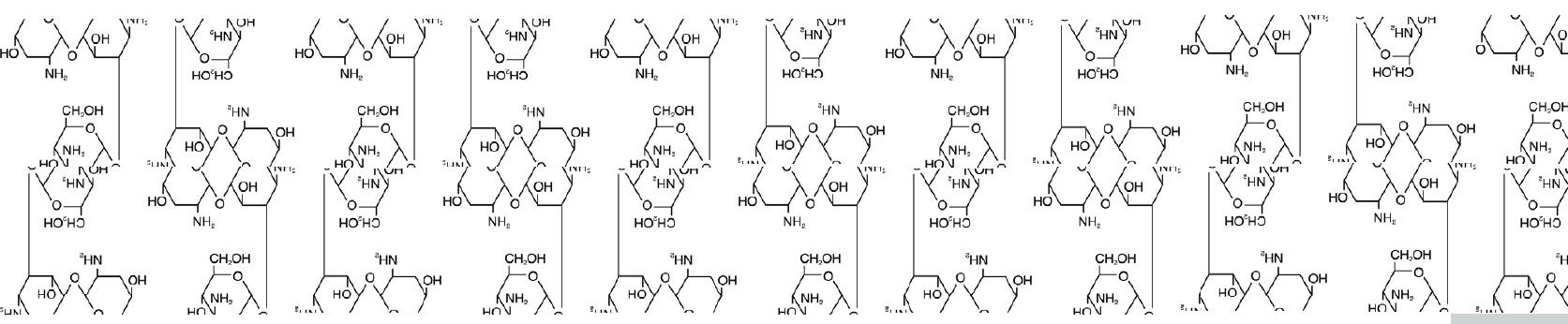


\section{Abstract}

Clinically infected patients treated with aminoglycosides are at risk of developing nephrotoxicity. It has been suggested that the pharmacokinetics of aminoglycosides in humans follows a circadian rhythm. Therefore, the time period of administration could have important clinical implications in once daily dosing regimens primarily with regard to the incidence of nephrotoxicity. In this retrospective cohort study, the effect of the clock time of administration on aminoglycoside exposure and the incidence of nephrotoxicity in a large population of clinically infected patients was investigated. Patients treated with intravenous tobramycin or gentamicin were eligible for inclusion. Patients were divided into three groups by time of administration: morning, afternoon and night. Pharmacokinetic parameters and the incidences of nephrotoxicity were compared between the morning, afternoon and evening groups. 310 general ward and 411 intensive care unit patients were included. No significant differences were found in patient characteristics between the morning, afternoon and night groups. The time period of administration did not affect aminoglycoside exposure $(p=0.97)$ or the incidence of nephrotoxicity $(p=0.47)$. This large retrospective study shows that the time period of administration had no effect on the pharmacokinetics or nephrotoxicity of ODD aminoglycosides in hospitalized patients. Consequently, we advise aminoglycosides to be administered as soon as possible in case of (suspected) severe hospital-acquired infections and subsequent dosages to be based on therapeutic drug monitoring.

\section{Introduction}

Aminoglycoside antibiotics remain an important group of antimicrobial agents in the treatment of serious infections by gram-negative organisms (1). Although they are highly effective in killing these organisms, exposure to intravenous aminoglycosides comes with an increased risk of auditory and renal toxicity (2). All hospitalized patients, especially critically ill patients with a low renal clearance of aminoglycosides are at high risk of developing nephrotoxicity $(3,4)$. In an attempt to reduce the nephrotoxic side effects without compromising efficacy, once-daily dosing regimens of aminoglycosides have shown to be superior to conventional multiple daily dosing regimens (5). 
Nowadays, intravenous aminoglycosides are mainly dosed once daily worldwide (6). Since a circadian rhythm of aminoglycoside pharmacokinetics has been observed in animal experiments (7-11) as well as in human studies involving multiple daily dosing protocols $(8,12,13)$, the clearance of aminoglycosides is expected to be higher in the active period versus the resting period caused by an increase in glomerular filtration rate. As a result, the time of administration in patients receiving their drug with 24-hour intervals could have important implications with regards to aminoglycoside clearance and thus exposure. Nevertheless, human data involving the effect of the circadian rhythm on pharmacokinetics and toxicity of once daily dosed (ODD) aminoglycosides are inconsistent $(14,15)$. To date, the effect of diurnal changes on key pharmacokinetic parameters of aminoglycoside exposure has never been extensively studied in a large cohort of patients with serious infections.

\section{Aim of the study}

To examine the effect of time period of administration on aminoglycoside exposure and the incidence of nephrotoxicity in a large population of clinically infected patients.

\section{Ethical approval}

A waiver for consent was provided by the local medical ethics committee.

\section{Methods}

In this retrospective cohort study data were extracted from patients' clinical records and pharmacokinetic database. All general ward and intensive care unit patients of 18 years or older who were admitted hospital between January 2006 and December 2013 and were treated with intravenous tobramycin or gentamicin were included. Furthermore, an estimated glomerular filtration rate calculated with the MDRD formula (16) higher than $25 \mathrm{~mL} / \mathrm{min}$ and a once daily dose of 4-6 mg/kg were required. Finally, two tobramycin concentrations drawn after the first or second infusion were mandatory for inclusion. For the analysis of nephrotoxicity in the general ward population, the treatment duration had to be two days or longer. Furthermore, a minimum of two serum 
creatinine concentrations was required; one drawn between 48 hours before and 12 hours after initiation of therapy. If two or more concentrations were available during this time window the concentration drawn closest to the time of intiation of aminoglycoside therapy was selected as the baseline creatinine concentration. The second 'post-treatment' concentration was drawn within 48 hours after initiation and 48 hours after cessation of therapy was mandatory for inclusion. If two 'post-treatment' were available in this period the highest creatinine concentration was appointed as the 'post-treatment' concentration. The delta serum creatinine $(\Delta \mathrm{Scr})$ was derived by subtracting the 'post-treatment' with the baseline creatinine. Nephrotoxicity was defined as a $\Delta \mathrm{Scr}$ of at least $45 \mathrm{nmol} / \mathrm{mL}(0.5 \mathrm{mg} / \mathrm{dL})$ from baseline (15). For the analysis of nephrotoxicity in the general ward population, a minimal treatment duration of 2 days was required for inclusion. According to the monitoring protocol, a peak concentration and second concentration were drawn $0.5-1 \mathrm{~h}$ and 6-14 $\mathrm{h}$ after end of the first or second infusion, respectively. Aminoglycoside dosing was guided by drug concentration monitoring targeting peak concentrations of $15-20 \mathrm{mg} / \mathrm{L}$, and estimated trough concentrations $<0.5 \mathrm{mg} / \mathrm{L}$. It was a priori decided to analyze the pharmacokinetics separately for general ward and intensive care unit populations and to analyze nephrotoxicity only in the general ward population because the kidney function of critically ill patients admitted to the intensive care unit has an inherently large variability and many artifacts occur due to cardiovascular management (17). Patients were divided into three groups by time of administration: 5:00-12:55 (morning), 13:00-20:55 (afternoon) and 21:00-4:55 (night). Pharmacokinetic parameters were calculated using pharmacokinetic software (MWI Pharm 3.80, MediWare, Zuidhoorn, The Netherlands) and population pharmacokinetic models (18).

\section{Statistical analysis}

A Kolmogorov-Smirnov test was used to examine normal distribution of the data. Means and standard deviations of continuous variables are presented for normal distributions. Otherwise, medians and ranges were reported. One-way ANOVA with bonferroni post hoc tests were used to determine statistical differences among normally distributed groups. If data were not normally distributed, a Kruskal-Wallis non-parametric method was applied with Mann-Whitney-U post hoc tests 
including correction for multiple testing. A Chi-square test was used to compare dichotomous variables between groups. All Statistical analysis were performed using GraphPad Prism version 5.03 for Windows (GraphPad Software, San Diego, CA, USA). A p-value of $<0.05$ was considered to be statistically significant and in each instance a 2-sided test was carried out.

\section{Results}

A total of 1452 general ward and 843 intensive care unit patients were selected from the database. Approximately half of the selected general ward and intensive care unit patients were excluded as a result of missing pharmacokinetic data. In the general ward population, subsequently again half of the remaining patients were excluded from the nephrotoxicity analysis because of missing creatinine values or a treatment duration shorter than two days. 310 general ward and 411 intensive care unit patients were eligible for inclusion in the morning, afternoon and night groups. No significant differences were found in patient characteristics between the three groups within both populations (Table 1).

Although a trend toward a higher volume of distribution in the afternoon group was seen in the general ward population compared to the morning or night groups $(0.28,0.25$ and $0.24 \mathrm{~L} / \mathrm{kg}$ respectively, $p=0.06$ ), tobramycin clearance and area-under-the-curve did not differ significantly between the morning, afternoon and night groups in both populations (Table 2). Finally, no significant differences in delta serum creatinine $(p=0.11)$ or in percentages of nephrotoxicity $(p=0.47)$ were found between the morning, afternoon and night groups of the general ward population (Table 2). 
Table 1. Patient characteristics

\begin{tabular}{|c|c|c|c|c|}
\hline \multicolumn{5}{|c|}{ Time of administration } \\
\hline general ward & $\begin{array}{c}\text { Morning } \\
(\mathrm{n}=88)\end{array}$ & $\begin{array}{c}\text { Afternoon } \\
(\mathrm{n}=108)\end{array}$ & $\begin{array}{c}\text { Night } \\
(\mathrm{n}=114)\end{array}$ & $\mathrm{p}$-value \\
\hline age (years) & $63(34)$ & $62(27)$ & $57(31)$ & $0.56^{\mathrm{a}}$ \\
\hline weight $(\mathrm{kg})$ & $70(29)$ & $71(27)$ & $70(26)$ & $0.33^{\mathrm{a}}$ \\
\hline$\%$ of males & 42 & 48 & 56 & $0.10^{\mathrm{c}}$ \\
\hline$\%$ of patients on TOB & 21 & 27 & 19 & $0.23^{c}$ \\
\hline eGFR (ml/min) & $80(26-191)$ & $88(26-189)$ & $89(26-185)$ & $0.27^{\mathrm{b}}$ \\
\hline intensive care unit & $\begin{array}{l}\text { Morning } \\
(\mathrm{n}=124)\end{array}$ & $\begin{array}{c}\text { Afternoon } \\
(\mathrm{n}=145)\end{array}$ & $\begin{array}{c}\text { Night } \\
(\mathrm{n}=142)\end{array}$ & $\mathrm{p}$-value \\
\hline age (years) & $68(32)$ & $65(28)$ & $68(31)$ & $0.14^{\mathrm{a}}$ \\
\hline weight $(\mathrm{kg})$ & $72(28)$ & $75(26)$ & $75(24)$ & $0.48^{\mathrm{a}}$ \\
\hline$\%$ of males & 64 & 65 & 69 & $0.47^{\mathrm{c}}$ \\
\hline$\%$ of patients on TOB & 48 & 43 & 39 & $0.35^{\mathrm{c}}$ \\
\hline eGFR (ml/min) & $55(25-167)$ & $64(25-183)$ & $57(25-167)$ & $0.41^{\mathrm{b}}$ \\
\hline
\end{tabular}

Dichotomous variables are displayed as percentages. Continuous variables are presented as means \pm standard deviations or medians with ranges in parentheses (minmax). Abbreviations are: eGFR, estimated glomular filtration rate; SAPS, simplified acute physiology score; TOB, tobramycin.

a ANOVA

b Kruskall-Wallis test

c Chi-square test 
Table 2. Dosing and pharmacokinetic parameters

\begin{tabular}{|c|c|c|c|c|}
\hline General Ward & $\begin{array}{l}\text { Morning } \\
(\mathrm{n}=88)\end{array}$ & $\begin{array}{c}\text { Afternoon } \\
(\mathrm{n}=108)\end{array}$ & $\begin{array}{l}\text { Night } \\
(\mathrm{n}=114)\end{array}$ & p-value \\
\hline Dose (mg) & $350(180-500)$ & $360(220-600)$ & $350(240-650)$ & $0.48^{\mathrm{b}}$ \\
\hline Treatment duration (days) & $5(3-18)$ & $5(3-22)$ & $5(3-21)$ & $0.98^{\mathrm{b}}$ \\
\hline $\mathrm{CL}(\mathrm{L} / \mathrm{h})$ & $4.4(1.7)$ & $4.6(1.8)$ & $4.7(1.7)$ & $0.41^{\mathrm{a}}$ \\
\hline $\mathrm{Vd}(\mathrm{L} / \mathrm{kg})$ & $0.25(0.08)$ & $0.28(0.09)$ & $0.24(0.08)$ & $0.06^{\mathrm{a}}$ \\
\hline $\mathrm{AUC}(\mathrm{mg} * \mathrm{~h} / \mathrm{L})$ & $135(47)$ & $139(48)$ & $145(51)$ & $0.97^{\mathrm{a}}$ \\
\hline Delta Scr $(\mathrm{nmol} / \mathrm{mL})$ & $6(-40-167)$ & $0(-11-323)$ & $-1(-56-467)$ & $0.11^{\mathrm{b}}$ \\
\hline Nephrotoxicity (\%) & 18 & 12 & 10 & $0.47^{\mathrm{c}}$ \\
\hline ICU & $\begin{array}{l}\text { Morning } \\
(\mathrm{n}=124)\end{array}$ & $\begin{array}{c}\text { Afternoon } \\
(\mathrm{n}=145)\end{array}$ & $\begin{array}{c}\text { Night } \\
(n=142)\end{array}$ & p-value \\
\hline Dose (mg) & $350(250-675)$ & $360(240-600)$ & $360(150-750)$ & $0.36^{\mathrm{b}}$ \\
\hline Treatment duration (days) & $4(3-12)$ & $4(3-13)$ & $5(3-17)$ & $0.72^{\mathrm{b}}$ \\
\hline $\mathrm{CL}(\mathrm{L} / \mathrm{h})$ & $1.9(0.06)$ & $2.1(0.08)$ & $2.1(0.07)$ & $0.19^{\mathrm{a}}$ \\
\hline $\mathrm{Vd}(\mathrm{L} / \mathrm{kg})$ & $0.33(0.11)$ & $0.32(0.10)$ & $0.33(0.12)$ & $0.85^{\mathrm{a}}$ \\
\hline AUC (mg*h/L) & $195(71)$ & $183(75)$ & $173(67)$ & $0.86^{\mathrm{a}}$ \\
\hline
\end{tabular}

Continuous variables are presented as

means \pm standard deviations or medians with ranges in parentheses (min-max). Abbreviations are: eGFR, estimated glomular filtration rate; AUC, area under the concentration time curve; $\mathrm{CL}$, clearance; $\mathrm{Scr}$, serum creatinine; $\mathrm{Vd}$, volume of distribution, $\Delta \mathrm{Scr}$, median delta serum creatinine concentration

a ANOVA

b Kruskall-Wallis test

c Chi-square test 


\section{Discussion}

Since most previous studies did find an effect of the circadian rhythm on the pharmacokinetics of aminoglycosides, our results intuitively seem unexpected. Nevertheless, an in-depth review of the available evidence demonstrates that most studies were performed in relatively small populations of animals or (young) healthy volunteers, primarily investigated multiple daily or even continuously dosed aminoglycosides, and had suboptimal pharmacokinetic sampling protocols with only peak and trough concentration monitoring and/or reported on amikacin and kanamycin that are more likely to accumulate (7-13, 19-22). In addition and perhaps most eminent, therapeutic drug monitoring in the majority of studies was performed after 3 days of therapy at 'steady state'. At that moment in time the signs of clinical infection will have resolved in a large proportion of patients and aminoglycosides are then withdrawn in current clinical practice. It is thus critical to collect pharmacokinetic data during the first two days of therapy when the patient still shows signs of a clinical infection, since sepsis and an altered status of the immune system disrupt the circadian rhythm $(23,24)$. Our study investigated a large population of clinically infected patients, who received their aminoglycosides ODD with optimal pharmacokinetic sampling during the first or the second administration.

Caution needs to be exercised when interpreting the results of any retrospective cohort study because of errors due to confounding and selection bias. However, baseline patient characteristics showed no significant differences between groups and the proportion of excluded patients per exclusion criterion did not differ significantly between patients who were administered aminoglycosides in the morning, afternoon and night (data not shown). Furthermore, coadministration of nephrotoxic medication was not included in this analysis, but a correlation between the use of e.g. furosemide or other nephrotoxic antibiotics and the time of administration of aminoglycosides is highly unlikely. As mentioned, the results obtained from studies examining the effect of the circadian rhythm on the pharmacokinetics of aminoglycosides in patient populations are conflicting $(15,25-28)$, but can be explained by the same conveyable causes for the vast majority. The three 'positive' studies all involved multiple daily dosing protocols of 'earlier', more accumulating aminoglycosides (e.g. kanamycin and amikacin). In contrast, the two 'negative' studies reported on once or twice daily dosed 
aminoglycosides that show relatively low tissue accumulation (e.g. tobramycin, netelmicin and gentamicin). A randomized controlled trial by Fauvalle et al., in which netilmicin $(4.5 \mathrm{mg} / \mathrm{kg}$ of lean body weight) was administered every $24 \mathrm{~h}$ at 10 a.m. or 10 p.m. to 23 ill febrile patients with severe infection, showed that the time of administration had no influence on netilmicin concentrations in serum nor on pharmacokinetic parameters in these patients (27). The second was a prospective cohort study by Prins et al., who investigated the influence of drug administration time on serum drug concentrations and the incidence of nephrotoxicity in 179 patients with serious infections treated with gentamicin or tobramycin once daily. The authors did not find statistically significant differences in trough or peak concentrations between the time periods of administration. Nevertheless, the incidence of nephrotoxicity was significantly higher when the aminoglycosides were administered to a combined general ward and intensive care unit population during the resting period (midnight to $7: 30 \mathrm{AM} ; \mathrm{p}=0.004$ ). In our opinion, data on pharmacokinetics and nephrotoxicity of aminoglycosides in general ward and intensive care unit populations should be analyzed separately since admittance to the intensive care unit is a known risk factor for acute kidney injury $(29,30)$. Moreover, the study by Prins et al. demonstrated that intensive care unit patients are more 'at risk' to receive their aminoglycosides during the night (77 vs. $44 \%$ ) (15). Admittance to the intensive care unit is thereby a confounder by definition, since it correlates with both the dependent (i.e. nephrotoxicity) and the independent variable (i.e. time of administration). Furthermore, the authors had to statistically correct for not only the higher prevalance of intensive care unit admittance but also for significantly poorer baseline creatinine clearance (84 vs. $100 \mathrm{ml} / \mathrm{min}, \mathrm{p}<0.05$ ) in the resting compared to the active period. All of the above are established risk factors of acute kidney injury $(29,30)$. Altogether, it is debatable whether statistical correction is a proper tool to adjust for these imbalances in patient characteristics.

Our data show a trend of a higher volume of distribution in the afternoon group of the general ward population but not in the intensive care unit population. This is probably caused by the discrepancy in the daily fluid management of a general ward patient compared to an intensive care unit patient. Whereas the latter will receive intravenous fluids continuously contributing to a relatively stable volume of distribution, a general ward patient will gain fluids during the day causing the volume of distribution of hydrophilic aminoglycosides to peak in the afternoon. 
The presented pharmacokinetic data are in accordance with the data reported by Fauvalle et al.(27) and are further supported by the unpublished results of two recently performed randomized clinical trials in adult and pediatric cystic fibrosis patients suffering from pulmonary exacerbation that also found no effect of the circadian rhythm on the pharmacokinetics of once daily dosed tobramycin in cystic fibrosis patients (submitted for publication).

\section{Conclusion}

Currently it is widely believed that administration of aminoglycosides in the morning will result in a reduction in nephrotoxicity and many (international) antibiotic guidelines advice to administer aminoglycosides in the morning. This large retrospective study, however, shows no administration-time-related differences in aminoglycoside exposure or nephrotoxicity in clinically infected patients. It is therefore unlikely that administration during the resting period will result in a higher incidence of renal toxicity in these patients. We recommend aminoglycosides to be administered as soon as possible in case of (suspected) severe hospital-acquired pneumonia or sepsis to reduce the pen-to-needle time in antibiotics, which has shown to be effective in preventing death (31). Subsequent dosages should be based on therapeutic drug monitoring to optimize the efficacy/toxicity balance.

\section{Funding}

No funding was received for this study.

\section{Competing interests}

The authors declare that they have no competing interests. 


\section{References}

1. Beaucaire G, Leroy O, Beuscart C, Karp P, Chidiac C, Caillaux M. Clinical and bacteriological efficacy, and practical aspects of amikacin given once daily for severe infections. J Antimicrob Chemother 1991;27 Suppl C:91-103. 2. Munckhof WJ, Grayson ML, Turnidge JD. A meta-analysis of studies on the safety and efficacy of aminoglycosides given either once daily or as divided doses. J Antimicrob Chemother 1996(37):4.

3. Buijk SE, Mouton JW, Gyssens IC, Verbrugh HA, Bruining HA. Experience with a once-daily dosing program of aminoglycosides in critically ill patients. Intensive Care Med 2002;28(7):936-42.

4. Hansen M, Christrup LL, Jarlov JO, Kampmann JP, Bonde J. Gentamicin dosing in critically ill patients. Acta Anaesthesiol Scand 2001;45(6):73440.

5. Hatala R, Dinh T, Cook DJ. Once-daily aminoglycoside dosing in immunocompetent adults: a meta-analysis. Ann Intern Med 1996;124(8):717-25. 6. Bodmann KF. Current guidelines for the treatment of severe pneumonia and sepsis. Chemotherapy 2005;51(5):227-33.

7. Hosokawa H, Nyu S, Nakamura K, Mifune K, Nakano S. Circadian variation in amikacin clearance and its effects on efficacy and toxicity in mice with and without immunosuppression. Chronobiol Int 1993;10:259-79.

8. Nakano S, Song J, Ogawa N. Chronopharmacokinetits of gentamicin: comparison between man and mice. Ann Rev Chronopharmacol. Ann Rev Chronopharmacol 1990;7:277-80.

9. Song J, Ohdo S, Ogawa N, Nakano S. Influence of feeding schedule on chronopharmacological aspects of gentamicin in mice. Chronobiol Int 1993;10:338-48.

10. Yoshiyama Y, Kobayashi T, Tomonaga F. Chronotoxical study of gentamicin induced nephrotoxicity in rats. J Antibiot (Tokyo) 1992;45:806-8.

11. Yoshiyama Y, Nishikawa S, Sugiyama T, Kobayashi T, Shimada H, Tomonaga F, et al. Influence of circadian-stage-dependent dosing schedule on nephrotoxicity and pharmacokinetics of isepamicin in rats. Antimicrob Agents Chemother 1993;37:2042-3.

12. Dickson CJ, Schwartzman MS, Bertino JS. Factors affecting aminoglycoside disposition: effects of circadian rhythm and dietary protein intake on gentamicin pharmacokinetics. Clin Pharmacol Ther 1986;39:325-8.

13. Yoshiyama Y, Grenier L, Gourde P, Simard M, Lin L, Morin NJ. Temporal variation in nephrotoxicity of low doses of isepamicin in rats. Antimicrob Agents Chemother 1996;40:802-6.

14. Beauchamp D, Labrecque G. Chronobiology and chronotoxicology of antibiotics and aminoglycosides. Adv Drug Deliv Rev. 2007;59(9-10):896-903. 15. Prins JM, Weverling GJ, van Ketel RJ, et al. Circadian variations in serum levels and the renal toxicity of aminoglycosides in patients. Clin Pharmacol Ther 1997;62:106-111. 
16. Levey AS, Bosch JP, Lewis JB, Greene T, Rogers N, Roth D. A more accurate method to estimate glomerular filtration rate from serum creatinine: a new prediction equation. Modification of Diet in Renal Disease Study Group. Ann Intern Med 1999;130(6):461-70.

17. Bellomo R, Ronco C, Kellum JA, Mehta RL, Palevsky P, Acute Dialysis Quality Initiative w. Acute renal failure - definition, outcome measures, animal models, fluid therapy and information technology needs: the Second International Consensus Conference of the Acute Dialysis Quality Initiative (ADQI) Group. Critical Care (London, England) 2004;8(4):R204.

18. Proost JH, Meijer DK. MW/Pharm, an integrated software package for drug dosage regimen calculation and therapeutic drug monitoring. Comput Biol Med. 1992;22(3):155-63.

19. Lin L, Grenier L, Bergeron Y, Simard M, Bergeron MG, Labrecque G, et al. Temporal changes of pharmacokinetics, nephrotoxicity, and subcellular distribution of tobramycin in rats. Antimicrob Agents Chemother 1994;38(1):5460.

20. Jelliffe RW. Estimation of creatinine clearance when urine cannot be collected. Lancet 1971;975-6 i.

21. Fauconneau B, De Lemos E, Pariat C, Bouquet S, Courtois P, Piriou A. Chronotoxicity in rat of a vancomycin and gentamicin combination. Pharmacol Toxicol 1992;71:31-6.

22. Pariat C, Courtois P, Cambar J, Piriou A, Bouquet S. Circadian variations in the renal toxicity of gentamicin in rats. Toxicol Lett 1988;40:175-82.

23. Verceles AC, Silhan L, Terrin M, Netzer G, Shanholtz C, Scharf SM. Circadian rhythm disruption in severe sepsis: the effect of ambient light on urinary 6-sulfatoxymelatonin secretion. Intensive Care Med;38(5):804-10.

24. Habbal OA, Al-Jabri AA. Circadian rhythm and the immune respons: a review. It Rev Immunol 2009;28:93-108.

25. Bleyzac N, Allard-Latour B, Laffont A, et al. Diurnal changes in the pharmacokinetic behavior of amikacin. Ther Drug Monit 2000;22:307-12.

26. Elting L, Bodey GP, Rosenbaum B, Fainstein V. Circadian variation in serum amikacin levels. J Clin Pharmacol 1990;30:798-801.

27. Fauvelle F, Perrin P, Belfayol L, et al. Fever and associated changes in glomerular filtration rate erase anticipated diurnal variations in aminoglycoside pharmacokinetics. Antimicrob Agents Chemother 1994;38:620-3.

28. Lucht F, Tigaud S, Esposito G, et al. Chronokinetic study of netilmicin in man. Eur J Clin Pharmacol 1990;39:199-201.

29. Bertino JS, Jr., Booker LA, Franck PA, Jenkins PL, Franck KR, Nafziger AN. Incidence of and significant risk factors for aminoglycoside-associated nephrotoxicity in patients dosed by using individualized pharmacokinetic monitoring. J Infect Dis 1993;167(1):173-9. 
30. Zappitelli M, Parikh CR, Akcan-Arikan A, Washburn KK, Moffett BS, Goldstein SL. Ascertainment and epidemiology of acute kidney injury varies with definition interpretation. Clin J Am Soc Nephrol 2008;3(4):948-54.

31. Kumar A, Roberts D, Wood KE, Light B, Parrillo JE, Sharma S, et al. Duration of hypotension before initiation of effective antimicrobial therapy is the critical determinant of survival in human septic shock. Crit Care Med 2006;34(6):1589-96. 


\title{
Chapter 2.3
}

\section{Once Daily Dosed Gentamicin is more Nephrotoxic than Once Daily Dosed Tobramycin in Clinically Infected Patients}

\author{
ERIK VAN MAARSEVEEN \\ MARIE-CHRISTINE VAN BUUL-GAST \\ RAHAT ABDOELLAKHAN \\ LUC GELINCK \\ CEES NEEF \\ DANIEL TOUW
}

Adapted from J Antimicrob Chemother. 2014 Sep;69(9):2581-3.

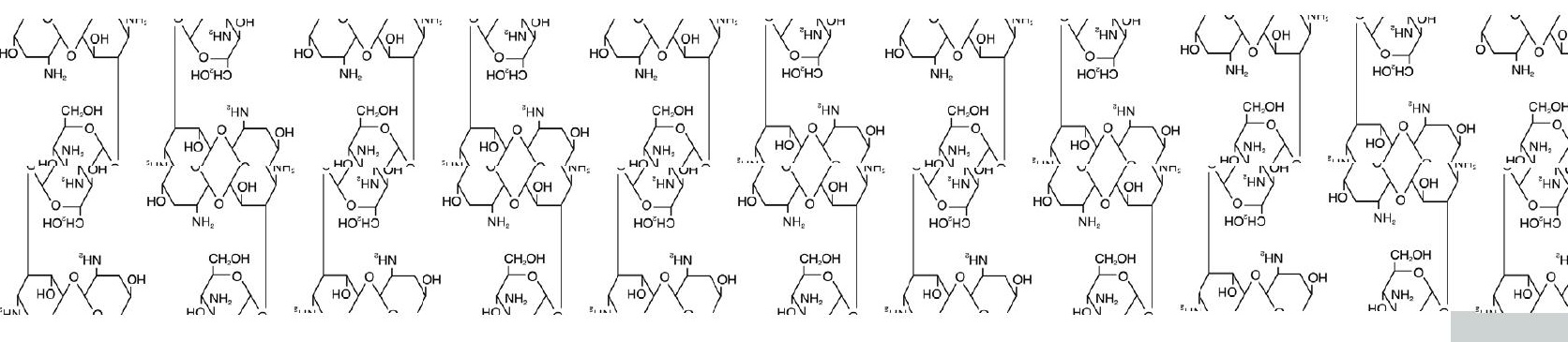




\section{Abstract}

Randomized clinical trials and meta-analyses have shown that multiple daily dosed (MDD) tobramycin is less nephrotoxic than MDD gentamicin. However, no prior studies have compared nephrotoxicity of tobramycin versus gentamicin when administered as nowadays more common once daily dosing (ODD) regimens. Hence, the present study aimed to investigate the nephrotoxicity of ODD gentamicin and tobramycin. A retrospective cohort analysis was performed on data of adult patients, treated with a once daily dose of $5 \mathrm{mg} / \mathrm{kg}$ gentamicin or tobramycin for three days or longer. As local treatment protocol switched from gentamicin to tobramycin in February 2010, gentamicin treated patients were selected from January 2008 to February 2010, while tobramycin treated patients were selected from March 2010 to December 2012. Information on gender, age, kidney function, dose, treatment duration, and aminoglycoside serum concentrations was collected. A multivariate logistic regression analysis was performed to identify covariates predictive of nephrotoxicity. Of the 2889 patients who were eligible, 202 gentamicin and 180 tobramycin met the inclusion criteria and were evaluated. Baseline characteristics did not differ between both groups. Nephrotoxicity developed in 43 of $202(21,2 \%)$ of gentamicin and 19 of $180(10.6 \%)$ of tobramycin treated patients (odds ratio (OR) 2.4, 95\% confidence interval $(95 \% \mathrm{Cl}) 1.2-4.7)$. Furthermore, an area-under-the-curve (AUC) of the first administration $>150 \mathrm{mg}^{*} \mathrm{~h} / \mathrm{L}$ was independently predictive of developing nephrotoxicity (OR $2.2,95 \% \mathrm{Cl}$ 1.1-5.0). ODD tobramycin was less nephrotoxic than ODD gentamicin. Moreover, early AUC monitoring seems warranted to identify patients at high risk of developing nephrotoxicity.

\section{Introduction}

During the late 1970s, the commercial launch of broadspectrum antibiotics with fewer side effects, such as fluoroquinolones, carbapenems, cephalosporins, and $\beta$-lactam/ $\beta$-lactamase inhibitor combinations, led to a declined clinical application of aminoglycosides (1). Unfortunately, over the past forty years, a number of resistance mechanisms have emerged in gram-negative pathogens that pose a serious threat to our common antibiotic armamentarium. 
As the pharmaceutical pipeline wanes drugs that are active against multi-drug resistant gram-negative organisms aminoglycosides have regained interest (2).

Aminoglycosides are very potent bactericidal antimicrobals, but nephrotoxicity is an important factor limiting their clinical utility. Although this is a clinically relevant adverse drug event of all systemic aminoglycosides, the nephrotoxic potential appears to differ within the drug class. For instance, compared to tobramycin gentamicin has a relatively high tissue accumulation in the renal proximal tubules, explaining its increased nephrotoxic potential (3-8). Furthermore, animal studies have confirmed gentamicin is more nephrotoxic than tobramycin $(9,10)$. In the late seventies and early eighties, during the era of multi-daily dosed (MDD) aminoglycoside regimens, human studies also suggested that gentamicin is more nephrotoxic than tobramycin (11). Nevertheless, since none of these studies complied with the methodological standards of a bias-free comparative drug trial, Smith et al. conducted a double-blind randomized clinical trial with larger sample size, showing that MDD gentamicin was indeed more nephrotoxic than MDD tobramycin (12). Related to this matter it is worth mentioning, that no real preference exist for either gentamicin or tobramycin as first line aminoglycoside with regard to worldwide resistance patterns (13-17). Over the past two decades traditional MDD schedules of aminoglycosides have largely been replaced by once daily dosed (ODD) aminoglycoside regimens with relatively more favorable toxicity profile. Aminoglycosides accumulate in renal proximal tubular cells and inner ear tissue $(1,6-8)$. Once daily dosing extends the period of minimal or drug free serum concentration allowing the renal and ear compartment more time to void the accumulated drug. Clinical studies have shown that once daily dosing of aminoglycosides substantially lowers the risk of nephro- and ototoxicity without compromising efficacy (18-20). Strong evidence exists that gentamicin has a higher nephrotoxic potential compared to tobramycin based on animal studies and clinical studies using MDD schedules. However, to date this has never been demonstrated for nowadays more common ODD regimens. Therefore, we investigated the nephrotoxicity and pharmacokinetics of ODD gentamicin versus ODD tobramycin in hospitalized patients with suspected or proven infection with gram-negative micro-organisms. 


\section{Patients and Methods}

In this retrospective cohort study adult patients with nosocomial infections who were administered systemic gentamicin or tobramycin at the Medical Center Haaglanden Westeinde (The Hague, The Netherlands), a 500-bed teaching hospital, between January 2008 and December 2012 were selected from the electronic medication records. Following synchronization of regional antibiotic guidelines in February 2010, the local antibiotic guidelines switched from gentamicin to tobramycin for all indications requiring treatment with an aminoglycoside, without any changes in treatment and monitoring protocol. The selected patients were eligible for inclusion if they were treated with a once daily dose of $4-6 \mathrm{mg} / \mathrm{kg} / \mathrm{day}$ of gentamicin or tobramycin for a minimum of 3 days. Gentamicin and tobramycin were administered as 30 min infusions. According to the monitoring protocol, a peak serum concentration (Cmax) and second concentration were drawn 0.5-1 h and 6-14 h after end of the first or second infusion, respectively. Aminoglycoside dosing was guided by drug concentration monitoring targeting a Cmax of 15-20 $\mathrm{mg} / \mathrm{L}$ and estimated trough concentrations $<0.5 \mathrm{mg} / \mathrm{L}$. In case of trough concentrations $>0.5 \mathrm{mg} / \mathrm{L}$, extended interval dosing was applied. In all other cases subsequent doses were administered every 24 hours. Drug concentrations were monitored at least twice weekly according to protocol. Timings of blood sampling for therapeutic drug monitoring were accurately recorded. Two serum creatinine concentrations were required for inclusion: one drawn within 48 hours before therapy and a minimum of one concentration drawn within 48 hours after initiation and 48 hours after cessation of therapy. Exclusion criteria were pre-existing renal impairment defined as a serum creatinine concentration $\geq 250$ $\mu \mathrm{mol} / \mathrm{L}$ or an estimated glomerular filtration rate (eGFR; calculated with modified diet in renal disease formula (21)) of $\leq 20 \mathrm{ml} / \mathrm{min}$ at baseline, admittance to the intensive care unit, and a diagnosis of endocarditis. A waiver was provided by the local medical ethics committee.

Nephrotoxicity was identified by the acronym RIFLE (Risk, Injury, Failure, Loss of kidney function, and End-stage kidney disease) with the exclusion of urine production values, since these could not consistently be retrieved from patient records (22). The rise in serum creatinine was determined by subtracting the initial concentration from the highest measured concentration drawn between 48 hours after initiation and 
48 hours after cessation of therapy.(12) The incidence of nephrotoxicity was compared between gentamicin- and tobramycin-treated patients. Analogous to the rise in serum creatinine, delta blood urine nitrogen (BUN) was calculated. Pharmacokinetic (PK) parameters of tobramycin and gentamicin were calculated for all individuals using one-compartment open models and PK software (MWIPHARM 3.60, Mediware, The Netherlands) with an iterative two-stage Bayesian fitting procedure.

\section{Statistical analysis}

A Kolmogorov-Smirnov test was used to identify normal distribution of data. Means and standard deviations of continuous variables are presented in normal distributions. Otherwise, medians and ranges were reported. A Student's T-test was used to determine statistical differences for normally distributed data. If data were not normally distributed, a Mann-Whitney-U test was performed. A Chi-square test was used to compare dichotomous variables between groups. Uni- and multivariate logistic regression analyses were performed to identify determinants that were available prior to the start of therapy, i.e. aminoglycoside, gender, age, weight, ward and pretreatment creatinine, BUN and eGFR, prior to the second administration, i.e. Cmax, estimated 24h-concentration, clearance, volume of distribution and the area-under-the-curve of the first administration (AUCfirst) and available at the end of therapy, i.e. cumulative dose and duration of therapy. A stepwise backward elimination procedure was carried out, retaining covariates only if their removal significantly changed the model. To account for multiple testing only those covariates were tested that significantly affected the risk of nephrotoxicity in univariate analyses and a stringent $P$ value $(P<0.005)$ during multivariate analysis was selected. Finally, the predictive value of AUCfirst cut-off values of $120,150,180$ and $210 \mathrm{mg}^{*} \mathrm{~h} / \mathrm{L}$ on nephrotoxicity defined as RIFLE stage $R$ were tested with multivariate regression. All tests were performed in SPSS Statistics version 20 (SPSS Inc., Chicago, IL, USA) and a P-value of $<0.05$ was considered to be statistically significant and in each instance. 


\section{Results}

Of the 2889 courses that were selected, 382 unique patients met the inclusion criteria and nephrotoxicity was compared in 202 and 180 patients who received gentamicin or tobramycin, respectively. Similar numbers and percentages of patients were excluded per inclusion criterion in each treatment group (Figure 1).

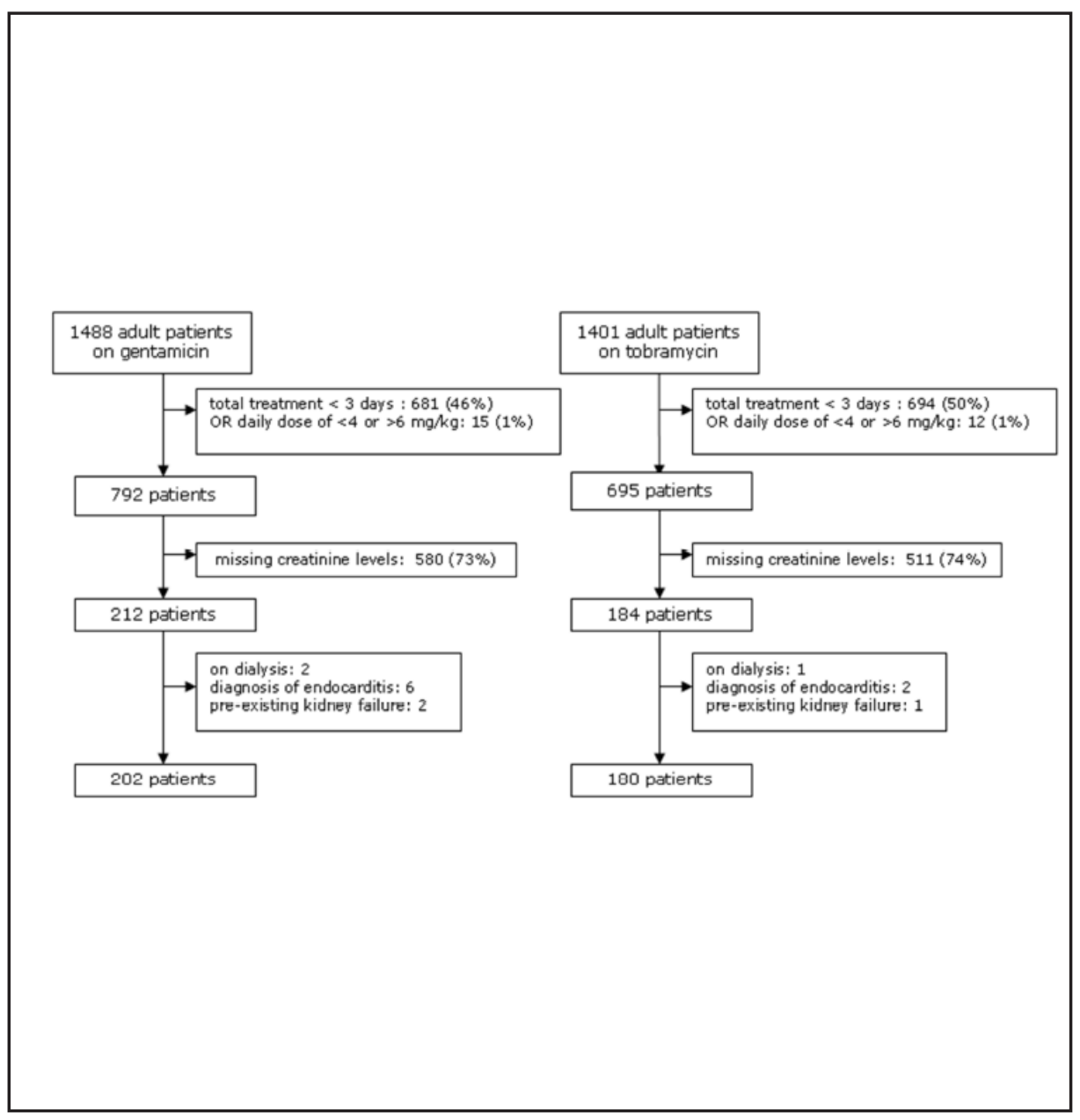


Baseline characteristics of the included patients did not statistically differ between groups (Table 1).

Table 1. Patient characteristics

\begin{tabular}{|c|c|c|c|}
\hline & gentamicin & tobramycin & $\mathrm{p}$-value \\
\hline No. of patients & 202 & 180 & \\
\hline Age (23) & $65(16.2)$ & $63(16.6)$ & $.43 \S$ \\
\hline$\%$ of males & 67 & 72 & $.23^{*}$ \\
\hline Weight (24) & $70(16.8)$ & $69(17.5)$ & $.49 \S$ \\
\hline \multicolumn{4}{|l|}{ Baseline serum } \\
\hline creatinine $(\mathrm{nmol} / \mathrm{mL})$ & $93(37)$ & $89(43)$ & $.33 \S$ \\
\hline eGFR (mL/min) & $75(40)$ & $82(43)$ & $.12 \S$ \\
\hline Ward & & & $.13^{*}$ \\
\hline Surgical (\%) & 42 & 34 & \\
\hline Internal (\%) & 47 & 49 & \\
\hline \multicolumn{4}{|l|}{ Pulmonary } \\
\hline diseases (\%) & 6 & 12 & \\
\hline Other (\%) & 5 & 4 & \\
\hline \multicolumn{4}{|c|}{ Nephrotoxic co-medication } \\
\hline Cefalosporines (\%) & 72.7 & 68.1 & $.34^{*}$ \\
\hline NSAIDs (\%) & 30.3 & 31.9 & $.32^{*}$ \\
\hline Diuretics (\%) & 23.4 & 28.7 & $.24^{*}$ \\
\hline Ciprofloxacin (\%) & 2.2 & 2.1 & $.80^{*}$ \\
\hline Vancomycin (\%) & 1.7 & 3.3 & $.78^{*}$ \\
\hline Erythromycin (\%) & 1.3 & 0 & $.87^{*}$ \\
\hline Azytromicin (\%) & 0.9 & 0 & $.95^{*}$ \\
\hline Cyclosporine (\%) & 0.4 & 0.6 & $.91^{*}$ \\
\hline Indometacin (\%) & 0.4 & 0 & $.96^{*}$ \\
\hline
\end{tabular}

Differences in categorical variables are expressed as numbers and percentages, and continuous variables as means if normally distributed and if not normally distributed as median. Abbreviations are: eGFR, glomerular filtration rate estimated with MDRD formula.

§Student's t-test, ^Mann-Whitney test, ${ }^{\star}$ Chi-squared test 
The incidence of progression to RIFLE stage $\mathrm{R}$ was greater in the gentamicin group with nephrotoxicity developing in 43 of 202 patients $(21.2 \%)$ as compared with 19 of $180(10.6 \%)$ given tobramycin (odds ratio [OR] 2.4, 95\% Cl 1.2-4.7, $\mathrm{P}=0.012$ ). RIFLE stages I and $\mathrm{F}$ were also more frequently observed in patients on gentamicin compared to tobramycin, but the risk was not statistically significant (Table 2).

Table 2. Nephrotoxicity according to RIFLE criteria in patients treated with gentamicin or tobramycin

\begin{tabular}{llllll|}
\hline RIFLE stage & $\begin{array}{l}\text { gentamicin } \\
(\mathrm{n}=202)\end{array}$ & $\begin{array}{l}\text { tobramycin } \\
(\mathrm{n}=186)\end{array}$ & OR & $95 \%-\mathrm{Cl}$ & $\mathrm{p}$-value \\
\hline $\mathrm{R}$ & 43 & 19 & 2.4 & $1.2-4.7$ & .01 \\
$\mathrm{I}$ & 11 & 7 & 1.5 & $0.5-3.8$ & .48 \\
$\mathrm{~F}$ & 5 & 2 & 2.3 & $0.4-12.0$ & .45 \\
\hline
\end{tabular}

Odds ratios for nephrotoxicity defined by the RIFLE classifications using multivariate analysis are presented. Abbreviations are: OR, odds ratio; 95\%-Cl, $95 \%$ confidence interval

Univariate analyses identified gentamicin use $(P=0.006)$, aminoglycoside clearance $(P=0.012)$ and AUCfirst $(P<0.001)$ as independent predictors. During multivariate analysis only gentamicin use $(P=0.08)$ and AUCfirst $(P=0.02)$ were retained by the model as independent predictors of progression to RIFLE stage R. Finally, an AUCfirst over 150 $\mathrm{mg}^{*} \mathrm{~h} / \mathrm{L}$ was associated with an increased risk of developing nephrotoxicity (OR 2.2, 95\% Cl 1.1-5.0, $\mathrm{P}=0.048$ ). These results did not alter after correction for difference in baseline eGFR.

Mean increases in serum creatinine were $13.3 \mathrm{nmol} / \mathrm{mL}$ for the gentamicin group and $0.1 \mathrm{nmol} / \mathrm{mL}$ for patients given tobramycin $(P=0.02)$ (Figure 2). 
Figure 2. Mean increases in serum creatinine in tobramycin and gentamicin treated patients

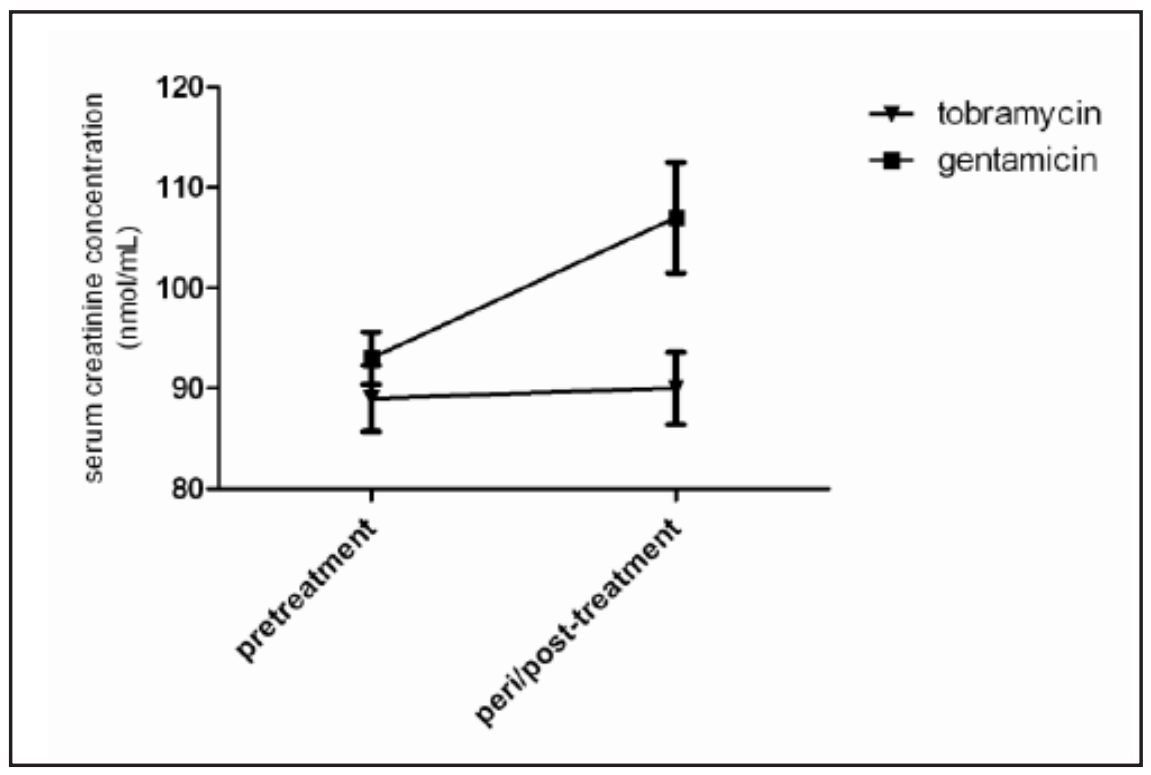

The relative decrease in eGFR between groups showed a trend of a higher loss of eGFR for the patients given gentamicin compared to tobramycin (4.8 vs. $1 \%, p=0.07)$. No significant differences in delta BUN values were noted $(P=0.66)$.

With respect to the pharmacokinetic parameters, the mean AUCfirst of tobramycin was significantly lower compared to gentamicin (98.3 vs. $85.6 \mathrm{mg}^{*} \mathrm{~h} / \mathrm{L}$ respectively, $\mathrm{p}=0.02$ ). The mean peak and estimated trough serum concentrations were within the predefined target range, with higher concentrations in patients treated with gentamicin. Estimation of pharmacokinetic parameters showed a higher mean volume of distribution and clearance of tobramycin compared to mean parameters found in patients on gentamicin (Table 3). 
Table 3. Dosing and Pharmacokinetic parameters

\begin{tabular}{|llll|}
\hline & & & \\
\hline & gentamicin & tobramycin & p-value \\
\hline No. of patients & 175 & 152 & \\
Initial dose(mg) & $330(150-680)$ & $350(140-685)$ & $0.21^{\wedge}$ \\
Cumulative dose (mg) & $1600(1040-7620)$ & $1775(900-6600)$ & $0.20^{\wedge}$ \\
Duration of therapy (days) & $5(3-24)$ & $5(3-22)$ & $0.32^{\wedge}$ \\
AUC of first administration (mg*h/L) & $98.3(45)$ & $85.6(43)$ & $0.02^{\S}$ \\
Volume of distribution (L) & $20.7(7.2)$ & $22.8(6.5)$ & $0.01^{\S}$ \\
Clearance (L/h) & $4.3(2.5)$ & $4.9(2.2)$ & $0.02^{\S}$ \\
$\mathrm{C}_{\text {max }}$ (mg/L) & $17.8(6.5)$ & $16.6(6.2)$ & $0.01^{\S}$ \\
Estimated 24h-concentration & $0.18(5.0)$ & $0.09(3.7)$ & $0.01^{\wedge}$ \\
(mg/L) & & & \\
\hline
\end{tabular}

Differences in categorical variables are expressed as numbers and percentages, and continuous variables as means or medians and standard deviation or range between parentheses. Abbreviations are: AUC, area-under-the-curve and Cmax, peak serum concentration.

$\S$ Student's t-test

${ }^{\wedge}$ Mann-Whitney test

\section{Discussion}

This large retrospective cohort study shows that ODD gentamicin is more nephrotoxic than ODD tobramycin in clinically infected patients who were treated with aminoglycoside therapy for 3 days or longer. The higher nephrotoxic potential of gentamicin was associated with lower clearance and higher AUC's compared to tobramycin offering a biological explanation for this important finding. Furthermore, a high AUC of the first administration was associated with an increased risk of nephrotoxicity. To our knowledge this is the first study directly comparing the nephrotoxic effect among the two most frequently prescribed ODD intravenous aminoglycosides.

Despite the weakness of a retrospective design with consecutive cohorts the risks of both selection- and time bias appear low, since per criterion, similar numbers of patients were excluded, and there were no 
significant differences between patient characteristics of both included and eligible patients (data not shown for the latter). The validity of our outcomes is strengthened by the large cohort investigated, and the hospital-wide switch to tobramycin in all aminoglycoside-indications, explaining similar baseline characteristics in both populations. Nevertheless, future prospective studies remain warranted to confirm these results and are preferably of randomized controlled design.

Whereas the AUC of the first administrion and aminoglycoside clearances were significant univariate predictors of nephrotoxicity, baseline serum creatinine and eGFR were not predictive of developing nephrotoxicity. An explanation for these important secondary findings may be the route of excretion of aminoglycosides. As opposed to creatinine, which is also partially excreted by proximal tubular secretion, aminoglycosides are almost exclusively excreted via glomerular filtration $(25,26)$. Secondly, the 'serum' AUC is expected to be a stronger predictor of nephrotoxicity than clearance, since it correlates best with aminoglycoside exposure in the distal tubuli (27), In fact, the daily AUC has previously been reported. as significant predictor of nephrotoxicity by Rybak et al. (28). And thirdly, high serum creatinine concentrations will trigger the treating physician to initiate close monitoring and to consider extended interval dosing anticipating the increased risk of nephrotoxicity (29). Consequently, the 'a priori' higher risk of nephrotoxicity in patients with poor creatinine clearance will be reduced by the physician's preventive and corrective actions. Additionally, patients with pre-existing renal insufficiency which was a contraindication according to the local treatment protocol received other antibiotic agents. This renal function-based 'triage' methodology is vital to prevent kidney damage by aminoglycosides, but will concomitantly lower the predictive value of creatinine serum concentrations. In our opinion the optimal triage to identify patients who are at high at risk of nephrotoxicity if treated with an aminoglycoside for several days, should consist of baseline eGFR assessment followed by early AUC monitoring.

The randomized trial performed by Smith et al. found no significant and clinically relevant difference in ototoxicity of MDD gentamicin and tobramycin.

Unfortunately, pure-tone-audiometry for measuring hearing loss was not standard of care at our hospital and the risk of ototoxicity could not be investigated. Nevertheless, the low incidences of hearing loss and vertigo in clinically infected patients exposed to aminoglycosides less 
then 7 days demand studies with larger samples sizes and/or novel more sensitive techniques of detecting ototoxicity such as otoacoustic emissions to detect differences between individual aminoglycosides (30).

As mentioned, once daily dosing strategies of $5-7 \mathrm{mg} / \mathrm{kg}$ offer a favourable pharmacokinetic/pharmacodynamic profile and are associated with lower incidence of kidney dysfunction and failure compared to MDD aminoglycoside regimens, since efficacy is assured by higher Cmax values for minimal inhibitory concentrations (MIC's) $<1-2 \mathrm{mg} / \mathrm{L}$ and renal toxicity is minimized by the longer dosing intervals (31). In the current study lower Cmax values for tobramycin were found. Nevertheless, the predefined Cmax targets were attained for both aminoglycosides assuring efficacy. Furthermore, we found that estimated 24 hour concentrations were not predictive of nephrotoxicity. Importantly, the majority of the estimated trough concentrations were far below the limit of detection of currently available aminoglycoside serum concentration assays and these assays show high coefficients of variation in the lower concentration range $(<0.5-1.0 \mathrm{mg} / \mathrm{L})(32)$. Trough concentration monitoring thereby provides only limited information on drug exposure and pharmacokinetics in individual patients (33).

In conclusion, multidrug resistant micro-organisms and a dried up antibiotic pipeline have led to the resurgence of aminoglycosides. As their clinical usage is expected to expand in the years to come, it is of crucial importance to rediscover this class of highly effective antibiotics, and learn how to dose them in both an efficacious and safe manner. Our data suggest that in the once daily dosing era, a further reduction of the incidence of nephrotoxicity may be obtained by preferring tobramycin over gentamicin as first-line aminoglycoside in clinically infected patients. Moreover, the predictive value of 'first administration-AUC' suggests that early therapeutic drug monitoring can aid in reducing aminoglycoside related nephrotoxicity in patients that require treatment for three days or longer. 


\section{Funding}

This work was not supported by outside funding. All work was funded by our department research budget.

\section{Transparency declarations}

None of the authors has conflicts of interest to declare. 


\section{References}

1. Ho J, Tambyah PA, Paterson DL. Multiresistant Gram-negative infections: a global perspective. Curr Opin Infect Dis;23(6):546-53.

2. Gould IM, Bal AM. New antibiotic agents in the pipeline and how they can help overcome microbial resistance. Virulence;4(2):185-91.

3. Williams PD, Bennett DB, Gleason CR, Hottendorf GH. Correlation between renal membrane binding and nephrotoxicity of aminoglycosides. Antimicrob Agents Chemother 1987;31(4):570-4.

4. Schmitz C, Hilpert J, Jacobsen C, Boensch C, Christensen El, Luft FC, et al. Megalin deficiency offers protection from renal aminoglycoside accumulation. J Biol Chem 2002;277(1):618-22.

5. Mingeot-Leclercq MP, Tulkens PM. Aminoglycosides: nephrotoxicity. Antimicrob Agents Chemother 1999;43(5):1003-12.

$6 . \quad$ Lopez-Novoa JM, Quiros Y, Vicente L, Morales Al, Lopez-Hernandez FJ. New insights into the mechanism of aminoglycoside nephrotoxicity: an integrative point of view. Kidney Int 2011;79(1):33-45.

7. Perazella MA. Renal vulnerability to drug toxicity. Clin J Am Soc Nephrol 2009;4(7):1275-83.

8. Tran Ba Huy P, Bernard P, Schacht J. Kinetics of gentamicin uptake and release in the rat. Comparison of inner ear tissues and fluids with other organs. J Clin Invest 1986;77(5):1492-500.

9. Houghton DC, Plamp CE, 3rd, DeFehr JM, Bennett WM, Porter G, Gilbert D. Gentamicin and tobramycin nephrotoxicity. A morphologic and functional comparison in the rat. Am J Pathol 1978;93(1):137-52.

10. Gilbert DN, Plamp C, Starr P, Bennet WM, Houghton DC, Porter G. Comparative nephrotoxicity of gentamicin and tobramycin in rats. Antimicrob Agents Chemother 1978;13(1):34-40.

11. Kahlmeter G, Dahlager JI. Aminoglycoside toxicity - a review of clinical studies published between 1975 and 1982. J Antimicrob Chemother 1984;13 Suppl A:9-22.

12. Smith CR, Lipsky JJ, Laskin OL, Hellmann DB, Mellits ED, Longstreth J, et al. Double-blind comparison of the nephrotoxicity and auditory toxicity of gentamicin and tobramycin. N Engl J Med 1980;302(20):1106-9.

13. Poonsuk K, Tribuddharat C, Chuanchuen R. Aminoglycoside resistance mechanisms in Pseudomonas aeruginosa isolates from non-cystic fibrosis patients in Thailand. Can J Microbiol 2013;59(1):51-6.

14. Vaziri F, Peerayeh SN, Nejad QB, Farhadian A. The prevalence of aminoglycoside-modifying enzyme genes (aac (6')-I, aac (6')-II, ant (2")-I, aph (3')-VI) in Pseudomonas aeruginosa. Clinics (Sao Paulo) 2011;66(9):1519-22.

15. Dubois V, Arpin C, Dupart V, Scavelli A, Coulange L, Andre C, et al. Beta-lactam and aminoglycoside resistance rates and mechanisms among Pseudomonas aeruginosa in French general practice (community and private healthcare centres). J Antimicrob Chemother 2008;62(2):316-23.

16. Clark RB, Sanders CC, Pakiz CB, Hostetter MK. Aminoglycoside resistance among Pseudomonas aeruginosa isolates with an unusual disk diffusion antibiogram. Antimicrob Agents Chemother 1988;32(5):689-92.

17. Mayer $\mathrm{KH}$. Review of epidemic aminoglycoside resistance worldwide. Am J Med 1986;80(6B):56-64.

18. Barza M, loannidis JP, Cappelleri JC, Lau J. Single or multiple daily doses of 
aminoglycosides: a meta-analysis. Bmj 1996;312(7027):338-45.

19. Munckhof WJ, Grayson ML, Turnidge JD. A meta-analysis of studies on the safety and efficacy of aminoglycosides given either once daily or as divided doses. $J$ Antimicrob Chemother 1996;37(4):645-63.

20. Bailey TC, Little JR, Littenberg B, Reichley RM, Dunagan WC. A meta-analysis of extended-interval dosing versus multiple daily dosing of aminoglycosides. Clin Infect Dis 1997;24(5):786-95.

21. Levey AS, Bosch JP, Lewis JB, Greene T, Rogers N, Roth D. A more accurate method to estimate glomerular filtration rate from serum creatinine: a new prediction equation. Modification of Diet in Renal Disease Study Group. Ann Intern Med 1999;130(6):461-70.

22. Bellomo R, Ronco C, Kellum JA, Mehta RL, Palevsky P. Acute renal failure definition, outcome measures, animal models, fluid therapy and information technology needs: the Second International Consensus Conference of the Acute Dialysis Quality Initiative (ADQI) Group. Crit Care 2004;8(4):R204-12.

23. Cano EL, Haque NZ, Welch VL, Cely CM, Peyrani P, Scerpella EG, et al. Incidence of nephrotoxicity and association with vancomycin use in intensive care unit patients with pneumonia: retrospective analysis of the IMPACT-HAP Database. Clinical therapeutics;34(1):149.

24. Bellomo R, Ronco C, Kellum JA, Mehta RL, Palevsky P, Acute Dialysis Quality Initiative w. Acute renal failure - definition, outcome measures, animal models, fluid therapy and information technology needs: the Second International Consensus Conference of the Acute Dialysis Quality Initiative (ADQI) Group. Critical Care (London, England) 2004;8(4):R204.

25. Ferguson MA, Waikar SS. Established and emerging markers of kidney function. Clin Chem 2012;58(4):680-9.

26. Zarowitz BJ, Robert S, Peterson EL. Prediction of glomerular filtration rate using aminoglycoside clearance in critically ill medical patients. Ann Pharmacother 1992;26(10):1205-10.

27. Croes S, Koop AH, van Gils SA, Neef C. Efficacy, nephrotoxicity and ototoxicity of aminoglycosides, mathematically modelled for modelling-supported therapeutic drug monitoring. Eur J Pharm Sci 2012;45(1-2):90-100.

28. Rybak MJ, Abate BJ, Kang SL, Ruffing MJ, Lerner SA, Drusano GL. Prospective evaluation of the effect of an aminoglycoside dosing regimen on rates of observed nephrotoxicity and ototoxicity. Antimicrob Agents Chemother 1999;43(7):1549-55.

29. Maglio D, Nightingale CH, Nicolau DP. Extended interval aminoglycoside dosing: from concept to clinic. Int J Antimicrob Agents 2002;19(4):341-8.

30. Prayle A, Watson A, Fortnum $\mathrm{H}$, Smyth A. Side effects of aminoglycosides on the kidney, ear and balance in cystic fibrosis. Thorax 2010;65(7):654-8.

31. Drusano GL, Ambrose PG, Bhavnani SM, Bertino JS, Nafziger AN, Louie A. Back to the future: using aminoglycosides again and how to dose them optimally. Clin Infect Dis 2007;45(6):753-60.

32. Touw DJ, Westerman EM, Sprij AJ. Therapeutic drug monitoring of aminoglycosides in neonates. Clin Pharmacokinet 2009;48(2):71-88.

33. Jelliffe RW, Iglesias T, Hurst AK, et al. Individualising gentamicin dosage regimens: a comparative review of selected models, data fitting methods and monitoring strategies. Clin Pharmacokinet 1991;21:461-78. 


\title{
Chapter 2.4
}

Extended-interval dosing of gentamicin in neonates targeting trough concentrations under $0.5 \mathrm{mg} / \mathrm{L}$ :

how low can we go?

\author{
ERIK VAN MAARSEVEEN \\ ARWEN SPRIJ \\ DANIEL TOUW
}

submitted

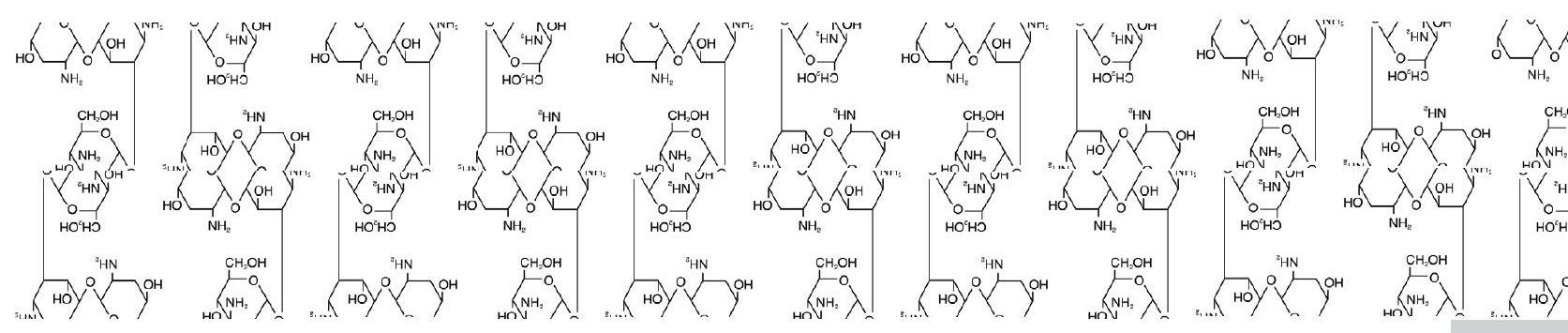




\begin{abstract}
Gentamicin-based regimens are often used in neonatal sepsis but should be used with care because of the narrow therapeutic index and potentially serious toxicity of gentamicin. In the adult populations, it is common to aim for a high maximum concentration (Cmax) and a drug-free period (concentration $<0.5 \mathrm{mg} / \mathrm{L}$ ) of at least 4 hours to prevent toxicity. Albeit similar data in neonates are unavailable, there is no evidence that extended interval administration with target serum concentrations of $<0.5 \mathrm{mg} / \mathrm{L}$ cannot be applied in this population. Therefore, the primary aim of this study was to prospectively asses the target attainment of a simple and practical dosing regimen of $5 \mathrm{mg} /$ $\mathrm{kg} / 36 \mathrm{~h}$ aiming for trough concentrations $<0.5 \mathrm{mg} / \mathrm{L}$. In this prospective observational study neonates admitted to a level II neonatal nursery diagnosed with (suspected) early onset sepsis and commencing intravenous gentamicin therapy were included. According to the monitoring protocol, a peak concentration and second concentration were drawn 0.5-1 $\mathrm{h}$ and 6-18 $\mathrm{h}$ after end of the first or second infusion, respectively. Gentamicin dosing was guided by drug concentration monitoring targeting $\mathrm{Cmax}$ values $>8 \mathrm{mg} / \mathrm{L}$, and estimated trough concentrations $<0.5 \mathrm{mg} / \mathrm{L}$. After exclusion of thirty patients because of insufficient data $(\mathrm{N}=11), \mathrm{GA}<32$ weeks $(\mathrm{N}=7)$ and $\mathrm{PNA}>7$ days $(\mathrm{N}=12)$ a total of 184 unique patients were included for analysis. $90.4 \%$ of patients $(\mathrm{N}=166)$ achieved a Cmax value $>8 \mathrm{mg} / \mathrm{L}$ and a $\mathrm{Cmin}<0.5 \mathrm{mg} / \mathrm{L}$. In conclusion, the dosing regimen showed good performance with respect to target attainment in neonates admitted to a level II unit. Clinical studies investigating the pharmacokinetics and risk/benefit ratio of dosing strategies aiming for drug-free periods ( $\mathrm{Cmin}<0.5 \mathrm{mg} / \mathrm{L})$ in neonatal populations are warranted.
\end{abstract}

\title{
Introduction
}

Early-onset neonatal sepsis occurs in the first seven days of life predominantly due to contaminated amniotic fluid or blood with predominant pathogens such as group B streptococcal (GBS), Escherichia coli, enterococci, Listeria monocytogenes, or other gram negative bacilli 
(1) and is one of the primary causes of mortality among neonates (2). Current guidelines for empirical therapy in neonates with (suspected) sepsis recommend gentamicin-based regimens in preference to cefotaxime-based treatments, because of higher concentrations of susceptibility to gentamicin and the necessity to circumvent exerting selective pressure for resistance (3).Although the combination of the aminoglycoside gentamicin and a penicillin antibiotic has proven to be an effective treatment for staphylococcal species, enterococci and GBS infections (4), it should be used with care because of the narrow therapeutic index and potentially serious toxicity of gentamicin (5). Neonates have aberrant pharmacokinetics (PK) with high inter-patient variability compared to adult patients. As their kidneys are still in development, aminoglycoside clearance and glomerular filtration rate are correlated to gestational age (GA) and postnatal age (PNA) (6). Furthermore, over $70 \%$ of their body weight consists of water, which increases the volume of distribution (L/kg) of aminoglycosides (7). In neonates target peak serum concentrations (Cmax) range from 5 to $12 \mathrm{mg} / \mathrm{L}$ and trough concentrations (Cmin) from 0.5 to $2 \mathrm{mg} / \mathrm{L}$. In contrast, in the adult population, it is common to aim for a Cmax of $20 \mathrm{mg} / \mathrm{L}$ and a drug-free period (concentration $<0.5 \mathrm{mg} / \mathrm{L}$ ) of at least 4 hours (8). Although similar data in neonates are unavailable, there is no evidence that extended interval administration with target trough serum concentrations of $<0.5 \mathrm{mg} / \mathrm{L}$ cannot be applied in this population (5). Another approach advocated in literature is area under the concentration-time curve (AUC) guided dosing. The AUC is believed to be a strong predictor of (nephro)toxicity (9) and the AUC over minimal inhibitory concentration (MIC) ratio is the surrogate pharmacodynamic index that correlates best with resistance suppression and treatment success (10). Albeit there are limited clinical data on the optimal gentamicin exposure in terms of AUC, it has been suggested that aiming for a $90 \%$ probability of a treatment success and under $10 \%$ probability of toxicity, an AUC24h value of $75 \mathrm{mg} . \mathrm{h} / \mathrm{L}$ should be aimed for when pathogen's MIC's are between 0.5 or 1.0 $\mathrm{mg} / \mathrm{L}$ (9). The majority of current dosing regimens of aminoglycosides in neonates are based on data obtained from neonates admitted to level III intensive care unit also known as a neonatal intensive care unit (NICU). As these critically ill sometime very premature newborns show a large inter-patient variability in PK parameters, most dosing regimens consist of complex algorithms with different dosages and intervals depending on GA, PNA and/or body weight (5). It is, however, debatable whether complex algorithms with known high risk of prescription 
errors (11) are required to attain predefined targets in non-critically ill term neonates admitted to a level II special care unit. The inter-patient variability in $\mathrm{PK}$ parameters is expected to be lower in this population in comparison with neonates admitted to a NICU due to a generally better clinical condition of the former. Therefore, the two primary aims of this study were (1) to prospectively asses the target attainment of a simple and practical dosing regimen of $5 \mathrm{mg} / \mathrm{kg} / 36 \mathrm{~h}$ aiming for low trough concentrations $(<0.5 \mathrm{mg} / \mathrm{L})$ and $(2)$ to evaluate inter-patient $\mathrm{PK}$ variability in neonates admitted to a level II neonatal special care unit.

\section{Methods}

\section{Study design and patients}

In this prospective observational study neonates admitted to the level II neonatal nursery at the Juliana Children's Hospital (The Hague, The Netherlands) diagnosed with (suspected) early onset sepsis and commencing intravenous gentamicin therapy between January 2010 and January 2013 were eligible for inclusion. Main exclusion criteria were GA $<32$ weeks and PNA $>7$ days. A waiver for consent was provided by the regional medical ethics committee.

\section{Gentamicin dosing and monitoring}

A priori gentamicin was dosed $5 \mathrm{mg} / \mathrm{kg}$ every 36 hours administered as a $30 \mathrm{~min}$ infusion in combination with amoxicillin $100 \mathrm{mg} / \mathrm{kg}$ twice daily. According to the monitoring protocol, a peak concentration and second concentration were drawn $0.5-1 \mathrm{~h}$ and $6-18 \mathrm{~h}$ after end of the first infusion, respectively. Gentamicin dosing was guided by drug concentration monitoring targeting $\mathrm{Cmax}$ values $>8 \mathrm{mg} / \mathrm{L}$, and estimated trough concentrations $<0.5 \mathrm{mg} / \mathrm{L}$. Dose adjustments were communicated by a clinical pharmacist before administration of the second dose.In case of estimated trough concentrations $>0.5 \mathrm{mg} / \mathrm{L}$, the dosing interval was extended. In all other cases subsequent doses were administered every 36 hours. Drug concentrations were monitored at least once every other dose according to protocol. Antibiotic treatment was stopped if blood cultures showed no bacterial growth within 3 days. Concentrations of gentamicin were measured using an automated chemiluminescent immunoassay (Architect c8000, Abbott Laboratories, Amstelveen, The Netherlands). The coefficient of variation for the gentamicin assay was less than $5 \%$ in the range of 1 to $10 \mathrm{mg} / \mathrm{L}$. The limit of quantificati- 
on (LOQ) was $0.3 \mathrm{mg} / \mathrm{L}$.

\section{Data collection and exposure analysis}

The following demographic and clinical parameters were collected and registered in patients' clinical records: date of birth, gender, body weight, GA, PNA, Apgar score, date, time and dose of gentamicin administration and blood culture results. Furthermore, individual Cmax, Cmin, AUC, clearance $(\mathrm{Cl})$ and volume of distribution $(\mathrm{Vd})$ values were estimated using a one-compartment open model and PK software (MWIPHARM version 3.60, Mediware, The Netherlands) with an iterative two-stage Bayesian fitting procedure. The performance of the dosing table was assessed by calculation of the percentage of patients attaining the predefined target exposure.

\section{Statistical analysis}

Relationships between BW, GA, PNA and PK parameters were analyzed using the Pearson's correlation test. Furthermore, uni- and multivariate logistic regression analyses were performed to identify covariates predictive of Cmax target attainment failure. A stepwise backward elimination procedure was carried out, retaining covariates only if their removal significantly changed the model $(P<0.005)$. To account for multiple testing only those covariates were tested that significantly affected the risk of target attainment failure in univariate analyses and a stringent $P$ value of 0.005 during multivariate analysis was selected. All tests were performed in SPSS Statistics version 20 (SPSS Inc., Chicago, IL, USA) and a P-value of $<0.05$ was considered to be statistically significant and in each instance.

\section{Results}

During the 3-year enrolment-period 214 unique patients were diagnosed with (suspected) early-onset sepsis and treated with gentamicin treatment episodes. After exclusion of thirty patients because of insufficient data $(\mathrm{N}=11), \mathrm{GA}<32$ weeks $(\mathrm{N}=7)$ and $\mathrm{PNA}>7$ days $(\mathrm{N}=12)$ a total of 184 unique patients ( $53 \%$ of male gender) were included for analysis. Their GA and PNA ranged from 32 to 42 weeks and 0 to 6 days with a median of 39 weeks and 0 days, respectively. Median body weight was $3.15 \mathrm{~kg}$ ranging from $1.2 \mathrm{~kg}$ to $5.1 \mathrm{~kg}$. Median Apgar score 10 minutes postpartum was 9 ranging from 3-10. 
As shown in Table 1,90.4\% of patients $(\mathrm{N}=166)$ achieved both a $\mathrm{Cmax}$ value $>8 \mathrm{mg} / \mathrm{L}$ and a $\mathrm{Cmin}<0.5 \mathrm{mg} / \mathrm{L}$. Because of the high attainment rate with respect to the $\mathrm{Cmin}$ target, it was decided to estimate $\mathrm{C} 24 \mathrm{~h}$ values and assess the target attainment as previously described for the eCmin values at 36 hours. Furthermore, inter-patient variability in clearance, half life and volume of distribution were $30,19.3$ and $16.7 \%$, respectively. Next, significant correlations were found between GA and Cmax ( $r$ 0.44, $\mathrm{P}<0.001)$ and between $\mathrm{GA}$ and $\mathrm{Cmin}(\mathrm{r} 0.42, \mathrm{P}<0.001)$, between BW and $C \max (r 0.58, P<0.001)$ and between BW and $C \min$ ( $r$ 0.50, $\mathrm{P}<0.001$ ). Correlations between AUC $\mathrm{T}$ and $\mathrm{GA}$ ( $\mathrm{r} 0.028, \mathrm{P}=0.7$ ) and between AUCT and BW ( $\mathrm{r} 0.064, \mathrm{P}=0.4$ ) were not significant (Figure 1). Finally, univariate binary logistic regression showed that both low body weight $(P<0.001)$ and low $G A(P<0.001)$ were significant predictors of $\mathrm{Cmax}$ target attainment failure. During multivariate analysis only GA was retained as an independent predictor of underexposure, meaning that neonates in the lower GA range would require relatively higher dose per kg body weight to achieve a Cmax value over $8 \mathrm{mg} / \mathrm{L}$. The patients $(\mathrm{N}=16)$ who did not reach $\mathrm{Cmax}$ values $>8 \mathrm{mg} / \mathrm{L}$ had a Cmax between 4.9 and $7.9 \mathrm{mg} / \mathrm{L}$. Body weights in 11 of these patients were under $2.7 \mathrm{~kg}$ (the first quartile) and 12 had a GA $<35$ weeks (the first quartile).

Blood culture results were positive in 5 of 186 patients (2.7\%); Coagulase-negative staphylococcus (CNS) $(\mathrm{N}=4)$ or Corynebacterium species $(N=1)$, three of which were considered to be positive due to contamination. No cases of ot- or renal toxicity were reported. 
Table 1. Dosing, pharmacokinetics and target attainment rates

\begin{tabular}{|ll|}
\hline Dosing\& PK parameters & Mean (S.D.) \\
\hline Gentamicin dose $(\mathrm{mg})$ & $15.6(4.0)$ \\
$\mathrm{eC}_{\max }(\mathrm{mg} / \mathrm{L})$ & $10.8(1.75)$ \\
$\mathrm{eC}_{\min }(\mathrm{mg} / \mathrm{L})$ & $0.18(0.12)$ \\
$\mathrm{eC}_{24 \mathrm{~h}}(\mathrm{mg} / \mathrm{L})$ & $0.64(0.29)$ \\
$\mathrm{AUC}_{\mathrm{T}}$ & $99(17.1)$ \\
Volume of distribution $(\mathrm{L} / \mathrm{kg})$ & $0.45(0.075)$ \\
Half life $(\mathrm{h})$ & $5.7(1.1)$ \\
$\mathrm{Clearance}(\mathrm{L} / \mathrm{h} / \mathrm{kg})$ & $0.17(0.051)$ \\
& \\
Target $_{\text {attainment }}$ & Rate (\%) \\
$\mathrm{C}_{\max }>8 \mathrm{mg} / \mathrm{L}$ & $168 / 184(91.3)$ \\
$\mathrm{C}_{\min }<0.5 \mathrm{mg} / \mathrm{L}$ & $179 / 184(97.3)$ \\
$\mathrm{C}_{\max }>8 \mathrm{mg} / \mathrm{L}$ and $\mathrm{C}_{\min }<0.5 \mathrm{mg} / \mathrm{L}$ & $166 / 184(90.4)$ \\
$\mathrm{C}_{\min }<1 \mathrm{mg} / \mathrm{L}$ & $184 / 184(100)$ \\
$\mathrm{C}_{24 \mathrm{~h}}<1 \mathrm{mg} / \mathrm{L}$ & $158 / 184(85.9)$ \\
$\mathrm{C}_{24 \mathrm{~h}}<0.5 \mathrm{mg} / \mathrm{L}$ & $95 / 184(51.6)$ \\
& \\
\hline
\end{tabular}

Values are presented as means with standard deviations (S.D.) and ranges or as rates and percentages. Abbreviations are eCmax, maximum serum concentration; eCmin, minimum serum concentration, eC24h, estimated serum concentration $24 \mathrm{~h}$ after end of infusion; $A \cup C \mathrm{~T}$, area under the concentration-time curve of one dosing interval and $\mathrm{Vd}$, volume of distribution. 
Figure 1. Correlations between body weight, gestational age and exposure parameters

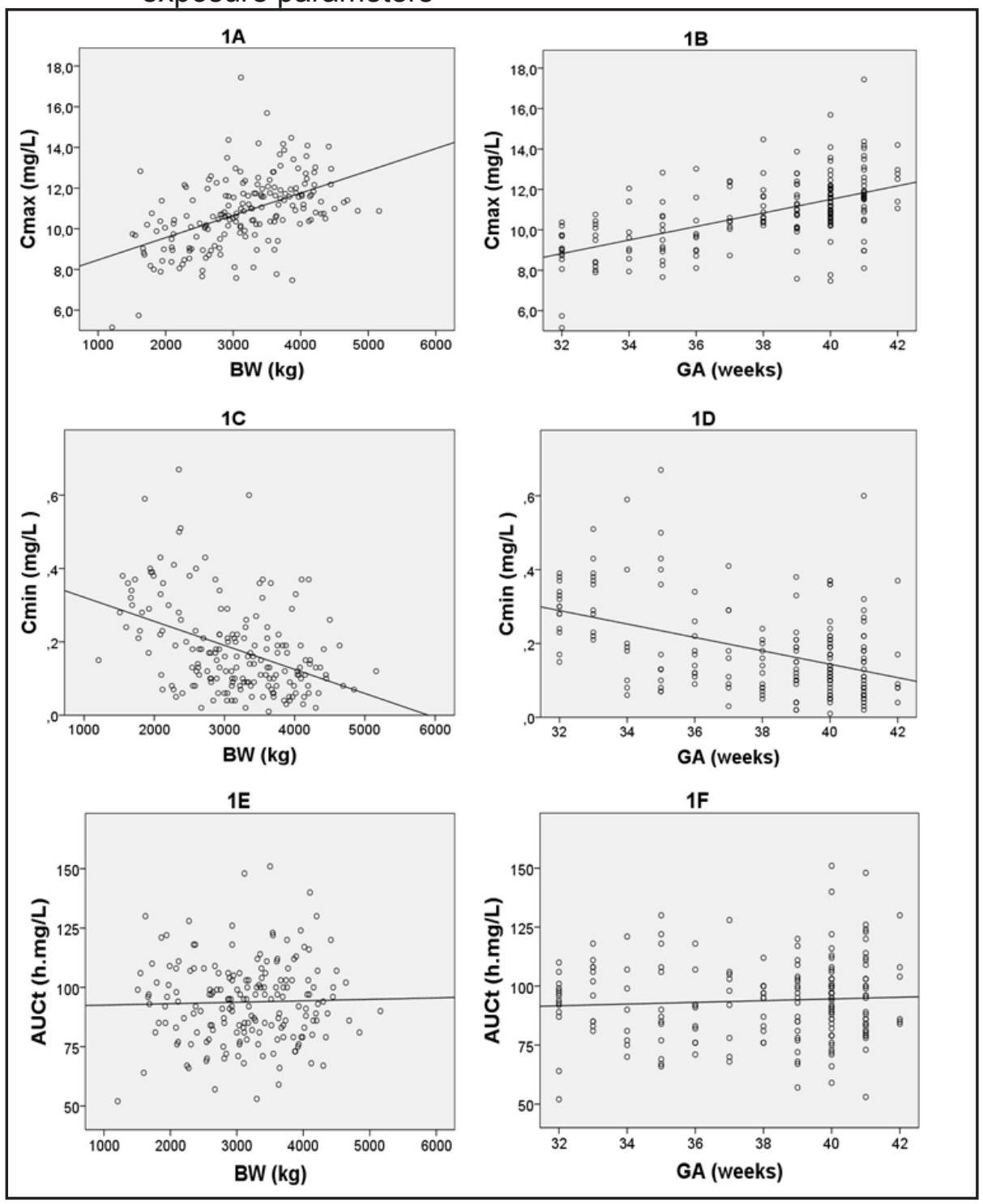

Correlations between BW and $\mathrm{Cmax}$ (Figure 1A, $\mathrm{r} 0.58, \mathrm{P}<0.001$ ) and between $\mathrm{GA}$ and $\mathrm{Cmax}$ (Figure 1B, r 0.44, $\mathrm{P}<0.001$ ), between $\mathrm{BW}$ and $\mathrm{Cmin}$ (Figure 1C, $\mathrm{r} 0.50, \mathrm{P}<0.001$ ) and between GA and $\mathrm{Cmin}$ (Figure 1D, r 0.42, P<0.001), between BW and AUCT (Figure 1E, r 0.064, P=0.4) and between GA and AUCт (Figure 1F, r 0.028, P=0.7) are depicted. Abbreviations are BW, body weight; GA, gestational age; Cmax, maximum serum concentration; Cmin, minimum serum concentration; and $\mathrm{AUCT}$, area under the concentration-time curve of one dosing interval. 


\section{Discussion}

In this prospective clinical study over ninety percent of the included patients reached the combined predefined $\mathrm{Cmax}$ and $\mathrm{Cmin}$ targets using a simple uniform dose of $5 \mathrm{mg} / \mathrm{kg}$ gentamicin every 36 hours aiming for low Cmin values ( $<0.5 \mathrm{mg} / \mathrm{l}$ ) in all neonates admitted to our level II unit with (suspected) early-onset sepsis. The good performance of the gentamicin dosing regimen is associated to the low inter-patient variability in population PK parameters relatively other reports of gentamicin PK in neonates $(5,12,13)$. It should be noted, that most of the latter reports included neonates admitted to a level III unit. They are inherently prone to have a high variability in PK due to severe (underlying) pathology and to a wider range in GA and PNA which in turn are associated with a larger range of birth weight and developmental stage compared to neonates admitted to a level II unit (5). This is confirmed by our findings that low GA and BW were predictive of Cmax target attainment failure.

As neonates with a GA over 32 weeks and diagnosed with (suspected) early onset sepsis (PNA $<7$ days) were included in this study, patients suffering from (suspected) late onset neonatal sepsis were not included as well as preterm neonates with a low GA ( $<32$ weeks). The main exclusion criteria could therefore be considered as the most important limitations of the study outcomes. Yet, only seven neonates were excluded due to a GA under 32 weeks and no more than twelve patients had a PNA over 7 days at start of gentamicin therapy, which is less than $10 \%$ of the total population. These patients should have been referred to a NICU according to international guidelines (14) and the studied gentamicin dosing regimen was not intended to be used in these patients. In clinical practice, we suggest using a dosing algorithm specifically designed in a population of neonates with a GA under 32 weeks. For the twelve neonates with a PNA $>7$ days, a post hoc analysis was performed, which indicated that 10 out of twelve patients (82\%) attained predefined Cmax and Cmin targets (data not shown). Although the sample size was too small to statistically compare the outcomes to neonates with a PNA $<7$ days at the initiation of therapy, these preliminary results suggest the dosing regimen may also show good performance in neonates with a PNA $>7$ days admitted to a level II unit.

One of the major strengths of this study is the combination of a prospective observational design with the minimal exclusion and loss of 
data reflecting real-life clinical practice. Secondly, two plasma samples were drawn at sampling times optimal for accurate estimation of PK parameters. Using this approach, $\mathrm{Cmin}$ and AUC values can be accurately estimated instead of sampling actual trough concentrations that are unreliable due to a high assay error at low gentamicin concentrations (15). This limited AUC sampling approach offers the opportunity to identify individual clearance next to the gentamicin 'load' represented by the AUC. In this study a mean gentamicin 'load' expressed as the AUC36h of $99 \mathrm{mg}^{*} \mathrm{~h} / \mathrm{L}$ was found. After correction for the extended interval a $5 \mathrm{mg} / \mathrm{kg}$ dose every 36 hours is a gentamicin 'load' equivalent to an AUC of $75 \mathrm{mg} . \mathrm{h} / \mathrm{L}$ every 24 hours proposed by Drusano and co-workers that gives high chances of success and safety (9).

Interestingly, the high target attainment rates may call for a revision of the intensive drug concentration monitoring after the first dose in all neonates $>32$ weeks GA and $<7$ days PNA. Sampling after the second dose could significantly reduce patients' blood sampling burden, since gentamicin is frequently administered for a short period of 36 to 72 hours in neonates (16). A further reduction in gentamicin course duration may be anticipated, since the turn-around-times of blood cultures have significantly been reduced in recent years. Especially, in combination with extended dosing interval dosing regimens this could facilitate current practices of stopping gentamicin therapy earlier, perhaps even before the second dose (16-18). Patients will not needlessly be exposed to gentamicin for longer periods, but will also be refrained from both the pain and risk of infection associated to blood sampling in neonates. Two meta-analyses that comprise approximately 600 newborns have shown that irrespective of the dosing schedule in combination with routine aminoglycoside blood concentration monitoring, sepsis was clinically cured in almost every patient $(19,20)$. In this context, one should realize that antibacterial courses are started in newborns even with a low probability of sepsis. Although many studies did not find any aminoglycoside-related toxicity, (transient) hearing loss and renal tubular damage have been reported $(19,21)$. Unfortunately, adequate diagnostic instruments are currently unavailable to sensitively and specifically identify aminoglycoside-toxicity in neonates. Currently, gentamicin trough concentrations or AUC's can be considered the best clinical 'markers' for toxicity monitoring (5).

Because of the pharmacokinetic/pharmacodynamic profile of gentamicin and the rapid dose-dependent killing after the first dose, followed by adaptive resistance and a post-MIC effect, low trough concentrations 
do not necessarily lead to a poorer outcome. A relatively drug-free interval as common in adult populations may reduce aminoglycoside-related toxicity and adaptive resistance in newborns (8). Hence, future clinical studies investigating the pharmacokinetics and risk/benefit ratio of dosing strategies aiming for drug-free periods (Cmin $<0.5 \mathrm{mg} / \mathrm{L})$ in neonatal populations are warranted. 
References

1. Fanos V, Dall'Agnola A. Antibiotics in neonatal infections: a review. Drugs 1999;58(3):405-27.

2. Black RE, Cousens S, Johnson HL, Lawn JE, Rudan I, Bassani DG, et al. Global, regional, and national causes of child mortality in 2008: a systematic analysis. Lancet 2010;375(9730):1969-87.

3. Muller-Pebody B, Johnson AP, Heath PT, Gilbert RE, Henderson KL, Sharland $M$. Empirical treatment of neonatal sepsis: are the current guidelines adequate? Arch Dis Child Fetal Neonatal Ed;96(1):F4-8.

4. Dawson PM. Vancomycin and gentamicin in neonates: hindsight, current controversies, and forethought. J Perinat Neonatal Nurs 2002;16(2):54-72.

5. Touw DJ, Westerman EM, Sprij AJ. Therapeutic drug monitoring of aminoglycosides in neonates. Clin Pharmacokinet 2009;48(2):71-88.

6. Besunder JB, Reed MD, Blumer JL. Principles of drug biodisposition in the neonate. A critical evaluation of the pharmacokinetic-pharmacodynamic interface (Part I). Clin Pharmacokinet 1988;14(4):189-216.

7. Koren $\mathrm{G}$. Therapeutic drug monitoring principles in the neonate. National Academy of CLinical Biochemistry. Clin Chem 1997;43(1):222-7.

8. Nicolau DP, Freeman CD, Belliveau PP, Nightingale CH, Ross JW, Quintiliani R. Experience with a once-daily aminoglycoside program administered to 2,184 adult patients. Antimicrob Agents Chemother 1995;39(3):650-5.

9. Drusano GL, Ambrose PG, Bhavnani SM, Bertino JS, Nafziger AN, Louie A. Back to the future: using aminoglycosides again and how to dose them optimally. Clin Infect Dis 2007;45(6):753-60.

10. Pagkalis S, Mantadakis E, Mavros MN, Ammari C, Falagas ME. Pharmacological considerations for the proper clinical use of aminoglycosides. Drugs 2011;71(17):2277-94.

11. M O'Meara EL. An audit of prescribing errors in neonates and paediatrics. Abstracts from the Poster and Oral presentations from the 18th Neonatal and Paediatric Pharmacists Group (NPPG) Annual Conference held at the Liverpool Marriott Hotel from 9-11 November 20122013.

12. Begg EJ, Vella-Brincat JW, Robertshawe B, McMurtrie MJ, Kirkpatrick CM, Darlow B. Eight years' experience of an extended-interval dosing protocol for gentamicin in neonates. J Antimicrob Chemother 2009;63(5):1043-9.

13. Fjalstad JW, Laukli E, van den Anker JN, Klingenberg C. High-dose gentamicin in newborn infants: is it safe? Eur J Pediatr.

14. Levels of neonatal care. Pediatrics 2012;130(3):587-97.

15. Jelliffe RW, Iglesias T, Hurst AK, et al. Individualising gentamicin dosage regimens: a comparative review of selected models, data fitting methods and monitoring strategies. Clin Pharmacokinet 1991;21:461-78.

16. Chattopadhyay B. Newborns and gentamicin--how much and how often? J Antimicrob Chemother 2002;49(1):13-6.

17. Thureen PJ, Reiter PD, Gresores A, Stolpman NM, Kawato K, Hall DM. Onceversus twice-daily gentamicin dosing in neonates $>/=34$ Weeks' gestation: cost-effectiveness analyses. Pediatrics 1999;103(3):594-8.

18. Stolk LM, Degraeuwe PL, Nieman FH, de Wolf MC, de Boer A. Population pharmacokinetics and relationship between demographic and clinical variables and 
pharmacokinetics of gentamicin in neonates. Ther Drug Monit 2002;24(4):527-31.

19. Nestaas E, Bangstad HJ, Sandvik L, Wathne KO. Aminoglycoside extended interval dosing in neonates is safe and effective: a meta-analysis. Arch Dis Child Fetal Neonatal Ed 2005;90(4):F294-300.

20. Rao SC, Ahmed M, Hagan R. One dose per day compared to multiple doses per day of gentamicin for treatment of suspected or proven sepsis in neonates. Cochrane Database Syst Rev 2006(1):CD005091.

21. Borradori C, Fawer CL, Buclin T, Calame A. Risk factors of sensorineural hearing loss in preterm infants. Biol Neonate 1997;71(1):1-10. 



\section{Chapter 3}

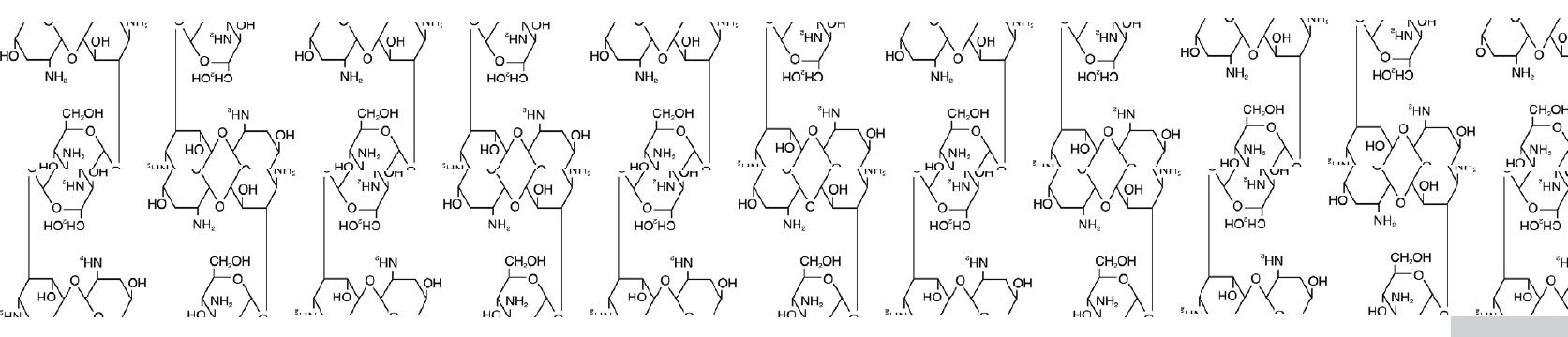




\title{
Chapter 3.1
}

\section{Vancomycin Exposure Variability of Intermittent versus Continuous Infusion: a systematic review}

\author{
SUZAN G.H. GIPMANS \\ ERIK M. VAN MAARSEVEEN \\ ARTHUR R.H. VAN ZANTEN
}

Submitted

sing
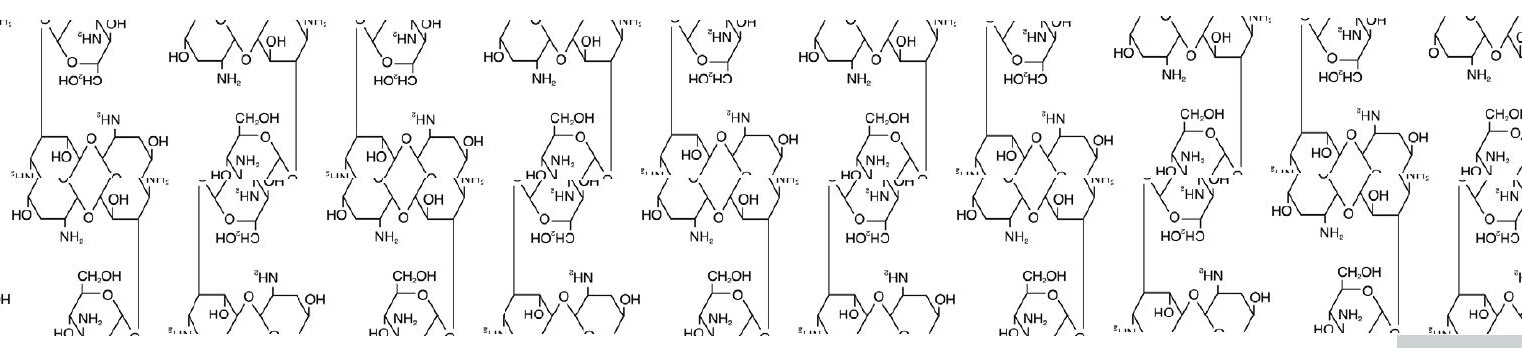

Chapter 3.1 


\section{Abstract}

This systematic review aims to compare exposure variability and target attainment of vancomycin intermittent infusion ( $\mathrm{Inl}$ ) and continuous infusion (Col). A literature search was performed and clinical studies reporting on vancomycin treated populations were selected. After exclusion of reviews, case-reports and articles not published in the English language, 505 articles were screened for data on vancomycin serum concentrations. A total of 34 studies were included for review. A comparison of relative standard deviations (RSD) of vancomycin serum concentrations was performed to quantify Inl and Col exposure variability. The variability in serum concentrations was significantly larger for Inl than Col (RSD 46.5 and $32.1 \%$, respectively; $p=0.001$ ). Exposure variability appeared to be independent of the study population or design. Studies directly comparing target attainment between both modes of administration denoted higher and faster target attainment with Col. In conclusion, Col was associated with lower exposure variability and favourable target attainment rates compared to Inl. It remains, however, to be elucidated whether vancomycin Col results in better clinical outcomes compared to Inl.

\section{Introduction}

The glycopeptide antibiotic vancomycin was developed in the 1950s, when penicillin-resistant Staphylococcus aureus called for new antibiotics. In the early years of its development, vancomycin was known to cause nephrotoxicity and ototoxicity as well as infusion-related toxicities. These toxicities were most likely caused by impurities in the formulation, which gave it a brownish appearance and vancomycin was therefore also dubbed 'Mississippi mud' (1). After the fermentation process was changed, yielding purer vancomycin, the rate of toxicity was markedly reduced (2). Primarily, the clinical use of vancomycin declined when semisynthetic penicillins such as methicillin, oxacillin and nafcillin became available, which were considered to be less toxic. However, in the 1970s vancomycin regained interest as the result of the rise of methicillin resistant Staphylococcus aureus (MRSA) infections $(1,3)$. Currently, vancomycin is the drug of choice in the treatment of severe MRSA infections such as sepsis, endocarditis, osteomyelitis 
and health care-associated or community-acquired pneumonia (4), as well as infections caused by other gram-positive bacteria. Usually, vancomycin is administered intermittently and serum concentrations are monitored (4). However, it has been suggested that continuous infusion of vancomycin may reduce the rates of nephrotoxicity without compromising efficacy and target serum concentrations may be attained faster (5). The studies comparing clinical outcomes between intermittent (InI) and continuous (Col) infusion of vancomycin were recently reviewed in a meta-analysis by Cataldo et al. (6). A significant lower rate of nephrotoxicity was observed with Col compared to Inl in this meta-analysis. However, no differences in mortality were noted and the definition of efficacy was inconsistent in the included studies to pool efficacy outcome measures (6). An alternative strategy to compare Inl and Col of vancomycin is to compare surrogate outcome parameters e.g. variability in drug exposure or target attainment rates. Minimal interpatient variability in exposure and rapid target attainment are important aims of any vancomycin dosing strategy striving for maximum efficacy and minimal toxicity. Therefore, the present review aims to compare the variability of exposure of Inl and Col in vancomycin treated populations using serum concentrations in published data. Furthermore, target attainment with either mean of administration will be assessed.

\section{Methods}

To assess the variability of exposure and target attainment of Inl and Col vancomycin treatment, a literature search in PubMed/MEDLINE (1950 to February 2014), Web of Science (1900 to February 2014) and Embase (1947 to February 2014) was conducted and the reference lists of included articles were screened manually. The index search terms in PubMed were 'vancomycin' AND (serum concentration OR level $^{*}$ OR concentration*) AND (dosing OR intravenous) and the search was limited to articles published in English and studies performed in humans. An adapted search was performed in Embase and Web of Science. Clinical studies, as well as pharmacodynamic and pharmacokinetic studies were eligible for inclusion. Studies with the following criteria were excluded: paediatric data, data on haemodialysis, and studies that did not report serum concentrations or area under the concentration-time curve $(A \cup C)$ (mean $\pm S D$ ) values.

From the included articles, data on target attainment rates and serum 
concentrations or AUCs (mean $\pm S D$ ) were extracted. Next, the variabilities in AUC, trough and 'plateau' serum concentrations was determined by the reported relative standard deviation (RSD). If multiple serum concentrations and AUC values were reported per treatment arm, they were included separately.

Mean RSDs of vancomycin exposure for Inl and Col were compared by an Independent samples T-test. Statistical analysis were performed using SPSS Statistics version 20 (IBM Corporation, Chicago III, USA, 2011). All tests were performed two-sided and a $p$-value of $<0.05$ was considered statistically significant.

\section{Results}

In total 34 articles were included (see Figure 1 for a flow chart of inclusion).

A summary of study design, dosing regimen and exposure target of the included studies is displayed in Table 1. 
Figure 1 Flow chart of inclusion

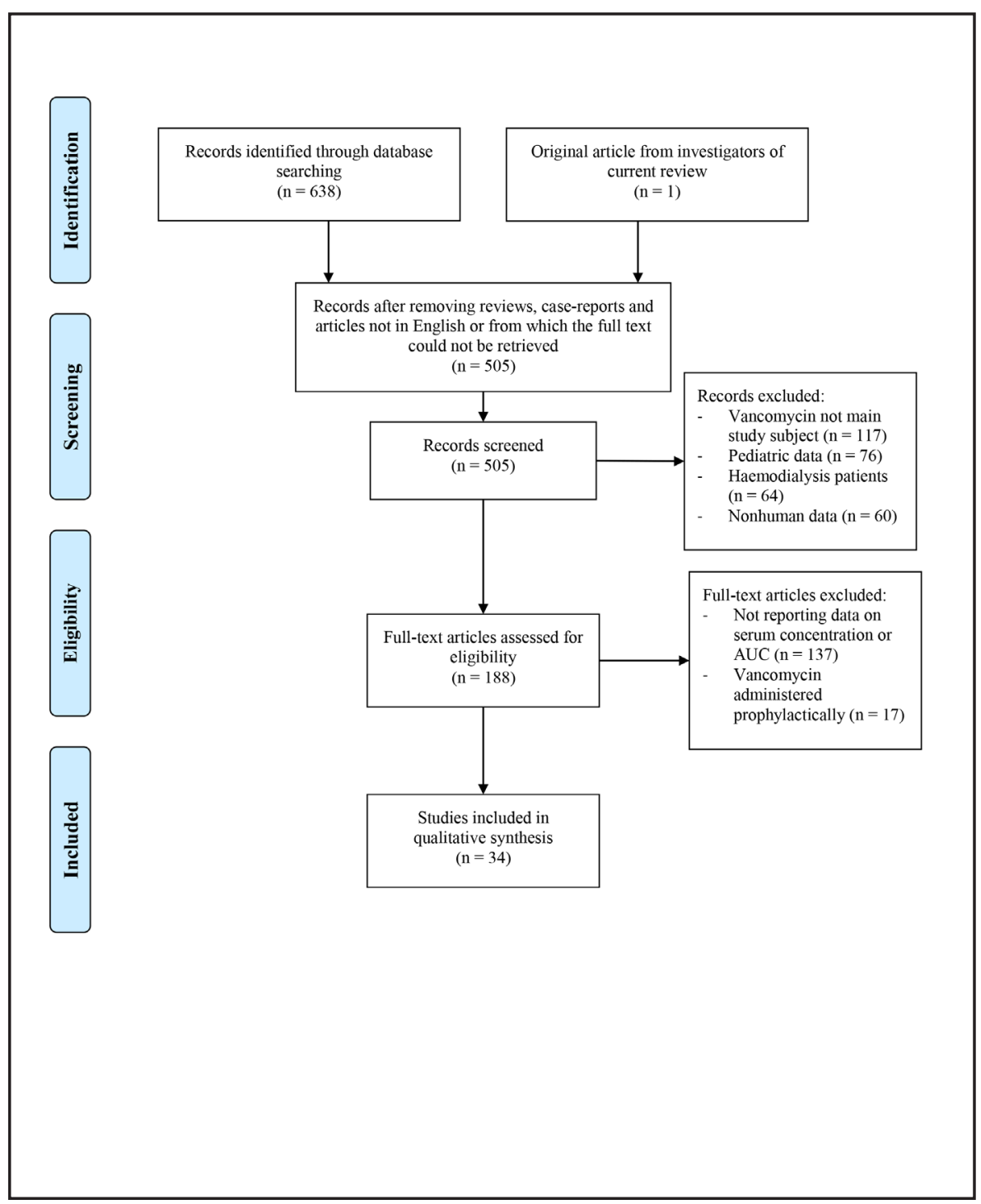




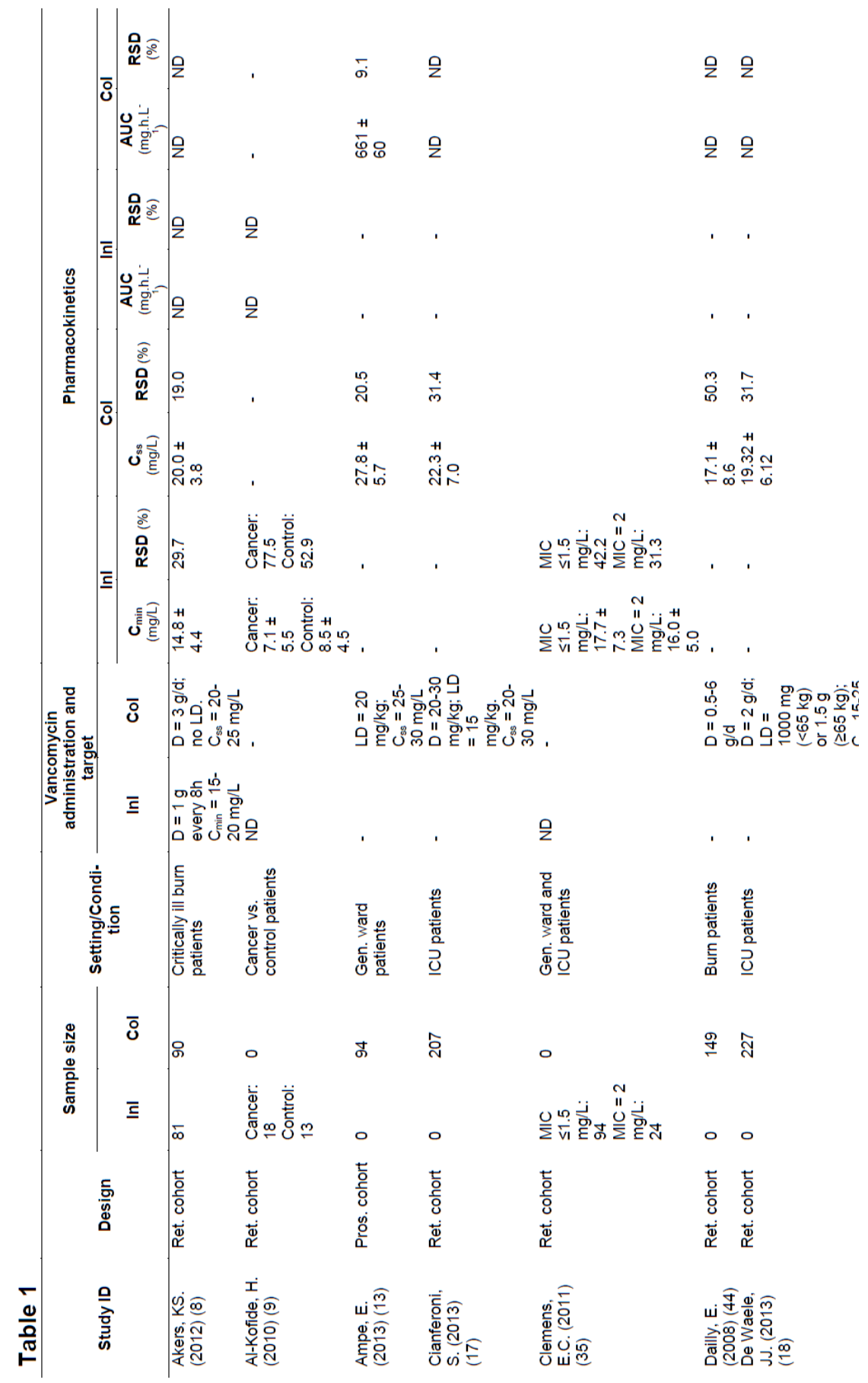




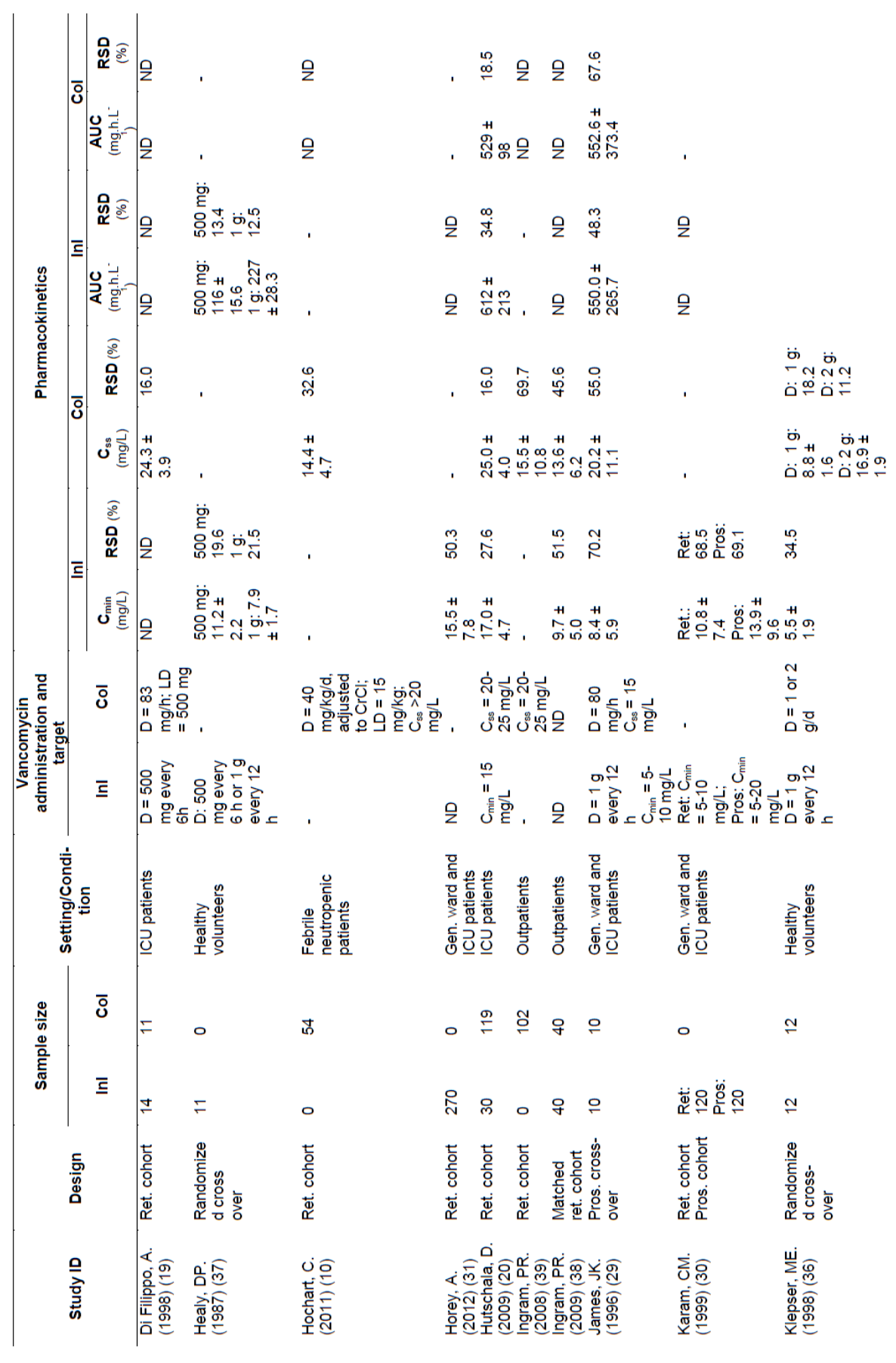




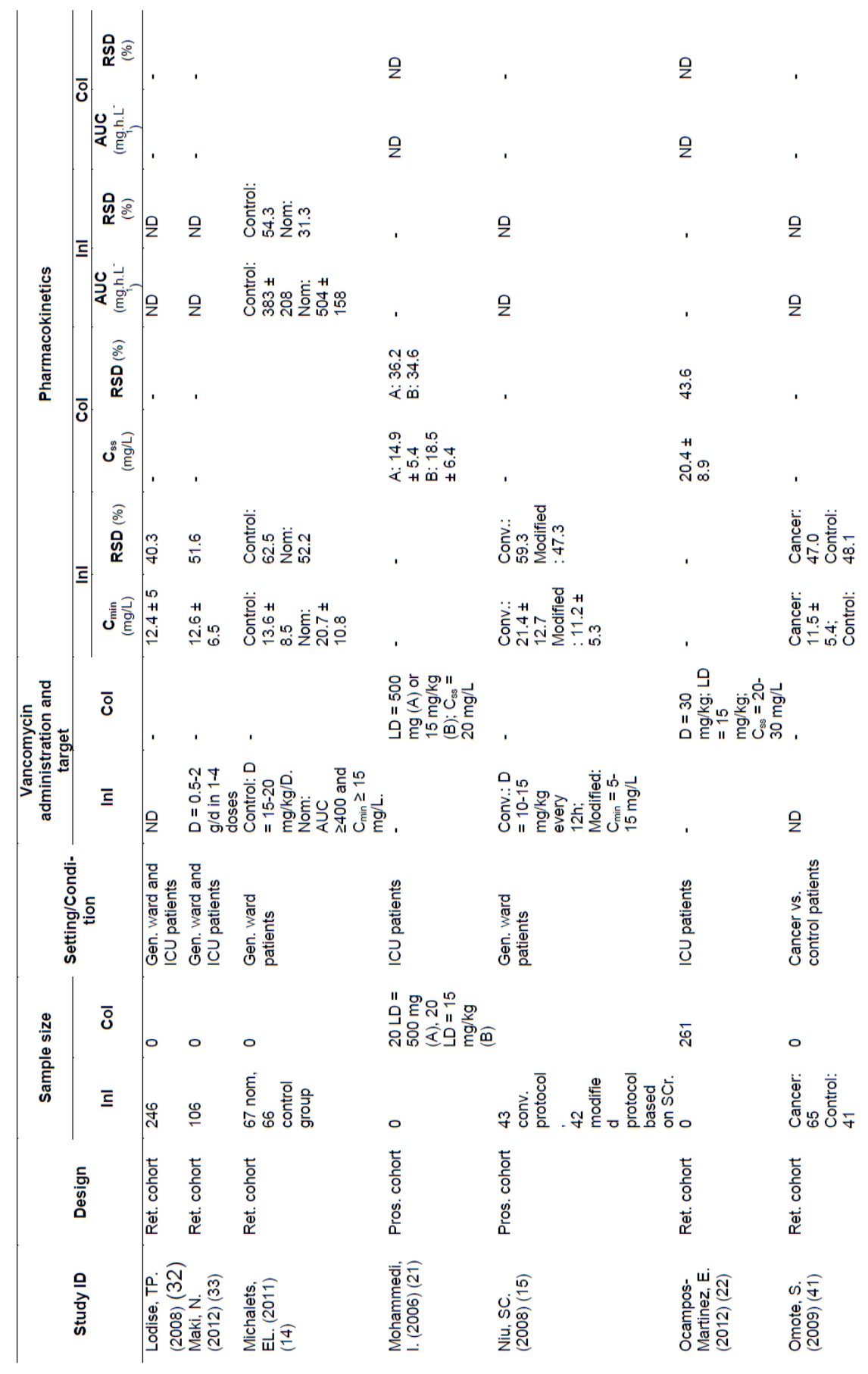




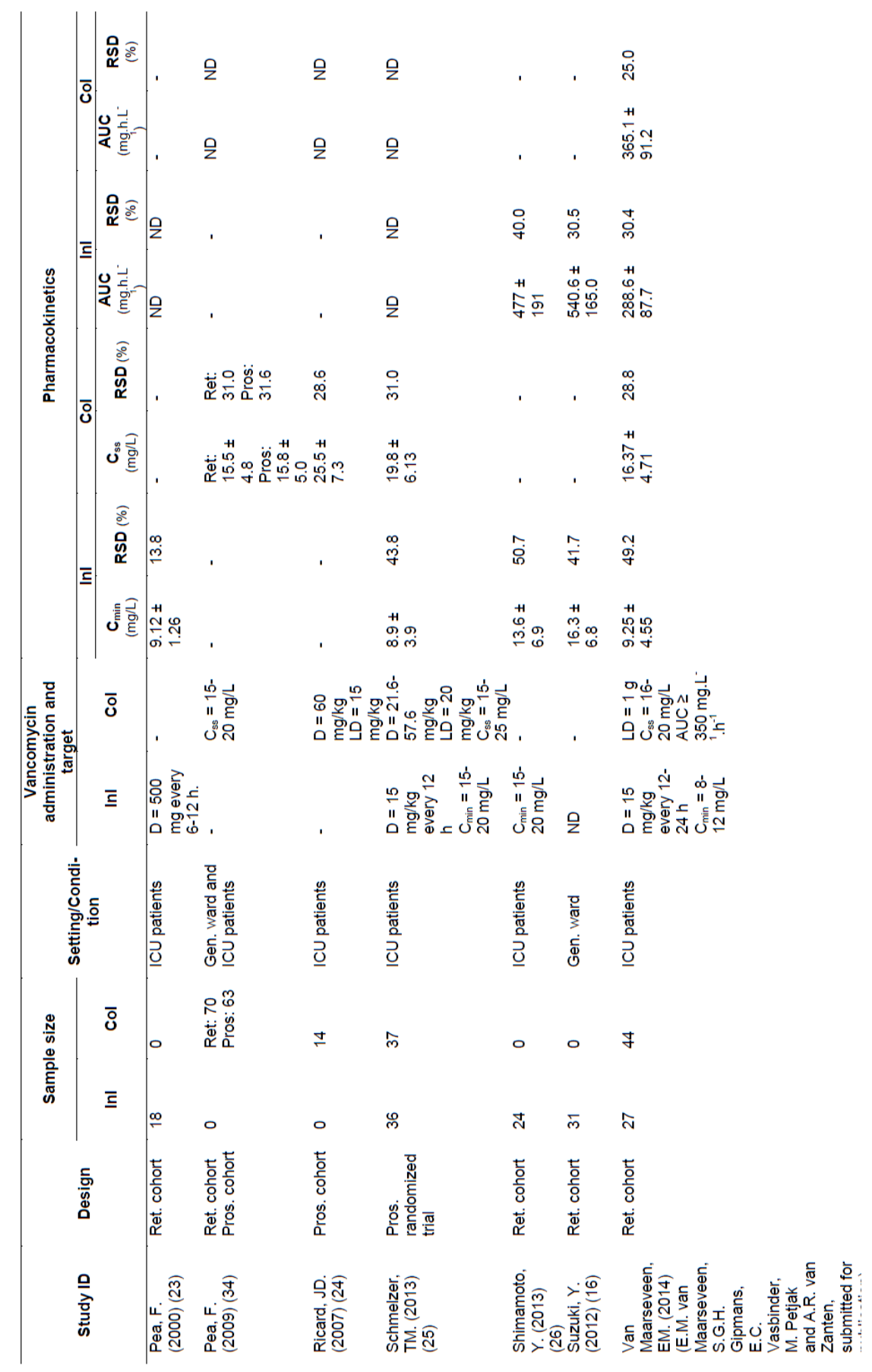




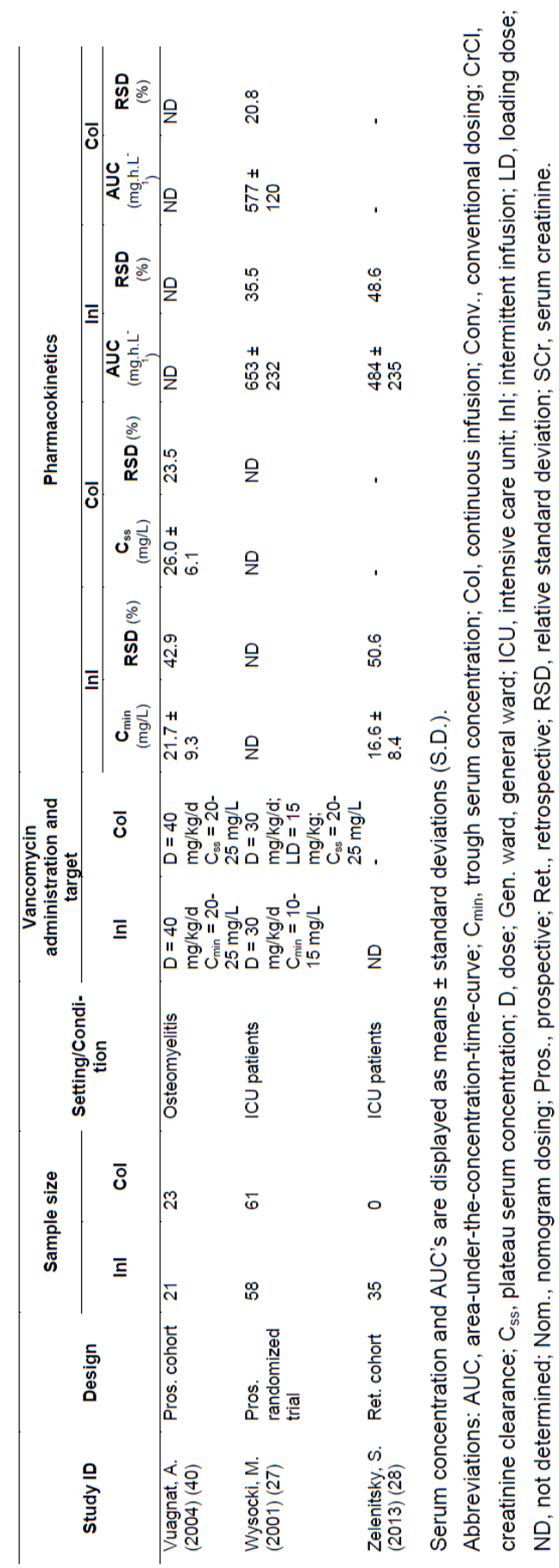




\section{Vancomycin treated populations}

The vancomycin target concentration range depends on the indication and patient population. The consensus guidelines by the American Society of Health-System Pharmacists (ASHP), the Infectious Diseases Society of America (IDSA) and the Society of Infectious Diseases Pharmacists (SIDP) advise to maintain a serum trough concentration of at least $10 \mathrm{mg} / \mathrm{L}$ to prevent development of resistance. More severe infections caused by S. aureus, such as endocarditis, osteomyelitis, meningitis and hospital-acquired pneumonia, may require higher trough concentrations of $15-20 \mathrm{mg} / \mathrm{L}$ to achieve an AUC/MIC $\geq 400$ when the pathogen's MIC $\leq 1 \mathrm{mg} / \mathrm{L}$. A loading dose of $25-30 \mathrm{mg} / \mathrm{kg}$ bodyweight may be considered for critically ill patients to ensure rapid target attainment (4). Since Col is not supported by the IDSA guidelines (4), the targets for this mode of administration are less well defined. However, it may be assumed that the same pharmacokinetic/pharmacodynamic (PK/PD) target of AUC/MIC $\geq 400$ applies to Col, which would require a plateau concentration of $15-25 \mathrm{mg} / \mathrm{L}$ for pathogens with an MIC $\leq 1$ $\mathrm{mg} / \mathrm{L}$ (5). Pathophysiological processes or concomitant therapies may affect vancomycin disposition and clearance and thereby introduce variability of exposure and target attainment. The volume of distribution and vancomycin clearance can vary significantly between populations (7-10). Due to the hydrophilic properties of vancomycin, its volume of distribution is mainly limited to the extracellular fluid content (7). Therefore, any change in this content will influence the serum concentration. Furthermore, vancomycin clearance occurs predominantly through glomerular filtration of the unchanged form (11). Changes in renal function are likely to alter vancomycin clearance and thus exposure. Larger variability in vancomycin concentrations and AUCs can thus be expected in populations with large interpatient variability in volume of distribution and clearance, such as critically ill patients (12). Since exposure variability is largely dependent of the specific population, vancomycin exposure and target attainment rates are reported and discussed in detail per study population: general ward patients, patients admitted to the intensive care unit (ICU), mixed general ward and ICU populations, healthy volunteers, and other populations. 


\section{General ward patients}

Three studies evaluated nomogram dosing in general ward patients; two studies administered vancomycin by Inl and one study by Col (13-15). The Inl studies compared conventional dosing based on body weight to dosing according to a nomogram based on body weight as well as creatinine clearance. Both studies suggest that InI dosing yields better target attainment with a dosing schedule based on body weight and creatinine clearance $(14,15)$. Ampe et al. implemented a nomogram for Col of vancomycin in the general ward and found a variability in serum concentration of $20.5 \%$. Variability in AUC was very low (RSD $9.1 \%$ ), probably because the AUC24h was determined from 48 hours after initiation up to the end of treatment (13). Variability in AUC is expected to be highest in the first 24 hours of treatment, which will be discussed later. To summarize, four studies reported means and standard deviations of serum concentrations or AUCs (mean \pm SD) in general ward patients (13-16). Three studies used Inl as mode of administration and in the remaining study (14-16), patients were treated with Col (13). Overall, variability appeared to be larger in patients that received vancomycin through Inl compared with Col. The median RSDs (range) of trough versus plateau concentrations were $52.2 \%$ (41.7-62.5\%) and $20.5 \%$ (-), for Inl and Col respectively. Only one RSD was available for Col, compared to five for Inl.

\section{ICU patients}

It is widely accepted that patients admitted to the ICU should be considered a pharmacokinetically heterogeneous population (12). Inflammation leads to extravasation of fluids to the interstitial space, thereby increasing the volume of distribution. Furthermore, inotropic drugs can significantly increase cardiac output in critically ill patients. As a result, renal blood flow and thus vancomycin clearance can be enhanced substantially. This condition is known as glomerular hyperfiltration. However, in case of cardial depression, cardiac output may be decreased leading to lower renal blood flow. Acute kidney insufficiency may occur with concomitant decreased vancomycin clearance (12). Thirteen studies reported serum concentrations or AUCs (mean \pm SD) with Inl or Col in ICU patients (17-28) (E.M. van Maarseveen, S.G.H. Gipmans, E.C. Vasbinder, M. Petjak and A.R. van Zanten, submitted for publication). Four of these were direct comparisons of Inl versus Col $(20,25$, 
27) (E.M. van Maarseveen, S.G.H. Gipmans, E.C. Vasbinder, M. Petjak and A.R. van Zanten, submitted for publication). In three studies (23, $26,28)$, Inl was the only mode of infusion used and in the remaining six studies patients were treated through Col $(17-19,21,22,24)$. Only two of these comparative studies in ICU patients were prospective randomized trials. In the most recent one, Schmelzer et al. compared target attainment in the first 48 hours in patients with ventilator-associated pneumonia (25). The target ranges for Inl and Col were $15-20 \mathrm{mg} / \mathrm{L}$ and $15-25 \mathrm{mg} / \mathrm{L}$, respectively. Only $7 \%$ of the patients treated with $\mathrm{InI}$ attained trough concentrations in the target range. An adequate plateau concentration was attained in $57 \%$ of the patients treated with Col $(p<0.0001)$. Furthermore, variability in serum concentrations seemed to be lower with Col (31.0\% versus $43.8 \%$ with $\mathrm{Inl})$ and fewer dose adjustments were necessary with Col compared to Inl $(p=0.002)$. The authors choose to power the study solely as a pharmacokinetic study and reported no clinical outcomes. In the second prospective trial, Wysocki and co-workers showed that variability in AUC and daily dose were lower with Col compared to $\mathrm{Inl}(p=0.026$ and 0.057 , respectively) (27). The authors also reported faster target attainment with Col, which may be clinically relevant in critically ill patients. Targets were attained within $51 \pm 39$ hours and $36 \pm 31$ hours in Inl and Col, respectively $(p=0.03)$. The remaining direct comparisons were retrospective cohort studies. Hutschala et al. confirmed target attainment was reached faster with Col (16 \pm 8 hours) compared to Inl (50 \pm 21 hours; $p<0.001)$ in patients after cardiac surgery $(20)$. The targets were 15 $\mathrm{mg} / \mathrm{L}$ for Inl trough and $20-25 \mathrm{mg} / \mathrm{L}$ for Col plateau concentrations. Van Maarseveen et al. compared variability of exposure in ICU patients treated with Inl to Col treatment (E.M. van Maarseveen, S.G.H. Gipmans, E.C. Vasbinder, M. Petjak and A.R. van Zanten, submitted for publication). Prior to dose adjustment based on TDM, a higher variability in serum concentration (RSD 49.2 and $28.7 \%$ for $\mathrm{Inl}$ and Col, respectively) and AUC (RSD 30.4 and $24.9 \%$ for Inl and Col, respectively) was detected in patients treated with Inl. After dose adjustment based on TDM, the variability in exposure did not change notably for both modes of administration. Furthermore, albeit correlations between serum concentrations and AUCs for both modes of administration were significant $(p<0.01)$, correlation of Col compared to Inl was significantly higher (determinant of correlation ( $r 2$ ) 0.932 and 0.720 , respectively). De Waele et al. sought to explore target attainment, defined as a plateau concentration of $15-25 \mathrm{mg} / \mathrm{L}$, within 48 hours in patients treated 
with Col (18). Target attainment after 48 hours of therapy was $70.5 \%$ and increased to $84.1 \%$ on day 3 . A loading dose of $\geq 15 \mathrm{mg} / \mathrm{kg}$ was significantly associated with attaining adequate vancomycin concentrations on day $2(p=0.006)$. Ocampos-Martinez et al.assigned a target range of $20-30 \mathrm{mg} / \mathrm{L}$ to septic patients on Col vancomycin treatment (22). Adequate concentrations were found in $36 \%$ of the patients on day 1 . This increased to $48 \%$ on day 2 , even though the dose was only adapted in $41 \%$ of the patients with subtherapeutic vancomycin concentrations. On day 3 , target attainment was $68 \%$. Male sex and lower creatinine clearance were risk factors associated with insufficient serum concentrations on day 1 and 2 . Zelenitsky et al. retrospectively collected Inl pharmacokinetic data of patients in septic shock (28). No predefined targets were reported, but the authors observed a main trough concentration $\geq 10 \mathrm{mg} / \mathrm{L}$ in $77.1 \%$ and $\geq 15 \mathrm{mg} / \mathrm{L}$ in $48.6 \%$ of the patients. In $60 \%$ of the patients, AUC was greater than 400 mg.h.L-1. In conclusion, a relatively high variability in exposure is reported in this subset of patients due to a relatively large interpatient variability in PK parameters. Nevertheless, the relative difference between the median RSDs for Inl trough concentrations and Col plateau concentrations were similar to other populations included in this review: $46.5 \%$ (range $13.8-50.7 \%$ ) and $31.2 \%$ (range $16.0-43.6 \%$ ), respectively. These findings are based on ten RSDs for Col and six for Inl.

\section{Mixed General ward and ICU patient population}

James et al. conducted a prospective randomized cross-over study in which vancomycin was administered through Inl or Col for two consecutive days in 10 patients (29). Even though variability in serum concentration appeared to be lower with $\mathrm{Col}(\mathrm{RSD}=55.0 \%$ with Col and RSD $=70.2 \%$ with Inl), variability in AUC appeared to be higher (RSD $=67.6 \%$ and $48.3 \%$ with Col and Inl, respectively). Of note, this is the only study directly comparing Inl and Col in which variability in either serum concentration or AUC appeared to be higher in Col than Inl. Only one study assessed target attainment. Karam et al. conducted a study in order to minimize monitoring and drug adjustments with InI vancomycin (30). Nomogram dosing led to dose adjustments in $20.3 \%$ of the patients and predefined target exposure was attained in $94 \%$. However, a wide target range of $5-20 \mathrm{mg} / \mathrm{L}$ was chosen compared to other studies included in this review. To conclude, seven studies made no distinction between general ward and ICU patients and studied 
vancomycin in hospitalized patients (29-35). As may be expected from such heterogeneous populations, variability in serum concentration was high in this subset of patients (median RSD 31.6, range 31.0-55.0 for Col based on three RSDs and median RSD 51.0, range 31.3-70.2 for Inl based on eight RSDs).

\section{Healthy volunteers}

Klepser et al. conducted a randomised, open-label, cross-over trial in which volunteers received a three day course of $\mathrm{Inl}(1000 \mathrm{mg} /$ day $)$ or Col (1000 or $2000 \mathrm{mg} /$ day) vancomycin (36). Samples were obtained after two days of treatment. Variability in serum concentration was higher with Inl (RSD = 34.5\%) compared to Col (RSD = 18.2 and $11.2 \%$ for $1000 \mathrm{mg} /$ day and $2000 \mathrm{mg} /$ day, respectively). Thrombophlebitis was a frequently reported complaint, mainly in patients treated with Col. This was due to infusion via a peripheral vein as opposed to a central vein. Healy et al. compared Inl twice daily to four times a day in a randomised cross-over study with eleven healthy volunteers (37). The variability in trough concentrations (RSD $19.6 \%$ for four times a day and $21.5 \%$ for twice daily) and AUC (RSD $13.4 \%$ and $12.5 \%$ for four and two times a day, respectively) were comparable between both groups. The variation in serum concentrations could mainly be explained by body weight according to the authors. In conclusion, variability of exposure in healthy volunteers was relatively low, probably due to strict inclusion and exclusion criteria, resulting in a population with limited variability in PK parameters. Two studies were conducted in healthy volunteers, yielding two RSD's for Col and three for Inl $(36,37)$. Median (range) RSD's of $14.7 \%$ (11.2-18.2\%) and $21.5 \%$ (19.6-34.5\%) for serum concentrations were acquired with Col and Inl, respectively.

\section{Other populations}

The remainder of the studies included burn patients, orthopaedic outpatients, oncologic patients and febrile neutropenic patients. In a retrospective cohort study, Akers et al. evaluated the use of Inl and Col in critically ill burn patients (8). Burn patients are known to have a larger volume of distribution and clearance when compared to non-burn patients. Target trough and plateau concentration were $15-20 \mathrm{mg} / \mathrm{L}$ and $20-25 \mathrm{mg} / \mathrm{L}$, respectively. These targets were attained in $22.6 \%$ of the patients treated with Inl and $30.7 \%$ of the patients treated with Col $(p=0.01)$. Furthermore, variability in serum concentration was larger 
with Inl as opposed to Col (RSD $=29.7$ and $19.0 \%$, respectively). No differences in clinical outcomes were observed. However, the study was underpowered to assess outcomes such as mortality and nephrotoxicity.

Three studies were conducted in orthopaedic outpatients (38-40). It should be mentioned that these patients are at increased risk of suboptimal therapeutic drug monitoring due to inappropriate timed sampling, especially for Inl. Vuagnat et al. conducted a prospective comparative study in patients with osteomyelitis and strived for high vancomycin trough concentrations (trough and plateau level of $20-25 \mathrm{mg} / \mathrm{L}$ ) (40). Treatment was initiated in hospitalized patients who were discharged upon achieving steady-state concentrations. The target serum concentrations were attained after $2.5 \pm 2.8$ weeks for Inl and $1.4 \pm 0.7$ weeks for Col $(p=0.18)$. Variability in serum concentration at steady-state was significantly lower with Col than with $\operatorname{Inl}(p=0.001)$. Ingram et al. conducted two retrospective studies that focused on nephrotoxicity (38, 39). Only one of these studies directly compared Inl or Col and yielded comparable variability in serum concentration $(\mathrm{RSD}=51.5 \%$ for $\mathrm{Inl}$ and $45.6 \%$ for Col) (38).

Two retrospective cohort studies compared vancomycin pharmacokinetics in cancer patients to non-cancer patients $(9,41)$. Al-Kofide et al. found that the volume of distribution and vancomycin clearance were higher in cancer patients ( $p=0.002$ and $p=0.005$, respectively) (9). This resulted in significantly higher peak but not trough concentrations in the control patients compared to the cancer patients $(p=0.027$ and $p=0.458$, respectively). Furthermore, variability in trough concentration was higher in cancer patients compared to controls (RSD $=77.5 \%$ versus $52.9 \%$, respectively). In contrast, Omote et al. did not report enhanced vancomycin clearance in cancer patients when compared to control patients (41). Variability in serum concentration was comparable between the two groups (RSD 47.0 and $48.1 \%$ for cancer and control patients, respectively).

Hochart et al. conducted a retrospective cohort study in patients with febrile neutropenia to assess the attainment of adequate initial or follow-up serum concentrations (10). The volume of distribution is often increased in these patients. Vancomycin was administered through $\mathrm{Col}$ and the target concentration was $20 \mathrm{mg} / \mathrm{L}$. This target was initially attained in $12 \%$ of the patients. In only $40 \%$ of the cases, serum monitoring was performed optimally. Eventually $32 \%$ had a plateau serum concentration of $\geq 20 \mathrm{mg} / \mathrm{L}$ after dose adjustment. 


\section{Overall comparison}

The patient populations in the included studies were very heterogeneous: two studies were conducted in healthy volunteers; four in general ward patients; thirteen studies were performed in ICU patients and seven studies were performed in hospitalized patients (general ward and ICU). Furthermore, eight studies were conducted in specific populations such as outpatients, which were mainly orthopaedic patients; cancer versus non-cancer patients; febrile neutropenia and burn patients. Variability in serum concentration, expressed as RSD, ranged from 11.2 to $77.5 \%$ with a mean of $40.3 \%$. Overall, variability in serum concentration as well as AUC appeared to be higher in vancomycin Inl treated patients compared to Col treated patients. The mean RSDs in serum concentration were $46.5 \%$ (15.4\%) and $32.1 \%$ (14.1\%) for Inl and Col, respectively and differed significantly between both treatment groups $(p=0.001)$ (Figure 2$)$. 
Figure 2. Boxplots of the relative standard deviations of serum concentration with intermittent or continuous infusion of vancomycin.

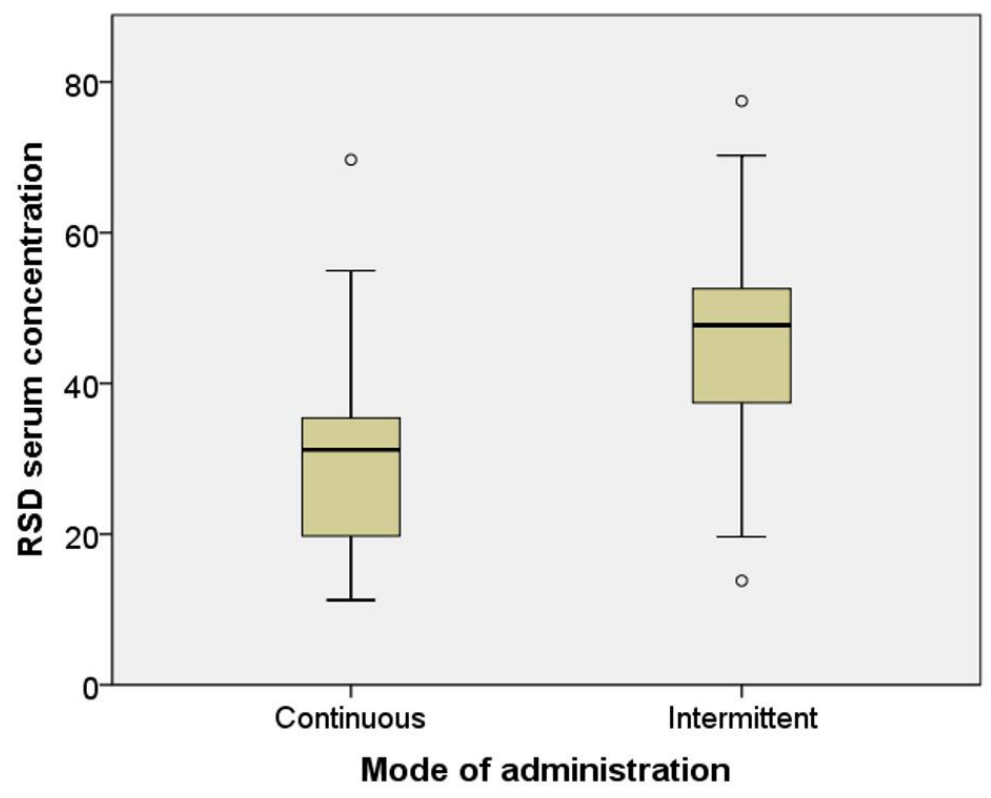

The influence of study design and number of patients included in the study was explored and reported in table 2. 
Table 2. Distribution of relative standard deviations (RSDs) of serum concentrations in different study designs

\begin{tabular}{lllll}
\hline $\begin{array}{l}\text { Mode of } \\
\text { administration }\end{array}$ & Study design & $\begin{array}{l}\text { No. of RSDs } \\
(\mathrm{n})\end{array}$ & $\begin{array}{l}\text { No. patients } \\
\text { per study }(\mathrm{n}), \\
\text { median }\end{array}$ & $\begin{array}{l}\text { RSD (\%) } \\
\text { mean } \pm \text { SD }\end{array}$ \\
\hline Inl & Total & 30 & 38 & $46.5 \pm 15.4$ \\
& Ret. cohort & 20 & 41 & $47.0 \pm 14.3$ \\
& Pros. cohort & 4 & 43 & $54.6 \pm 11.9$ \\
& Clinical trial & 6 & 12 & $38.0 \pm 20.6$ \\
\hline Col & Total & 23 & 54 & $32.1 \pm 14.1$ \\
& Ret. cohort & 12 & 96 & $34.6 \pm 15.6$ \\
& Pros. cohort & 6 & 22 & $29.2 \pm 6.2$ \\
& Clinical trial & 5 & 12 & $28.8 \pm 19.2$ \\
\hline
\end{tabular}

Abbreviations are Col, continuous infusion; Inl, intermittent infusion; Pros, prospective; Ret, retrospective; RSD, relative standard deviation; SD, standard deviation.

\section{Discussion and Conclusion}

Differences in exposure variability between Inl and Col treatment can largely be explained by the pharmacokinetic profile of vancomycin, which is best described by a two compartment model. After infusion the distribution phase lasts 30-60 minutes, after which equilibrium between the central and the peripheral compartment is reached $(11,42)$. The vancomycin peak concentration is determined after the distribution phase, in general one to two hours after infusion. The vast majority of vancomycin is cleared through renal excretion, more specifically through glomerular filtration. The degree of clearance thus depends on the glomerular filtration rate, which is estimated in clinical practice by the creatinine clearance (11). Under steady-state conditions with Inl, $\Delta$ Cpeak-Ctrough is dependent of the dose and volume of distribution as described by equation 1

Cpeak-Ctrough $=\mathrm{D} / \mathrm{Vd}($ equation 1$)$ 
where $\mathrm{D}$ and $\mathrm{Vd}$ represent the dose and volume of distribution, respectively. Consequently, a relatively high interpatient variability in volume of distribution will result in a high variation in trough levels between individual patients. Besides the volume of distribution, trough concentrations are affected by interpatient variability in clearance. For Col, clearance is the pharmacokinetic parameter that is correlated with Css as defined by equation 2 .

Css $=\operatorname{Rinf} / C l$ (equation 2)

where Css, Rinf and $\mathrm{Cl}$ are the concentration in steady-state conditions, the rate of infusion and clearance, respectively. Furthermore, the timing of sampling is of great importance when obtaining trough serum concentrations (figure 3), especially since current IDSA guidelines indicate trough concentrations should be used to monitor efficacy of vancomycin treatment (4).

Vancomycin serum concentrations not drawn directly before the next administration require extrapolation to a true trough serum concentration, which introduces uncertainty and increases variability. In patients on Col, on the other hand, the timing of samplinig is of less importance. Plateau concentrations depend on the infusion rate and clearance and therefore variability is caused by variation in clearance only (equation 2). Finally, the variation in duration of the distribution phase in patients on vancomycin Inl will not affect trough concentrations, but can hurdle accurate and precise estimation of AUC values derived from limited sampling strategies. In patients on vancomycin Col, on the other hand, this effect will be marginal or even absent under absolute steady state condition (43). This is eminent; if in the future vancomycin dosing guided by AUC targets would be preferred.

Independent of the mode of administration, the highest variability of exposure can be expected during the first 24 hours when steady-state conditions are not yet achieved as can be seen in figure 3. Importantly, it has been shown that the correlation between serum concentrations and estimated AUCs is greater in Col compared to Inl, rendering plateau concentrations better predictors of vancomycin exposure in terms of AUC (E.M. van Maarseveen, S.G.H. Gipmans, E.C. Vasbinder, M. Petjak and A.R. van Zanten, submitted for publication). 
Figure 3. Simulated pharmacokinetic curves of patients treated with intermittent (Inl) or continuous infusion (Col) of vancomycin.

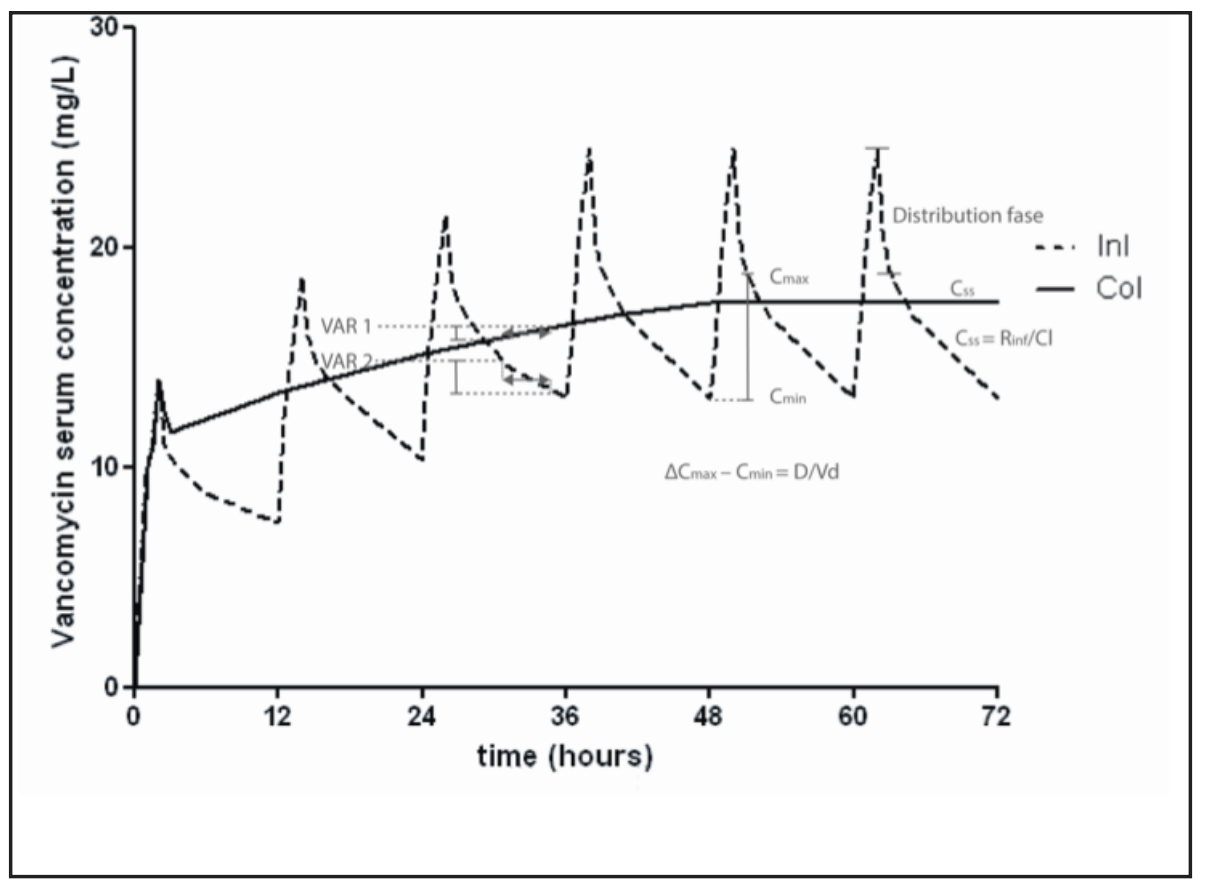

The interval between peak (Cmax) and trough (Cmin) serum concentration in patients treated with vancomycin Inl (dashed line) is described by the dose (D) divided by the volume of distribution (Vd). C $\neg$ max is determined after the distribution phase. The plateau serum concentration (Css) in patients treated with vancomycin Col (continuous line) is described by the infusion rate of vancomycin (Rinf) divided by the clearance (CI). VAR 1 and VAR 2 illustrate the variability in serum concentration caused by a shift of sampling time. 
Studies comparing clinical outcome measures of vancomycin Inl and Col treated patients are often underpowered. In this review, an alternate strategy by investigating differences in the variability of exposure between Col and InI was performed. We found that the variability in vancomycin concentrations is larger for treatment with Inl vancomycin compared to Col. A 50\% greater RSD in serum concentration was detected for Inl compared to Col. It is important to note that a $50 \%$ larger RSD results in a 2 times larger 95\%-confidence interval of trough versus plateau concentrations. Furthermore, the studies that directly compared target attainment of $\mathrm{Inl}$ and Col found more rapid and higher target attainment with Col $(20,25,27,38)$. Higher variability of exposure with Inl compared to Col can be largely explained by the effect of variations in volume of distribution and sample timing. It remains to be elucidated whether the lower variability of exposure and better target attainment associated with Col can improve clinical outcomes. To the best of our knowledge, this is the first systematic review to address exposure variability in Inl and Col vancomycin treatment. Variability in serum concentration may be an important factor in achieving rapid target attainment, which is of critical importance in the treatment of critically ill patients. There are several limitations to this review. First of all, the majority of included RSDs were derived from retrospective cohort studies (32 out of 53 RSDs). Even though this may introduce more variability due to lower protocol adherence, it does represent data obtained in clinical practice. Secondly the heterogeneity of the patient population included could be considered a limitation, since targets and dosing protocols are known to differ between centres. However, in our analysis the relative and not the absolute serum concentration variability was examined, correcting for the different target concentrations in InI and Col treated populations. Finally, systematic reviews are at risk of selection bias. Nevertheless, to our best knowledge all studies reporting vancomycin serum concentration variability in adult patients not on renal replacement therapy were included in this review. Moreover, analysing the data stratified by patient population or methodological design showed that the variability in Col treated patients was lower in relation to Inl treated patients in all reported comparisons.

Finally, the definition of target attainment varied largely between the included studies, which hampered the analysis of this outcome measure. 


\section{References}

1. Moellering, R. C.,Jr. 2006. Vancomycin: a 50-year reassessment. Clin. Infect. Dis. 42 Suppl 1:S3-4. doi: 10.1086/491708.

2. Elyasi, S., H. Khalili, S. Dashti-Khavidaki, and A. Mohammadpour. 2012. Vancomycin-induced nephrotoxicity: mechanism, incidence, risk factors and special populations. A literature review. Eur. J. Clin. Pharmacol. 68:1243-1255. doi: 10.1007/s00228-0121259-9; 10.1007/s00228-012-1259-9.

3. Levine, D. P. 2006. Vancomycin: a history. Clin. Infect. Dis. 42 Suppl 1:S5-12. doi: 10.1086/491709.

4. Rybak, M., B. Lomaestro, J. C. Rotschafer, R. Moellering Jr, W. Craig, M. Billeter, J. R. Dalovisio, and D. P. Levine. 2009. Therapeutic monitoring of vancomycin in adult patients: a consensus review of the American Society of Health-System Pharmacists, the Infectious Diseases Society of America, and the Society of Infectious Diseases Pharmacists. Am. J. Health. Syst. Pharm. 66:82-98. doi: 10.2146/ajhp080434; 10.2146/ ajhp080434.

5. Smuszkiewicz, P., E. Szalek, H. Tomczak, and E. Grzeskowiak. 2013. Continuous infusion of antibiotics in critically ill patients. Curr. Clin. Pharmacol. 8:13-24.

6. Cataldo, M. A., E. Tacconelli, E. Grilli, F. Pea, and N. Petrosillo. 2012. Continuous versus intermittent infusion of vancomycin for the treatment of Gram-positive infections: systematic review and meta-analysis. J. Antimicrob. Chemother. 67:17-24. doi: 10.1093/jac/dkr442; 10.1093/jac/dkr442.

7. Pea, F., P. Viale, and M. Furlanut. 2005. Antimicrobial therapy in critically ill patients: a review of pathophysiological conditions responsible for altered disposition and pharmacokinetic variability. Clin. Pharmacokinet. 44:1009-1034. doi: 10.2165/00003088200544100-00002.

8. Akers, K. S., J. M. Cota, K. K. Chung, E. M. Renz, K. Mende, and C. K. Murray. 2012. Serum vancomycin levels resulting from continuous or intermittent infusion in critically ill burn patients with or without continuous renal replacement therapy. J. Burn Care. Res. 33:e254-62. doi: 10.1097/BCR.0b013e31825042fa; 10.1097/BCR.0b013e$31825042 \mathrm{fa}$.

9. Al-Kofide, H., I. Zaghloul, and L. Al-Naim. 2010. Pharmacokinetics of vancomycin in adult cancer patients. J. Oncol. Pharm. Pract. 16:245-250. doi:

$10.1177 / 1078155209355847 ; 10.1177 / 1078155209355847$.

10. Hochart, C., C. Berthon, S. Corm, J. Gay, M. Cliquennois, S. Tricot, and S. Alfandari. 2011. Vancomycin serum concentration during febrile neutropenia in patients with acute myeloid leukemia. Med. Mal. Infect. 41:652-656. doi: 10.1016/j.medmal.2011.09.014; 10.1016/j.medmal.2011.09.014.

11. Rybak, M. J. 2006. The pharmacokinetic and pharmacodynamic properties of vancomycin. Clin. Infect. Dis. 42 Suppl 1:S35-9. doi: 10.1086/491712.

12. Roberts, J. A., and J. Lipman. 2009. Pharmacokinetic issues for antibiotics in the critically ill patient. Crit. Care Med. 37:840-51; quiz 859. doi: 10.1097/CCM. 0b013e3181961bff; 10.1097/CCM.0b013e3181961bff.

13. Ampe, E., B. Delaere, J. D. Hecq, P. M. Tulkens, and Y. Glupczynski. 2013. Implementation of a protocol for administration of vancomycin by continuous infusion: pharmacokinetic, pharmacodynamic and toxicological aspects. Int. J. Antimicrob. Agents. 41:439-446. doi: 10.1016/j.ijantimicag.2013.01.009; 10.1016/j.ijantimicag.2013.01.009. 14. Michalets, E. L., C. L. Pounders, S. J. Hollis, and S. Sutherland. 2011. Outcomes 
associated with AUC24/MIC nomogram dosing of vancomycin. Ann. Pharmacother. 45:687-689. doi: 10.1345/aph.1P495; 10.1345/aph.1P495.

15. Niu, S. C., S. T. Deng, M. H. Lee, C. Ho, H. Y. Chang, and F. H. Liu. 2008. Modified vancomycin dosing protocol for treatment of diabetic foot infections. Am. J. Health.

Syst. Pharm. 65:1740-1743. doi: 10.2146/ajhp080004; 10.2146/ajhp080004.

16. Suzuki, Y., K. Kawasaki, Y. Sato, I. Tokimatsu, H. Itoh, K. Hiramatsu, M. Takeyama, and J. Kadota. 2012. Is peak concentration needed in therapeutic drug monitoring of vancomycin? A pharmacokinetic-pharmacodynamic analysis in patients with methicillin-resistant staphylococcus aureus pneumonia. Chemotherapy. 58:308-312. doi: 10.1159/000343162; 10.1159/000343162.

17. Cianferoni, S., A. Devigili, E. Ocampos-Martinez, L. Penaccini, S. Scolletta, A. Abdelhadii, D. De Backer, M. Beumier, F. Jacobs, J. L. Vincent, and F. S. Taccone. 2013. Development of acute kidney injury during continuous infusion of vancomycin in septic patients. Infection. 41:811-820. doi: 10.1007/s15010-013-0460-9; 10.1007/s15010-0130460-9.

18. De Waele, J. J., I. Danneels, P. Depuydt, J. Decruyenaere, M. Bourgeois, and E. Hoste. 2013. Factors associated with inadequate early vancomycin levels in critically ill patients treated with continuous infusion. Int. J. Antimicrob. Agents. 41:434-438. doi: 10.1016/j.ijantimicag.2012.12.015; 10.1016/j.ijantimicag.2012.12.015.

19. Di Filippo, A., A. R. De Gaudio, A. Novelli, E. Paternostro, C. Pelagatti, P. Livi, and G. P. Novelli. 1998. Continuous infusion of vancomycin in methicillin-resistant staphylococcus infection. Chemotherapy. 44:63-68.

20. Hutschala, D., C. Kinstner, K. Skhirdladze, F. Thalhammer, M. Muller, and E.

Tschernko. 2009. Influence of vancomycin on renal function in critically ill patients after cardiac surgery: continuous versus intermittent infusion. Anesthesiology. 111:356-365. doi: 10.1097/ALN.0b013e3181a97272; 10.1097/ALN.0b013e3181a97272.

21. Mohammedi, I., E. Descloux, L. Argaud, J. Le Scanff, and D. Robert. 2006. Loading dose of vancomycin in critically ill patients: $15 \mathrm{mg} / \mathrm{kg}$ is a better choice than $500 \mathrm{mg}$. Int. J. Antimicrob. Agents. 27:259-262. doi: 10.1016/j.ijantimicag.2005.11.009.

22. Ocampos-Martinez, E., L. Penaccini, S. Scolletta, A. Abdelhadii, A. Devigili, S. Cianferoni, D. de Backer, F. Jacobs, F. Cotton, J. L. Vincent, and F. S. Taccone. 2012. Determinants of early inadequate vancomycin concentrations during continuous infusion in septic patients. Int. J. Antimicrob. Agents. 39:332-337. doi: 10.1016/j.ijantimicag.2011.12.008; 10.1016/j.ijantimicag.2011.12.008.

23. Pea, F., L. Porreca, M. Baraldo, and M. Furlanut. 2000. High vancomycin dosage regimens required by intensive care unit patients cotreated with drugs to improve haemodynamics following cardiac surgical procedures. J. Antimicrob. Chemother. 45:329335.

24. Ricard, J. D., M. Wolff, J. C. Lacherade, B. Mourvillier, N. Hidri, G. Barnaud, G. Chevrel, L. Bouadma, and D. Dreyfuss. 2007. Levels of vancomycin in cerebrospinal fluid of adult patients receiving adjunctive corticosteroids to treat pneumococcal meningitis: a prospective multicenter observational study. Clin. Infect. Dis. 44:250-255. doi: 10.1086/510390.

25. Schmelzer, T. M., A. B. Christmas, H. J. Norton, B. T. Heniford, and R. F. Sing. 2013. Vancomycin Intermittent Dosing versus Continuous Infusion for Treatment of Ventilator-associated Pneumonia in Trauma Patients. Am. Surg. 79:1185-1190. 26. Shimamoto, Y., T. Fukuda, K. Tanaka, K. Komori, and D. Sadamitsu. 2013. Systemic inflammatory response syndrome criteria and vancomycin dose requirement in 
patients with sepsis. Intensive Care Med. 39:1247-1252. doi: 10.1007/s00134-0132909-9; 10.1007/s00134-013-2909-9.

27. Wysocki, M., F. Delatour, F. Faurisson, A. Rauss, Y. Pean, B. Misset, F. Thomas, J. F. Timsit, T. Similowski, H. Mentec, L. Mier, and D. Dreyfuss. 2001. Continuous versus intermittent infusion of vancomycin in severe Staphylococcal infections: prospective multicenter randomized study. Antimicrob. Agents Chemother. 45:2460-2467.

28. Zelenitsky, S., E. Rubinstein, R. Ariano, H. lacovides, P. Dodek, Y. Mirzanejad, A. Kumar, and Cooperative Antimicrobial Therapy of Septic Shock-CATSS Database Research Group. 2013. Vancomycin pharmacodynamics and survival in patients with methicillin-resistant Staphylococcus aureus-associated septic shock. Int. J. Antimicrob. Agents. 41:255-260. doi: 10.1016/j.ijantimicag.2012.10.015; 10.1016/j.ijantimicag.2012.10.015.

29. James, J. K., S. M. Palmer, D. P. Levine, and M. J. Rybak. 1996. Comparison of conventional dosing versus continuous-infusion vancomycin therapy for patients with suspected or documented gram-positive infections. Antimicrob. Agents Chemother. 40:696-700.

30. Karam, C. M., P. S. McKinnon, M. M. Neuhauser, and M. J. Rybak. 1999. Outcome assessment of minimizing vancomycin monitoring and dosing adjustments. Pharmacotherapy. 19:257-266.

31. Horey, A., K. A. Mergenhagen, and A. Mattappallil. 2012. The Relationship of nephrotoxicity to vancomycin trough serum concentrations in a veteran's population: a retrospective analysis. Ann. Pharmacother. 46:1477-1483. doi: 10.1345/aph.1R158; 10.1345/aph.1R158.

32. Lodise, T. P., B. Lomaestro, J. Graves, and G. L. Drusano. 2008. Larger vancomycin doses (at least four grams per day) are associated with an increased incidence of nephrotoxicity. Antimicrob. Agents Chemother. 52:1330-1336. doi: 10.1128/AAC.0160207; 10.1128/AAC.01602-07.

33. Maki, N., A. Ohkuchi, Y. Tashiro, M. R. Kim, M. Le, T. Sakamoto, S. Matsubara, and Y. Hakamata. 2012. Initial dose of vancomycin based on body weight and creatinine clearance to minimize inadequate trough levels in Japanese adults. Eur. J. Clin. Microbiol. Infect. Dis. 31:2537-2543. doi: 10.1007/s10096-012-1593-y.

34. Pea, F., M. Furlanut, C. Negri, F. Pavan, M. Crapis, F. Cristini, and P. Viale. 2009. Prospectively validated dosing nomograms for maximizing the pharmacodynamics of vancomycin administered by continuous infusion in critically ill patients. Antimicrob. Agents Chemother. 53:1863-1867. doi: 10.1128/AAC.01149-08; 10.1128/AAC.0114908.

35. Clemens, E. C., J. D. Chan, J. B. Lynch, and T. H. Dellit. 2011. Relationships between vancomycin minimum inhibitory concentration, dosing strategies, and outcomes in methicillin-resistant Staphylococcus aureus bacteremia. Diagn. Microbiol. Infect. Dis. 71:408-414. doi: 10.1016/j.diagmicrobio.2011.08.002; 10.1016/j.diagmicrobio.2011.08.002.

36. Klepser, M. E., K. B. Patel, D. P. Nicolau, R. Quintiliani, and C. H. Nightingale. 1998. Comparison of bactericidal activities of intermittent and continuous infusion dosing of vancomycin against methicillin-resistant Staphylococcus aureus and Enterococcus faecalis. Pharmacotherapy. 18:1069-1074.

37. Healy, D. P., R. E. Polk, M. L. Garson, D. T. Rock, and T. J. Comstock. 1987. Comparison of steady-state pharmacokinetics of two dosage regimens of vancomycin in normal volunteers. Antimicrob. Agents Chemother. 31:393-397. 
38. Ingram, P. R., D. C. Lye, D. A. Fisher, W. P. Goh, and V. H. Tam. 2009. Nephrotoxicity of continuous versus intermittent infusion of vancomycin in outpatient parenteral antimicrobial therapy. Int. J. Antimicrob. Agents. 34:570-574. doi: 10.1016/j.ijantimicag.2009.07.011; 10.1016/j.jantimicag.2009.07.011.

39. Ingram, P. R., D. C. Lye, P. A. Tambyah, W. P. Goh, V. H. Tam, and D. A. Fisher. 2008. Risk factors for nephrotoxicity associated with continuous vancomycin infusion in outpatient parenteral antibiotic therapy. J. Antimicrob. Chemother. 62:168-171. doi: 10.1093/jac/dkn080; 10.1093/jac/dkn080.

40. Vuagnat, A., R. Stern, A. Lotthe, H. Schuhmacher, M. Duong, P. Hoffmeyer, and L. Bernard. 2004. High dose vancomycin for osteomyelitis: continuous vs. intermittent infusion. J. Clin. Pharm. Ther. 29:351-357. doi: 10.1111/j.1365-2710.2004.00572.x.

41. Omote, S., Y. Yano, T. Hashida, S. Masuda, I. Yano, T. Katsura, and K. Inui. 2009. A retrospective analysis of vancomycin pharmacokinetics in Japanese cancer and non-cancer patients based on routine trough monitoring data. Biol. Pharm. Bull. 32:99104.

42. Scaglione, F., and L. Paraboni. 2008. Pharmacokinetics/pharmacodynamics of antibacterials in the Intensive Care Unit: setting appropriate dosing regimens. Int. J. Antimicrob. Agents. 32:294-301. doi: 10.1016/j.ijantimicag.2008.03.015; 10.1016/j. ijantimicag.2008.03.015.

43. Rowland, M., and T.N. Tozer. 1995. Clinical pharmacokinetics. Lippincott Williams \& Wilkins, Phyladelphia, Pennsylvania, USA.

44. Dailly, E., R. Le Floch, G. Deslandes, M. Pannier, and P. Jolliet. 2008. Influence of glomerular filtration rate on the clearance of vancomycin administered by continuous infusion in burn patients. Int. J. Antimicrob. Agents. 31:537-539. doi: 10.1016/j.ijantimicag.2008.02.008; 10.1016/j.jjantimicag.2008.02.008. 


\title{
Chapter 3.2
}

Exposure variability and target attainment of vancomycin intermittent and continuous infusion in critically ill patients -ready, aim, target locked?

\author{
ERIK M. VAN MAARSEVEEN \\ SUZAN GIPMANS \\ ERWIN VASBINDER \\ MANFRED PETJAK \\ ARTHUR R.H. VAN ZANTEN
}

submitted 


\begin{abstract}
Switching from intermittent to continuous infusion has been proposed to increase target attainment rates. Therefore, we investigated the exposure variability in critically ill patients on vancomycin infusion. A retrospective cohort study was conducted among critically ill patients admitted to a level 2 intensive care unit between April 2007 and November 2013. Vancomycin was administered through intravenous infusion and dosing was guided by therapeutic drug monitoring. In 2010 vancomycin administered was switched from intermittent to continous infusion. Results showed the serum concentration variability was twice as high in intermittently dosed population compared to continuously infused patients. Importantly, concentration and area-under-the-curve correlations were stronger in patients on vancomycin continuous infusion. In conclusion, switching from intermittent to vancomycin continuous infusion can lower exposure variability and may thereby improve clinical outcomes in critically ill patients.
\end{abstract}

\title{
Introduction
}

The glycopeptide vancomycin has a time- or area-under-the-concentration-time-curve (AUC)-dependent bacterial killing profile. Based on in vitro and in vivo studies the area-under-the-concentration-time-curve over the minimal inhibitory concentration (AUC/MIC) is considered to be an important predictor of efficacy (1). In critically ill patients large variability in vancomycin distribution and clearance due to pathophysiological changes and concomitant therapies compromise the attainment of predefined exposure targets (2). For instance, fluid resuscitation and total parenteral nutrition expand the extracellular fluid content and dilute serum and extracellular vancomycin concentrations $(3,4)$. On the other hand, renal function is often impaired in the critically ill causing accumulation of the infused drug (2). To reduce the variability of vancomycin exposure and increase target attainment rates, switching the mode of administration from intermittent $(\operatorname{lnl})$ to continuous infusion (Col) has been proposed (5). In this study we primarily investigated the within-population variability in vancomycin exposure of Inl and Col in critically ill patients. Secondly, vancomycin serum concentrations and AUC correlations and target attainment rates were compared between Inl and Col groups. 


\section{Methods}

A retrospective cohort study was conducted among critically ill patients admitted to a level 2 intensive care unit between April 2007 and November 2013. Adult patients (18 years or older) treated with intravenous vancomycin for various indications including sepsis, pneumonia and endocarditis were eligible for inclusion. Patients were excluded if treated with any form of renal replacement therapy (RRT) or if vancomycin was prescribed in the three days prior to initiation of therapy. A waiver for consent was provided by the Medical Ethical Committee. Vancomycin was administered through intravenous infusion and dosing was guided by therapeutic drug monitoring (TDM). Before 2010, vancomycin was administered as an intermittent infusion. Vancomycin was started as a $15 \mathrm{mg} / \mathrm{kg}$ infusion every 12 hours. First trough (Conc1) and peak (two hours after the end of infusion) serum samples were obtained 24 hours after initiation of therapy and a second set of peak and trough (Conc2) samples were obtained at day 2 or 3 of therapy. The predefined target trough serum concentration for Inl was 8-12 mg/L. In 2010, the mode of administration switched from Inl to Col. A standard loading dose of $1000 \mathrm{mg}$ infused over 2 hours directly followed by a glomerular filtration rate (GFR) based maintenance dose according to a prospectively validated nomogram (6)). First and second serum plateau samples were drawn approximately 20-36 (Conc1) and 36-72 (Conc2) hours after initiation of therapy, respectively. The target plateau concentration for Col was $15-20 \mathrm{mg} / \mathrm{L}$. Vancomycin serum samples were assayed using a fluorescence polarization immunoassay (TDx, Abbott, Hoofddorp, the Netherlands). AUC0-24h and AUC24-48h were estimated in all individuals using a pharmacokinetic population model and pharmacokinetic software (Appendix 1). Variability in serum concentrations and AUC's was represented by the relative standard deviations (RSD's) as defined by the formula: $R S D=100$ * mean/SD, in which SD represents the standard deviation. Subsequently, SD's were compared between Inl and Col groups. Secondly, coefficients of determination ( $\mathrm{r} 2$ ) between serum concentrations and AUC's were obtained by univariate linear regression analyses. Finally, target attainment rates of Inl and Col were determined for serum concentrations and AUC24h values prior to (Conc1 and AUC0-24h) and after (Conc 2 and AUC2448h) TDM-based dose adjustment. A 'therapeutic' AUC was defined as an AUC24h $\geq 350$ mg.h.L-1 (7, 8). Clinical outcomes could not be 
assessed due to the small number of patients.

\section{Results}

A total of 113 patients were selected. Five patients were excluded for RRT and 25 for recent prior treatment with vancomycin. Pharmacokinetic data were not available in five patients. Dosing protocol violations excluded seven patients. The remaining 71 patients were included. Baseline characteristics of included patients are shown in Table 1. No significant differences were seen between the two treatment groups. In all occasions the variability in serum concentrations and in AUC's was higher in patients treated with vancomycin Inl compared to Col (Table 2). Linear regression analysis showed that coefficients of determination ( $r 2$ ) were higher and 95\%-confidence intervals were nearly twice as large in the Inl versus the Col treated population on both sampling occasions (Figure 1). Target attainment rates were significantly higher in the Col versus the Inl group for both AUC0-24h (48.0 versus 18.5, $\mathrm{p}<0.001)$ and Conc1 (61.4 vs 14.8, $\mathrm{p}<0.001)$. 
Table 1 Patient characteristics

\begin{tabular}{|c|c|c|c|}
\hline & InI (n=27) & Col $(n=44)$ & p-value \\
\hline Male gender, \% (n) & 66.7 (18) & $65.9(29)$ & $0.948 a$ \\
\hline Age (y) (meanıSD) & $67.8 \pm 12.2$ & $66.6 \pm 12.5$ & $0.702 b$ \\
\hline Weight (kg) (mean \pm SD) & $79.8 \pm 19.4$ & $78.4 \pm 18.5$ & $0.765 b$ \\
\hline Height (cm) (mean $\pm S D)$ & $174 \pm 9.0$ & $173.9 \pm 8.8$ & $0.858 b$ \\
\hline $\begin{array}{l}\text { SAPS II score } \\
\text { (mean, range) }\end{array}$ & $41(12-79)$ & $42(13-91)$ & $0.790 c$ \\
\hline $\begin{array}{l}\text { Baseline creatinine } \\
\text { clearance }(\mathrm{ml} / \mathrm{min}) \\
(\text { mean } \pm \mathrm{SD})\end{array}$ & $93 \pm 52.91$ & $78 \pm 37.0$ & $0.11 b$ \\
\hline $\begin{array}{l}\text { Baseline serum creatinine } \\
(\mu \mathrm{mol} / \mathrm{L})(\mathrm{mean} \pm \mathrm{SD})\end{array}$ & $86 \pm 38$ & $99 \pm 39$ & $0.13 b$ \\
\hline $\begin{array}{l}\text { Baseline serum urea } \\
(\mathrm{mmol} / \mathrm{L})(\mathrm{mean} \pm \mathrm{SD})\end{array}$ & $16.3 \pm 6.3$ & $14.4 \pm 7.3$ & $0.28 b$ \\
\hline
\end{tabular}

P-values were determined by; $a$, Pearson Chi-Square test; $b$, Independent Samples Test; and c Mann-Whitney U Test. Abbreviations are Col, continuous infusion; Inl, intermittent infusion; SAPS, simplified acute physiology score; and SD, standard deviation. 
Table 2. Exposure variability and target attainment rates

\begin{tabular}{|c|c|c|c|c|c|c|c|c|c|}
\hline \multirow[b]{3}{*}{$\begin{array}{l}\text { Conc } 1 \\
\text { (mg/L) }\end{array}$} & \multicolumn{4}{|c|}{ Intermittent infusion } & \multicolumn{5}{|c|}{ Continuous infusion } \\
\hline & Mean & SD & $\begin{array}{l}\text { RSD } \\
(\%)\end{array}$ & $\begin{array}{c}\text { Target } \\
\text { attainment } \\
(\%)\end{array}$ & Mean & SD & $\begin{array}{l}\text { RSD } \\
(\%)\end{array}$ & $\begin{array}{c}\text { Target } \\
\text { attainment } \\
(\%)\end{array}$ & $\begin{array}{l}\text { p-value (target } \\
\text { attainment) }\end{array}$ \\
\hline & 9.3 & 4.6 & 49.2 & 18.5 & 16.4 & 4.7 & 28.7 & 48.0 & $<0.001$ \\
\hline $\begin{array}{l}\text { Conc } 2 \\
\text { (mg/L) }\end{array}$ & 15.2 & 6.9 & 45.1 & 23.8 & 21.0 & 5.2 & 24.5 & 33.3 & 0.471 \\
\hline $\begin{array}{l}\mathrm{AUC}_{0-24 h} \\
\left(\mathrm{mg} \cdot \mathrm{h} \cdot \mathrm{L}^{-1}\right)\end{array}$ & 289 & 88 & 30.4 & 14.8 & 365 & 91 & 24.9 & 61.4 & $<0.001$ \\
\hline $\begin{array}{l}\mathrm{AUC}_{24-48 \mathrm{~h}}, \\
\left(\mathrm{mg} \cdot \mathrm{h} \cdot \mathrm{L}^{-1}\right)\end{array}$ & 475 & 161 & 33.3 & 76.2 & 476 & 108 & 22.7 & 88.9 & 0.242 \\
\hline
\end{tabular}

Serum concentrations 1 and 2 were sampled shortly prior to the administration of the third and fifth to seventh administration in the intermittently infused patients. First and second serum samples in continuously infused patients were drawn approximately 20-36 (Conc1) and 36-72 (Conc2) hours after initiation of therapy, respectively. AUC's were estimated using a PK model and Bayesian based PK software. The target plateau concentration for continuous infusion was $15-20 \mathrm{mg} / \mathrm{L}$. Predefined target trough serum concentration for intermittent infusion was 8-12 mg/L. Abbreviations are AUC, area under the concentration-time curve; Conc 1, serum concentration 1; Conc 2, serum concentration 2; Col, continuous infusion; Inl, intermittent infusion; RSD, relative standard deviation; and SD, standard deviation. 


\section{Figure 1. Serum concentration and AUC Correlations}

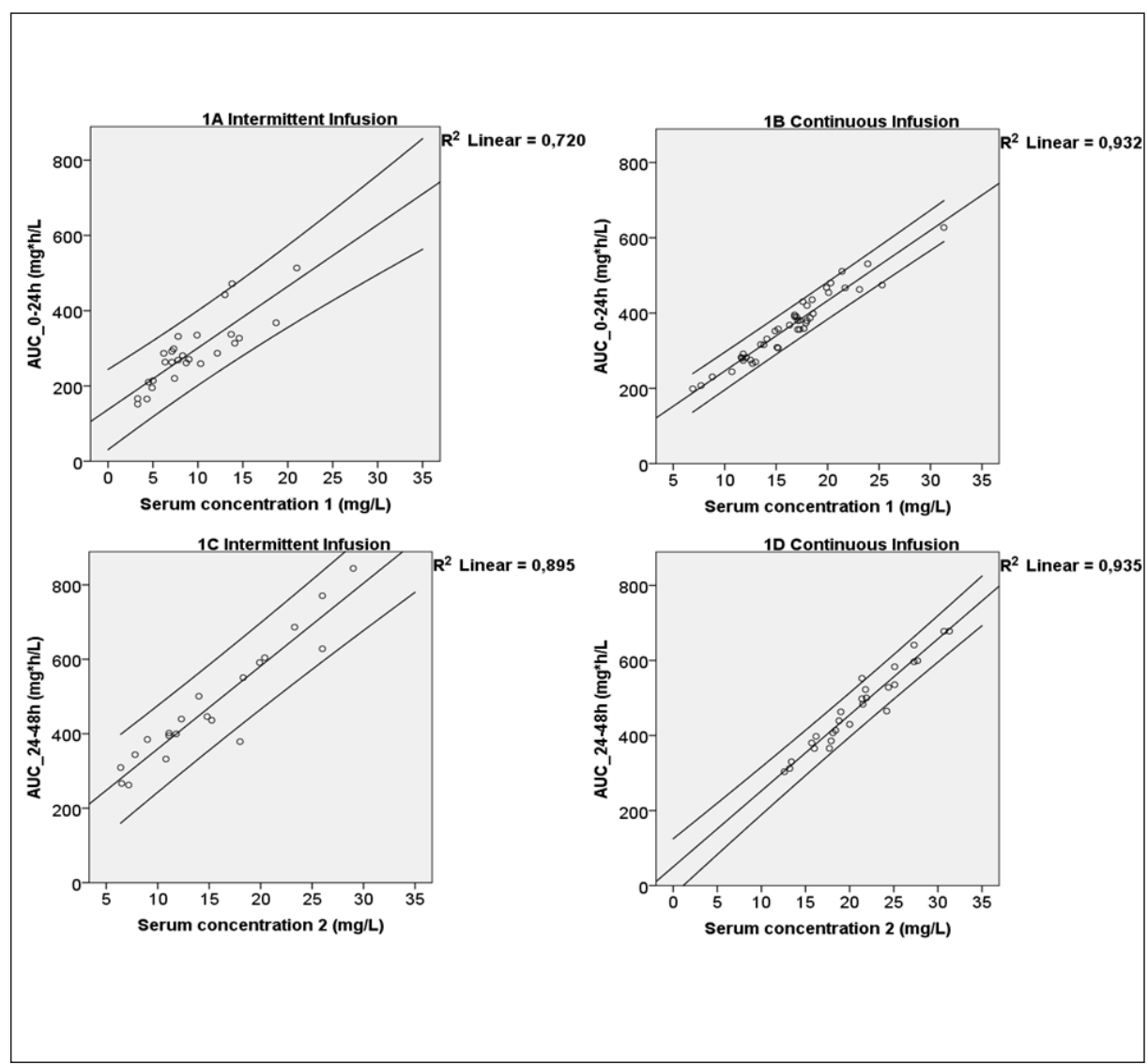

Serum concentration and AUC Correlations in intermittently infused patients are shown in figures $1 \mathrm{~A}$ and $1 \mathrm{C}$. Correlations in the continuously infused group are depicted in $1 \mathrm{~B}$ and 1D. In intermittently dosed patients serum concentrations 1 and 2 were sampled shortly prior to the administration of the third and fifth to seventh administration, respectively. First and second serum samples in continuously infused patients were drawn approximately 20-36 and 36-72 hours after initiation of therapy, respectively. AUC's were estimated using a PK model and Bayesian based PK software. The outer lines represent $95 \%$-confidence intervals. All correlations were significant at a $\mathrm{p}$-level of $<0.01$. 


\section{Discussion}

To the best of our knowledge, this is the first study to directly compare exposure variability of vancomycin Inl and Col in critically ill patients. The results show lower variability and higher target attainment rates of serum concentrations and AUC's in patients treated with vancomycin Col compared to those treated with Inl. Importantly, the correlation between serum concentrations and AUC's was better in the Col compared to the Inl group. Consequently, a serum 'plateau' concentration in Col will be a better predictor of patients' exposure in terms of AUC compared to a trough concentration in InI. Since recent guidelines from the Infectious Disease Society of America (IDSA) recommend trough serum concentrations of $15-20 \mathrm{mg} / \mathrm{L}$ to attain a minimal AUC24h of 400 mg.h.L-1 $(8,10)$, we were also interested in the relation between concentrations and AUC's. Although it was not one of the primary aims of our study, we found that trough serum concentrations between 12-16 $\mathrm{mg} / \mathrm{L}$ resulted in AUC24h values over $400 \mathrm{mg} . \mathrm{h} . \mathrm{L}-1$ in vancomycin Inl (Figure 1). These findings are supported by recent data published by Neely and coworkers (11). Ultimately, dose adjustments in vancomycin Inl based on current trough concentration recommendations render needlessly high exposure in critically ill patients infected by (suspected) pathogens with a MIC $\leq 1 \mathrm{mg} / \mathrm{L}$ with an increased toxicity risk as a resultant. The study is primarily limited by its open and retrospective design, although patient baseline characteristics were well balanced. Secondly, the poor performance of the Inl regimen with regard to AUC target attainment may result from the fact that this regimen was not designed to target AUC's $>350$ mg.h.L-1. Our findings can be explained by the fact that variability in 'plateau' concentrations during vancomycin Col is only dependent of variability in clearance, whilst trough concentration variability reflects the variation in clearance and volume of distribution. Moreover, 'trough sample timing' will significantly affect the variability in actually measured concentrations during Inl. 'Plateau' concentrations in patients on vancomycin Col, on the other hand, are relatively independent of the sampling time under steady state conditions. (12). In conclusion, we have demonstrated that continuous infusion leads to more robust vancomycin exposure and can beneficially affect AUC target attainment in the critically ill. Whether this advantage leads to improved clinical outcomes warrants further investigations. 


\section{Funding}

This work was not externally supported. All work was funded from the departments' research budgets.

\section{Transparency declarations}

None of the authors has conflicts of interest to declare. 


\section{References}

1. Moise-Broder PA, Forrest A, Birmingham MC, Schentag JJ. Pharmacodynamics of vancomycin and other antimicrobials in patients with Staphylococcus aureus lower respiratory tract infections. Clin Pharmacokinet 2004;43(13):925-42.

2. Roberts JA, Lipman J. Pharmacokinetic issues for antibiotics in the critically ill patient. Crit Care Med 2009;37(3):840-51; quiz 859.

3. Pea F, Viale P, Furlanut M. Antimicrobial therapy in critically ill patients: a review of pathophysiological conditions responsible for altered disposition and pharmacokinetic variability. Clin Pharmacokinet 2005;44(10):100934.

4. Scaglione F, Paraboni L. Pharmacokinetics/pharmacodynamics of antibacterials in the Intensive Care Unit: setting appropriate dosing regimens. Int J Antimicrob Agents 2008;32(4):294-301.

5. Smuszkiewicz P, Szalek E, Tomczak H, Grzeskowiak E. Continuous infusion of antibiotics in critically ill patients. Curr Clin Pharmacol;8(1):13-24. 6. van Maarseveen EM, Bouma AW, Touw DJ, Neef C, van Zanten AR. Unpublished data: Validated eGFR based Dosing Instrument for Continuous Infusion of Vancomycin in Hospitalized Patients. Eur J Clin Pharm, sept 2014 (epub ahead of print)

7. Rybak M, Lomaestro B, Rotschafer JC, Moellering R, Jr., Craig W, Billeter $\mathrm{M}$, et al. Therapeutic monitoring of vancomycin in adult patients: a consensus review of the American Society of Health-System Pharmacists, the Infectious Diseases Society of America, and the Society of Infectious Diseases Pharmacists. American Journal of Health-System Pharmacy: AJHP: Official Journal of the American Society of Health-System Pharmacists 2009;66(1):82. 8. Rybak MJ, Lomaestro BM, Rotschafer JC, Moellering RC, Craig WA, Billeter M, et al. Vancomycin therapeutic guidelines: a summary of consensus recommendations from the infectious diseases Society of America, the American Society of Health-System Pharmacists, and the Society of Infectious Diseases Pharmacists. Clin Infect Dis 2009;49(3):325-7.

9. Bellomo R, Ronco C, Kellum JA, Mehta RL, Palevsky P, Acute Dialysis Quality Initiative w. Acute renal failure - definition, outcome measures, animal models, fluid therapy and information technology needs: the Second International Consensus Conference of the Acute Dialysis Quality Initiative (ADQI) Group. Critical Care (London, England) 2004;8(4):R204.

10. Rybak MJ, Lomaestro BM, Rotschafer JC, Moellering RC, Jr., Craig WA, Billeter M, et al. Therapeutic monitoring of vancomycin in adults summary of consensus recommendations from the American Society of Health-System Pharmacists, the Infectious Diseases Society of America, and the Society of Infectious Diseases Pharmacists. Pharmacotherapy 2009;29(11):1275-9. 11. Neely MN, Youn G, Jones B, Jelliffe RW, Drusano GL, Rodvold KA, 
et al. Are vancomycin trough concentrations adequate for optimal dosing? Antimicrob Agents Chemother;58(1):309-16.

12. van Maarseveen EM, Gipmans SGH, Vasbinder EC, Petjak M, van Zanten AR. Unpublished data: Variability of exposure in critically ill patients receiving intermittent or continuous infusion of vancomycin - are we on target? In.

13. Proost JH, Meijer DK. MW/Pharm, an integrated software package for drug dosage regimen calculation and therapeutic drug monitoring. Comput Biol Med 1992;22(3):155-63.

14. Pullen J, de Rozario L, Stolk LM, Degraeuwe PL, van Tiel FH, Zimmermann LJ. Population pharmacokinetics and dosing of flucloxacillin in preterm and term neonates. Ther Drug Monit 2006;28(3):351-8. 
Appendix 1. Brief description of the design and validation of the pharmacokinetic model

Manuscript title: 'Exposure variability and target attainment of vancomycin intermittent and continuous infusion in critically ill patients ready, aim, target locked?'

\# corresponding author:

Erik M. van Maarseveen

University Medical Center Utrecht

Department of Clinical Pharmacy, Room HP D00.218

Heidelberglaan 100

P.O.Box 85500, 3508 GA Utrecht The Netherlands

Telephone:

$+31-88-7557218$

Fax: $\quad+31-88-7556756$

Email: $\quad$ E.M.vanmaarseveen@UMCUtrecht.nl

\section{Model design}

Data were collected from patient clinical records and the electronic prescription database at the $\mathrm{GHZ}$. A pharmacokinetic population model was designed using the KinPop module of MWMPharm (version 3.80, MedilWare, Zuidhorn, the Netherlands) for each of the groups using the trough and peak serum concentration obtained from patients that received vancomycin through intermittent infusion. MWIPharm uses an iterative two-stage Bayesian approach to estimate the mean and coefficient of variation of relevant pharmacokinetic parameters. Population models were designed and validated according to previously published methodology (14). The following polynomial formula was used to estimate the SD (standard deviation) of the analytical assay error: SD $=0.323+0.0265{ }^{*} \mathrm{C}+0.0002{ }^{*} \mathrm{C} 2$. A two-compartment model was designed. The elimination rate constant was defined by the formulae: $\mathrm{Kel}\urcorner=\mathrm{Kelm}+\mathrm{Kelr}$ and Kelr $=$ Kslope ${ }^{*} \mathrm{Clcr}$, in which Kelm, Kelr and Kslope represent the metabolic elimination constant, renal elimination constant and slope constant, respectively. The free fraction of vancomycin was assumed to be constant at $0.5 \pm 0.1$ in the model. 
The model was evaluated by bootstrap analysis ( $n=1000$ replicates), an analysis of individual predicted versus observed concentrations, an analysis of the residual error versus the observed concentrations and model fit was checked visually for all individual patients. A pharmacokinetic model was established. Bootstrap analysis resulted similar parameters estimates (Table A1). Predicted versus observed serum concentrations plot showed good correlation $(r 20.88, p<0.01)$ and weighted residuals were randomly distributed over the concentration range (Figure A1). Visual check of all individual concentration-versus-time-curves showed good individual fits.

\begin{tabular}{lllll} 
& \multicolumn{2}{c}{ PK model parameters } & \multicolumn{2}{l}{ Bootstrap estimates } \\
& Estimate & CV (\%) & Mean & RSE (\%) \\
\hline Kelm (h-1) & 0.017 & 43 & 0.019 & 56 \\
$\begin{array}{l}\text { Kslope } \\
\text { (h-1/(ml min-1)) }\end{array}$ & 0.0016 & 31.4 & 0.0015 & 17.1 \\
V1 (L/kg) & 0.47 & 18.4 & 0.51 & 14.6 \\
K12 (h-1) & 2.9 & 51.0 & 3.2 & 48.0 \\
K21 (h-1) & 1.1 & 106.5 & 0.9 & 40.4 \\
\hline
\end{tabular}

Table A1. Pharmacokinetic population estimates and bootstrap analysis results of Inl model in critically ill patients. Abbreviations: ICU, intensive care unit; Inl, intermittent infusion; Kelm, metabolic elimination rate constant; Kslope, renal elimination rate constant per $\mathrm{ml} / \mathrm{min}$ clearance; $\mathrm{PK}$, pharmacokinetic; CV, coefficient of variation; RSE, relative standard error; V1, volume of distribution in the central compartment. 


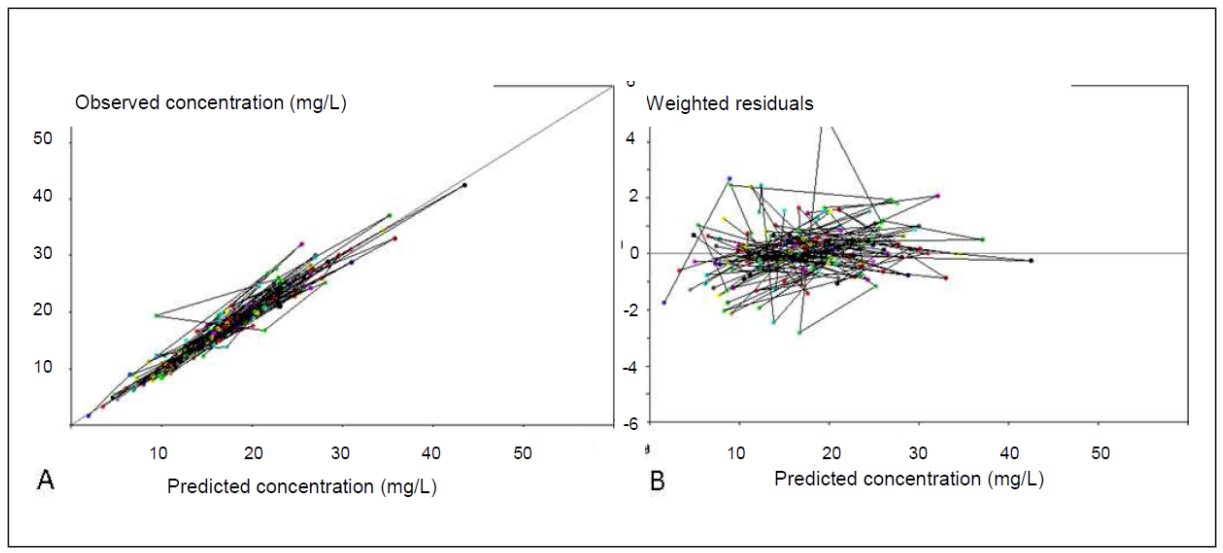

Figure A1. Plot A shows the individual predicted versus observed concentrations of the pharmacokinetic population model. In plot B the weighted residuals over the measured concentrations are depicted. 


\title{
Chapter 3.3
}

Design and Prospective Validation of a Dosing Instrument for Continuous Infusion of Vancomycin: A Within-population Approach

\author{
ERIK M. VAN MAARSEVEEN \\ ANNEMIEN BOUMA \\ DANIEL J. TOUW \\ CEES NEEF \\ ARTHUR R.H. VAN ZANTEN
}

Eur J Clin Pharmacol. 2014 Aug 30. [Epub ahead of print] 


\section{Abstract}

The clinical application of continuous infusion (Col) of vancomycin is rapidly gaining interest. Since no international guidelines on initial dosing of vancomycin Col exist, there is an urgent need for methods to facilitate the switch from intermittent to continuous vancomycin dosing algorithms in clinically infected populations. Therefore, the aim of this study was to describe the design and validation of an a priori dosing schedule for Col of vancomycin in clinical practice. A dosing table for Col of vancomycin based on estimated glomerular filtration rate (eGFR) was developed by simulation of continuous infusion of vancomycin using pharmacokinetic (PK) software and a PK population model designed from historical within-population data in intermittently dosed patients. The target range for steady state concentration (Css) at 24 hours was set at $15-20 \mathrm{mg} / \mathrm{L}$ corresponding with an area-under-thecurve (AUC) of at least $350 \mathrm{mg} \cdot \mathrm{kg} \cdot \mathrm{L}-1$. The performance of the dosing schedule was primarily assessed by describing the percentages of patients attaining the predefined target. An eGFR-derived dosing schedule for Col of vancomycin was established and implemented in clinical practice. Prospective assessment in 35 general ward and 45 intensive care unit patients showed that AUC target was attained in $80 \%$ and $72 \%$ of patients and Css target was reached in $63 \%$ and $69 \%$, respectively. An easy method to design and validate an eGFR-derived dosing algorithm for the continuous infusion of vancomycin to switch from intermittent to continuous dosing of vancomycin is made available.

\section{Introduction}

Vancomycin is a glycopeptide antibiotic used in (suspected or proven) bacteraemia, peritonitis or osteomyelitis with gram-positive micro-organisms. Antimicrobial killing of vancomycin is time and/or area-under-the-curve dependent and requires therapeutic concentrations of vancomycin at the site of infection $(1,2)$. Although resistance against vancomycin and other glycopeptides is emerging worldwide among methicillin-resistant Staphylococcus aureus and vancomycin-intermediate S. aureus (VISA) strains (3), vancomycin still is the antibiotic of choice for the therapy of methicillin-resistant $S$. aureus (MRSA) infections according to recent guidelines from the Infectious Diseases Soci- 
ety of America (IDSA) (4). To preserve the current therapeutic armamentarium for Gram-positive infections, it is of paramount importance that guidelines for vancomycin dosing are based on patient outcomes and on in vitro and in vivo pharmacokinetic/ pharmacodynamic (PK/ PD) data to attain maximum efficacy with minimal toxicity and prevent the advent of resistance. Albeit there is in vitro evidence showing that the time above the minimal inhibitory concentration (MIC) and AUC/ MIC-values are the most important parameters associated with efficacy, most human studies failed to detect a clear PK/PD relationship (2). To date, only Moise-Broder et al. showed that for intermittent infusion of vancomcyin an 'in vivo' AUC/MIC-ratio of $<350$ was associated with a Ihigher cure rate $(P=0.005)$ in patients with MRSA pneumonia (1). Classically, vancomycin is administered as an intermittent infusion of $15-20 \mathrm{mg} / \mathrm{kg}$ every 12 hours. (5). Unfortunately, ascertaining AUC/ MIC values $>350 \mathrm{mg} \cdot \mathrm{kg} \cdot \mathrm{L}-1$ and concentrations above MIC shortly after initiation of therapy can be difficult with conventional intermittent dosing in combination with trough level monitoring. To optimize the pharmacodynamics of vancomycin higher intermittent daily dosages have been suggested (6-8). Nevertheless, even with higher intermittent dosing attaining therapeutic exposure remains a challenge in patients with rapid clearance of vancomycin. Hence, it seems rational to explore alternative dosing strategies of vancomycin, e.g. continuous infusion (Col). Recent data suggest that administration of vancomycin for the treatment of Gram-positive infections by Col is associated with a significantly lower risk of nephrotoxicity compared to intermittent infusion, while efficacy parameters were comparable (9-11). In addition, continuous infusion of vancomycin is feasible due to demonstrated stability at temperatures up to $37^{\circ} \mathrm{C}$ for 72 hours (12) and might also be preferred due to a reduction in drug preparation time, staff workload, costs and hospital length of stay $(13,14)$.

Gradually, more data on the therapeutic range of Col are generated. Based on the pragmatic approach of achieving AUC's equal to intermittent dosing a minimum plateau level of $15 \mathrm{mg} / \mathrm{L}$ corresponding with an AUC24h of $>350 \mathrm{mg} \cdot \mathrm{kg} \cdot \mathrm{L}-1$ most studies used $15-25 \mathrm{mg} / \mathrm{L}$ as a target steady state concentration (Css) (15-19). These studies showed microbiological and clinical efficacy rates in the Col study arm that were equivalent or better than the study arm receiving vancomycin via intermittent infusion. Only scarce data on the correlation between plateau levels and rates of nephrotoxicity exist. Studies performed by Spapen et al. and Ingham et al. showed plateau levels exceeding $28-30 \mathrm{mg} / \mathrm{L}$ 
were significantly associated with nephrotoxicity both in critically ill and outpatients populations $(20,21)$. For comparison, serum trough concentrations over $15-20 \mathrm{mg} / \mathrm{L}$ during intermittent dosing of vancomycin have been associated with an increased risk of nephrotoxicity $(22,23)$. As the clinical application of Col of vancomycin is rapidly gaining popularity and no international guidelines exist, there is an urgent need for methods to design dosing algorithms for continuous vancomycin dosing algorithms aiming for predefined AUC/MIC exposure targets in clinically infected populations. Therefore, the aim of this study was to describe the design and validation of a practical and easy to apply dosing schedule for vancomycin $\mathrm{Col}$ in clinical practice.

\section{Methods}

\section{Study design}

Prior to prospective validation a dosing table for Col of vancomycin was developed by simulation of continuous infusion of vancomycin using the PK software package MWPharm (version 3.60, MedilWare, Zuidhorn, The Netherlands) (24) and a pharmacokinetic (PK) population model based on historical within-population data of patients on intermittently dosed vancomycin. The PK model was validated using a previously described methodology (25) (Appendix 1). The parameters of the PK model were: V1, $0.29 \mathrm{~L} / \mathrm{kg} ; \mathrm{k} 12,0.92 / \mathrm{h}$ and $\mathrm{k} 21,0.46 / \mathrm{h}$; Kslope, $0.00372 / \mathrm{h} /(\mathrm{ml} / \mathrm{min}$ ) and Kelm, $0.018 / \mathrm{h}$. The elimination rate constant was defined by the formulae: Kel $=$ Kelm + Kelr and Kelr $=$ Kslope * ${ }^{*}$ _cr, in which Kelm, Kelr and $\mathrm{Cl}$ cr reflect the metabolic rate constant, renal elimination slope constant and creatinine clearance, respectively. The free fraction of vancomycin was assumed to be constant in the model. Vancomycin clearance is the key parameter for prediction of Css. The volume of distribution (Vd) is required to accurately determine the total serum AUC of the first 24 hours after start of infusion in individual patients. Patients with an estimated glomerular filtration rate (eGFR) based on the modification of diet in renal disease (MDRD) formula ranging from 10 to $150 \mathrm{~mL} / \mathrm{min} / 1.73 \mathrm{~m} 2$ were simulated with intervals of $5 \mathrm{~mL} / \mathrm{min}$ (26). Simulated patients' weights ranged from 50 $100 \mathrm{~kg}$. A target range for Css at 24 hours of $15-20 \mathrm{mg} / \mathrm{L}$ corresponding with an AUC/MIC ratio of $>350$, was set to determine a fixed loading dose and individualized maintenance dosages within a range of eGFR based on MDRD and simulated weights. 
For the prospective validation adult patients admitted to a 400 bed teaching hospital starting with Col of vancomycin were eligible for inclusion. A waiver was provided by the medical ethical committee. Patients were excluded if they were on renal replacement therapy (RRT) or if the administered loading or maintenance dose deviated from the dosing table. Vancomycin clearances and AUC's of the first 24 hours after initiation of therapy were estimated in all patients with maximum a posteriori Bayesian estimation (MWMPharm 3.60). A two compartment open PK model was used. Accuracy and precision of the dosing table were assessed by calculation of the percentages of patients reaching a Css of $15-20 \mathrm{mg} / \mathrm{L}$ or an AUC $>350 \mathrm{mg} \cdot \mathrm{kg} \cdot \mathrm{L}-1$. Finally, agreement between predicted and observed Css values was analyzed by testing correlation and by assessment of bias, means and $95 \%$ confidence intervals $(95 \% \mathrm{Cl})$ of observed versus predicted values.

\section{Vancomycin and creatinine assays}

Serum concentrations of vancomycin were drawn 18-28 hours after initiation of infusion, when steady state was assumed in the majority of patients. A second vancomycin serum concentration was drawn 72 hours after initiation of therapy. Samples were analyzed using a fluorescence polarization immunoassay (TDx, Abbott, Hoofddorp, The Netherlands). The lower limit of quantification and intraday and between-day coefficients of variation for vancomycin were $0.7 \mathrm{mg} / \mathrm{L}$ and 0.8 and $1.2 \%$, respectively. The linearity ( $r 2$ ) of the assay was 0.998 . Baseline serum creatinine was analyzed using a colorimetric method (DxC, Beckman Coulter Inc., Brea, CA, USA). GFR was estimated using the modified diet in renal disease (MDRD) formula and serum creatinine values drawn within 24 or 48 hours before the initiation of vancomycin in the intensive care unit and general ward population, respectively. 


\section{Statistical analysis}

As the PK parameters of the intensive care unit and general ward populations were expected to differ, patients were divided into 2 groups. Data were tested for normal distribution and variance homogeneity with Kolmogorov-Smirnoff and Levene tests. Means and standard deviations of continuous variables are presented in normal distributions. Otherwise, medians and ranges were reported. A Student's t-test was used to determine statistical differences among normally distributed groups. If data were not normally distributed, a Mann-Whitney-U test was performed. A Chi-square test was used to compare dichotomous variables between groups. Predicted vs. observed Css levels at 18-28 hours after initiation of therapy were compared using Pearson correlation. Univariate and multivariate analyses of patient characteristics were performed to identify determinants of underexposure (AUC $<350 \mathrm{mg} \cdot \mathrm{k}$ $\mathrm{g} \cdot \mathrm{L}-1$ and $\mathrm{Css}$ level $<15 \mathrm{mg} / \mathrm{L}$ ) by binary logistic regression. A stepwise backward elimination procedure was carried out, retaining covariates in the model only when they were statistically significant. To account for multiple testing, only those covariates were tested that were shown to affect the risk of underexposure in univariate analyses and a stringent $P$ value $(<0.005)$ was selected. To evaluate the effect of renal function on exposure, patients were divided into 4 groups based on data distribution of MDRD-derived eGFR: MDRD $<40 \mathrm{ml} / \mathrm{min} / 1.73 \mathrm{~m} 2(\mathrm{n}=17)$, $40 \leq \mathrm{MDRD}<60 \mathrm{ml} / \mathrm{min} / 1.73 \mathrm{~m} 2(\mathrm{n}=15), 60 \leq \mathrm{MDRD}<90 \mathrm{ml} / \mathrm{min} / 1.73 \mathrm{~m} 2$ $(n=19)$, and MDRD $>90 \mathrm{ml} / \mathrm{min} / 1.73 \mathrm{~m} 2(n=29)$. To test the effect of body weight on achieving therapeutic exposure were divided into quartiles based on data distribution. Statistical significance was defined by $P$-values $<0.05$. All tests were performed in SPSS Statistics version 20 (SPSS Inc., Chicago, IL, USA). 


\section{Results}

A dosing table for Col was established (Table 1).

Table 1. Dosing table for vancomycin continuous infusion

\begin{tabular}{|ll|}
\hline & \\
\hline eGFR $^{\mathrm{a}}\left(\mathrm{ml} / \mathrm{min} / 1.73 \mathrm{~m}^{2}\right)$ & Dose $^{\mathrm{b,c}}(\mathrm{mg} / 24 \mathrm{~h})$ \\
$30-39$ & 500 \\
$40-49$ & 750 \\
$50-59$ & 1000 \\
$60-79$ & 1250 \\
$80-89$ & 1500 \\
$>90$ & 1750 \\
\hline
\end{tabular}

a glomerular filtration rate estimated with MDRD formula

b target Css: $15-20 \mathrm{mg} / \mathrm{L}$

c preceded by a loading dose of $1000 \mathrm{mg}$ administered over 2 hours

In total 103 patients were treated with Col of vancomycin between January 2009 and August 2012. Ten patients were excluded for dosing protocol violations and 13 patients were excluded of reasons of RRT. Patient characteristics are shown in Table 2. 
Table 2. Baseline characteristics

\begin{tabular}{|c|c|c|c|}
\hline & \multicolumn{2}{|c|}{ Population } & \multirow[b]{2}{*}{ p-value } \\
\hline & General ward & Intensive care unit & \\
\hline No. patients & 35 & 45 & \\
\hline $\begin{array}{c}\text { Wards (\%) } \\
\text { Surgical } \\
\text { Medical }\end{array}$ & $\begin{array}{l}47 \\
53\end{array}$ & n.a. & \\
\hline SAPS-II score & n.a. & $41(12-79)$ & \\
\hline age $(y)$ & $71(12.4)$ & $70(12.3)$ & $0.95^{\mathrm{a}}$ \\
\hline male gender (\%) & 54 & 58 & $0.36^{\mathrm{b}}$ \\
\hline weight (mg) & $75.9(14.3)$ & $76.6(15.8)$ & $0.83^{\mathrm{a}}$ \\
\hline $\operatorname{MDRD}\left(\mathrm{mL} / \mathrm{min} / 1.73 \mathrm{~m}^{2}\right)$ & $65(13->90)$ & $72(24->90)$ & $0.60^{\mathrm{c}}$ \\
\hline
\end{tabular}

Baseline characteristics of included patients are shown. Differences in categorical variables were expressed as numbers and percentages, and continuous variables as means and standard deviations if normally distributed or as medians and range between parentheses if not normally distributed. Abbreviations are: eGFR, glomerular filtration rate estimated with MDRD formula; n.a., not applicable.

a Student's t-test,

b Chi-squared test,

c Mann-Whitney-U test

All eligible patients who received vancomycin demonstrated infections caused by documented or presumed vancomycin sensitive pathogens. Included vancomycin samples were drawn 18-28 hours after initiation of therapy. The dosing table produced good accuracy and precision of observed Css levels in intensive care unit and general ward patients (Figure 1 and Table 3).

No significant differences were found in mean Css levels at 24 hours, AUC's and clearance of vancomycin between the intensive care unit and general ward populations (Table 3). 
Figure 1: Frequency distribution of vancomycin serum concentrations and total serum area-under-the-curves

Figure 1A

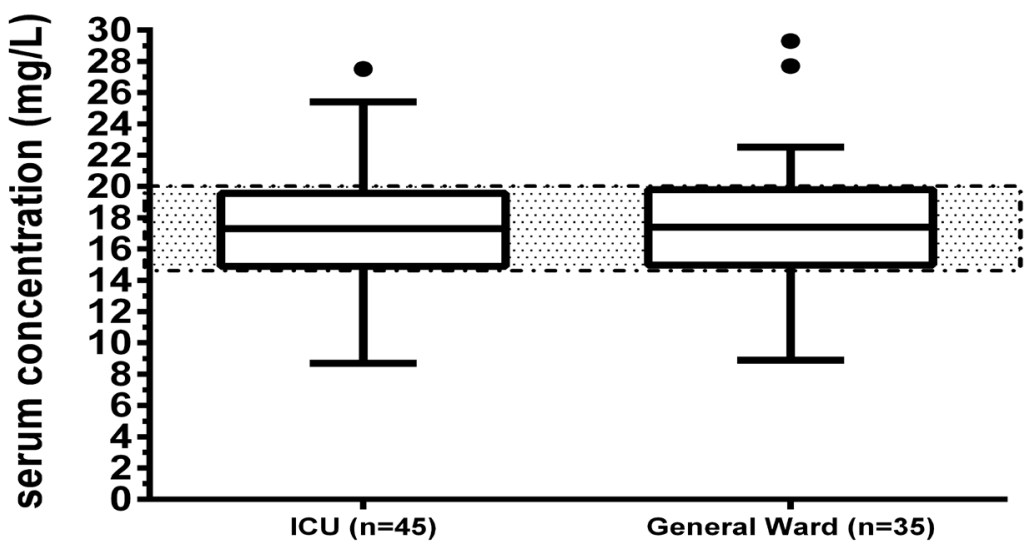

Figure 1B

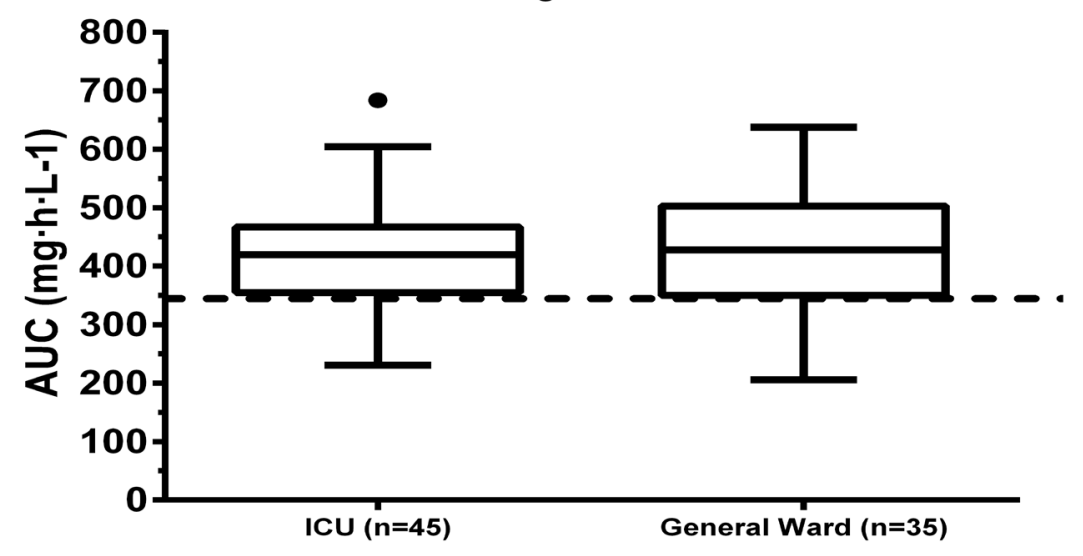

Box-and-whisker plots of vancomycin total serum concentrations at 24 hours after initiation of therapy $(1 \mathrm{~A})$ and total serum area-under-the-curves (AUC's) on day 1 of therapy $(1 \mathrm{~B})$ are displayed for the general ward and intensive care unit populations. The line in the middle of each box represents the median; the box extends from the 25th to 75th percentile (interquartile range); and the lines emerging from the box extend to three quarters of the interquartile range, rolled back to where data are present. The shaded area in Figure 1A indicates the therapeutic target concentration range $(15-20 \mathrm{mg} / \mathrm{L})$. The interrupted line in Figure 1B indicates the minimal AUC target of $350 \mathrm{mg} \cdot \mathrm{h} \cdot \mathrm{L}-1$. 
Table 3 Dosing and pharmacokinetic parameters

\begin{tabular}{|c|c|c|c|}
\hline & \multicolumn{2}{|c|}{ Population } & \multirow[b]{2}{*}{ p Value } \\
\hline & General ward & Intensive care unit & \\
\hline Initial Dose (mg) & $1500(500-2000)$ & $1500(500-2000)$ & $0.35 c$ \\
\hline Sampling time (h) & $22.5(4.2)$ & $25.8(3.1)$ & $0.76 a$ \\
\hline $\mathrm{Css}(\mathrm{mg} / \mathrm{mL})$ & $17.4(5.0)$ & $17.4(5.3)$ & $0.94 a$ \\
\hline Clvanco (L/h) & $3.6(1.7)$ & $3.6(0.9)$ & $0.61 a$ \\
\hline \multicolumn{4}{|l|}{ Total serum } \\
\hline AUC0-24h (mg*h/L) & $427(107)$ & $415(112)$ & $0.63 a$ \\
\hline Dose adjustment (\%) & 33 & 39 & $0.81 b$ \\
\hline
\end{tabular}

Dosing and pharmacokinetic parameters of included patient are presented. Differences in categorical variables were expressed as numbers and percentages, and continuous variables as means and standard deviations if normally distributed or as medians and range between parentheses if not normally distributed. Abbreviations are: Css, plateau serum concentration; Clvanco, vancomycin clearance; AUC0-24h, area-under-the-concentration-time-curve of the first 24 hours.

a Student's t-test

b Chi-squared test

c Mann-Whitney-U test

Analysis of prospective data showed that rates of patients with a Css level $>15.0 \mathrm{mg} / \mathrm{L}$ were 69 and $63 \%$ and an AUC >350 mg. $\mathrm{kg} \cdot \mathrm{L}-1$ were 72 and $80 \%$ and for the intensive care unit and general wards populations, respectively. A significant correlation $(r 2=0.35, p=0.02)$ between predicted and observed Css levels was found. Mean predicted (18.9 $\mathrm{mg} / \mathrm{L}, 95 \% \mathrm{Cl} 18.6-19.3)$ and observed (17.4 mg/L, 95\% Cl 16.2-18.9) values were comparable. Mean bias was $-1.2 \mathrm{mg} / \mathrm{L}$ (95\% limits of agreement $0.50-1.88$ ). In both groups one patient had a drug level over $28.0 \mathrm{mg} / \mathrm{L}$. Finally, multivariate binary logistic regression analysis showed that an MDRD <40 (OR = 3.8 [1.2-11.8], $p<0.001)$ was the only parameter predictive of underexposure. After dosage correction based on linear pharmacokinetics $89 \%$ of patients' Css levels at 72 hours were within the predefined target range. 


\section{Discussion}

Ideally, a 'population-tailored' dosing algorithm for continuous vancomycin dosing is designed and prospectively validated based on historical within-population PK data. Therefore, the primary aim of our study was to describe an easy method to design and prospectively validate an algorithm for initial dosing of vancomcyin Col to facilitate the switch from intermittent to continuous dosing. Albeit the presented dosing table is in good agreement with the only other eGFR-derived dosing nomogram published by Pea et al. (17), we advice clinical centers to prospectively validate any dosing algorithm adopted from literature and recommend designing a 'population-tailored' algorithm if historical data and pharmacological knowledge are available. In addition to the data presented by Pea et al. we evaluated target attainment using the AUC as exposure parameter, since it is believed to be the major predictor with regard to efficacy. Furthermore, the nowadays more common MDRD formula was selected to estimate GFR instead of the Cockcroft\&Gault formula. Remarkably, but also in accordance with results reported by Pea et al. the performance of the dosing nomogram with regard to reaching target serum concentrations was comparable between intensive care unit and general ward populations. Target AUC attainment rates were over $70 \%$ in both populations, therefore in the remaining $30 \%$ of patients exposure could be considered suboptimal. Yet, 100 and $90 \%$ of patients had an exposure above 200 and $300 \mathrm{mg}^{*} \mathrm{~h} / \mathrm{L}$ respectively (Figure 1). Vancomycin will most likely be able to inhibit the growth of most pathogens at this exposure level during a short time period. Swift dose adjustments will optimize exposure and assure efficacy.

To rapidly achieve target trough concentrations and high tissue levels recent IDSA guidelines recommend a loading dose of $25-30 \mathrm{mg} / \mathrm{kg}$ in serious MRSA infections, including pneumonia and bacteremia (4). With respect to this recommendation our loading dose used may be too low and could be considered a limitation of the presented study next to scarce PK sampling, assumed steady state and stable vancomycin clearance, which are shortcomings faced by the majority of studies in this field and difficult to avoid due to the observational design. However, the loading dose recommendations of the IDSA guidelines are supported by limited safety data and further evaluation, primarily of the nephrotoxic effect is warranted (28).

The nephrotoxic potential of enhanced dosing of vancomycin is un- 
known for patients with severe infections who are at risk of acute kidney failure. To our knowledge the only published PK data on these intensive dosing protocols of Col of vancomycin in a clinical study have been published by Roberts et al. (29). They found a $35 \mathrm{mg} / \mathrm{kg}$ loading dose followed by high maintenance dosages of $7-14 \mathrm{mg} / \mathrm{kg} / 24 \mathrm{~h}$ was necessary to rapidly achieve $20 \mathrm{mg} / \mathrm{L}$ vancomycin concentrations in intensive care unit patients. Nevertheless, the proposed dosing protocol was based on data generated by Monte Carlo simulations and has not been prospectively validated. Recently, Ampe et al. and Schmelzer et al. tested dosing nomograms for vancomycin continuous infusion aiming for plateau' concentrations between $25-30 \mathrm{mg} / \mathrm{L}$ using higher loading doses in patients suffering from difficult-to-treat infections and 20-30 mg/L in critically ill patients respectively. They found that nephrotoxicity potentially related to vancomycin was observed in up to $25 \%$ of patients. Further research on the relation between vancomycin exposure and nephrotoxicity is urgently needed. For now the risk-benefit-ratio of 'enhanced' vancomycin dosing remains to be elucidated. When our study was designed, the IDSA guidelines had not been published yet, and a $1000 \mathrm{mg}$ loading dose for a patient of $70 \mathrm{~kg}(15 \mathrm{mg} / \mathrm{kg})$ was considered adequate at that moment $(19,30)$. Finally, our target Css levels were set at $15-20 \mathrm{mg} / \mathrm{L}$, since in our center vancomycin is primarily used to target micro-organisms with MIC's in the lower range, which is not uncommon in Northwestern European countries. Globally, vancomycin is frequently used again MRSA with higher MIC's urging for higher Css levels. Using the here presented methodology this can readily be achieved by elevating the target levels during the design and validation of any dosing algorithm.

Previous studies have shown that the MDRD tends to be less accurate in the higher and lower GFR range (26), which may explain why patients with an MDRD $<40 \mathrm{~mL} / \mathrm{min}$ are at higher risk of underexposure. However, its accuracy and precision are comparable to eGFR values estimated with the Cockcroft \& Gault formula (31). Levey and co-workers showed the MDRD formula can provide a good estimate of GFR values fro 60 to $90 \mathrm{ml} / \mathrm{min} / 1.73 \mathrm{~m} 2$ with just slightly higher bias (2.5 versus $5.5 \mathrm{~mL} / \mathrm{min}$ per $1.73 \mathrm{~m}(2))$ compared to the Chronic Kidney Disease Epidemiology Collaboration (CKD-EPI) equation, which is currently considered to to provide the most accurate GFR estimate based on serum creatinine (32).

The MDRD formula was selected, since there is worldwide trend in clinical information systems automatically to report MDRD-derived eGFR 
in patients' clinical records by clinical chemistry departments $(33,34)$. The major advantage of the MDRD over e.g. Cockcroft \& Gault formula is that it does not require the input of data on the patient's weight and therefore can be automatically calculated from patient's age, gender, race and serum creatinine. Naturally, the MDRD formula also has its shortcomings. It tends to overestimate the actual creatinine clearance in patients that are bedridden and is based on a fixed body surface area of $1.73 \mathrm{~m} 2$. A secondary explanation for higher percentage of patients with MDRD values in the lower range not reaching the predefined exposure targets is provided by Rodvold et al. who showed that the apparent volume of distribution per kg body weight in patient with an impaired renal function was proximately $30 \%$ higher increasing the risk of underexposure (35). Still, as shown by De Waele et al. a weight-based loading dose followed by a standard maintenance dose results in reasonable target attainment rates in critically ill patients (36). In theory an a priori dosing strategy of a weight-based loading dose followed by an eGFR maintenance dose has the best chance of reducing interpatient exposure variability and warrant testing in future studies $(37,38)$. In the near future serum cystatin $C$ concentration may serve as a better predictor of vancomycin clearance (39).

Apart from the ongoing debate on optimal vancomycin dosing and levels in clinically infected patients on Col, it is important to note that the IDSA expert panel advises against administration of vancomycin as continuous infusion given that a clear benefit in clinical outcomes over intermittent dosing has not been demonstrated (4). In contrast, based on PK/PD considerations Col may be the preferred mode of administration, whilst it is widely accepted that efficacy is best predicted by the AUC/MIC model. Considering vancomycin Css levels during Col can easily be converted to AUC values under steady state conditions and are associated with little AUC24h-variability as opposed to trough level monitoring in intermittent dosing. This straightforward translation of Css to AUC's is, however, not applicable to the first 24 hours following initiation of $\mathrm{Col}$, since then AUC values in individual patients are strongly affected by the loading dose and the volume of distribution. This is the main reason for using a PK model instead of an eGFR-derived linear algorithm to design the dosing table.

For current clinical practice, we propose 2 strategies depending on local availability of TDM services to obtain optimal exposure. If a TDM service is available, we advice to draw samples 2 and 8-14h after initiation of therapy. Using a PK model and Bayesian based PK soft- 
ware the plateau concentration at $24 \mathrm{~h}$ as well as the AUCO-24h can be estimated and the dose can swiftly be adjusted. If these PK 'tools' are not present, we advice to draw the first sample approximately 24 hours but not later than $36 \mathrm{~h}$ after initiation of therapy balancing between rapid dose adjustment and reaching steady state conditions.

\section{Conclusions}

An easy method to design and validate an eGFR-derived dosing schedule for continuous infusion of vancomycin was presented. Prospective validation showed good precision and accuracy in clinical practice.

\section{Funding}

This work was not supported by outside funding. All work was funded by our department research budget.

\section{Transparency declarations}

None of the authors has conflicts of interest to declare. 


\section{References}

1. Moise-Broder PA, Forrest A, Birmingham MC, Schentag JJ. Pharmacodynamics of vancomycin and other antimicrobials in patients with Staphylococcus aureus lower respiratory tract infections. Clin Pharmacokinet 2004;43(13):925-42.

$2 . \quad$ Rybak MJ. The pharmacokinetic and pharmacodynamic properties of vancomycin. Clin Infect Dis 2006;42 Suppl 1:S35-9.

3. Tenover FC, Biddle JW, Lancaster MV. Increasing resistance to vancomycin and other glycopeptides in Staphylococcus aureus. Emerg Infect Dis 2001;7(2):327-32. 4. Liu C, Bayer A, Cosgrove SE, Daum RS, Fridkin SK, Gorwitz RJ, et al. Clinical practice guidelines by the infectious diseases society of america for the treatment of methicillin-resistant Staphylococcus aureus infections in adults and children: executive summary. Clin Infect Dis 2011;52(3):285-92.

5. Rybak M, Lomaestro B, Rotschafer JC, Moellering R, Jr., Craig W, Billeter M, et al. Therapeutic monitoring of vancomycin in adult patients: a consensus review of the American Society of Health-System Pharmacists, the Infectious Diseases Society of America, and the Society of Infectious Diseases Pharmacists. Am J Health Syst Pharm 2009;66(1):82-98.

6. Hidayat LK, Hsu DI, Quist R, Shriner KA, Wong-Beringer A. High-dose vancomycin therapy for methicillin-resistant Staphylococcus aureus infections: efficacy and toxicity. Arch Intern Med 2006;166(19):2138-44.

7. Pea F, Viale P. Pharmacodynamics of antibiotics to treat multidrug-resistant Gram-positive hospital infections. Expert Rev Anti Infect Ther 2007;5(2):255-70.

8. Rybak MJ, Lomaestro BM, Rotschafer JC, Moellering RC, Craig WA, Billeter $M$, et al. Vancomycin therapeutic guidelines: a summary of consensus recommendations from the infectious diseases Society of America, the American Society of Health-System Pharmacists, and the Society of Infectious Diseases Pharmacists. Clin Infect Dis 2009;49(3):325-7.

9. Cataldo MA, Tacconelli E, Grilli E, Pea F, Petrosillo N. Continuous versus intermittent infusion of vancomycin for the treatment of Gram-positive infections: systematic review and meta-analysis. J Antimicrob Chemother. 2012;67(1):17-24.

10. van Maarseveen EM, Man WH, Touw DJ, Bouma AW, van Zanten AR. Continuous and intermittent infusion of vancomycin equally effective: review of the literature. Ned Tijdschr Geneeskd. 2011;155(42):A2667.

11. Schmelzer TM, Christmas AB, Norton HJ, Heniford BT, Sing RF. Vancomycin intermittent dosing versus continuous infusion for treatment of ventilator-associated pneumonia in trauma patients. Am Surg;79(11):1185-90.

12. Raverdy V, Ampe E, Hecq JD, Tulkens PM. Stability and compatibility of vancomycin for administration by continuous infusion. J Antimicrob Chemother;68(5):1179-82.

13. Bernard L, El H, Pron B, Lotthe A, Gleizes V, Signoret F, et al. Outpatient parenteral antimicrobial therapy (OPAT) for the treatment of osteomyelitis: evaluation of efficacy, tolerance and cost. J Clin Pharm Ther 2001;26(6):445-51.

14. van Zanten AR, Engelfriet PM, van Dillen K, van Veen M, Nuijten MJ, Polderman $\mathrm{KH}$. Importance of nondrug costs of intravenous antibiotic therapy. Crit Care 2003;7(6):R184-90.

15. Boffi El Amari E., Vuagnat A., Stern R., Assal M., Denormandie P., Hoffmeyer P., Bernard L. High versus standard dose vancomycin for osteomyelitis. Scand J Infect Dis 2004;36(10):712-7. 
16. Hutschala D, Kinstner C, Skhirdladze K, Thalhammer F, Muller M, Tschernko E. Influence of vancomycin on renal function in critically ill patients after cardiac surgery: continuous versus intermittent infusion. Anesthesiology 2009;111(2):356-65.

17. Pea F, Furlanut M, Negri C, Pavan F, Crapis M, Cristini F, et al. Prospectively validated dosing nomograms for maximizing the pharmacodynamics of vancomycin administered by continuous infusion in critically ill patients. Antimicrob Agents Chemother 2009;53(5):1863-7.

18. Vuagnat A, Stern R, Lotthe A, Schuhmacher H, Duong M, Hoffmeyer P, et al. High dose vancomycin for osteomyelitis: continuous vs. intermittent infusion. J Clin Pharm Ther 2004;29(4):351-7.

19. Wysocki M, Delatour F, Faurisson F, Rauss A, Pean Y, Misset B, et al. Continuous versus intermittent infusion of vancomycin in severe Staphylococcal infections: prospective multicenter randomized study. Antimicrob Agents Chemother 2001;45(9):2460-7.

20. Ingram PR, Lye DC, Fisher DA, Goh WP, Tam VH. Nephrotoxicity of continuous versus intermittent infusion of vancomycin in outpatient parenteral antimicrobial therapy. Int J Antimicrob Agents 2009;34(6):570-4.

21. Spapen HD, Janssen van Doorn K, Diltoer M, Verbrugghe W, Jacobs R, Dobbeleir $\mathrm{N}$, et al. Retrospective evaluation of possible renal toxicity associated with continuous infusion of vancomycin in critically ill patients. Ann Intensive Care 2011;1(1):26.

22. Bosso JA, Nappi J, Rudisill C, Wellein M, Bookstaver PB, Swindler J, et al. Relationship between vancomycin trough concentrations and nephrotoxicity: a prospective multicenter trial. Antimicrob Agents Chemother 2011;55(12):5475-9.

23. Elyasi S, Khalili H, Dashti-Khavidaki S, Mohammadpour A. Vancomycin-induced nephrotoxicity: mechanism, incidence, risk factors and special populations. A literature review. Eur J Clin Pharmacol 2012;68(9):1243-55.

24. Proost JH, Meijer DK. MW/Pharm, an integrated software package for drug dosage regimen calculation and therapeutic drug monitoring. Comput Biol Med 1992;22(3):155-63.

25. Pullen J, de Rozario L, Stolk LM, Degraeuwe PL, van Tiel FH, Zimmermann LJ. Population pharmacokinetics and dosing of flucloxacillin in preterm and term neonates. Ther Drug Monit 2006;28(3):351-8.

26. Levey AS, Bosch JP, Lewis JB, Greene T, Rogers N, Roth D. A more accurate method to estimate glomerular filtration rate from serum creatinine: a new prediction equation. Modification of Diet in Renal Disease Study Group. Ann Intern Med 1999;130(6):461-70.

27. Bellomo R, Ronco C, Kellum JA, Mehta RL, Palevsky P, Acute Dialysis Quality Initiative w. Acute renal failure - definition, outcome measures, animal models, fluid therapy and information technology needs: the Second International Consensus Conference of the Acute Dialysis Quality Initiative (ADQI) Group. Critical Care (London, England) 2004;8(4):R204.

28. Kullar R, Davis SL, Taylor TN, Kaye KS, Rybak MJ. Effects of Targeting Higher Vancomycin Trough Levels on Clinical Outcomes and Costs in a Matched Patient Cohort. Pharmacotherapy 2012;32(3):195-201.

29. Roberts JA, Taccone FS, Udy AA, Vincent JL, Jacobs F, Lipman J. Vancomycin dosing in critically ill patients: robust methods for improved continuous-infusion regimens. Antimicrob Agents Chemother 2011;55(6):2704-9. 
30. Pea F FM, Negri C, Pavan F, Crapis M, Cristini F, Viale P. Prospectively validated dosing nomograms for maximizing the pharmacodynamics of vancomycin administered by continuous infusion in critically ill patients. Antimicrob Agents Chemother. 2009;53(5):1863-7.

31. Baptista JP, Udy AA, Sousa E, Pimentel J, Wang L, Roberts JA, et al. A comparison of estimates of glomerular filtration in critically ill patients with augmented renal clearance. Crit Care 2011;15(3):R139.

32. Levey AS, Stevens LA, Schmid CH, Zhang YL, Castro AF, 3rd, Feldman $\mathrm{HI}$, et al. A new equation to estimate glomerular filtration rate. Ann Intern Med 2009;150(9):604-12.

33. Accetta NA, Gladstone EH, DiSogra C, Wright EC, Briggs M, Narva AS. Prevalence of estimated GFR reporting among US clinical laboratories. Am J Kidney Dis 2008;52(4):778-87.

34. Imbasciati E, Falbo R, Mariani P, Signorelli S, Stella A, Brambilla P. A regional survey of serum creatinine measurement methods and estimated glomerular filtration rate reporting. J Nephrol 2012:0.

35. Rodvold KA, Blum RA, Fischer JH, Zokufa HZ, Rotschafer JC, Crossley KB, et al. Vancomycin pharmacokinetics in patients with various degrees of renal function. Antimicrob Agents Chemother 1988;32(6):848-52.

36. De Waele JJ, Danneels I, Depuydt P, Decruyenaere J, Bourgeois M, Hoste E. Factors associated with inadequate early vancomycin levels in critically ill patients treated with continuous infusion. Int J Antimicrob Agents;41(5):434-8.

37. Saugel B, Nowack MC, Hapfelmeier A, Umgelter A, Schultheiss C, Thies P, et al. Continuous intravenous administration of vancomycin in medical intensive care unit patients. J Crit Care;28(1):9-13.

38. Ocampos-Martinez E PL, Scolletta S, Abdelhadii A, Devigili A, Cianferoni S, de Backer D, Jacobs F, Cotton F, Vincent JL, Taccone FS. Determinants of early inadequate vancomycin concentrations during continuous infusion in septic patients. Int $J$ Antimicrob Agents 2012;39(4):332-7.

39. Chen YC, Feng JF, Li B, Zhang L, Yang YW. Estimation of safe and effective dose of vancomycin in MRSA-infected patients using serum cystatin $\mathrm{C}$ concentrations. Int J Clin Pharmacol Ther;51(3):161-9. 


\section{Appendix 1. Brief description of the design and validation of the pharmacokinetic model}

Manuscript title: 'Exposure variability and target attainment of vancomycin intermittent and continuous infusion in critically ill patients ready, aim, target locked?'

\# corresponding author:

Erik M. van Maarseveen

University Medical Center Utrecht

Department of Clinical Pharmacy, Room HP D00.218

Heidelberglaan 100

P.O.Box 85500, 3508 GA Utrecht The Netherlands

Telephone:

$+31-88-7557218$

Fax:

$+31-88-7556756$

Email: $\quad$ E.M.vanmaarseveen@UMCUtrecht.nl

\section{Model design}

Data were collected from patient clinical records, therapeutic drug monitoring (TDM) database and the electronic prescription database. A pharmacokinetic population model was designed using the KinPop module of MWPharm (version 3.60, MedilWare, Zuidhorn, the Netherlands) and historical within-population data obtained from 18 surgical ward patients, 22 internal ward patients and 55 intensive care unit patients on vancomycin intermittent infusion. MWPharm uses an iterative two-stage Bayesian approach to estimate the mean and coefficient of variation of relevant pharmacokinetic parameters. Population models were designed and validated according to previously published methodology [1]. The following polynomial formula was used to estimate the SD (standard deviation) of the analytical assay error: $\mathrm{SD}=0.323$ $+0.0265{ }^{*} \mathrm{C}+0.0002{ }^{*} \mathrm{C}$. A two-compartment model was designed. 
The elimination rate constant was defined by the formulae: Kel $=$ Kelm + Kelr and Kelr $=$ Kslope ${ }^{*} \mathrm{Clcr}$, in which Kelm and Kelr represent the metabolic and renal elimination constant, respectively. The glomular filtration rate of vancomycin was assumed to be constant. The model was evaluated by bootstrap analysis ( $n=1000$ replicates), an analysis of individual predicted versus observed concentrations, an analysis of the residual error versus the observed concentrations and model fit was checked visually for all individual patients. A pharmacokinetic model was established. Bootstrap analysis resulted similar parameters estimates (Table A1). Predicted versus observed serum concentrations plot showed good correlation $(r 0.87, p<0.01)$ and weighted residuals were randomly distributed over the concentration range. Visual check of patients' concentration-versus-time-curves showed good individual fits.

\begin{tabular}{lllll}
\hline & PK model parameters & & \multicolumn{2}{l}{ Bootstrap estimates } \\
& Estimate & CV (\%) & Mean & RSE (\%) \\
\hline & 0.018 & 225 & 0.022 & 278 \\
Kelm (h-1) & 0.0037 & 31 & 0.0034 & 17 \\
Kslope (h-1/(ml min-1)) & 0.29 & 29 & 0.32 & 15 \\
V1 (L/kg) & 0.92 & 47 & 0.61 & 15 \\
K12 (h-1) & 0.46 & 116 & 0.38 & 45 \\
K21 (h-1) & & &
\end{tabular}

Table A1. Pharmacokinetic population estimates and bootstrap analysis results of Inl model in critically ill patients. Abbreviations: ICU, intensive care unit; Inl, intermittent infusion; Kelm, metabolic elimination rate constant; Kslope, renal elimination rate constant per $\mathrm{ml} / \mathrm{min}$ clearance; PK, pharmacokinetic; CV, coefficient of variation; RSE, relative standard error; $\mathrm{V} 1$, volume of distribution in the central compartm

References

1. Pullen, J., L. de Rozario, L.M. Stolk, P.L. Degraeuwe, F.H. van Tiel and L.J. Zimmermann (2006) Population pharmacokinetics and dosing of flucloxacillin in preterm and term neonates, Ther Drug Monit 28: 351-358 


\section{Chapter 4}

Summary, clinical implications and future perspectives

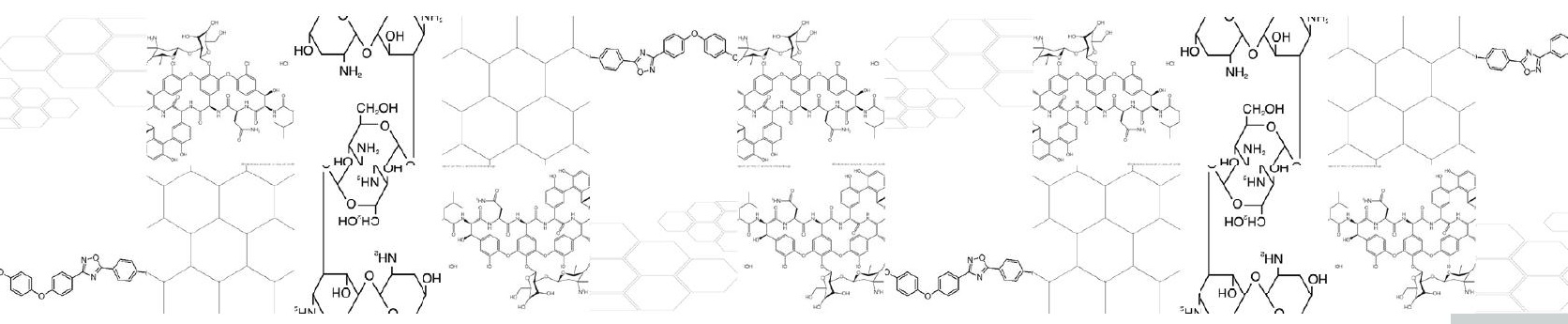



In this thesis we have investigated pharmacokinetically based strategies for optimization of aminoglycoside and vancomycin therapies. The current chapter will summarize and discuss the results of our studies investigating aminoglycoside and vancomycin pharmacokinetics in the studied patient populations with infections.

\section{Once daily and extended interval dosing of aminoglycosides}

As once daily dosing of aminoglycosides has gained popularity worldwide, more data are available on once daily dosing regimens in various clinically infected populations. In the study described in Chapter 2.1 we focused on adult cystic fibrosis (CF) patients. CF patients are frequently exposed to high doses of once daily dosed (ODD) tobramycin with relatively long treatment course duration in case of pulmonary exacerbations (1). The tobramycin dosages range from 10 to $15 \mathrm{mg} / \mathrm{kg}$ once daily in this population. Based on harmacokinetic/pharmacodynamic (PK/PD) considerations a high dose per kg bodyweight is a consequence of the relatively large volume of distribution and high clearance in comparison to other clinically infected populations treated with aminoglycosides (2). Current once daily high tobramycin dosing protocols in pediatric and adult CF patient populations result in high peak and low trough concentrations ensuring efficacy and limiting the risk of toxicity, respectively (3). In Chapter 2.1 the influence of the time of administration on the PK of tobramycin in adult CF patients was investigated. This study was designed to test the assumption that dosing in the evening results in a lower clearance and an increased area under the concentration-time curve (AUC) of aminoglycosides due to the circadian rhythm of renal function. In close collaboration, the effect of the circadian rhythm on tobramycin PK was studied in pediatric CF patients by investigators at the University of Nottingham, UK. Results from the latter studies indicate no clinically relevant effect of the administration time on tobramycin PK in CF patients. In Chapter 2.2 we performed a retrospective cohort study that investigated the effect of the time of administration of aminoglycosides in general ward patients and in intensive care unit (ICU) patients. In these clinically infected populations also no effects of the time of administration could be detected on the PK of aminoglycosides. Furthermore, the incidence of nephrotoxicity was not affected by the time of administration in the general ward population. We suggest that the lack of an effect of the time of administration on 
aminoglycoside PK in CF patients, general ward patients and ICU patients is caused by a distortion of the 'normal' circadian rhythm in these clinically infected patients. Previous reports have demonstrated that changes in immunological status affect the circadian rhythm, e.g. sepsis will abrogate the normal melatonin secretion over the day (4-6). The presence of a 'normal' circadian rhythm is influenced by distortion of immunological homeostasis. In fact, based on our observations we hypothesize that intravenous antibiotic agents will rarely show a diurnal pattern since they are prescribed to patients with serious suspected or proven infections.

In Chapter 2.3 we investigated the PK and nephrotoxicity of ODD gentamicin and ODD tobramycin in a large retrospective cohort of infected general ward patients. The most important finding of this study was a fifty percent lower incidence of nephrotoxicity in patients treated with tobramycin compared to those treated with gentamicin. To our knowledge no previous studies have compared the clinical toxicity of two or more ODD aminoglycosides. Importantly, this study offers a pharmacological explanation for the difference in nephrotoxicity between gentamicin and tobramycin, since the PK analysis showed that gentamicin clearance was lower than tobramycin clearance. As a consequence, gentamicin exposure in terms of daily AUC was higher compared to tobramycin exposure. Mechanistically, our findings can be explained by the higher renal accumulation of gentamicin versus tobramycin inducing renal injury (7). Finally, a multivariate analysis showed that the AUC of the first administration was a strong predictor of the incidence of nephrotoxicity indicating 'early therapeutic drug monitoring (TDM)' using limited AUC sampling can be of additive value identifying those patients at risk and therefore is of clinical relevance.

In Chapter 2.4 we prospectively evaluated a gentamicin dosing regimen which is used in newborns with suspected or proven neonatal sepsis. This study showed that a dosing regimen of $5 \mathrm{mg} / \mathrm{kg}$ every $36 \mathrm{~h}$ resulted in therapeutic exposure of over $90 \%$ of included subjects. It is important to note that this study was not performed in a neonatal intensive care unit (NICU) but in a level 2 special care nursery population. In accordance with previous reports (8), we found a strong relationship between the volume of distribution $(\mathrm{L} / \mathrm{kg})$ of gentamicin with bodyweight in our population, with a larger bodyweight-corrected volume of distribution in preterm infants due to differences in body composition. Gentamicin half-life decreased with lower gestational age, which has also been reported by others (9). A combination of low weight-corrected 
volume of distributions with short half-lives will translate in concentration-vs-time-curves with high peak concentrations and steep slopes.

Vice versa, in low-birth-weight premature newborns peak concentrations and half-lives were lower and longer, respectively. Interestingly, the AUC was not related to gestational age or body weight, meaning that using the proposed dosing regimen in low birth-weight more premature newborns on average had the same gentamicin exposure compared to older neonates with higher bodyweights.

\section{Continuous infusion of vancomycin}

In Chapter 3.1 we performed a review of the literature comparing the inter-patient exposure variability in clinically infected patients on continuous or intermittent infusion of vancomycin. This review showed that continuous infusion conferred a fifty percent lower inter-patient variability of vancomycin concentrations compared to intermittent infusion. This interesting finding based on the review of in total 34 published reports led to the design of a retrospective cohort study in an ICU population (Chapter 3.2). In this study we confirmed the hypothesis that the inter-patient variability in vancomycin exposure was significantly lower for continuously dosed versus the intermittently dosed critically ill patients. Furthermore, 'plateau' concentrations in continuous dosing showed a stronger correlation with estimated AUCs compared to trough concentrations during intermittent dosing. Two conveyable causes can largely explain the reported results in Chapter 3.1 and 3.2. First, since 'trough sample timing' can have a major influence on the measured vancomycin concentrations, an intermittent dosing regimen monitored by trough concentration is inherently prone to higher variability in comparison to continuous infusion and 'plateau' concentration monitoring (10).

Second, when trough concentrations are monitored in case of intermittent dosing the variability of the AUC is not only affected by the vancomycin clearance, but also by the volume of distribution (Chapter 3.1 and 3.2). Patients with a relatively high volume of distribution will need higher intermittent dosages to maintain trough concentrations within the therapeutic range resulting in a large 'delta' between peak and trough concentrations and therefore in high AUCs, especially in once or twice daily dosing regimens. In Chapter 3.3 a simple method was presented to clinically implement a vancomycin continuous dosing nomogram based on historical within-population data obtained from 
intermittently dosed patients. This chapter offers tools for clinicians considering a switch from intermittent to continuous dosing of vancomycin. Although not intended for use in other clinically infected populations the developed dosing nomogram was in accordance with the only other prospectively validated dosing algorithm (11).

\section{Implications for clinical practice}

In chapters 2.1 and 2.2 we showed that the time of administration did not affect the clearance or exposure in terms of AUC in CF patients, general ward patients and ICU patients. Hence, swifting administration times to the morning period is not recommended and 24 hour intervals should be maintained unless therapeutic drug monitoring results indicate that the dosing interval needs to be extended (12). Furthermore, since early initiation of antibiotic treatment is paramount to improve outcomes, the first administration of an aminoglycoside should take place as soon as possible after the diagnosis (13).

Although our findings deserve to be confirmed, results from Chapter 2.3 show a higher nephrotoxic potential of ODD gentamicin over ODD tobramycin in clinically infected patients treated for at least 3 days which may lead to the revision of (inter)national and local treatment protocols. In patients who are at high risk of developing nephrotoxicity and/or are likely to be treated over a longer period, tobramycin should be preferred over gentamicin. In fact, European cystic fibrosis treatment guidelines already advocate to choose tobramycin over gentamicin for this reason $(14,15)$. The same concerns with regard to cumulative toxicity apply to other highly exposed populations, e.g. patients treated for endocarditis. In order to optimize treatment with aminoglycosides 'early' AUC monitoring in addition to 'trough levels' should be implemented in daily routine, since our data suggest the AUC of the first administration is a strong predictor of nephrotoxicity.

The added value to the present literature of our study results presented in Chapter 2.4 on gentamicin dosing in neonates lies in the specific neonatal population investigated. Current dosing regimens in neonates are largely based on data obtained from neonates admitted to a NICU $(9,16,17)$, while the number of neonates exposed to gentamicin is likely to be higher in level 2 special care nurseries. Albeit our data deserve to be prospectively confirmed in a second independent cohort of neonates admitted to a level 2 special care nursery, our findings show that a more uniform dosing protocol of $5 \mathrm{mg} / \mathrm{kg}$ per 36 hours can be implemented in level 2 unit compared to more complex dosing protocols proposed in neonates admitted to a level 3 intensive care unit (16, 18, 19). As described in Chapter 3.1 vancomycin continuous dosing 
protocols are rapidly gaining interest and are being implemented in clinical practice. Apart from some practical advantages over intermittent infusion, continuous infusion of vancomycin shows a more predictable exposure and thus the odds of an optimal target attainment as shown in Chapter 3.1 and 3.2 are better. In addition, continuous infusion of vancomycin might also be preferred since the drug preparation time, the staff workload and costs are reduced and it may also shorten hospital length of stay. $(20,21),(22)$ and has shown to be safe concerning stability (23). Of course, there are also potential disadvantages to continuous infusion. Sufficient numbers of volume controlled infusion pump systems have to be secured and compatibility with other simultaneously infused drugs can be an issue. Nevertheless, 'Y-site'-compatibility of vancomycin with many drugs has been demonstrated (24). Finally, based on our methodology described in Chapter 3.2 a 'tailored' dosing algorithm of vancomycin can be designed for clinically infected populations aiming to achieve a priori target exposure using historical within-population data, a PK model and PK software.

\section{Suggestions for future studies}

Toxicity studies in populations treated with 'traditional' dosing regimens of aminoglycosides or vancomycin have been performed in the past. However, limited data are available on the recommended dosing regimens from most recent guidelines with respect to clinical outcomes $(25,26)$. For example, the most recent treatment guidelines for the treatment of multi-resistant S. aureus (MRSA) of the Infectious Diseases Society of America (IDSA) promoting high exposure targets for vancomycin are sparsely supported by safety data (27). Moreover, most toxicity studies on aminoglycosides were performed in the multiple daily dosing era. Therefore, prospective studies investigating the toxicity of currently prescribed once daily or extended-interval dosed aminoglycosides in highly exposed populations e.g. patients suffering from endocarditis are warranted. Newly developed high dosing protocols of vancomycin for long-term MRSA treatment also raise questions about the risk/benefit ratio (28).

Furthermore, there is a need for pharmacokinetic studies investigating vancomycin exposure in patients on continuous infusion in detail during the first 24 hours of therapy (29-31). Therefore, we advocate future studies to address this issue first by incorporating intensive 
PK monitoring during the first 24 hours to design proper PK models of continuous infusion of vancomycin and identify optimal sampling times for limited sampling strategies in clinically infected populations. Secondly, determinants for patients at risk of under- or overexposure may be identified. Next, we recommend the implementation of 'early TDM', which has recently also been suggested for intermittent dosing of vancomycin (32). The AUC24h and steady state concentation can be estimated accurately shortly after initiation of vancomycin from two serum samples drawn within 12 hours after initiation of therapy using a validated PK model and PK software. This 'early TDM' approach offers the opportunity to swiftly identify patients that are or will be significantly under- or overexposed and may thereby enable clinicians to improve patient outcomes. Finally, the ongong debate on the preferential mode of administration of vancomycin should be addressed by large multicenter investigations preferably of prospective randomized design.

Many experts believe that clinical usage of tobramycin and gentamicin will increase due to resistance to our current first line antibiotic armamentarium (33-36). This could result in selection pressure of first choice aminoglycosides on a global scale. Recently, the Swedish Reference Group for Antibiotics (SRGA) has carried out a risk-benefit analysis of aminoglycoside treatment based on clinical efficacy, antibacterial spectrum, and synergistic effect with beta-lactam antibiotics, endotoxin release, toxicity, and side effects. For instance, in cases of suspected infection caused by multidrug-resistant Enterobacteriaceae the SRGA nowadays promotes amikacin instead of gentamicin or tobramycin which is generally more active against extended-spectrum beta-lactamase (ESBL)-producing and quinolone-resistant Escherichia coli than other aminoglycosides (37).

Of interest, after the introduction of the SRGA guidelines the increase in Swedish orders of amikacin immediately lead to drug shortages in other EU countries. This is just one examples showing that antimicrobial agent shortages are of global concern and not limited to second or third world countries nowadays (38). It is a multifactorial problem and the current situation of antibiotic shortages is unfortunately more likely to aggravate than to disappear in the years to come.

With regard to the amikacin nephrotoxic and ototoxic potential, there is mechanistic and clinical evidence that the risk of developing nephrotoxicity is relatively low compared to the others within-class drugs (39, 40). Nevertheless, more studies on amikacin with current once daily 
dosing regimens are needed. For similar reasons, netelmicin, a previously used aminoglycoside which is though to have a low nephrotoxic potential $(40,41)$, will regain interest. It is also worthwhile to mention plazomicin, a promising aminoglycoside, that has been tested in phase 3 studies (42). Plazomicin is designed to overcome tobramycin and gentamicin resistance. Human studies to date have not yet reported nephrotoxicity or ototoxicity, but lack of plazomicin ototoxicity has been reported in the guinea pig model for plazomicin (42). Given the reported increase in bacterial resistance to current antimicrobial agents, plazomicin may be considered a welcome addition to the antibacterial armamentarium pending positive results from large-scale clinical trials and other required clinical studies. In conclusion PK/PD studies of 'new' dosing regimens of 'older' aminoglycoside antibiotics are urgently needed, anticipating broad clinical use of these antimicrobal agents in patient populations with serious infections and a high drug exposure. 


\section{References}

1. Tiddens HA. Detecting early structural lung damage in cystic fibrosis. Pediatr Pulmonol. 2002;34(3):228-31.

2. Touw DJ, Vinks AA, Neef C. Pharmacokinetic modelling of intravenous tobramycin in adolescent and adult patients with cystic fibrosis using the nonparametric expectation maximization (NPEM) algorithm. Pharm World Sci. 1997;19(3):142-51.

3. Touw DJ, Vinks AA, Heijerman HG, Hermans J, Bakker W. Suggestions for the optimization of the initial tobramycin dose in adolescent and adult patients with cystic fibrosis. Ther Drug Monit. 1994;16(2):125-31.

4. Mundigler G, Delle-Karth G, Koreny M, et al. Impaired circadian rhythm of melatonin secretion in sedated critically ill patients with severe sepsis. Crit Care Med 2002;30:536-540.

5. Verceles AC, Silhan L, Terrin M, Netzer G, Shanholtz C, Scharf SM. Circadian rhythm disruption in severe sepsis: the effect of ambient light on urinary 6-sulfatoxymelatonin secretion. Intensive Care Med 2012;38(5):804-10.

6. Habbal OA, Al-Jabri AA. Circadian rhythm and the immune respons: a review. It Rev Immunol 2009;28:93-108.

7. Houghton DC, Plamp CE, 3rd, DeFehr JM, Bennett WM, Porter G, Gilbert D. Gentamicin and tobramycin nephrotoxicity. A morphologic and functional comparison in the rat. Am J Pathol 1978;93(1):137-52.

8. Pacifici GM. Clinical pharmacokinetics of aminoglycosides in the neonate: a review. Eur J Clin Pharmacol 2009;65(4):419-27.

9. Touw DJ, Westerman EM, Sprij AJ. Therapeutic drug monitoring of aminoglycosides in neonates. Clin Pharmacokinet 2009;48(2):71-88.

10. Roberts JA, Lipman J. Antibacterial dosing in intensive care: pharmacokinetics, degree of disease and pharmacodynamics of sepsis. Clin Pharmacokinet 2006;45(8):755-73.

11. Pea F, Furlanut M, Negri C, Pavan F, Crapis M, Cristini F, et al. Prospectively validated dosing nomograms for maximizing the pharmacodynamics of vancomycin administered by continuous infusion in critically ill patients. Antimicrob Agents Chemother 2009;53(5):1863-7.

12. Kahlmeter G, Dahlager JI. Aminoglycoside toxicity - a review of clinical studies published between 1975 and 1982. J Antimicrob Chemother 1984;13 Suppl A:9-22.

13. Kumar A, Roberts D, Wood KE, Light B, Parrillo JE, Sharma S, et al. Duration of hypotension before initiation of effective antimicrobial therapy is the critical determinant of survival in human septic shock. Crit Care Med 2006;34(6):1589-96.

14. Smyth A, Lewis S, Bertenshaw C, Choonara I, McGaw J, Watson A. Case-control study of acute renal failure in patients with cystic fibrosis in the UK. Thorax 2008;63(6):532-5.

15. Kerem E, Conway S, Elborn S, Heijerman H. Standards of care for 
patients with cystic fibrosis: a European consensus. J Cyst Fibros 2005;4(1):726.

16. Begg EJ, Vella-Brincat JW, Robertshawe B, McMurtrie MJ, Kirkpatrick CM, Darlow B. Eight years' experience of an extended-interval dosing protocol for gentamicin in neonates. J Antimicrob Chemother 2009;63(5):1043-9.

17. Thureen PJ, Reiter PD, Gresores A, Stolpman NM, Kawato K, Hall DM. Once- versus twice-daily gentamicin dosing in neonates $>/=34$ Weeks' gestation: cost-effectiveness analyses. Pediatrics 1999;103(3):594-8.

18. Hitron AE, Sun Y, Scarpace SB. Accuracy of empiric gentamicin dosing guidelines in neonates. J Pediatr Pharmacol Ther 2010;15(4):264-73.

19. Semchuk W, Borgmann J, Bowman L. Determination of a gentamicin loading dose in neonates and infants. Ther Drug Monit 1993;15(1):47-51.

20. Boffi El Amari E, Vuagnat A, Stern R, Assal M, Denormandie P, Hoffmeyer P, Bernard L. High versus standard dose vancomycin for osteomyelitis. Scand J Infect Dis 2004;36(10):712-7.

21. van Zanten AR, Engelfriet PM, van Dillen K, van Veen M, Nuijten MJ, Polderman $\mathrm{KH}$. Importance of nondrug costs of intravenous antibiotic therapy. Crit Care 2003;7(6):R184-90.

22. Bernard L, El H, Pron B, Lotthe A, Gleizes V, Signoret F, et al. Outpatient parenteral antimicrobial therapy (OPAT) for the treatment of osteomyelitis: evaluation of efficacy, tolerance and cost. J Clin Pharm Ther 2001;26(6):44551.

23. Raverdy V, Ampe E, Hecq JD, Tulkens PM. Stability and compatibility of vancomycin for administration by continuous infusion. J Antimicrob Chemother.

24. LA T. Trissel's Handbook on injectable drugs. 4th edition 2009.

25. Munckhof WJ, Grayson ML, Turnidge JD. A meta-analysis of studies on the safety and efficacy of aminoglycosides given either once daily or as divided doses. J Antimicrob Chemother 1996(37):4.

26. Hazlewood KA, Brouse SD, Pitcher WD, Hall RG. Vancomycin-associated nephrotoxicity: grave concern or death by character assassination? Am J Med 2010;123(2):182 e1-7.

27. Rybak MJ, Lomaestro BM, Rotschafer JC, Moellering RC, Craig WA, Billeter M, et al. Vancomycin therapeutic guidelines: a summary of consensus recommendations from the infectious diseases Society of America, the American Society of Health-System Pharmacists, and the Society of Infectious Diseases Pharmacists. Clin Infect Dis 2009;49(3):325-7.

28. Bosso JA, Nappi J, Rudisill C, Wellein M, Bookstaver PB, Swindler $\mathrm{J}$, et al. Relationship between vancomycin trough concentrations and nephrotoxicity: a prospective multicenter trial. Antimicrob Agents Chemother 2011;55(12):5475-9.

29. Roberts JA, Taccone FS, Udy AA, Vincent JL, Jacobs F, Lipman J. 
Vancomycin dosing in critically ill patients: robust methods for improved continuous-infusion regimens. Antimicrob Agents Chemother 2011;55(6):2704-9. 30. Liu C, Bayer A, Cosgrove SE, Daum RS, Fridkin SK, Gorwitz RJ, et al. Clinical practice guidelines by the infectious diseases society of america for the treatment of methicillin-resistant Staphylococcus aureus infections in adults and children: executive summary. Clin Infect Dis 2011;52(3):285-92.

31. van Maarseveen EM. Continuous infusion of vancomycin: effective, efficient and safe [Abstract]. In: 12th Congress of Therapeutic Drug Monitoring \& Clinical Toxicology. Stuttgart: IATDMCT 2011; 2011. p. p 514. Abstract p131. 32. Jones TE, Vasileff $\mathrm{H}$, Hewton $\mathrm{C}$. Should monitoring of vancomycin be delayed? A case of likely nephrotoxicity occasioned by morbid obesity and minimal monitoring. Br J Clin Pharmacol 2012;74(6):1063-5.

33. Poonsuk K, Tribuddharat $\mathrm{C}$, Chuanchuen R. Aminoglycoside resistance mechanisms in Pseudomonas aeruginosa isolates from non-cystic fibrosis patients in Thailand. Can J Microbiol 2013;59(1):51-6.

34. Dubois V, Arpin C, Dupart V, Scavelli A, Coulange L, Andre C, et al. Beta-lactam and aminoglycoside resistance rates and mechanisms among Pseudomonas aeruginosa in French general practice (community and private healthcare centres). J Antimicrob Chemother 2008;62(2):316-23.

35. NETHMAP. Consumption of antimicrobial agents and antimicrobial resistance among medically important bacteria in the Netherlands. Stichting Werkgroep Antibiotica Beleid en RIVM 2010.

36. Gould IM, Bal AM. New antibiotic agents in the pipeline and how they can help overcome microbial resistance. Virulence;4(2):185-91.

37. Hanberger $\mathrm{H}$, Edlund C, Furebring M, C GG, Melhus A, Nilsson LE, et al. Rational use of aminoglycosides--review and recommendations by the Swedish Reference Group for Antibiotics (SRGA). Scand J Infect Dis;45(3):161-75.

38. Gundlapalli AV, Beekmann SE, Graham DR, Polgreen PM. Perspectives and concerns regarding antimicrobial agent shortages among infectious disease specialists. Diagn Microbiol Infect Dis;75(3):256-9.

39. De Broe ME, Giuliano RA, Verpooten GA. Choice of drug and dosage regimen. Two important risk factors for aminoglycoside nephrotoxicity. Am J Med 1986;80(6B):115-8.

40. Mingeot-Leclercq MP, Tulkens PM. Aminoglycosides: nephrotoxicity. Antimicrob Agents Chemother 1999;43(5):1003-12.

41. Contrepois A, Brion N, Garaud JJ, Faurisson F, Delatour F, Levy JC, et al. Renal disposition of gentamicin, dibekacin, tobramycin, netilmicin, and amikacin in humans. Antimicrob Agents Chemother 1985;27(4):520-4.

42. Zhanel GG, Lawson CD, Zelenitsky S, Findlay B, Schweizer F, Adam $\mathrm{H}$, et al. Comparison of the next-generation aminoglycoside plazomicin to gentamicin, tobramycin and amikacin. Expert Rev Anti Infect Ther;10(4):459-73. 



\section{Nederlandse Samenvatting}

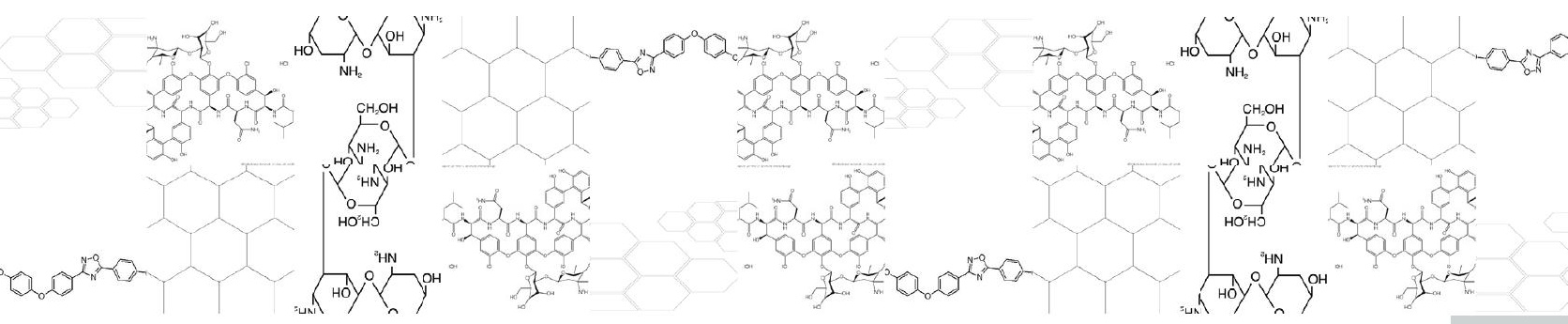


In dit proefschrift worden strategieën ter optimalisatie van de behandeling met de antibiotica aminoglycosiden en vancomycine onderzocht. De blootstelling van de individuele patiënt aan deze antibiotica was hierbij het uitgangspunt. Door het meten van geneesmiddelconcentraties in het bloed van patiënten met infecties werd voor ieder individu naar de optimale dosering gezocht. Daarbij wordt gestreefd naar een zo gunstig mogelijke balans tussen effectiviteit en veiligheid van aminoglycosiden en vancomycine.

Het inleidende hoofdstuk 1 geeft een globaal overzicht van de werkzaamheid, farmacokinetiek, effectiviteit en veiligheid van aminoglycosiden en vancomycine. In hoofdstuk 1.2 is tevens een review naar de klinische uitkomsten van intermitterende versus continue infusie van vancomycine opgenomen. De belangrijkste conclusies van deze systematische review zijn dat continue infusie minder nefrotoxisch lijkt te zijn en dat er op basis van de beschikbare data geen verschil in effectiviteit tussen beide toedieningsvormen kan worden aangetoond.

In de hoofdstukken 2.1 en 2.2 is bestudeerd of het toedienen van de dagelijkse dosis van een aminoglycoside in de ochtend versus de avond invloed had op de farmacokinetiek van gentamicine en tobramycine. Van meerdere antibiotica is een effect van het circadiaans ritme, dat wil zeggen dag-nachtritme, op de farmacokinetiek van het geneesmiddel beschreven. Doordat meerdaagse regimes van aminoglycosiden voor vrijwel alle indicaties zijn vervangen door minder nefrotoxische eenmaal daagse doseringen, zou het tijdstip van toediening van een aminoglycoside een klinisch relevant effect op de blootstelling kunnen hebben. Wij hebben dit effect bestudeerd in een met tobramycine behandelde populatie van volwassen taaislijmziektepatiënten en bij met tobramycine en gentamicine behandelde patiënten die met een infectie opgenomen werden in een ziekenhuis. In deze onderzoeken werd geen effect gevonden van het tijdstip van intraveneuze toediening van deze aminoglycosiden op de blootstelling. Tevens vonden we geen aanwijzingen voor klinisch relevante verschillen in nefrotoxiciteit. De meest voor de hand liggende verklaring voor de gevonden resultaten is dat een patiënt met een ernstige infectie het 'normale' dag-nachtritme verliest, waardoor het tijdstip van de toediening geen effect op de blootstelling heeft. Bovenstaande studies zijn in nauwe samenwerking met onderzoekers van de Universiteit van Nottingham uitgevoerd. 
In een vergelijkbaar onderzoek bij kinderen met taaislijmziekte kwamen zij tot dezelfde conclusies.

In Hoofdstuk 2.3 onderzochten we retrospectief de farmacokinetiek en nefrotoxiciteit van een eenmaal daagse dosering gentamicine en tobramycine in een groot cohort van in het ziekenhuis opgenomen patiënten met (een verdenking op) een infectie. De belangrijkste bevinding van deze studie was dat met tobramycine behandelde patiënten een $50 \%$ lagere kans hadden op het ontwikkelen van nefrotoxiciteit in vergelijking met patiënten die gentamicine toegediend hadden gekregen. Dit onderzoek is tot op heden de enige studie die de klinische nefrotoxiciteit van twee of meer eenmaal daags gedoseerde aminoglycosiden onderling heeft vergeleken. Verder toonden we aan dat het oppervlak onder de concentratie-vs-tijd curve (AUC) van de eerste gift de beste voorspeller was van het ontwikkelen van nefrotoxiciteit. Deze resultaten suggereren dat het bijstellen van de dosering en vooral het doseerinterval aminoglycosiden op basis van serum concentraties, afgenomen na de eerste gift, van belang zijn om het risico op nierschade bij individuele patiënten beter in te kunnen schatten.

In hoofdstuk 2.4 hebben we prospectief een nieuw gentamicine doseerregime voor pasgeborenen op een high care unit met (een verdenking op) neonatale sepsis geëvalueerd. Deze studie liet zien dat bij een dosering van $5 \mathrm{mg} / \mathrm{kg}$ elke 36 uur de vooraf vastgestelde 'therapeutische' streefwaarden werden gehaald in meer dan 95\% van de geïncludeer- de patiënten. Een belangrijke toegevoegde waarde van dit onderzoek is de selectie van een populatie pasgeborenen die opgenomen waren op een high care unit. Verreweg de meeste studies op dit terrein zijn uitgevoerd bij pasgeborenen die opgenomen waren op een neonatale intensive care unit (NICU). Zij kennen een veel grotere onderlinge variabiliteit in farmacokinetiek. Daardoor kennen doseerregimes voor een dergelijke populatie pasgeborenen vaak meerdere startdoseringen afhankelijk van het gewicht, de zwangerschapsduur en postnatale leeftijd van de pasgeborene. Wij concluderen dat het onderzochte doseerregime leidt tot een betrouwbare en gelijkmatige gentamicineblootstelling in de onderzochte populatie. Daarnaast suggereren de resultaten dat relatief complexe schema's niet noodzakelijk zijn voor het doelmatig doseren van deze antibiotica bij pasgeborenen die opgenomen zijn op een high care unit. 
Tot slot liet deze studie zien dat de AUC van een gift gentamicine bij meer premature, lichtere pasgeborenen gelijk was aan de AUC bij a terme pasgeborenen met een hoger geboortegewicht.

De afwezigheid van een effect van geboortegewicht en zwangerschapsduur op de AUC kon verklaard worden doordat met een toename van gewicht en zwangerschapsduur de halfwaardetijd korter werd terwijl het verdelingsvolume (L/kg) afnam.

Hoofdstuk 3.1 is een systematische review van de bestaande literatuur waarbij de inter-patiënt variabiliteit van vancomycine serum concentraties tussen continue en intermitterende infusie onderling zijn vergeleken. Deze literatuurstudie liet zien dat in populaties die behandeld werden met continue infusie versus intermitterende infusie de interpatiënt variabiliteit in serum concentratie gemiddeld vijftig procent lager was. Deze interessante bevinding is gebaseerd op 34 gepubliceerde artikelen en gaf aanleiding tot het opzetten van een direct vergelijkend onderzoek tussen intermitterende en continue infusie middels een retrospectieve cohort studie (hoofdstuk 3.2). Ook dit onderzoek liet zien dat de inter-patiënt variabiliteit van vancomycine serumconcentraties ongeveer tweemaal lager was bij de met continue infusie behandelde populatie in vergelijking met de groep patiënten die vancomycine via intermitterende infusie kreeg toegediend. Daarnaast toonde het laatstgenoemde onderzoek aan dat de correlatie tussen plateauconcentraties en de geschatte AUC bij continu doseren sterker was dan de correlatie tussen dalspiegels en geschatte AUC bij intermitterend gedoseerde patiënten. Aan deze resultaten liggen volgens ons twee oorzaken ten grondslag. Om te beginnen heeft de 'timing' van afname van het serummonster in geval van dalspiegels bij intermitterende infusie invloed op de gemeten concentratie. Een effect dat in geval van continu doseren en plateauspiegels vrijwel afwezig is na het bereiken van steady state condities. Verder wordt de inter-patiënt variatie in dalspiegels tijdens intermitterende infusie eveneens beïnvloed door verschillen in distributievolume in tegenstelling tot de variatie in plateauspiegel bij continu doseren. Patiënten met een relatief hoog distributievolume van vancomycine hebben hogere intermitterende doseringen nodig om therapeutische dalspiegels te bereiken, hetgeen zal resulteren in een grote 'delta' tussen piek- en dalspiegel en daarmee in een hoge AUC/ dalspiegel ratio. 
Wederom uitgaande van steady state condities zullen de plateauspiegels van continu gedoseerde patiënten niet beïnvloed worden door de inter-patiënt verschillen in distributievolume van vancomycine (hoofdstukken 3.1 en 3.2).

In hoofdstuk 3.3 wordt een methode gepresenteerd voor het ontwerpen van een vancomycine doseernomogram voor continue infusie waarbij gebruik gemaakt werd van historische data verkregen binnen de onderzochte populatie.

Vervolgens werd het ontworpen doseernomogram geïmplementeerd in de klinische praktijk en prospectief geëvalueerd. Dit hoofdstuk biedt handvatten ten behoeve van clinici die een switch van intermitterend naar continu doseren van vancomycine overwegen. Ondanks dat het niet onze intentie was om een doseernomogram voor de continue infusie van vancomycine te ontwerpen voor gebruik in andere klinisch geinfecteerde ziekenhuispopulaties, komt het algoritme wel overeen met het enige andere prospectief gevalideerde doseernomogram opgesteld door Pea en collega's.

In hoofdstuk 4 worden allereerst de samengevatte resultaten van de hoofdstukken 2 en 3 beschreven. Daarna wordt dieper ingegaan op de klinische implicaties. Het hoofdstuk eindigt met enkele suggesties voor toekomstige studies. Met betrekking tot de behandeling met aminoglycosiden is ons belangrijkste advies tobramycine te verkiezen boven gentamicine bij patiënten voor wie een langere behandelduur geïndiceerd is. Volgens onze resultaten, welke gesteund worden door de resultaten van direct vergelijkend onderzoek uitgevoerd ten tijde van het tijdperk van meerdaags doseren, geeft dit een lagere kans op het ontwikkelen van nefrotoxiciteit. Er lijken nauwelijks argumenten te pleiten tegen dit therapieadvies. Er is bewijs dat tobramycine wereldwijd minder microbiële resistentie kent en tevens dieper penetreert in weefsels dan gentamicine. Daarnaast liggen de huidige inkoopprijzen van intraveneuze tobramycine preparaten lager dan de prijs van de gentamicine equivalenten. Wij stellen dan ook voor om een prospectief gerandomiseerde studie te ontwerpen waarin klinische uitkomstmaten van eenmaal daags gedoseerd gentamicine en tobramycine worden vergeleken. Met betrekking tot de modus van toedienen van vancomycine blijkt uit ons literatuuronderzoek dat er weinig direct vergelijkend onderzoek is uitgevoerd naar de klinische uitkomstmaten van intermitterende en continue infusie. 
Op grond van het momenteel beschikbare bewijs lijkt toediening per continu infuus bij de meeste indicaties niet inferieur aan intermitterende toediening en heeft het mogelijk voordelen bij de behandeling van osteomyelitis en pleuraempyeem. Daarnaast zijn er aanwijzingen dat continue infusie minder nefrotoxisch is dan intermitterende infusie. Tevens is continue infusie van vancomycine bewezen goedkoper en kent het enkele andere praktische voordelen, zoals eenvoudigere monitoring van de blootstelling en de mogelijkheid tot eenvoudiger doorbehandelen in de thuissituatie.

Tot slot zijn er geen grote bezwaren die pleiten tegen het toepassen van continue infusie van vancomycine. Gezien bovenstaande argumenten prefereren wij continue infusie van vancomycine boven intermitterende infusie. Echter, grote prospectieve multicenter gerandomiseerde klinische studies zijn nodig om de verschillen in behandeluitkomsten tussen continue en intermitterende infusie van vancomycine beter te karakteriseren. 



\section{Dankwoord}

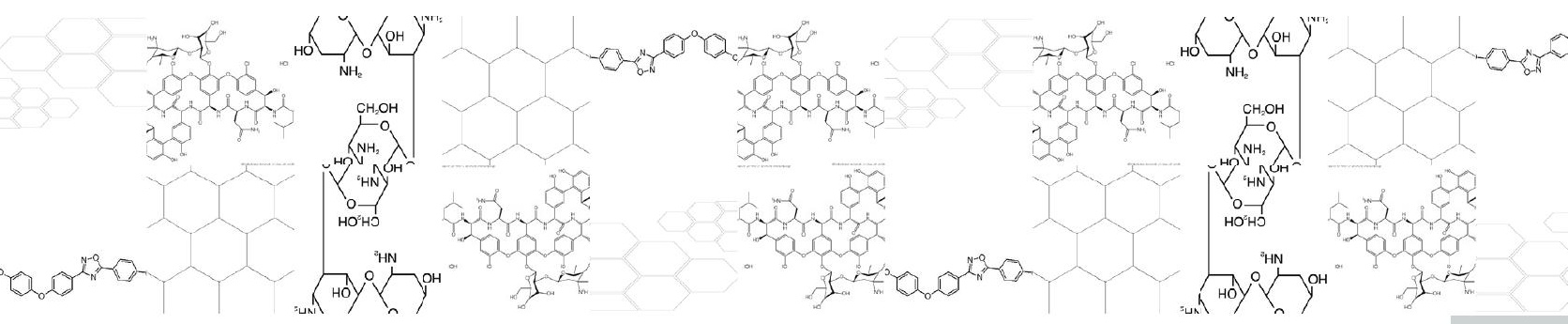


Dit promotieonderzoek is in 2009 begonnen als gevolg van een uit de hand gelopen onderzoeksstage te Den Haag gedurende mijn opleiding tot ziekenhuisapotheker in het Groene Hart Ziekenhuis. Tijdens deze stage ontstond mijn interesse voor klinisch onderzoek. Kort na aanvang van deze "buitenpromotie" aan de Universiteit Maastricht en mijn registratie tot ziekenhuisapotheker startte ik als ziekenhuisapotheker laboratorium te UMC Utrecht. Zo kon het gebeuren dat dit proefschrift gevuld met Goudse en Haagse data te Maastricht verdedigd zal worden door een Utrechtse ziekenhuisapotheker.

Bovenstaande constructie is allesbehalve standaard en voor het slagen ervan ben ik een groot aantal mensen dank verschuldigd. Graag wil ik een ieder bedanken die mij direct of indirect geholpen heeft bij mijn magnum opus. Zonder jullie werk en steun was dit proefschrift niet als dusdanig tot stand gekomen. Een aantal mensen wil ik hieronder in het bijzonder bedanken.

Allereerst mijn 'promotieteam'. Promotoren Kees Neef en Daan Touw en co-promotor Arthur van Zanten. Daan, eigenlijk ben jij degene die mij motiveerde en inspireerde om mijn registratieonderzoek uit te bouwen naar een promotie. Je enthousiasme werkt aanstekelijk op jonge onderzoekers. Ook je toewijding voor klinisch (farmacologisch) onderzoek en voor je promovendi heb ik enorm gewaardeerd de afgelopen jaren. Daarnaast we zaten altijd snel op een lijn; van nulhypothese tot indiening van het manuscript. Heerlijk! Kees, samen met Daan vormde je mijn aanvankelijke 'begeleidings- team'. Een promotor op bijna 200 $\mathrm{km}$ afstand is een uitdaging, maar gelukkig was je altijd goed benaderbaar en flexibel bij het inplannen van een volgend promotieoverleg. Afspreken in de centrale Domstad aansluitend aan de KKGT vergaderingen leek logistiek de beste oplos- sing. Het duurde overigens wel even voordat ik de juiste gangen in het doolhof richting NVKC gebouw wist te vinden. Bedankt voor je begeleiding! Arthur, je sloot je pas later aan bij het team. De timing was niettemin perfect. We hebben elkaar leren kennen op een studiereis naar een infusenfabriek in Baskenland. Het was me snel duidelijk dat je een intensivist was met buitengewone interesse in farmacologie van antibiotica en bevlogen innovator van de gezondheidzorg. Naast je onmisbare 'klinische bril' leerde ik onder andere van je hoe een artikel weg te zetten en hoe zo elegant mogelijk de discussie met referenten aan te gaan. Dank! 
Beste (ziekenhuis)apothekers van het UMC Utrecht, mijn directe collega's, ook jullie steun en interesse heb ik als bijzonder ervaren. Onze open cultuur en bekwame vakgroep leent zich uitermate voor 'even sparren' of desgewenst raad inwinnen bij een ervaren onderzoeker. Met aan zekerheid grenzende waarschijnlijkheid zullen er dit jaar vier apothekers binnen onze groep promoveren. Een onwaarschijnlijke oogst, die niet alleen aan de inspanning van dat fortuinlijke viertal is toe te wijzen maar een verdienste van formaat van de hele vakgroep genoemd mag worden. Getuige het snel groeiende aantal onderzoekers en het gunstige research klimaat zullen er zeker nog vette jaren volgen. Het voert te ver om iedereen van jullie individueel te bedanken, maar tot een aantal van jullie wil graag persoonlijk het woord richten. Toine, werkelijk bijzonder dat je vanaf het moment van mijn indiensttreding mijn externe promotieonderzoek hebt gefaciliteerd. Naast de permissie voor de benodigde tijd stond je me altijd met raad en daad terzijde. Niet alleen op gebied van mijn promotieonderzoek maar eigenlijk ziekenhuisfarmaciebreed. Samen we hebben de afgelopen jaren veel bereikt en werken in jouw apotheek geeft mij nog dagelijks plezier. Ik hoop dit voort te kunnen zetten en daar waar mogelijk uit te bouwen. Karin, ons hoofd Triple O, je passie en je gedrevenheid voor onderzoek werken aanstekelijk. Age quod agis! Barbara, jou wil ik heel graag bedanken voor de fantastische tijd en gezelligheid in het UMCU. Ik heb aan jou veel steun gehad als medepromovendus, net als aan Esther trouwens - gedeelde smart is halve smart. Je bent een geweldige collega en je positieve energie zal gemist worden in 't Utrechtse met je vertrek naar 't schone Brabantse land. Bedankt en veel succes in Tilburg!

Marcel en Tessa, kamergenoten, wat een lief en leed hebben we met elkaar gedeeld de afgelopen jaren. Marcel, mijn partner in PK crime, iedere farmacologische discussie met jou is een genot - wie er uiteindelijk ook gelijk heeft of gelijk neemt! Je bent de collega die niet alleen in den lijve maar ook in farmaceutische gedachten het dichtst bij mij staat. Tessa, jou wil ik ten eerste heel graag bedanken voor mijn huidige prachtige baan. Bij mijn sollicitatiegesprek dacht ik werkelijk in het ootje genomen te worden, toen je zei dat je zelf de QC wilde gaan doen en tegelijk de TDM aan mij over te laten. Bedankt ook dat ik al jouw mooie ideeën voor onderzoeken uit heb mogen voeren In een vergelijkbaar opgezet onderzoek bij kinderen met taaislijmziekte kwamen zij tot dezelfde conclusies. 
- zeker de helft van mijn huidige wetenschappelijke output bevat een vleugje van jouw gedachtengoed. Met uitzondering van onze Kruyt periode delen we in onze kamer al 5 jaar onze 'highs and lows'. Misschien kunnen we inmiddels een Brabants tegeltje overwegen: 'Ge mot 't nie opkroppen, verrekte m.....' of houd je het toch liever bij 'GMP is our lifestyle'? Bedankt ook dat je me hebt geleerd 'hoe de hazen lopen' in een academische tent.

Graag wil ik ook alle analisten van het laboratorium van de UMCU apotheek danken. Vincent, Evelien, Sabine, Klaas, Annelies, Mohsin, Mandy, Kamiel, Yvette, Bea, Anneke en Janneke. Ik kan nog dagelijks genieten van het opzetten van innovatieve analyses en ben daarnaast trots op hoe jullie jezelf ontwikkeld hebben. Werken met jullie op ons lab blijft interessant. Bedankt voor jullie steun en interesse. Ook de analisten van de apotheeklaboratoria van het Groene Hart Ziekenhuis - Jan Leen, Ellen en Kees en alle analisten van de Apotheek Haagse Ziekenhuizen wil ik bedanken voor hun hulp bij het uitvoeren van de analyses en het verzamelen van de data gebruikt voor dit promotieonderzoek.

Verder wil ik mijn voormalige Goudse collega's bedanken. Aan mijn opleidingstijd in Gouda bewaar ik warme herinneringen en nog steeds goede contacten. Tot een aantal mensen wil ik een persoonlijk woord van dank richten. Allereerst Monique, ik ben je zeer erkentelijk voor de kans om mijn registratieonderzoek in Den Haag onder wetenschappelijke begeleiding van Daan uit te voeren. Natuurlijk hield je wel de planning strak in de gaten. Daarnaast heb ik enorm veel van je geleerd over het ziekenhuisapothekerschap. Deze laatste woorden gelden tevens voor Mignon, opleider te Gouda tijdens het staartje van mijn registratiefase. Sardha, Annemien, Charlotte, Hans, Katja, jullie hebben mij mede gevormd tot de ziekenhuisapotheker en onderzoeker die ik nu ben. Ik ben jullie dankbaar voor het overdragen van jullie ervaring en kennis en ook voor alle gezelligheid natuurlijk. Tot slot wil ik graag Franny en Anke bedanken, want jullie zijn allebei heel erg belangrijk voor mij geweest op zowel professionele als persoonlijke vlak.

Nog een woord van dank voor alle studentstagiairs die dit onderzoeksproject versleten heeft. Twee van jullie zijn voor mij van onschatbare waarde geweest. Walaa, wat heb jij hard gewerkt tijdens je stage. Alles begint met inzet en toewijding. Aan beide was bij jou geen gebrek. En 
Suzan, als er zoiets als een ideale stagiair bestaat dan kom jij daar aardig bij in de buurt. Veel succes met afstuderen en in je aanstaande carrière. Bedankt ook alle verpleegkundigen en arts-assistenten - in het bijzonder Renske - op de afdeling longgeneeskunde van het HAGA ziekenhuis voor de inclusie van de CF patiënten voor de CIRCA studie. Dank ook aan alle co-auteurs voor jullie hulp bij het opstellen van METC aanvragen, bij schrijven van de manuscripten en voor het delen van jullie netwerken.

Natuurlijk wil ik mijn familie graag bedanken - om te beginnen Els, mijn moeder en Gerard, mijn vader en John. Jullie hebben me altijd aangemoedigd - maar nooit gepusht - om mijn talenten te ontwikkelen. Dit boekje is ook jullie verdienste. Helaas zal mijn vader mijn 'hora est' niet mee kunnen maken, maar ik weet zeker dat hij trots op me zou zijn geweest. Ook een vermelding waardig zijn de grootste oppasactiviteiten van mijn moeder en John. Sam, Liv en Ties hebben bij jullie hun tweede thuis! Mijn bro's Jelle en Thomas, jullie zorgden vroeger voor de nodige 'serious competition' - ik herinner me onder andere rondvliegende tafeltennisbatjes. Juist voor die potjes tafeltennis wil ik jullie bedanken. De 'innerlijke drive', die nodig is om een proefschrift af te ronden, vindt vermoedelijk hierin zijn oorsprong. Ik ben gelukkig met twee fantastische broers en hoop dat we nog veel lol met elkaar kunnen hebben. Een bedankje aan mijn vrienden is op zijn plaats en in het bijzonder Bram en Erik, mijn vrienden voor het leven, mijn mede-brave huisvaders. Na ruim 16 jaar vriendschap kennen jullie mij van binnen - tot op orgaanniveau - en van buiten. Door promoties, werk en vooral het toegeven aan onze voortplantingsdrang - zonder oordeel een drang bij de een iets groter dan bij de ander - zien we elkaar wat minder in deze tropenjaren. Echter, onze kroost zal eens het nest verlaten en oude tijden zullen herleven! Dank voor jullie steun. Erik, bedankt ook dat je me bij zal staan als paranimf. Ferdi, mijn tweede paranimf, in Gouda waren we ooit 'concullega's', een gezonde competitie die louter winnaars en een vriendschap heeft opgeleverd. Ik vond het een eer dat ik tijdens jouw verdediging je paranimf mocht zijn en dat jij nu mijn paranimf bent.

Tot slot wil ik mijn Marieke bedanken en uiteraard niet alleen voor de opmaak van het binnenwerk en het ontwerp van de omslag. We zijn al tien jaar samen en hebben in die tijd al veel meegemaakt. Je bent mijn 
grote liefde en mijn beste maatje. Dat dit boekje het levenslicht heeft mogen aanschouwen, is zonder enige twijfel ook de verdienste van mijn veelzijdige en multitaskende verloofde. De geboorte van dit boekje, mijn 'vierde kindje', is tot stand gekomen met geregeld hevig gepuf en gesteun van mijn kant. Gelukkig was jij er altijd om me te steunen, net als ik er omgekeerd voor jou was tijdens de geboortes van onze kinderen - althans in mijn herinneringen is dat zo. Lieve Marieke, we gaan samen verder met het verwezenlijken van onze dromen. Ik ben trots op je en hou van je. 



\section{List of publications}

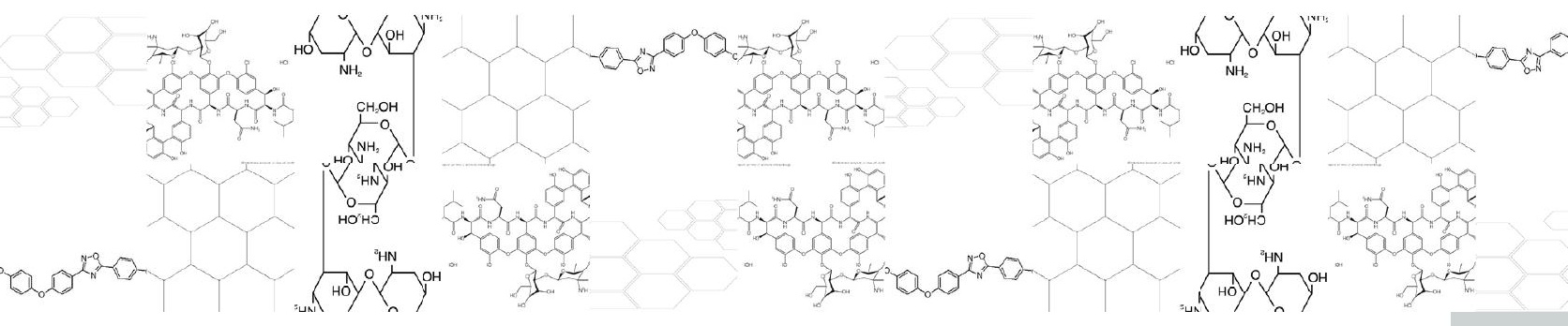


1. Design and prospective validation of a dosing instrument for continuous infusion of vancomycin: a within-population approach. van Maarseveen EM, Bouma A, Touw DJ, Neef C, van Zanten AR. Eur J Clin Pharmacol. 2014 Aug 30.

2. Bioavailability of cyclophosphamide and vincristine after intraperitoneal administration in cats. Voorhorst MJ, van Maarseveen EM, van Lankveld AJ, Teske E. Anticancer Drugs. 2014 Aug 20.

3. Managing drug-drug interactions in an HIV-infected patiënt receiving antiretrovirals, anti-HCV therapy and carbamazepine: a 'tour de force' for clinical pharmacologists. Burger DM, Arends JE, Jacobs BS, van Elst-Laurijsen DH, de Kanter CT, van Maarseveen EM, Verwey-van Wissen CP, Hoepelman Al. Int J Antimicrob Agents. 2014 Jul;44(1):868.

4. Once-daily dosed gentamicin is more nephrotoxic than once-daily dosed tobramycin in clinically infected patients. van Maarseveen E, van Buul-Gast MC, Abdoellakhan R, Gelinck L, Neef C, Touw D. J Antimicrob Chemother. 2014 Sep;69(9):2581-3.

5. Fludarabine and exposure-targeted busulfan compares favorably with busulfan/cyclophosphamide-based regimens in pediatric hematopoietic cell transplantation: maintaining efficacy with less toxicity. Bartelink IH, van Reij EM, Gerhardt CE, van Maarseveen EM, de Wildt A, Versluys B, Lindemans CA, Bierings MB, Boelens JJ. Biol Blood Marrow Transplant. 2014 Mar;20(3):345-53.

6. The Quantitative Effect of Serum Albumin, Serum Urea, and Valproic Acid on Unbound Phenytoin Concentrations in Children. Heine RT, van Maarseveen EM, van der Westerlaken MM, Braun KP, Koudijs SM, Berg MJ, Malingré MM. J Child Neurol. 2013 May 13;29(6):803810.

7. Pretransplantation pharmacokinetic assessments to predict posttransplantation dosing requirements in renal transplant recipients: what is known? van Maarseveen E, van Zuilen AD. Clin Pharmacol Ther. 2013 Apr;93(4):306-7. 
8. Pretransplantation pharmacokinetic curves of tacrolimus in HIV-infected patients on ritonavir-containing CART: a pilot study. van Maarseveen EM, Crommelin HA, Mudrikova T, van den Broek MP, van Zuilen AD. Transplantation. 2013 Jan 27;95(2):397-402.

9. Drug-drug interactions between antiretroviral and immunosuppressive agents in HIV-infected patients after solid organ transplantation: a review. van Maarseveen EM, Rogers CC, Trofe-Clark J, van Zuilen AD, Mudrikova T. AIDS Patiënt Care STDS. 2012 Oct;26(10):568-81.

10. Activated charcoal for GHB intoxication: an in vitro study. Neijzen R, van Ardenne P, Sikma M, Egas A, Ververs T, van Maarseveen E. Eur J Pharm Sci. 2012 Dec 18;47(5):801-3.

11. Pharmacokinetics of paroxetine, a selective serotonin reuptake inhibitor, in Grey parrots (Psittacus erithacus erithacus): influence of pharmaceutical formulation and length of dosing. van Zeeland YR, Schoemaker NJ, Haritova A, Smit JW, van Maarseveen EM, Lumeij JT, Fink-Gremmels J. J Vet Pharmacol Ther. 2013 Feb;36(1):51-8.

12. [Continuous and intermittent infusion of vancomycin equally effective: review of the literature]. van Maarseveen EM, Man WH, Touw DJ, Bouma AW, van Zanten AR. Ned Tijdschr Geneeskd. 2011;155(42):A2667.

13. Outcomes of kidney transplantation in HIV-infected recipients. van Maarseveen EM, van Zuilen AD, Mudrikova T. N Engl J Med. 2011 Feb 17;364(7):683; author reply 684 .

14. Preparation and characterization of folate-targeted $p E G$-coated pDMAEMA-based polyplexes. van Steenis JH, van Maarseveen EM, Verbaan FJ, Verrijk R, Crommelin DJ, Storm G, Hennink WE. J Control Release. 2003 Feb 21;87(1-3):167-76. 



\section{Valorization paragraph}

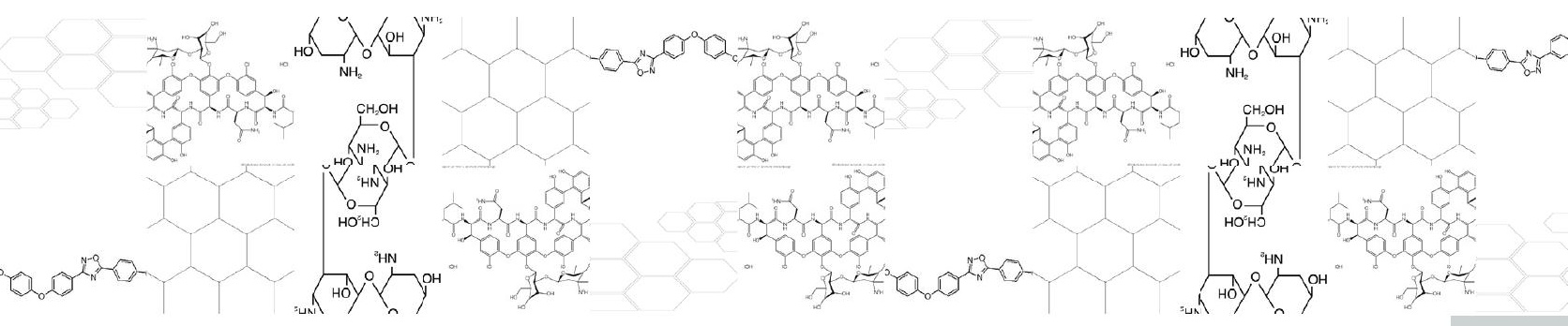


As the current drug development pipeline wanes new classes of antibiotics, it is essential we learn to exploit our current antibiotic arsenal optimally by 'rediscovering' 'older' antimicrobial agents. Aminoglycosides, e.g. gentamicin and tobramycin and glycopeptides, e.g. vancomycin are considered antibiotics of older classes as they were marketed in the 1950s. Aminoglycosides and vancomycin are cornerstones of the current antibiotic armamentarium because of their activity against multidrug-resistant pathogens that can cause high rates of mortality and morbidity especially in hospitals. However, tobramycin and gentamicin have narrow therapeutic ranges and can induce both serious damage to the kidneys and ears limiting its clinical use. Clinicians prescribing vancomycin and other glycopeptides, on the other hand, are faced with emerging resistance among methicillin-resistant Staphylococcus aureus (MRSA) and vancomycin-intermediate S. aureus (VISA) strains worldwide. Although vancomycin still is the antibiotic of choice for these types of infections, the development of drug resistance is increasing. Futhermore, there is a risk of treatment failure if minimal drug exposure targets are not reached and maintained in individual patients. Vancomycin resistance is a serious threat that needs to be dealt with in the years to come if we want to preserve this vital antibiotic for future generations. Therefore, we explored pharmacologically-based strategies to optimize aminoglycoside and vancomycin therapies.

In this thesis data are presented on gentamicin, tobramycin and vancomycin pharmacokinetics and clinical outcomes in clinically infected populations. The patients populations investigated include cystic fibrosis patients, neonates, general ward and critically ill patients. Patiënt populations that show aberrant aminoglycoside and vancomycin pharmacokinetics and that are potentially susceptible to toxicity and treatment failure. Moreover, therapeutic drug monitoring (TDM) is recommended to optimize aminoglycoside and vancomycin therapy in individual patients.

In chapters 2.1 and 2.2 we showed that the time period of administration did not affect the clearance or exposure in terms of area-under-thecurve (AUC) in CF patients, general ward patients and ICU patients. Hence, in daily clinical practice 24-hour intervals should be maintained unless therapeutic drug monitoring results indicate that the dosing interval needs to be extended. Shifting administration times to the morning period - which is not uncommon in current practice - is not 
likely to offer a benefit to these patients. It can, however cause side effects or treatment failure due to shortening or extending administration intervals, respectively. Although our findings deserve to be confirmed, results from Chapter 2.3 showed a higher nephrotoxic potential of once daily dosed (ODD) gentamicin over ODD tobramycin in clinically infected patients treated for at least 3 days which may lead to the revision of (inter)national and local treatment protocols. In patients who are at high risk of developing nephrotoxicity and/or are likely to be treated over a longer period, tobramycin should be preferred over gentamicin. In fact, European cystic fibrosis treatment guidelines already advocate choosing tobramycin over gentamicin for this reason. The same concerns with regard to cumulative toxicity may apply to other highly exposed populations, e.g. patients treated for endocarditis. In order to optimize treatment with aminoglycosides 'early' AUC monitoring in addition to 'trough levels' should be implemented in daily routine, since our data suggest the AUC of the first administration is a strong predictor of nephrotoxicity.

The added value to literature of our study results presented in Chapter 2.4 on gentamicin dosing in neonates lies in the specific neonatal population investigated. Albeit the results deserve to be prospectively confirmed in a second independent cohort of neonates admitted to a level II special care nursery, our findings show that a more uniform dosing protocol of $5 \mathrm{mg} / \mathrm{kg}$ per 36 hours can be implemented in level 2 unit compared to more complex dosing protocols proposed in neonates admitted to a level III intensive care unit. This offers the potential use of an easy one-dose-fits-all protocol, which is likely to reduce prescribing errors in neonates. The robustness of the investigated dosing regimen may also reduce the need for TDM and thereby the number of gentamicin blood samples. Reduction of blood sampling in neonates lowers the risk of infections and is one of focus points of the World Health Organization (WHO).

As described in Chapter 3.1 vancomycin continuous dosing protocols are rapidly gaining interest and are being implemented in clinical practice. Continuous infusion of vancomycin might also be preferred since the drug preparation time, the staff workload and costs are reduced and may also shorten hospital length of stay. Apart from the practical advantages over intermittent infusion, continuous infusion of vancomycin results a more predictable exposure and thus has better target attainment odds as shown in Chapter 3.1 and 3.2 are better. 
Finally, based on our methodology described in chapter 3.2 a 'tailored' dosing algorithm of vancomycin can be designed for clinically infected populations aiming for the desired target exposure.

The results reported in this study are of considerable interest to clinical pharmacologists and clinicians especially infectious disease specialists, clinical microbiologists, pediatricians and intensive care physicians.

Furthermore, our data can contribute to future antibiotic (inter)national guidelines. Moreover, the cost savings achieved by higher cure rates, lower percentages of patients experiencing treatment related side effects and higher efficiency are of great interest to policymakers. The increase in drug resistance urges both the private and public sector to keep these older antibiotics available. In our opinion consortia should be formed to investigate potential applications and new indications of these antibiotics by designing innovative drug formulations and performing clinical studies. For instance, the European Union is currently running a program promoting private and public collaboration to test new formulations of inhaled antibiotics in patients with cystic fibrosis. Finally, the suggestions for future studies made in chapter 4 are of interest to other researches in the field of antibiotics. Studies call for more studies. To conclude, in an era of increasing antibiotic resistance against our current first line of defense, this thesis provides pharmacology-based strategies striving for a safer and more effective clinical usage of tobramycin, gentamicin and vancomycin. 



\section{Curriculum Vitae}

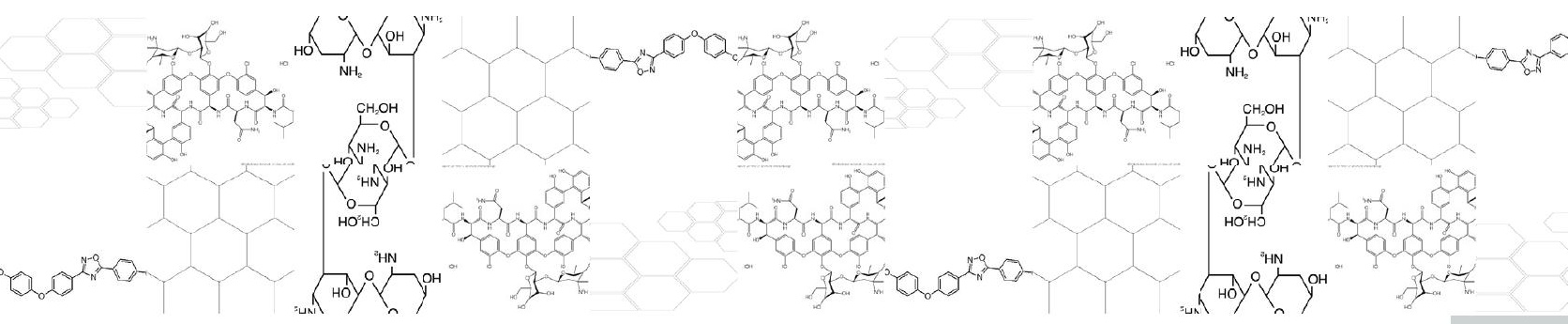


Erik van Maarseveen was born on the 17th of May 1979 in Tiel. He graduated at the Gymnasium of the Tiel College in 1998.

He studied Pharmaceutical Sciences from 1998 till 2004 at the University of Utrecht and received his Pharmacist Degree in 2005. That year he also completed all theoretical courses of the Master program "Business and Economics for Beta's" at the University of Utrecht.

In 2005 he was employed as a pharmacist to work on a project promoting an early switch of intravenous to oral antibiotics at the Groene Hart Ziekenhuis Gouda. In 2006 he started his training as a hospital pharmacist at the Groene Hart Ziekenhuis. He has been working as a registered hospital pharmacist since 2010 at the University Medical Center Utrecht, where he is the lead pharmacist of the therapeutic drug monitoring and toxicology laboratory. In 2015 he will start his training as a clinical pharmacologist at the University Medical Center Utrecht.

In 2009 he started his $\mathrm{PhD}$ on the pharmacology of aminoglycosides and vancomycin at Maastricht University, which resulted in this thesis. Next to antibiotics

his current research focusses on the pharmacology of immunosuppressive, antiretroviral and anti-cancer drugs.

Erik van Maarseveen is engaged to Marieke Velthuis. They are proud to be the parents of three beautiful children; one daughter Liv (2010) and two sons Sam (2008) and Ties (2012). 